UC. $90 \mathrm{i}$

Issued: April 1987

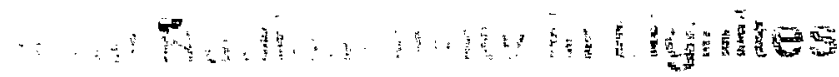 6
}

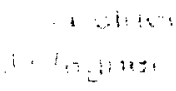

\section{DISCLAIMER}

This report was prepared as an account of work sponsored by an agency of the United States Government. Neither the United States Government nor any agency thereor, nor any of their employees, makes any warranty, express or implied, or assumes any legal liability or responsivility for the accuracy, completeness, or usefulness of any information, apparatus, product, or process disclosed, or represents that its use would not infringe privately owned rights. Reference herein to any specific commercial product, process, or service by trade name, trademark, manufacturer, or otherwise does not necessarily constitute or imply its endorsement, recommendation, or favoring by the United States Government or any agency thereof. The views and opinions of authors expressed herein do not necessarily state or reflect those of the United States Government or any agency thereof.

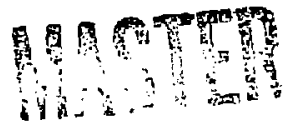




\title{
NATURAL RADIOACTIVITY IN LIGNITES AND LIGNITE ASH: FINAL REPORT
}

by

N. Roy Greiner and Paul Wagner
$L A--10942-M S$

DE87 007720

\begin{abstract}
Natural radioactivity in a Texas lignite field and in leachates from lignite ash from power plants was measured. The radioactivity concentrations found (4 ppm uranium in the lignite, $16 \mathrm{ppm}$ uranium in the lignite ash, and a few picocuries of natural radionuclides per liter in the leachates) do not differ appreciably from those found previously in similar samples and in natural inaterials such as soils and ground waters. Additional information on stable elements are reported for both the lignites and the leachates.
\end{abstract}

\section{SUMMARY}

This study provides new data on the natural radioactivity content of solid wastes and their leachates that result from burning lignite for power generation. The natural radioactivity in various coals and their associated waste products has been studied extersively, but notably lacking are data on lignites from geological formations containing high concentrations of uranium and data on the leachates of ash from power plants burning lignites. This study addresses both areas and extends the data base in each case.

Samples were taken from the major searns of a lignite field in Texas that is in a geological formation containing large concentrations of uranium. The sample boreholes were distributed evenly over the field to assure representation of the whole field. Samples of the geological material in direct contact with the lignite were also included in this study. Most of the radioactivity was found in the material in contact with the lignite rather than in the lignite itself. The lignite contains about twice the radioactivity of the average US coa!; but, because these lignites also contain about twice the ash content of the average US coal, the ash contains about the same radioactivity as the average US coal ash. The uranium content of these lignites is about the same as that of typical soils.

Ash from two power plants that burn lignites was leached over 2 weeks. Samples of the leachate, which were taken daily, were analyzed for both radionuclides and stable 
elements. Because minimal quantities of radionuclides were found, we conclude that the radionuclide content of these leachates poses no significant environmental threat.

When these results are combined with other data on radioactivity in coals and associated waste products, our results in this study are not notably different from those of previous studies and none of the materials appear to pose any significant environmental threat from radioactivity.

\section{INTRODUCTION}

Before this study, the data base contained information on the radionuclide content of several coals, ashes, and leachates. ${ }^{1,2}$ However, no data were available for lignites associated with uranium-bearing geological formations or for the leaching of lignite ashes. This study assesses the radionuclide content of a mineable lignite field in a geological formation (the Yegua-Jackson) that contains mineable uranium at another location. We aiso report results of leaching experiments for ashes from two power plants that burn lignites. The radionuclide content of ashes can be estimated with confidence if representative samples of the raw lignite are available for analysis, but the leachability of radionuclides from the ash depends critically on the physical and chemical character of the ash. This character is determined by the detailed chemical history of the minerals in the lignite as they go through the combustion process. Because this history is very difficult, if not impossible, to describe or to duplicate adequately in the laboratory, producing an ash truly representative of the ash from a given power plant is also very difficult. For this reason, we chose to do leaching experiments on composite ash samples representative of a day's run of two operating power plants.

Our goal was to determine the radionuclide content in the solid wastes and leachates generated by using lignites as fuels for electric power generation. This study shows the distribution of radionuclides in a whole lignite field and in the geological material in contact with the lignite. Because of the variability of radionuclide content in individual samples, it is necessary to analyze many samples to obtain a good estimate of the radionuclide content of the field. To this end, several samples distributed over the field were taken from each major lignite seam and from the material in contact with the seams.

The power plant ash samples were studied to determine the leachability of radionuclides and the effect of the nonradioactive components on the leachability of the radionuclides. These combined results show that the properties of the materials studied fall within the range of properties for similar materials studied previously. Furthermore, these materials show no unusually large amounts of radioactivity nor unusual leachability. 


\section{ELEMENTAL ANALYSES OF LIGNITE SAMPLES}

Seventy-six samples of the lignites and associated geological material were analyzed by neutron activation analysis (see Appendix). The results were subjected to a variety of multivariate statistical analyzes to ascertain groupings of the samples (Table I). This analysis divided the samples into two groups: one containing mainly lignites (clusters 1, 3, 4, 5, 7, 9, and 10 ) and the other containing mainly tuffs (clusters 2, 6, and 8 ). The lignites averaged about $4 \mathrm{ppm}$ uranium and $12 \mathrm{ppm}$ thorium, whereas the tuffs averaged about $11 \mathrm{ppm}$ uranium and $35 \mathrm{ppm}$ thorium.. Additional examination of the data by cluster analysis revealed more details of the sample set, the most significant being that the lignite samples could be divided into three subsets with different calcium:iron ratios. The presence of calcium tends to make leachates basic, whereas iron tends to make them acid. One subset of lignites was higher than average in calcium (clusters 3, 7, and 10), another was higher than average in iron (clusters 4 and 9), and the third was average in both calcium and iron (clusters 1 and 5). Many potentially toxic metals have low solubility in basic solutions with the pH controlled by $\mathrm{Ca}(\mathrm{OH})_{2}$ and $\mathrm{CaCO}_{3}$, two common calcium minerals found in lignites and lignite ash. In solutions where the $\mathrm{pH}$ is controlled by iron, many of these metals are soluble enough to attain toxic concentrations. Precise predictions about $\mathrm{pH}$ and metal solubility in the ash leachate require a complete description of the mineral content and the combustion chemistry of the mineral matter, details that were beyond the scope of this study. In view of the present limited knowledge about the combustion chemistry of coal minerals in power plants, environmental estimates are best based on experimental data from actual power plant ash.

The significance of these results to a study of radionuclides is the higher than average amounts of uranium and thorium in the lignites, since these elements are the origin of most radioactivity in these samples. Some radioactivity is associated with potassium, but this source is ubiquitous in all natural materials ${ }^{3}$ and its presence in these samples is insignificant with regard to radiation dose to humans. The significance of the uranium and thorium decay chains has been discussed extensively in the literature 4 and was reviewed in a previous report related to this project. ${ }^{1}$

We will at this point eliminate thorium from further discussion of radiologic dose because thorium itself is immobile in the environment because of the insolubility of its compounds in natural media. Furthermore, its radioactive decay products are short-lived and cannot exist for long in the absence of thorium.

The amounts of uranium in the lignites are larger than the median (1.0 ppm) and average $(1.8 \mathrm{ppm})$ found in US coal samples taken as a whole ${ }^{2}$ (see Fig. 1). Our mean of 4 ppm for the Texas lignite field as a whole is comparable with the thickness-weighted seam 
TABLE I. Cluster Analyses of Neutron Activation Data for Texas Lignite Samples (ppm by weight)

Cluster Means

\begin{tabular}{|c|c|c|c|c|c|c|}
\hline Element & 1 & 2 & 3 & 4 & 5 & 6 \\
\hline $\mathrm{Na}$ & $5 i 15.00$ & 13700.00 & 2000.00 & 5402.50 & 2453.08 & 8251.25 \\
\hline $\mathrm{U}$ & 4.71 & 15.35 & 3.40 & 4.00 & 3.39 & 8.95 \\
\hline Co & 1.72 & 2.46 & 4.68 & 9.77 & 4.22 & 1.09 \\
\hline $\mathrm{Ta}$ & 0.96 & 4.63 & 0.84 & 1.23 & 1.00 & 2.78 \\
\hline $\mathrm{Mg}$ & 1700.00 & 3505.00 & 2475.00 & 4530.00 & 2986.54 & 3797.50 \\
\hline As & 6.30 & 19.05 & 4.43 & 15.23 & 5.21 & 3.37 \\
\hline $2 \pi$ & 95.55 & 177.00 & 55.65 & 84.53 & 55.78 & 95.60 \\
\hline Th & 13.10 & 54.95 & 11.30 & 14.48 & 12.39 & 33.16 \\
\hline $\mathrm{Al}$ & 33800.00 & 88800.00 & 36400.00 & 61850.00 & 46496.15 & 95987.50 \\
\hline $\mathrm{Br}$ & 2.66 & 1.83 & 5.47 & 1.88 & 4.25 & 1.90 \\
\hline $\mathrm{Se}$ & 2.37 & 1.50 & 17.45 & 3.67 & 3.26 & 2.27 \\
\hline $\mathrm{Cl}$ & 122.50 & 670.00 & 70.40 & 74.58 & 87.52 & 77.06 \\
\hline $\mathrm{Sb}$ & 1.50 & 2.22 & 0.89 & 1.29 & 0.85 & 1.07 \\
\hline $\mathrm{Rb}$ & 23.25 & 268.00 & 20.85 & 73.18 & 35.22 & 68.11 \\
\hline $\mathrm{K}$ & 2995.00 & 31100.00 & 3105.00 & 13375.00 & 4783.08 & 11903.75 \\
\hline La & 22.05 & 32.00 & 24.40 & 54.73 & 29.52 & 44.38 \\
\hline Cs & 1.28 & 10.45 & 2.85 & 6.44 & 3.99 & 3.06 \\
\hline $\mathrm{Ca}$ & 10590.00 & 7600.00 & 15200.00 & 7110.00 & 12706.54 & 10435.00 \\
\hline $\mathrm{Sm}$ & 3.42 & 11.95 & 4.21 & 8.40 & 4.50 & 6.77 \\
\hline $\mathrm{Ba}(2)^{\mathrm{a}}$ & 516.50 & 160.50 & 183.00 & 465.50 & 300.05 & 1121.63 \\
\hline $\mathrm{Ti}$ & 1370.00 & 691.50 & 1680.00 & 3130.00 & 2004.62 & 2307.50 \\
\hline $\mathrm{Yb}$ & 3.18 & 7.65 & 4.66 & 4.71 & 3.81 & 3.09 \\
\hline $\mathrm{Ce}$ & 49.45 & 80.55 & 50.30 & 115.00 & 61.60 & 95.51 \\
\hline$v$ & 23.80 & 9.95 & 38.05 & 50.08 & 40.83 & 15.67 \\
\hline Lu & 0.58 & 1.09 & 0.80 & 0.76 & 0.61 & 0.46 \\
\hline Eu & 0.44 & 0.24 & 0.56 & 1.52 & 0.73 & 0.79 \\
\hline $\mathrm{Mn}$ & 235.50 & 432.50 & 393.50 & 241.25 & 399.65 & 236.25 \\
\hline $\mathrm{W}$ & 11.90 & 2.67 & 1.64 & 2.04 & 2.75 & 2.67 \\
\hline $\mathrm{Tb}$ & 0.83 & 1.79 & 0.89 & 1.35 & 0.75 & 0.87 \\
\hline In & 0.19 & 0.29 & 0.16 & 0.17 & 0.15 & 0.18 \\
\hline Sc & 4.91 & 2.42 & 6.26 & 7.37 & 6.67 & 5.07 \\
\hline $\mathrm{Yb}(2)^{\mathrm{a}}$ & 4.04 & 8.60 & 4.92 & 5.10 & 4.28 & 3.70 \\
\hline $\mathrm{Ba}$ & 485.50 & 153.50 & 165.75 & 446.75 & 234.53 & 1128.00 \\
\hline $\mathrm{Cr}$ & 8.70 & 4.82 & 15.60 & 31.70 & 18.70 & 6.59 \\
\hline $\operatorname{Lu}(2)^{\mathrm{a}}$ & 0.63 & 1.12 & 0.78 & 0.75 & 0.64 & 0.47 \\
\hline Dy & 3.83 & 11.40 & 4.99 & 7.84 & 5.01 & 5.58 \\
\hline $\mathrm{Fe}$ & 8055.00 & 10700.00 & 8735.00 & 27125.00 & 13108.46 & 9626.25 \\
\hline Hf & 4.30 & 14.05 & 2.91 & 8.69 & 4.12 & 8.32 \\
\hline
\end{tabular}


Cluster Means

\begin{tabular}{|c|c|c|c|c|c|}
\hline Element & 7 & 8 & 9 & A & Grand Mean \\
\hline $\begin{array}{l}\mathrm{Na} \\
\mathrm{U} \\
\mathrm{Co} \\
\mathrm{Ta}\end{array}$ & $\begin{array}{r}1972.38 \\
3.59 \\
2.25 \\
0.97\end{array}$ & $\begin{array}{r}8711.11 \\
11.02 \\
1.41 \\
2.92\end{array}$ & $\begin{array}{r}3839.44 \\
4.44 \\
6.98 \\
1.58\end{array}$ & $\begin{array}{r}2456.11 \\
3.26 \\
2.08 \\
0.83\end{array}$ & $\begin{array}{r}3816.96 \\
4.93 \\
3.13 \\
1.37\end{array}$ \\
\hline $\begin{array}{l}\mathrm{Mg} \\
\mathrm{As} \\
\mathrm{Zn} \\
\mathrm{Th}\end{array}$ & $\begin{array}{r}2352.86 \\
4.35 \\
43.34 \\
11.26\end{array}$ & $\begin{array}{r}5591.67 \\
6.10 \\
93.83 \\
38.31\end{array}$ & $\begin{array}{r}5921.67 \\
3.20 \\
138.83 \\
16.53\end{array}$ & $\begin{array}{r}2004.36 \\
4.51 \\
38.29 \\
10.22\end{array}$ & $\begin{array}{r}3258.97 \\
5.06 \\
66.75 \\
16.60\end{array}$ \\
\hline $\begin{array}{l}\mathrm{Al} \\
\mathrm{Br} \\
\mathrm{Se} \\
\mathrm{Cl}\end{array}$ & $\begin{array}{r}33171.43 \\
5.08 \\
3.06 \\
197.84\end{array}$ & $\begin{array}{r}98322.22 \\
1.80 \\
2.79 \\
386.25\end{array}$ & $\begin{array}{r}85999.99 \\
1.66 \\
3.77 \\
98.01\end{array}$ & $\begin{array}{r}30984.91 \\
4.81 \\
2.77 \\
83.59\end{array}$ & $\begin{array}{r}52953.66 \\
3.76 \\
3.17 \\
143.49\end{array}$ \\
\hline $\begin{array}{l}\mathrm{Sb} \\
\mathrm{Rb} \\
\mathrm{K} \\
\mathrm{La}\end{array}$ & $\begin{array}{r}0.77 \\
20.07 \\
4172.86 \\
16.27\end{array}$ & $\begin{array}{r}1.43 \\
173.03 \\
19938.89 \\
37.56\end{array}$ & $\begin{array}{r}1.03 \\
81.18 \\
10211.11 \\
39.63\end{array}$ & $\begin{array}{r}0.79 \\
22.18 \\
4006.18 \\
16.09\end{array}$ & $\begin{array}{r}0.95 \\
55.09 \\
7680.51 \\
26.37\end{array}$ \\
\hline $\begin{array}{l}\mathrm{Cs} \\
\mathrm{Ca} \\
\mathrm{Sm} \\
\mathrm{Ba}(2)^{\mathrm{a}}\end{array}$ & $\begin{array}{r}1.85 \\
14152.86 \\
2.71 \\
215.00\end{array}$ & $\begin{array}{r}6.24 \\
7953.89 \\
5.40 \\
279.93\end{array}$ & $\begin{array}{r}9.70 \\
7898.89 \\
5.26 \\
397.83\end{array}$ & $\begin{array}{r}1.88 \\
13928.91 \\
2.59 \\
260.87\end{array}$ & $\begin{array}{r}3.93 \\
11908.46 \\
4.07 \\
329.61\end{array}$ \\
\hline $\begin{array}{l}\mathrm{Ti} \\
\mathrm{Yb} \\
\mathrm{Ce} \\
\mathrm{V}\end{array}$ & $\begin{array}{r}1685.67 \\
2.61 \\
33.29 \\
33.67\end{array}$ & $\begin{array}{r}1402.50 \\
3.07 \\
82.23 \\
12.80\end{array}$ & $\begin{array}{r}3483.89 \\
3.06 \\
80.80 \\
66.41\end{array}$ & $\begin{array}{r}1512.16 \\
2.28 \\
33.54 \\
26.22\end{array}$ & $\begin{array}{r}1904.53 \\
2.98 \\
55.54 \\
32.73\end{array}$ \\
\hline $\begin{array}{l}\mathrm{Lu} \\
\mathrm{Eu} \\
\mathrm{Mn} \\
\mathrm{W}\end{array}$ & $\begin{array}{r}0.45 \\
0.43 \\
555.24 \\
2.68\end{array}$ & $\begin{array}{r}0.47 \\
0.45 \\
443.17 \\
2.54\end{array}$ & $\begin{array}{r}0.47 \\
0.94 \\
242.00 \\
2.49\end{array}$ & $\begin{array}{r}0.37 \\
0.40 \\
408.04 \\
2.80\end{array}$ & $\begin{array}{r}0.48 \\
0.58 \\
396.18 \\
2.78\end{array}$ \\
\hline $\begin{array}{l}\mathrm{Tb} \\
\mathrm{In} \\
\mathrm{Sc} \\
\mathrm{Yb}(2)^{\mathrm{a}}\end{array}$ & $\begin{array}{l}0.52 \\
0.22 \\
5.40 \\
2.94\end{array}$ & $\begin{array}{l}0.78 \\
0.18 \\
4.58 \\
3.54\end{array}$ & $\begin{array}{l}0.79 \\
0.18 \\
9.19 \\
3.45\end{array}$ & $\begin{array}{l}0.45 \\
0.14 \\
4.54 \\
2.61\end{array}$ & $\begin{array}{l}0.66 \\
0.17 \\
5.65 \\
3.38\end{array}$ \\
\hline $\begin{array}{l}\mathrm{Ba} \\
\mathrm{Cr} \\
\mathrm{Lu}(2)^{\mathrm{a}} \\
\mathrm{Dy}\end{array}$ & $\begin{array}{r}187.10 \\
14.53 \\
0.44 \\
3.55\end{array}$ & $\begin{array}{r}257.94 \\
6.64 \\
0.52 \\
4.88\end{array}$ & $\begin{array}{r}256.69 \\
33.26 \\
0.50 \\
4.45\end{array}$ & $\begin{array}{r}249.53 \\
11.56 \\
0.39 \\
3.15\end{array}$ & $\begin{array}{r}291.25 \\
15.27 \\
0.50 \\
4.25\end{array}$ \\
\hline $\begin{array}{l}\mathrm{Fe} \\
\mathrm{Hf}\end{array}$ & $\begin{array}{r}7905.71 \\
3.07\end{array}$ & $\begin{array}{r}9440.56 \\
6.25\end{array}$ & $\begin{array}{r}23405.55 \\
6.54\end{array}$ & $\begin{array}{r}6611.09 \\
2.98\end{array}$ & $\begin{array}{r}10911.35 \\
4.55\end{array}$ \\
\hline
\end{tabular}


averages plotted in Fig. 2. This comparison indicates that this field is at about the 95 th percentile for US coals; that is, about $95 \%$ of the US coal seams have an average uranium content less than the average uranium content of these samples. However, in assessing the radiologic impact from these lignites, the ash content must also be considered. These samples contain $25 \%$ ash, which is about twice that found in the average US coal (13\%). Consequently, the ash of these lignites has a uranium content of $16 \mathrm{ppm}$, which is close to the US average, and the lignites are at the 75 th percentile on the probability curve for uranium in ash (Fig. 3). The average in Fig. 3 is found between the 50 th percentile (the median) and the 75 th percentile because of the skewness in a log-normal distribution. To put these results further into perspective, we note that the uranium and thorium content of the lignites is about the same as that of typical soils, ${ }^{1}$ and the content in the ash is about 4 times larger.

Consequently, we would expect these lignites, their waste products, and leachates to behave like other similar materials already reported in the literature.

\section{LEACHING TESTS}

Leaching tests were performed on samples of fly ash from two lignite-burning power plants (M-9 and M-11) in the northern Great Plains of the US. (See Refs. 1, 5, and 6 for further information on the fuel and waste materials from these plants.) These tests were done to determine the chemical character of the leachate, to measure the release of radionuclides by leaching, and to determine if the chemical nature of the leachate affected the leaching of the radionuclides. The radioactivity found in the leachates was quite small (in many samples it was below the detection limit), which precluded a detailed study of the effect of the leachate on the leachability of the radionuclides. In no case did the radionuclide levels in the raw leachates exceed the limits for drinking water by more than a factor of 3 (Ref. 7). Under usual field conditions, dilutions of raw leachates by a factor of 100 or more are expected. Although we can learn very little about the mechanism of leaching from studying these samples, the levels observed are too small to be of significant concern.

For the leaching tests, a $200-\mathrm{g}$ sample of ash was shaken with $1 \mathrm{~L}$ of deionized water in a 2-L polyethylene bottle. Samples of $200 \mathrm{~cm}^{3}$ of the leachate were withdrawn daily (except weekends), and $200 \mathrm{~cm}^{3}$ of fresh deionized water was added to the bottle to maintain the liquid volume. The leachate was filtered through a Whatman 541 filter, and the major cations were determined by inductively coupled plasma spectrophotometry. A 
calibrated $\mathrm{pH}$ meter was used to measure $\mathrm{pH}$ of the filtered leachate. The radionuclides were analyzed according to the procedures used in our previous studies ${ }^{1}$ (see Tables II and III).

The results for the stable species (see Figs. 4 through 9) follow four general types of behavior: Type I, followed by lithium, sodium, potassium, fluorine, and chlorine, starts at a particular lcvel and falls off gradually. Type II, followed by magnesium, calcium, and strontium, starts at a particular level, drops drastically after a few days, and then drops gradually thereafter. Type III, followed by aluminum, rises rapidly for the first few days and continues to rise, but more gradually, for the remainder of the run. Type IV, followed by silicon, borcn, vanadium, molybdenum, and barium, starts slowly, after a few days grows to a maximum, and falls off gradually.

Type I behavior would be expected from material that dissolves completely the first day. The gradual drop in concentration is caused solely by the process of withdrawing samples and adding fresh water, which dilutes the concentration. The material observed here probably comes from easily dissolved alkali metal salts. Type II behavior would be expected from material that is readily dissolved or suspended and that subsequently precipitates or forms larger solid particles. This behavior probably is due to finely divided lime particles or lime solutions that react and precipitate less soluble solid phases. Type III behavior would be expected from less soluble structural minerals that dissolve very slowly over a period of time longer than the duration of these tests. Type IV behavior would be expected from structural minerals such as silicates that dissolve in these very basic solutions over a few days. The gradual drop in concentration is expected from the dilution effect mentione $d$ above in the discussion of Type I behavior.

Radon-226 appears to follow Type IV behavior, which suggests that it resides in the struc:ural minerals, whereas $\mathrm{Pb}-210$ appears to follow Type I or II behavior, indicating a similarity to the alkalis or alkaline earths. We looked for several elements in addition to the above, but they were below the detection limits of the analytical scheme. These elements, with their detection limits in parts per million shown in parentheses, are copper (0.03), cadmium (0.3), titanium (0.003), iron (0.2), manganese (0.2), lead (0.7), cobalt $(0.1)$, and nickel $(0.1)$. The concentrations of these metais are controlled by hydroxide ion concentration at these high $\mathrm{pH}$ values (11.7 to 12.1), just as they are controlled in coal waste leachates treated with base. ${ }^{8}$

\section{ESTIMATION OF RELEASES FROM LIGNITES AND ASHES}

These lignites and ashes do not differ substantially from samples of similar material that we have studied during the past several years. The concentrations of radionuclides in 
TABLE II. Coal Fly Ash Leachate Compositions (ppm)

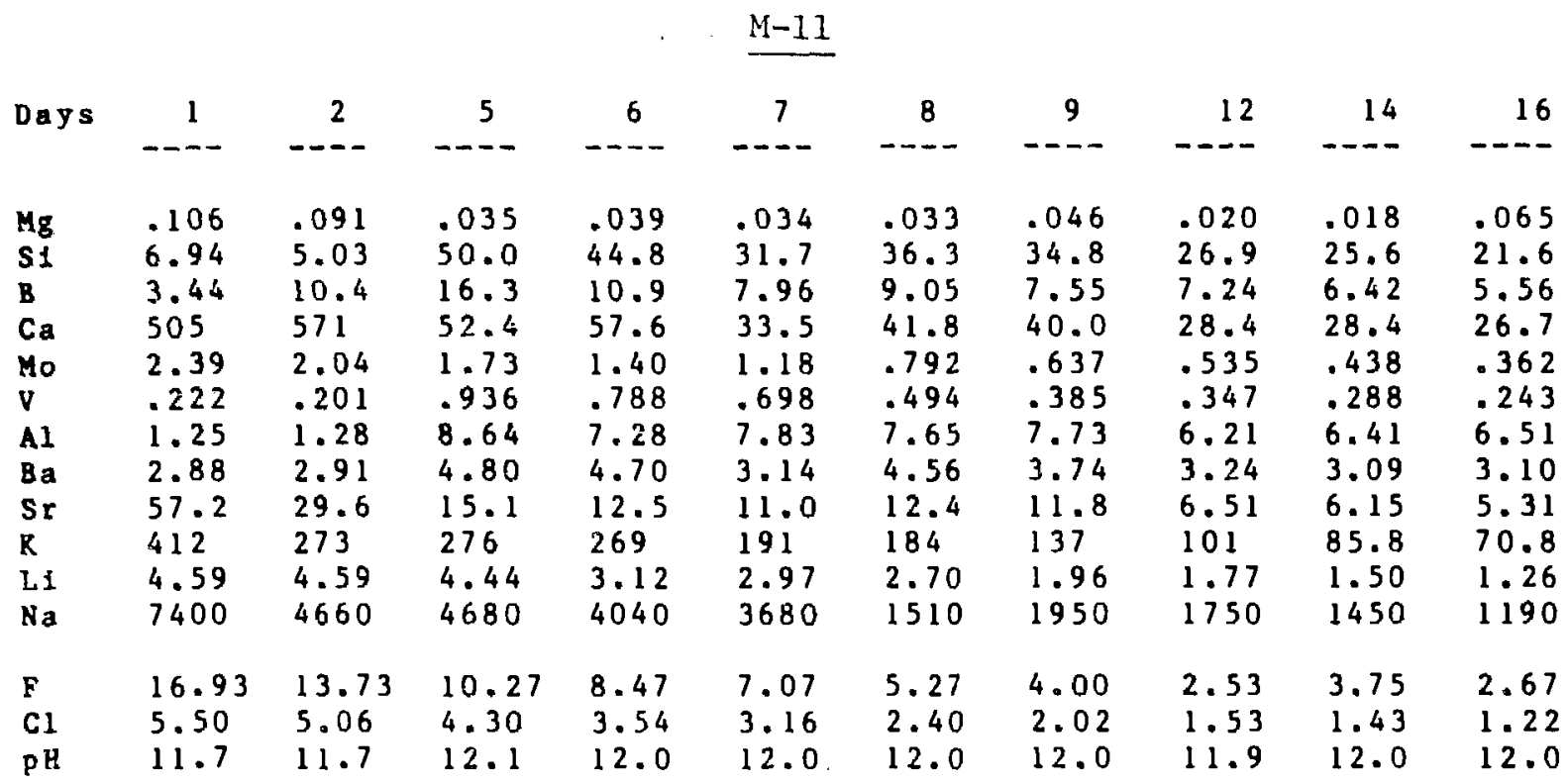

\section{$\underline{M-9}$}

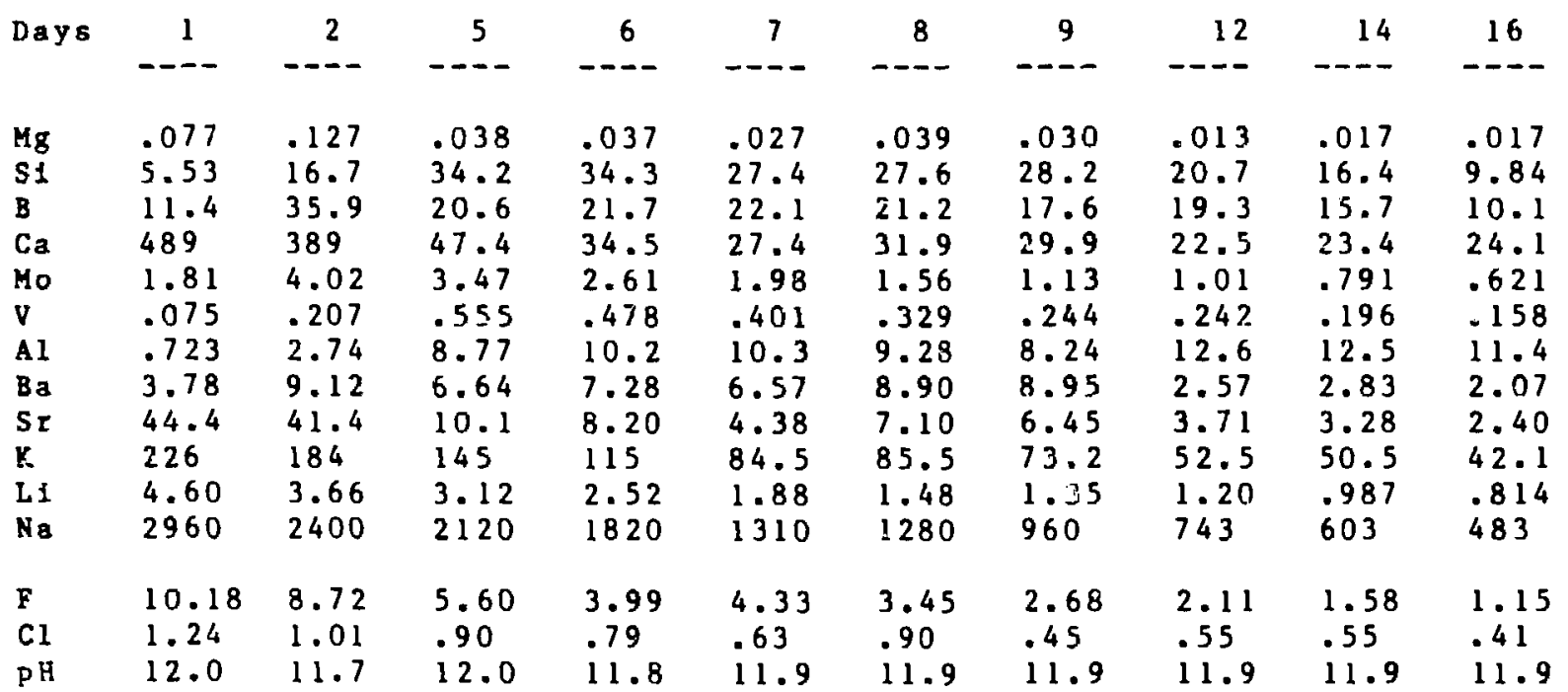

${ }^{\mathrm{a}} \mathrm{Cu}, \mathrm{Cd}, \mathrm{Ti}, \mathrm{Fe}, \mathrm{Mn}, \mathrm{Pb}, \mathrm{Co}$, and $\mathrm{Ni}$ were below detection limits. 
TABLE III. Fly Ash Leachate (pCi/L)

$\underline{M-11}$

$\begin{array}{llllllllllll} & 1 & 2 & 5 & 6 & 7 & 8 & 9 & 12 & 14 & 16 & \text { Det. Limit } \\ \mathrm{Ra}^{226} & - & - & - & 0.6 & - & 0.7 & - & - & 0.5 & - & 0.1 \\ \mathrm{~Pb}^{210} & - & 4.2 & 3.1 & 2.4 & 2.9 & 0.6 & 3.7 & - & 2.0 & 2.0 & 0.2 \\ \mathrm{Po}^{210} & - & - & - & 0.6 & - & - & - & - & - & - & 0.5\end{array}$

\section{$\underline{M-9}$}

\begin{tabular}{|c|c|c|c|c|c|c|c|c|c|c|c|}
\hline $\mathrm{Ra}^{226}$ & - & - & - & 2.3 & 11.4 & - & - & - & - & - & 0.1 \\
\hline $\mathrm{Pb}^{210}$ & 3.5 & 1.3 & - & 0.6 & 0.4 & - & - & 1.5 & 2.7 & 0.4 & 0.2 \\
\hline $\mathrm{Po}^{210}$ & 0.6 & - & - & - & - & - & - & - & - & - & 0.5 \\
\hline
\end{tabular}

these samples and their leachates fall within the range of values found previously. Consequently, on the basis of the available data, we expect that radionuclide releases from the materials in this study will not differ substantially from those already studied, provided that the conditions to which these materials are subjected are the same. Since these samples do not differ substantially from those discussed at great length in our previous reports, ${ }^{1,2,5,6,9,10}$ we will merely summarize the previous discussions, which used as reference cases those of Beck et al. ${ }^{4}$ Those cases indicate that airborne releases of radionuclides associated with fly ash from a modern 1000-MW(e) coal-fired (or lignite-fired) power plant will result in an increased dose in the maximally exposed individuals of about $0.14 \mathrm{mrem} / \mathrm{yr}$, which is $0.1 \%$ of the normal background dose. Doses from leached radionuclides from solid waste materials generated in the coal power cycle are generally much smaller than those from airborne dose. Numerous assumptions are made to estimate these doses. Beck et al. authoritatively discuss the assumptions, the basic data, and the resulting estimates. $^{4}$ The extensive data we have gathered since the publication of Beck et al. strengthen their assumptions and extend the data base. ${ }^{2,9}$ None of the new results, includ . ing those of this study, vary from the conclusions of Beck et al.

The case cited above ${ }^{4}$ was based on the assumptions of average radionuclide concentrations and a modern power plant. We have extended the discussion to include the distribution of radionuclide concentrations expected in the US coal supply for power generation 
and the distribution of emissions reported for the entire US population of power generation sites. 11 Because of generation capacities much greater than $1000 \mathrm{MW}(\mathrm{e})$ and emissions per megawatt greater than those assumed by Beck et al, a few multiple-plant sites will present doses considerably higher than those cited above. However, in this analysis only about $1 \%$ of the power-generating sites in the US would be expected to exceed a dose of $5 \mathrm{mrem} / \mathrm{yr}$ to the maximally exposed individuals. This dose is about $3 \%$ of the natural background dose.

\section{CONCLUSIONS}

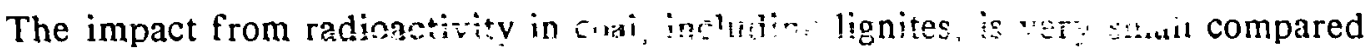
with that from natural background radiation. There are indications ${ }^{9}$ that small portions of the US coal supply may contain radionuclide concentrations considerably higher than the average US coal. However, in spite of our best efforts, we were unable to obtain samples with more than 3 times the average radionuclide concentration. (The samples in this study were only 2 times the average.) The only possible way for radioactivity in coal to become a problem is to burn very radioactive coal (a small probability) as the principal source of fuel at a site with very high emissions (a diminishing probability as plants become modernized). For $99 \%$ or more of the US coal-fired power plants, there should be very little problem from solid radionuclides or their leachates.

\section{ACKNOWLEDGMENTS}

The authors thank L. E. Wangen for indispensable assistance with the multivariate data analyses and E. J. Peterson and R. C. Heaton for many useful discussions during this study. We also are grateful to $J$. Cunningham for the leaching experiments, V. T. Hamilton for the spectrophotometry on the leachates, J. Bubernak and V. Hndge for the radionuclide analyses of the leachates, M. Minor and S. Garcia for the neutron activation analyses, and S. Houidobre for sample preparation.

\section{REFERENCES}

1. Paul Wagner and N. Roy Greiner, "Third Annual Report, Radioactive Emissions from Coal Production and Utilization, October 1, 1980--September 30, 1981," Los Alamos National Laboratory report LA-9359-PR (June 1982).

2. N. Roy Greiner and Paul Wagner, "Radioactive Emissions from Coal Production and Utilization: The Typical Case Put into Perspective," Los Alamos National Laboratory report LA-9747-PR (August 1983).

3. National Council on Radiation Protection and Measurements, Natural Background Radiation in the United States (NCRP Publication 45, Washington, DC, 1975). 
4. H, L. Beck, C. V. Gogolak, K. M. Miller, and W. M. Lowder, "Perturbations on the Natural Radiation Environment Due to the Utilization of Coal as an Energy Source," in Natural Radiation Environment III, Vol. 2, Thomas F. Gesell and Wayne M. Lowder, Eds. (Technical Information Cenier/US Department of Energy, Washington, DC), pp. $1521-1556$.

5. Paul Wagner, David R. Dreesen, Eugene M. Wewerka, and N. Roy Greiner, "Radioactive Emissions from Caal Production and Utilization, July I, 1978-September 30, 1979," Los Alamos National Laboratory report LA-8618-PR (December 1980).

6. Paul Wagner and N. Roy Greiner, "Second Annual Report, Raulioactive Emissions from Coal Production and Utilization, October 1, 1979--September 30, 1980," Los Alamos National Laboratory repoit LA-8825-PR (July 1981).

7. "Radioactivity in Drinking Water," Environmental Protection Agency report EPA-570/4-81-002 (1981).

8. R. C. Heaton, L. E. Wangen, P. L. Wanek J. M. Williams, E. F. Thode, M. M. Jones, A. M. Nyitray, P. Wagner, and J. P. Bertino, "Trrace Element Characterization of Coal Wastes--Fif th Annual Progress Report, October 1, 1979--September 30, 1980," Los Alamos National Laboratory report LA-8826-PR (May 1981).

9. N. Roy Greiner, Michael D. Williams, and Paul Wagner, "Estimation of Radionuclide Releases from Specific Large Coal-Fired Industrial and Utility Boilers," Los Alamos National Laboratory report LA-9845-MS (August 1983).

10. N. Roy Greiner, "Radon Emanation from Coals: Effect of Moisture and Particle Size," Health Physics 48, 283 (1985).

i 1. N. Roy Groiner, Michael D. Williams, and Paul Wagner, "Radioactivity Releases During Coal Combuscion and the Distribution of Airborne Dose," submitted to Health Physics. 


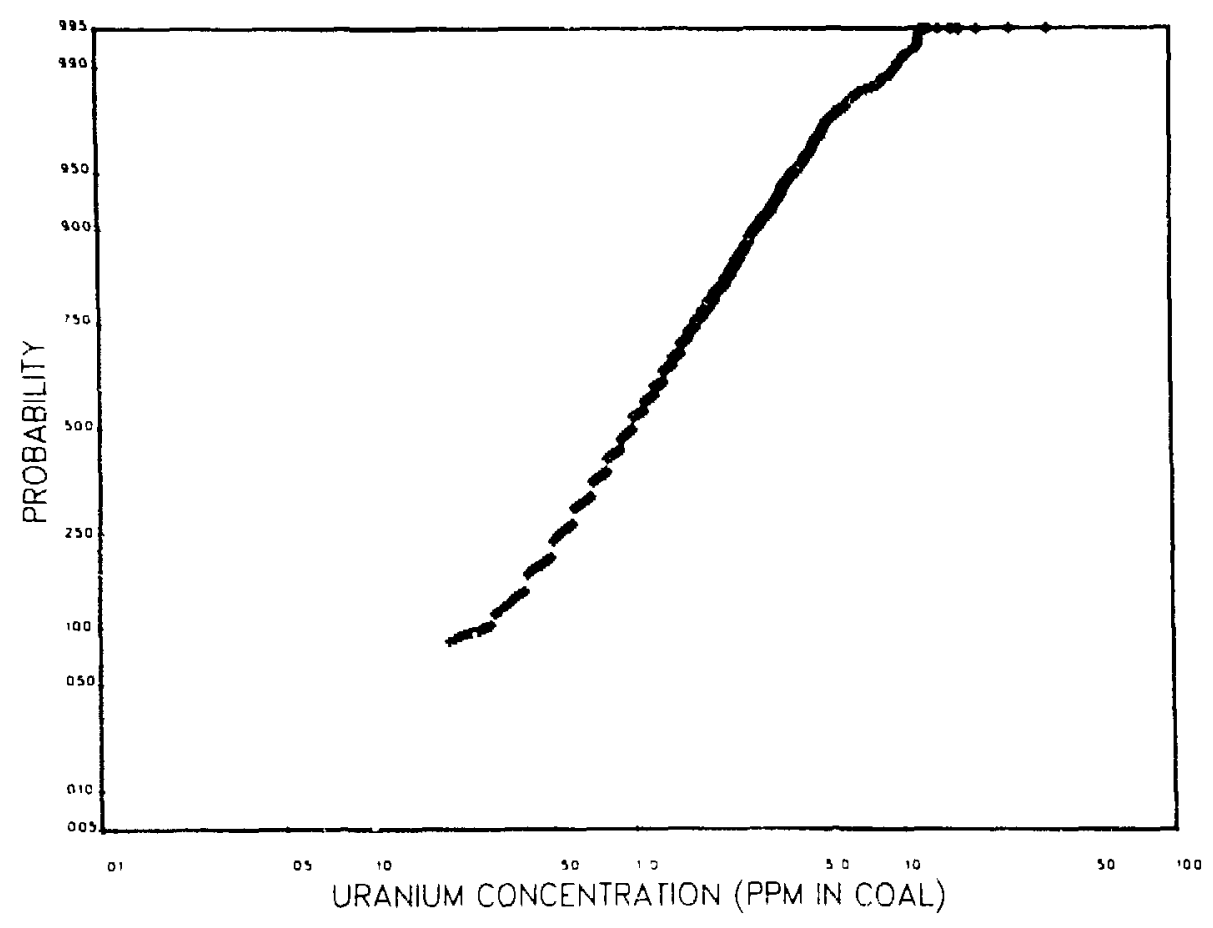

Fig. 1.

Cumulative probability curve for individual coal samples in the data base judged to be economic fuel coals. Probability scale is the fraction of the coals that have a uranium concentration equal to or less than the concentration value shown on the horizontal scale. Note that the corcentration scale is logarithmic.

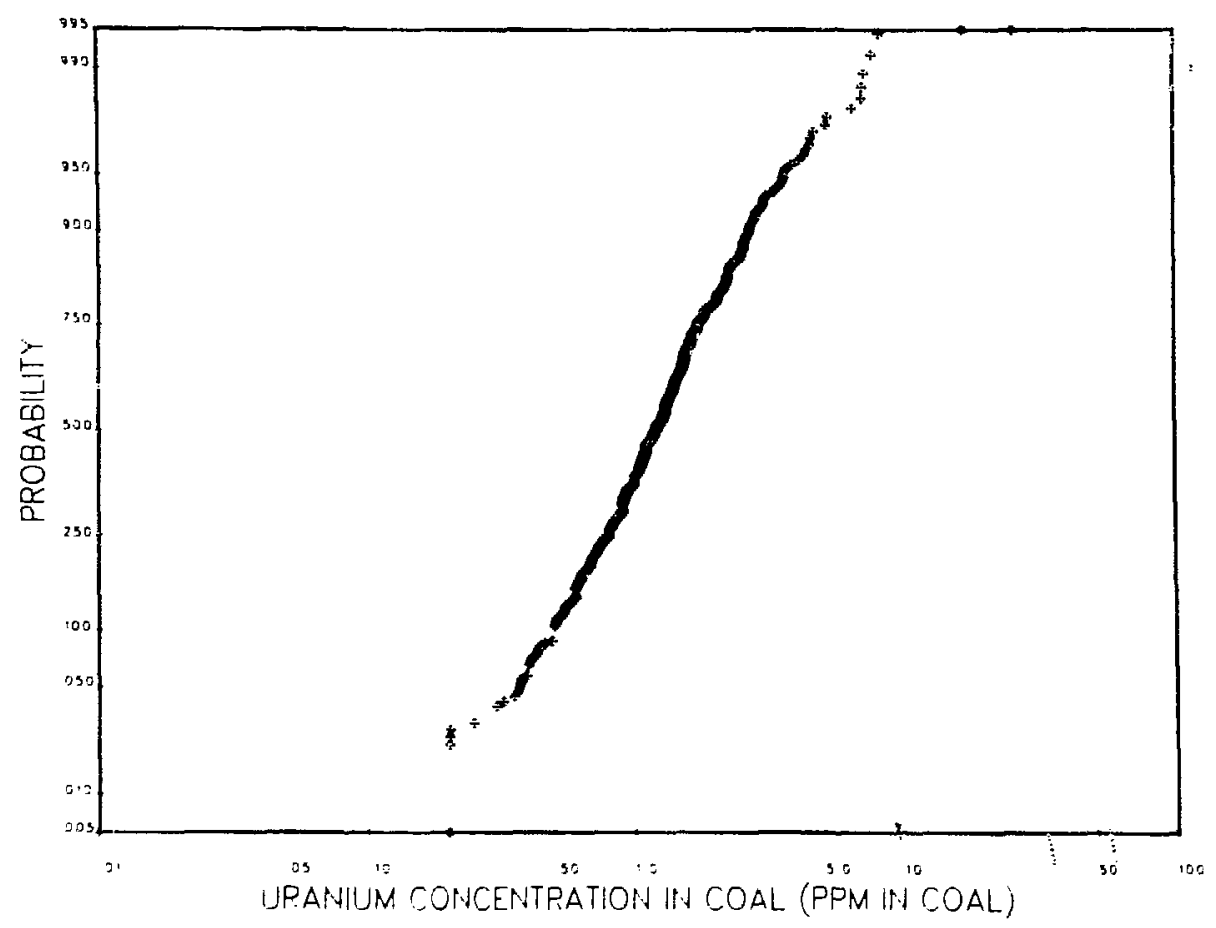

Fig. 2.

Cumulative probability curve for seam averages weighted by seam thickness. Probability scale is the fraction of the total seam thickness that has uranium concentrations equal to or less than the value indicated on the horizontal scale. 


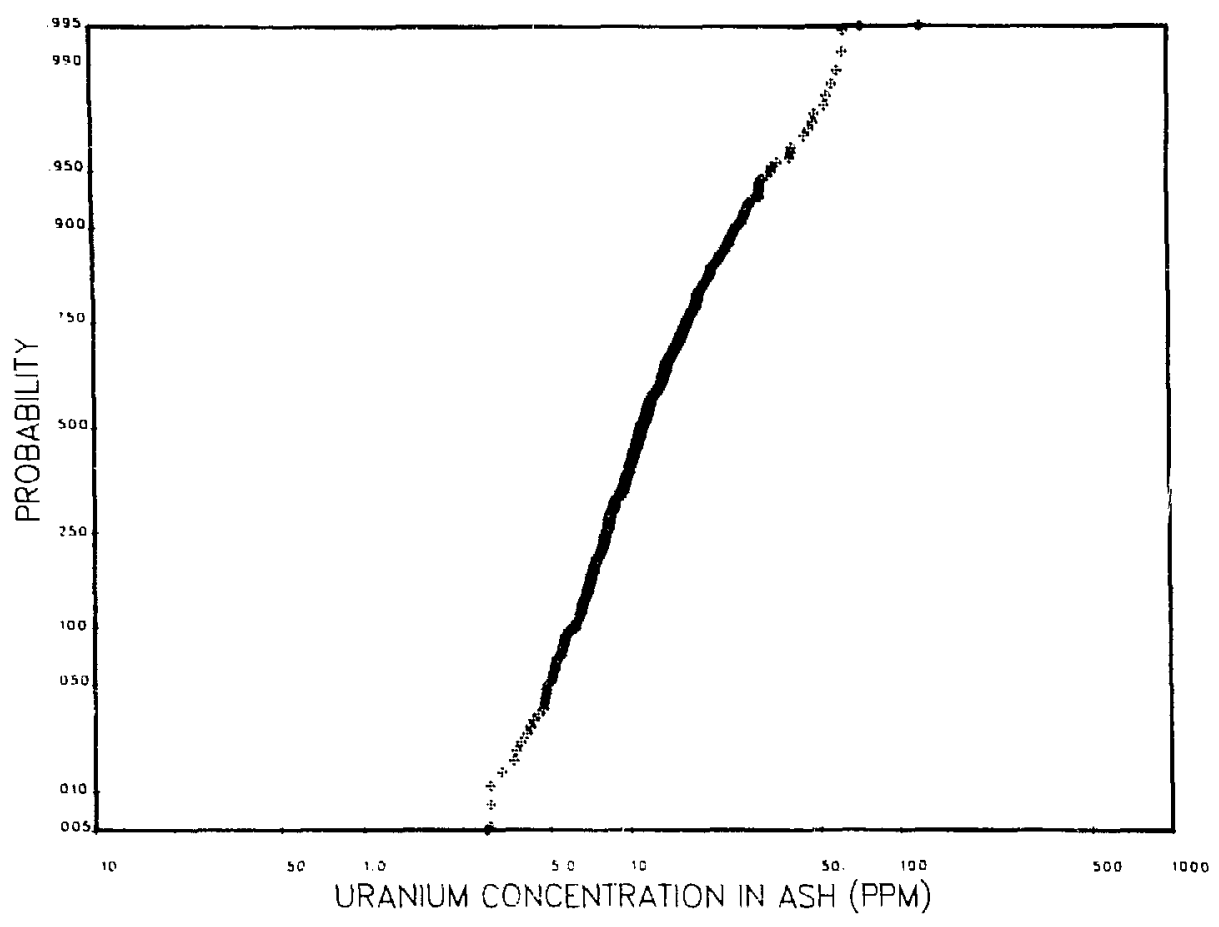

Fig. 3 .

Cumulative probability curve for the uranium content of coal ash. The uranium content of the ash for each sample was obtained by dividing the uranium content of the coal by the ash content of the coal. Seam averages were obtained by weighting the ash content of each sample by the seam thickness of each sample. This probability scale is the fraction of the total seam thickness of all seams that have a uranium concentration equal to or less than the value indicated on the horizontal scale. 

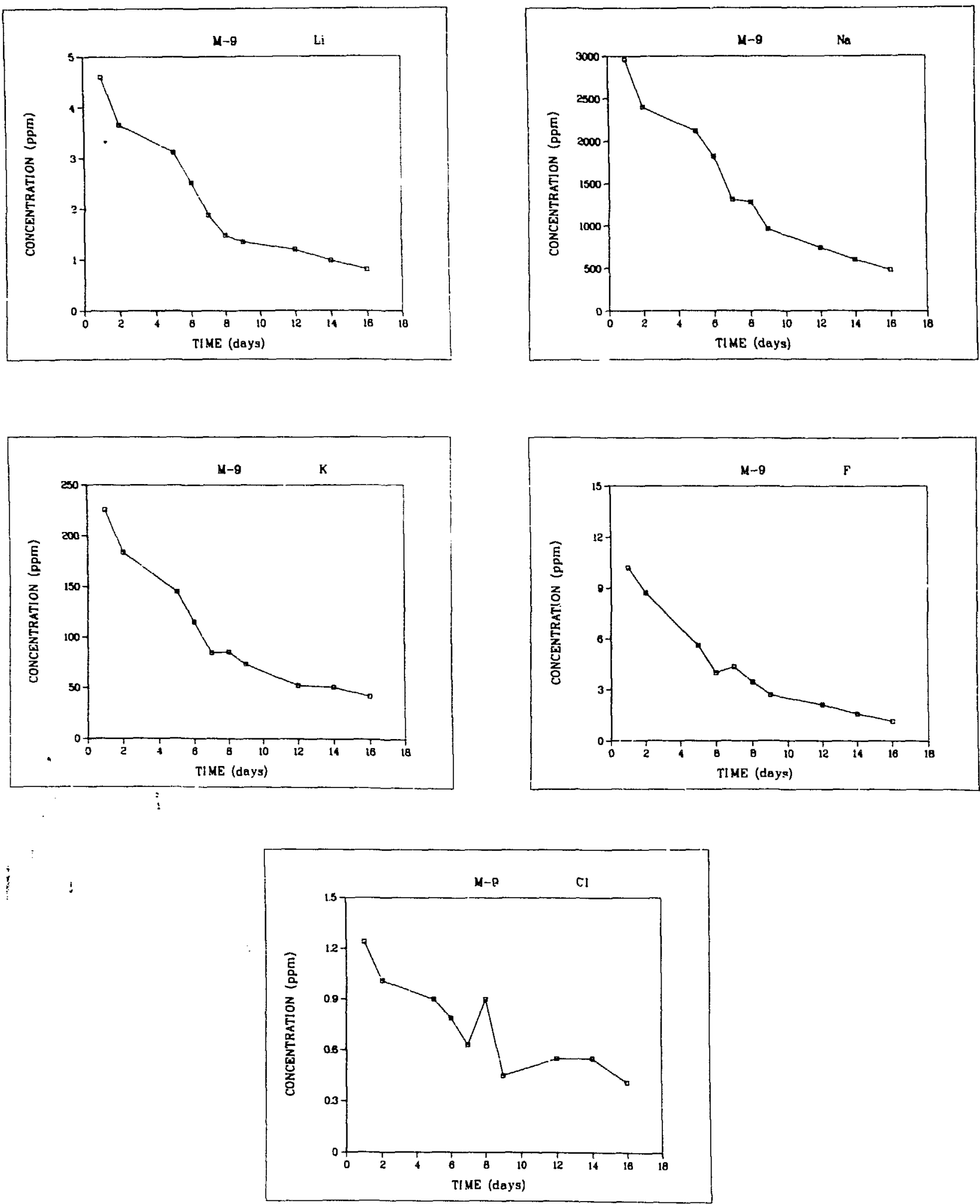

Fig. 4.

Elements showing Type I behavior (lithium, sodium, potassium, fluorine, and chlorine) in leachates of M-9 ash. 

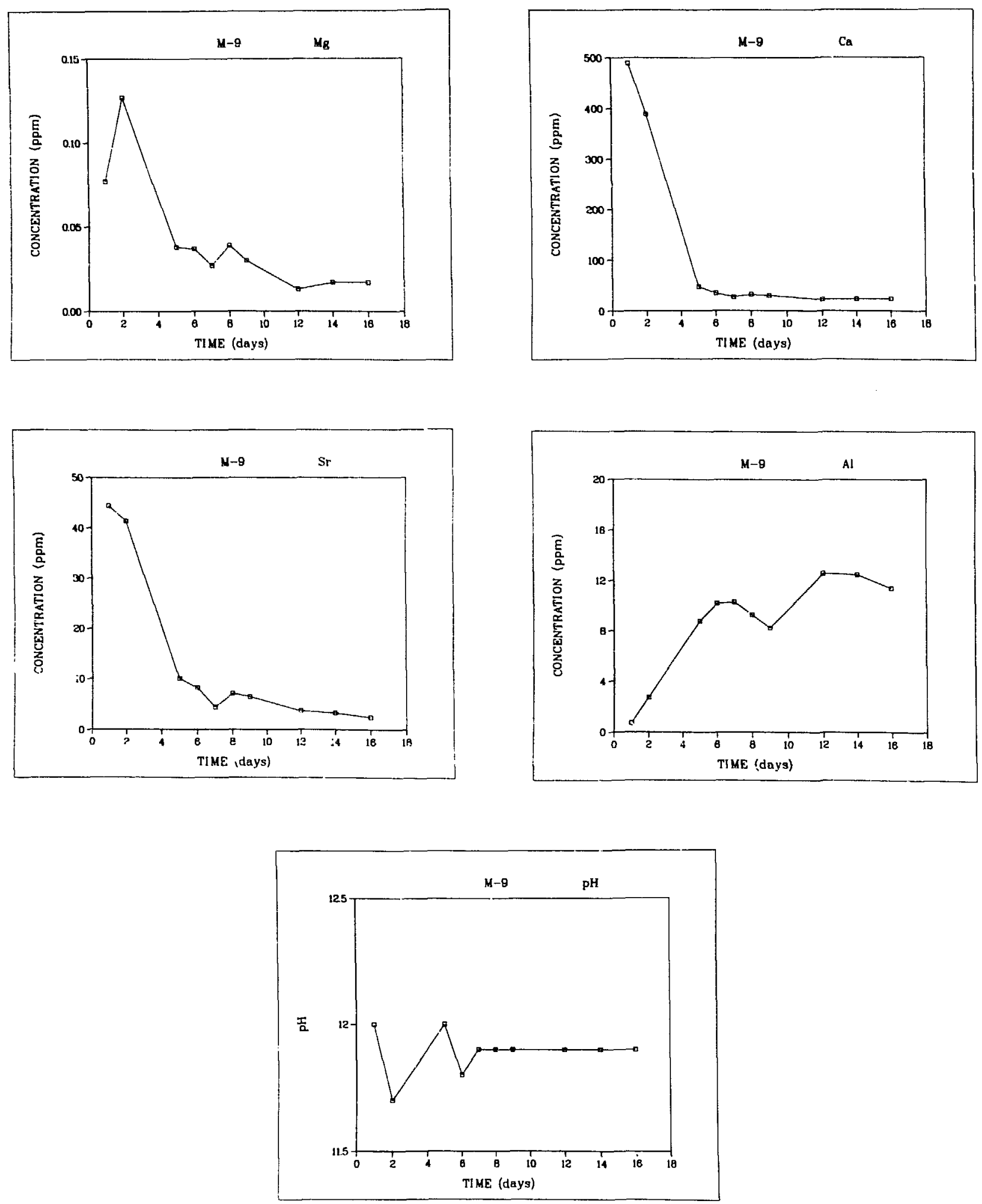

Fig. 5.

Elements showing Type II behavior (magnesium, calcium, and strontium) and Type III behavior (aluminum) and $\mathrm{pH}$ in leachates of $\mathrm{M}-9$ ash. 

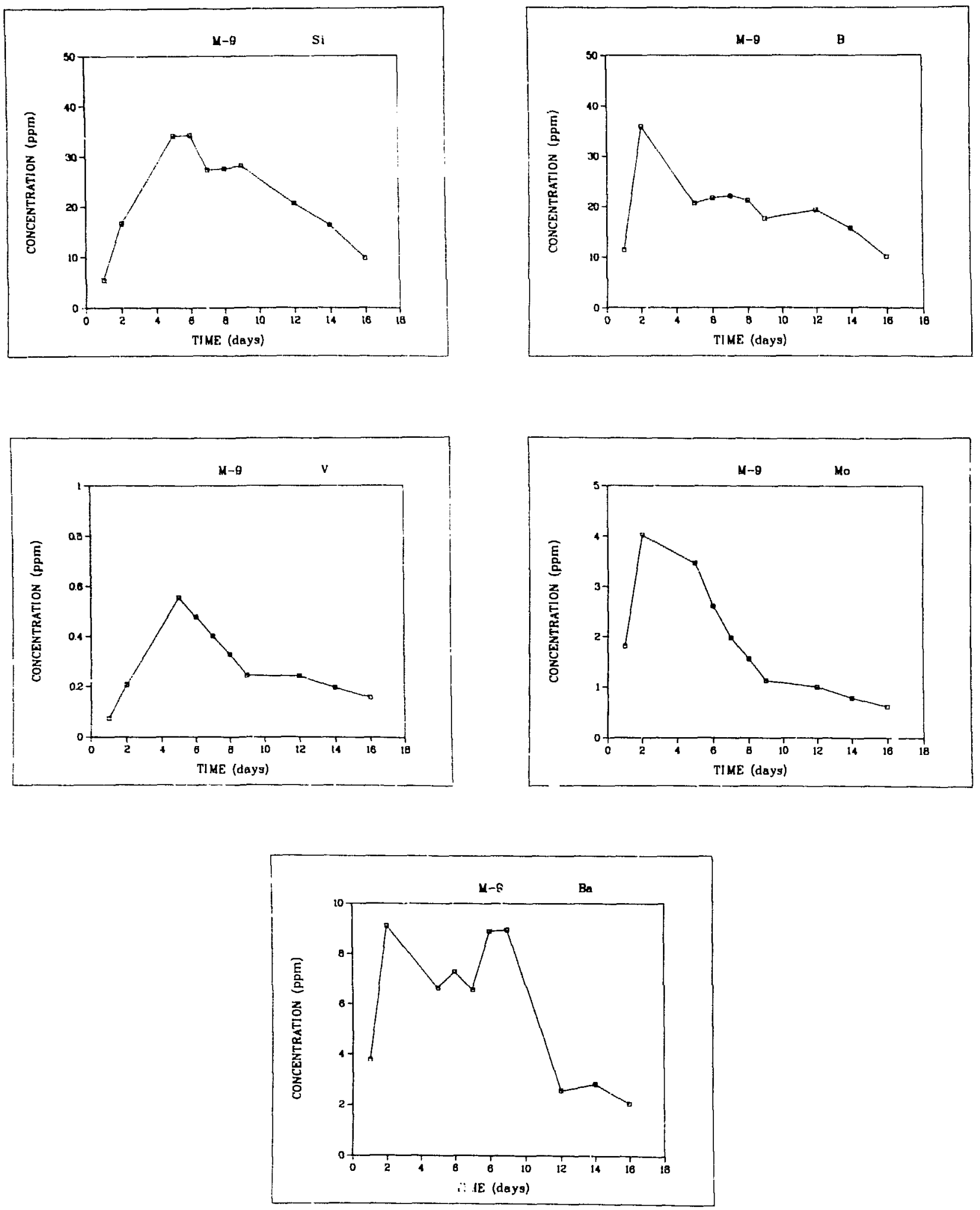

Fig. 6.

Elements showing Type IV behavior (silicon, boron, vanadium, molybdenum, and barium) in leachates of M-9 ash. 

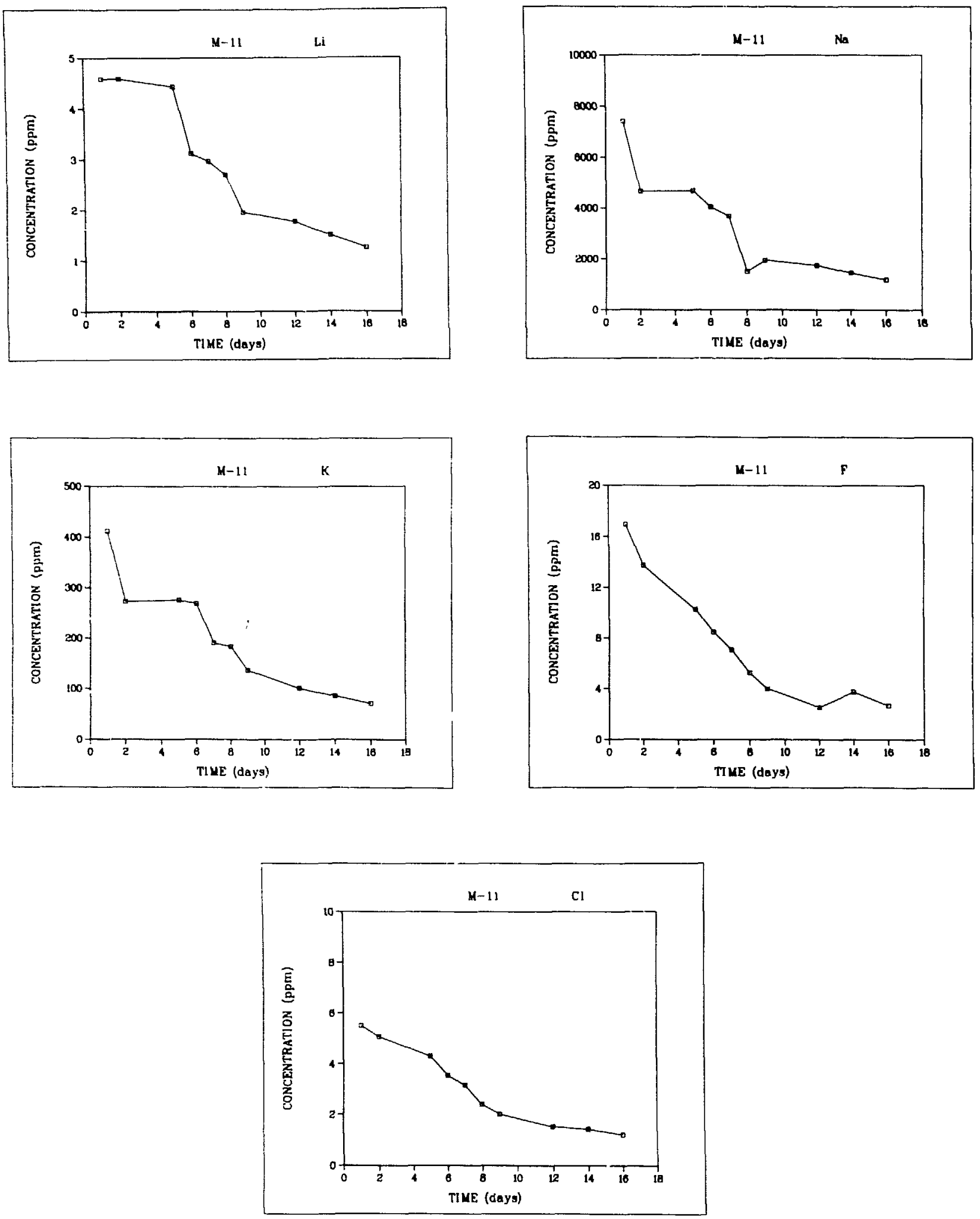

Fig. 7.

Elements showing Type I behavior (lithium, sodium, potassium, fluorine, and chlorine) in leachates of $\mathrm{M}-11$ ash. 

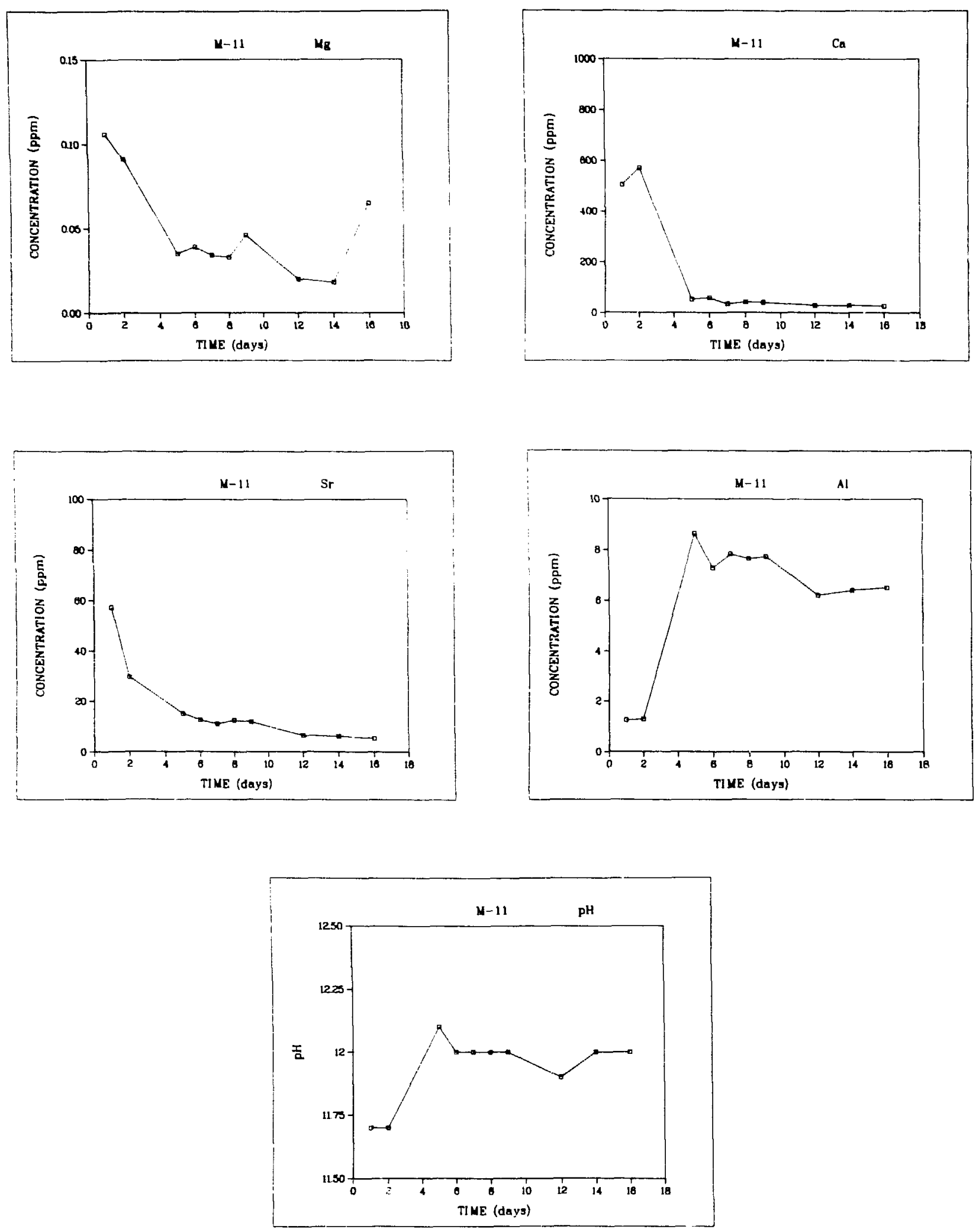

Fig. 8.

Elements showing Type II behavior (magnesium, calcium, and strontium) and Type III behavior (aluminum) and $\mathrm{pH}$ in leachates of $\mathrm{M}-11$ ash. 

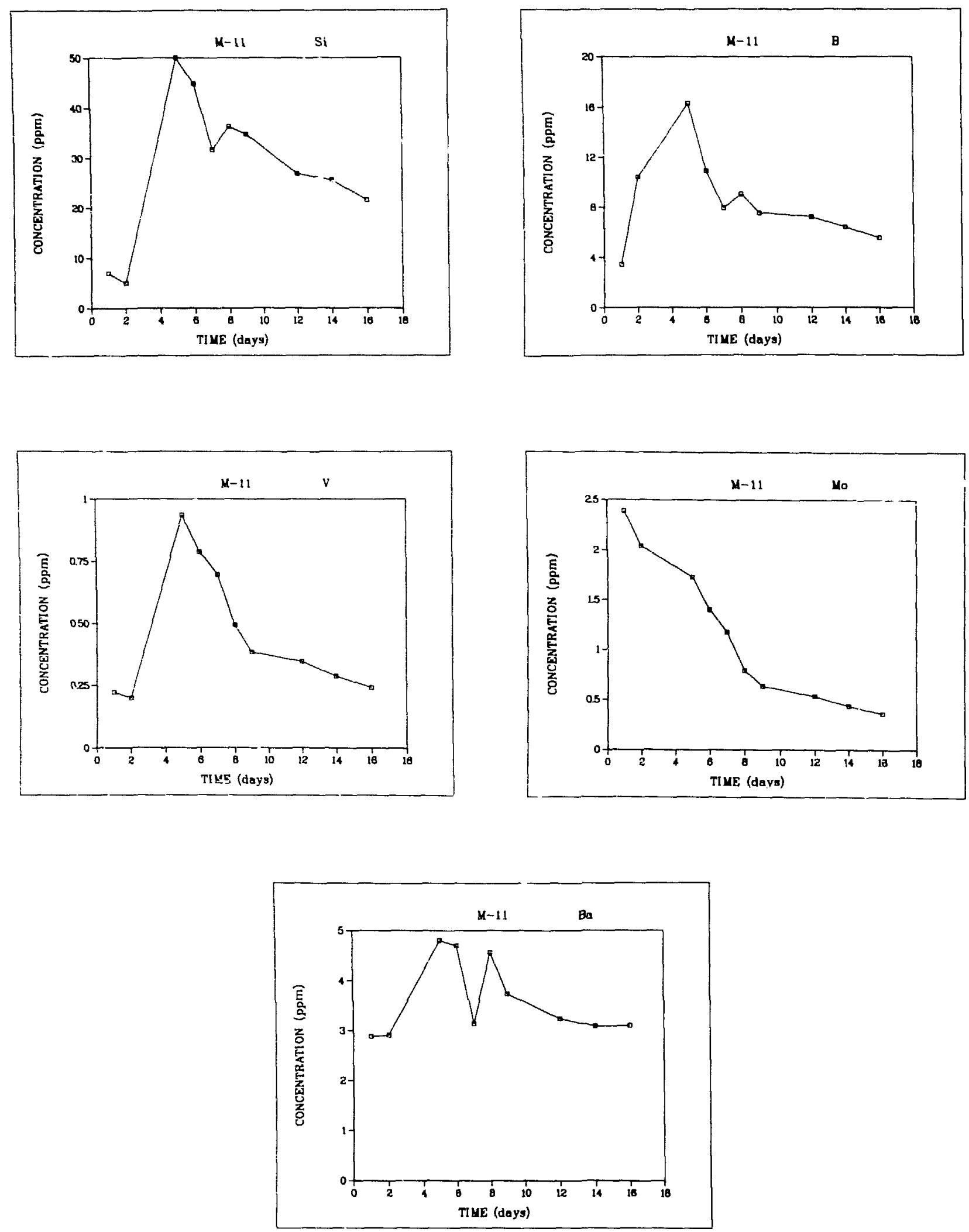

Fig. 9.

Elements showing Type IV behavior (silicon, boron, vanadium, molybdenum, and barium) in leachates of M-11 ash. 


\section{APPENDIX}

RSELTS OF NEUTRON ACTIVATION ANALYSIS 


$\begin{array}{lc}\text { FILE ID } & 6100450 \\ \text { SAMPLE } & 001 \\ \text { DUPLICATE } & 051 \\ \text { CLUSTER } & 09 \\ \text { TYPE } & L\end{array}$

ELEMENT

$\mathrm{Na}-24$

$\mathrm{Mg}-27$

Al - 28

Cl - 38

$\mathrm{K}-42$
$\mathrm{Ca}-4 \mathrm{~g}$

$\mathrm{Ca}-49$
$\mathrm{~T} i-51$

$v-52$

Mn- 56

$\mathrm{Cu}-66$
$\mathrm{Sn}=\mathrm{B7}$

In- 116

I -128

Ba- 139

Dy -165

U -235
$\mathrm{Ga}-72$

$\mathrm{Ga}-72$
$\mathrm{As}-76$

$\mathrm{Br}-\mathrm{B2}$

Mo- 99

$5 \mathrm{c}-122$
$\mathrm{~L}-140$

$S: 1-153$

YO- 175

LU - 177

$w-187$
$\mathrm{Au}-198$

Sc- 46

Cr- 5

Co 59

Zn- 65

$2 n-65$

Rb- 86

$\mathrm{Rb}-86$
$\mathrm{Zr}-95$

$\mathrm{Ag}-110$

Sb-124

Cs -134

Ba-131

Ce-141

$\mathrm{No}-147$

Tb-160

Yb- 169

Hf -181

Ta-182

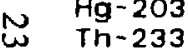

6100470

003

L
6100480

004

04

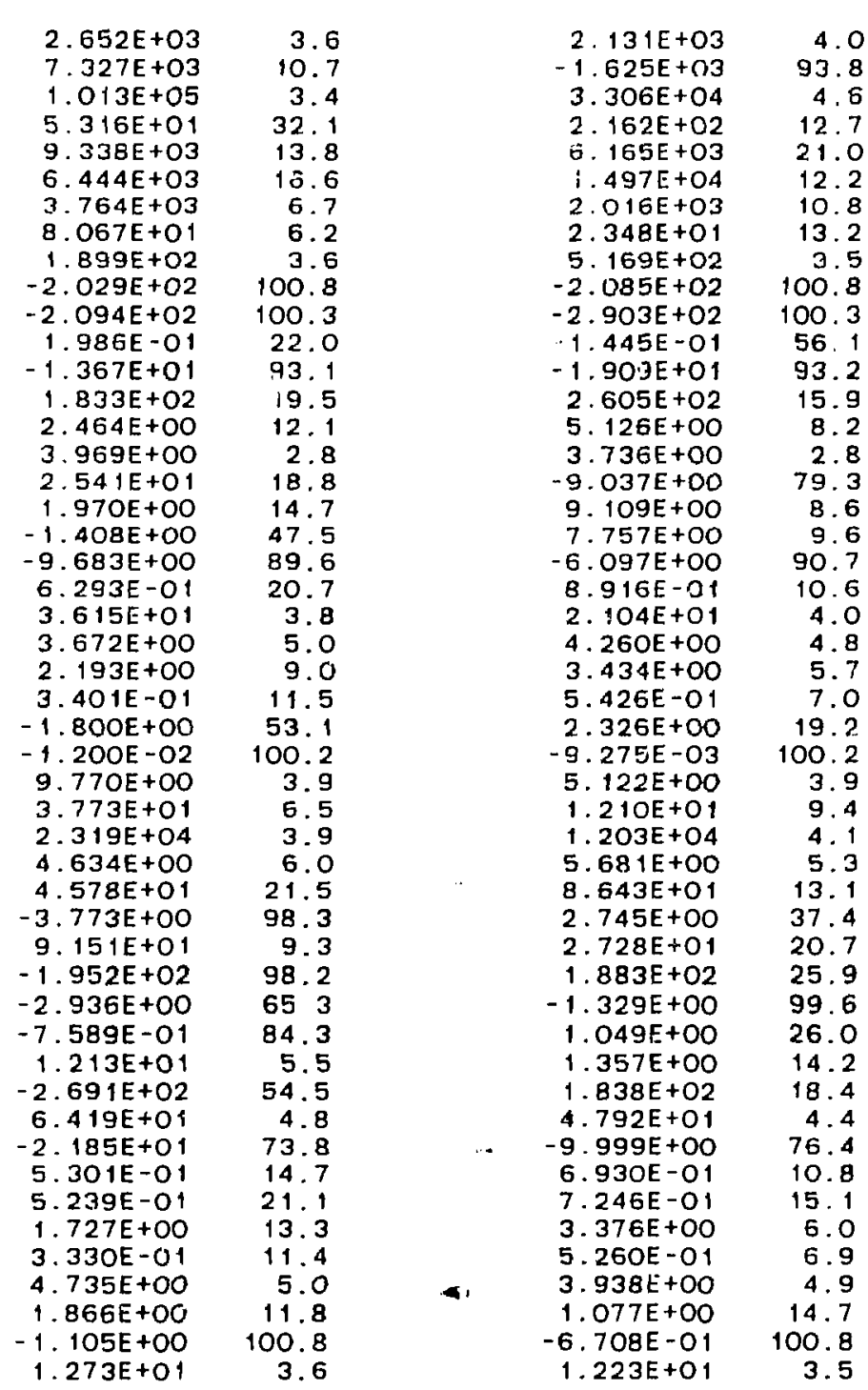

$\begin{array}{rr}6.002 E+O 3 & 3.1 \\ 4.108 E+O 3 & 12.5 \\ 5.948 E+O 4 & 3.7 \\ 6.156 E+O 1 & 72.2 \\ 1.57 O E+O 4 & 10.0 \\ 3.158 E+O 3 & 21.9 \\ 3.267 E+O 3 & 7.0 \\ 4.991 E+O 1 & 7.3 \\ 2.049 E+O 2 & 3.6 \\ -2.012 E+O 2 & 100.8 \\ 1.917 E+O 2 & 100.3 \\ 1.172 E-O 1 & 55.9 \\ 1.274 E+O 1 & 93.1 \\ 4.476 E+O 2 & 8.9 \\ 9.799 E+O O & 6.8 \\ 3.117 E+O O & 2.8 \\ 9.824 E+O O & 28.5 \\ 9.646 E+O O & 8.4 \\ 1.434 E+O O & 47.8 \\ 7.912 E+O O & 90.9 \\ 1.130 E+O O & 11.4 \\ 5.717 E+O 1 & 3.6 \\ 9.398 E+O O & 4.6 \\ 5.409 E+O O & 4.8 \\ 8.395 E-O 1 & 5.3 \\ 2.007 E+O O & 54.7 \\ 1.209 E-O 2 & 100.2 \\ 7.159 E+O O & 3.9 \\ 3.4 O 4 E+O 1 & 6.8 \\ 2.243 E+O 4 & 3.9 \\ 8.152 E+O O & 4.8 \\ 5.441 E+O 1 & 17.6 \\ 4.353 E+O O & 101.4 \\ 9.087 E+O 1 & 8.9 \\ 2.820 E+O 2 & 25.1 \\ 2.425 E+O O & 65.5 \\ 9.002 E-O 1 & 31.9 \\ 6.615 E+O O & 6.4 \\ 4.501 E+O 2 & 13.4 \\ 1.209 E+O 2 & 3.8 \\ 8.059 E+O 1 & 21.0 \\ 1.743 E+O O & 7.4 \\ 1.301 E+O O & 10.8 \\ 6.107 E+O O & 5.0 \\ 8.234 E-O 1 & 5.8 \\ 1.034 E+O 1 & 3.7 \\ 1.177 E+O O & 13.3 \\ 9.601 E-O 1 & 100.8 \\ 1.245 E+O 1 & 3.5\end{array}$




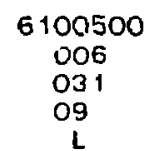

L

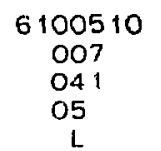

L
6100520

008

052

\section{ELEMENT}

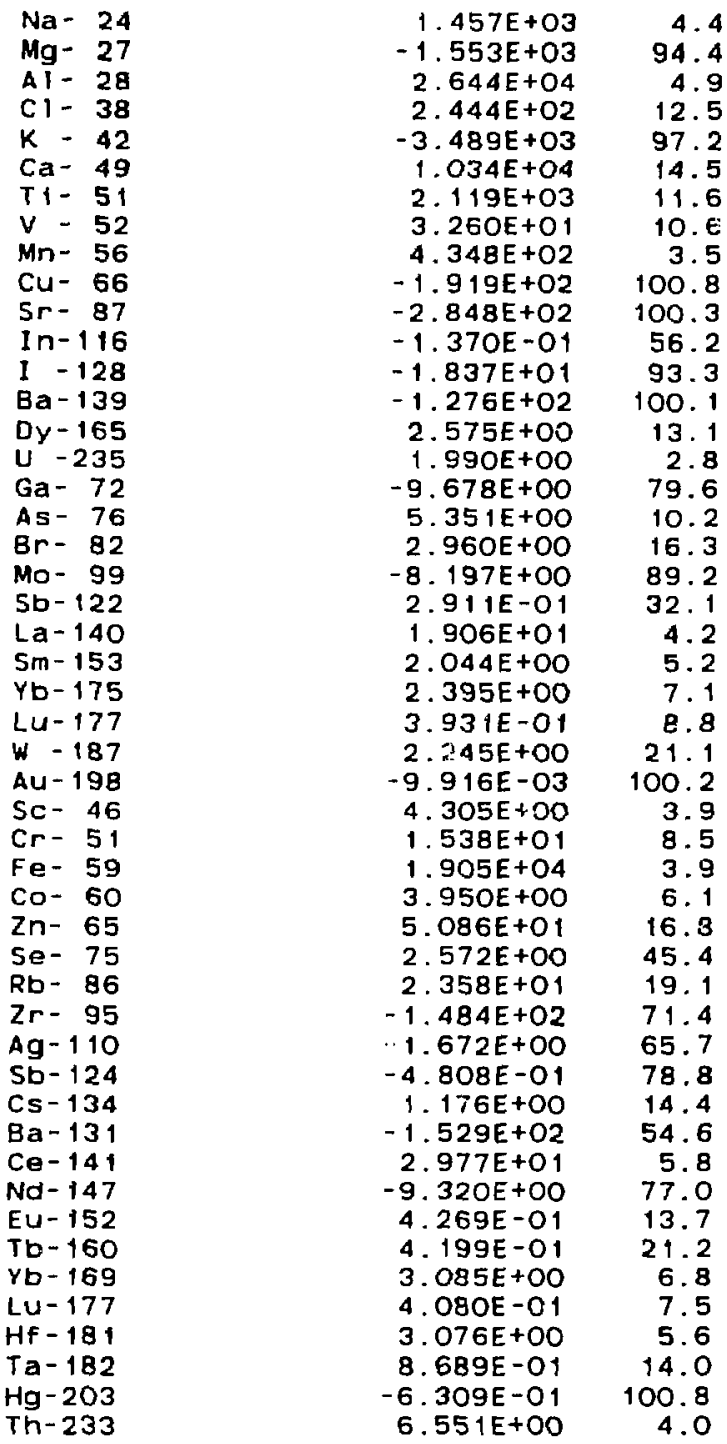

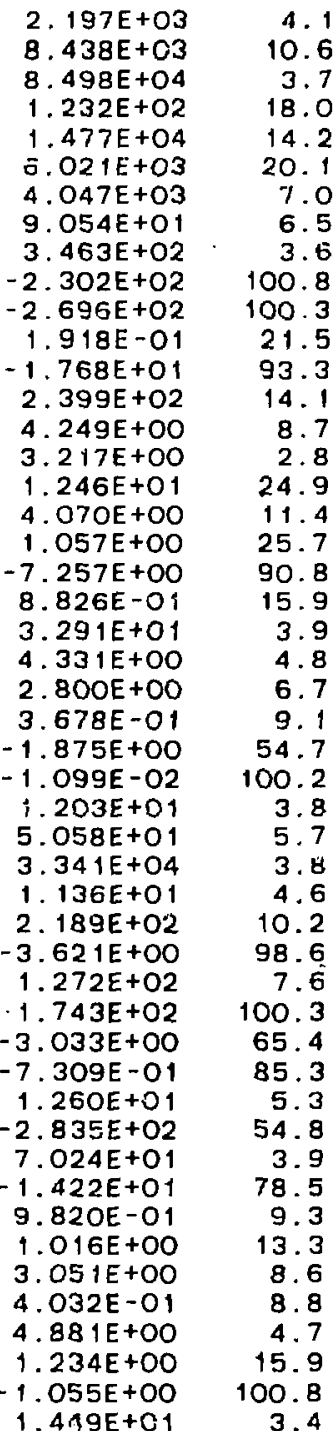

$1.180 E+03$

$-1.976 E+03$

3. $436 \mathrm{E}+04$

$4.598 \mathrm{E}+\mathrm{O} 3$

1. 599E + 04

$7.344 E+O 2$

2. $232 E+O 1$

2. $457 E+02$

$-2.457 E+02$
$-3.55 O E+O 2$

-3. $550 E+02$

2.674E-O

$-1.408 E+02$

3. $637 E+0 O$

3. $880 E+O O$

- B $714 E+00$

B. $714 E+O O$

4. $856 E+00$

.

6. $268 E+O O$

1. $227 E+O O$

1. $477 E+01$

2. $698 E+O O$

2. $723 E+O O$

4. $518 E-O 1$
2. $655 E+O O$

$-8.322 \mathrm{E}-03$

4. $255 E+O O$

1. $166 E+01$

1. $068 E+04$

$.686 E+00$

4. $564 E+01$

3. $17 \mathrm{OE}+O O$

- $1.044 E+01$

$-1.557 E+02$

$-1.771 \mathrm{E}+0 \mathrm{O}$

$-6.542 \mathrm{E}-\mathrm{O} 1$

7. $325 \mathrm{E}-01$

$-1.542 E+02$

$3.096 E+01$

$-1.052 E+01$

4. 223E-O1

4. 893E-01

$2.830 E+00$

$4.075 E-01$

2. $528 E+O 0$

$\begin{array}{rrr}1.290 E+O 0 & 15.0 & 8.18 B E-01 \\ 1.023 E+00 & 100.8 & -6.835 E-01 \\ 1.418 E+01 & 3.6 & 1.221 E+01\end{array}$

$\begin{array}{rrr}1.290 E+O 0 & 15.0 & 8.18 B E-01 \\ 1.023 E+00 & 100.8 & -6.835 E-01 \\ .418 E+01 & 3.6 & 1.221 E+01\end{array}$
5.2
93.7

4

4.9
13.3

97.1

14.9

94.9

$15 \cdot 8$

100.8

100.3

25.5

100.1

9.9

2.8
79.1

10.5

10.5

22.2

0. 1

4. 5

6.3

8.2

16.8

100.2

3.9
14.2

4.2

9.8

19.9

30.2

100.0

71.5

65.2

66.8

28.4

56.9

5.2
76.0

15.7

24.9
7.6

8.2

6.3

20.0

100.8
3.6 


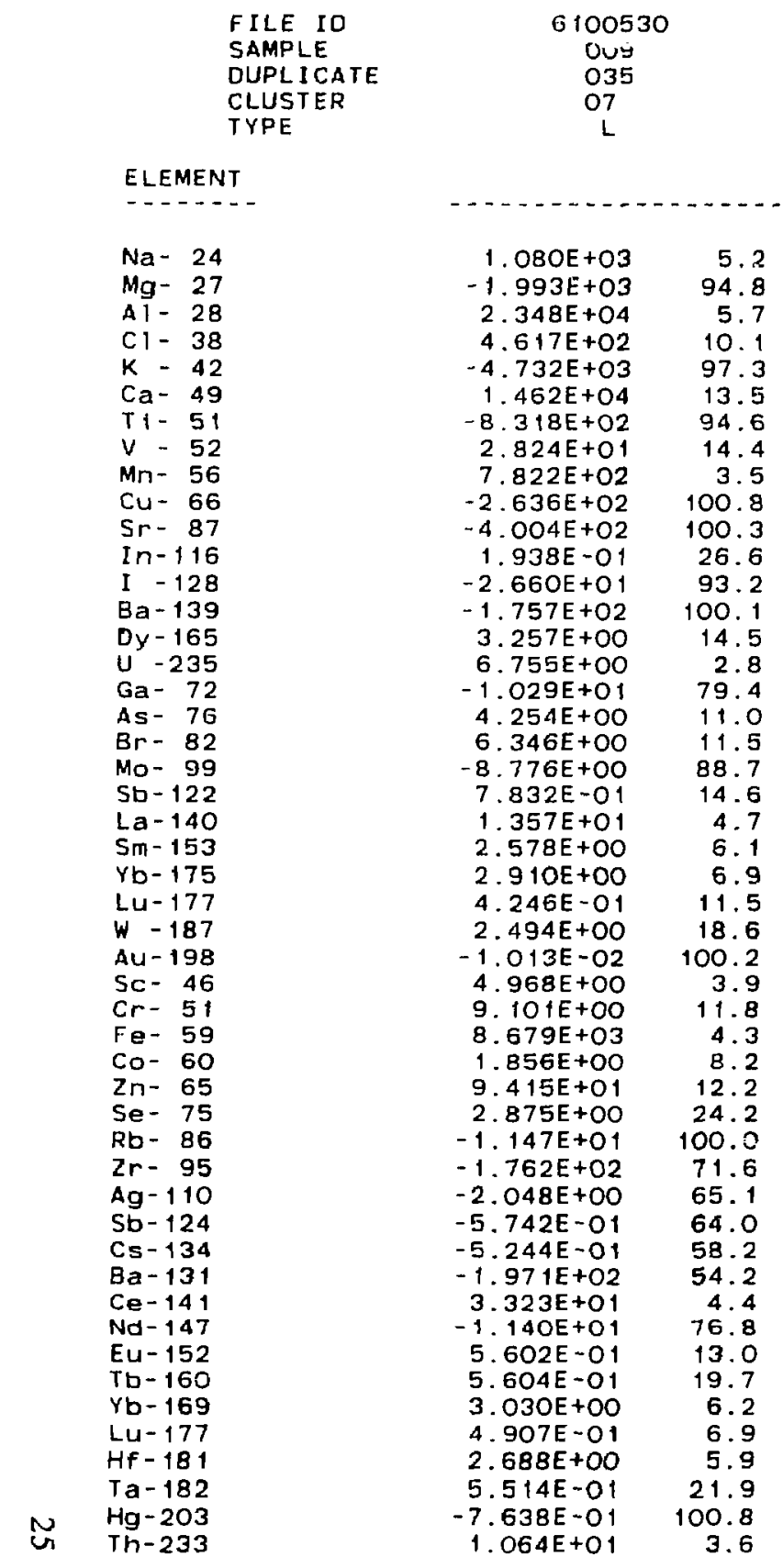

6100540

010

OB
6100550

011

10
6100560

012

07
$-2.707 E+03$

8. $399 E+O 4$

9. $146 \mathrm{E}+02$

3. 166E +

1. $205 E+03$

8. $333 E+00$

$3.461 E+02$

$-3.476 \mathrm{E}+02$

- $3.224 E+O 2$

$-2.04 O E-01$

2. $109 E+O$

2. $221 \mathrm{E}+\mathrm{O} 2$
6. $216 \mathrm{E}+\mathrm{OO}$

6. $216 \mathrm{E}+00$

2. $056 \mathrm{E}+\mathrm{O}$

$6.3 .42 E+00$

2. $0 ; 8 E+O C$

1. $119 \mathrm{E}+\mathrm{O}$

1. $539 E+O O$

4. $009 E+01$

$5.802 E+00$

4. $120 E+O O$

2. $659 E+00$

2. $659 E+00$

$-1.803 E-02$

3. $448 E+O O$

9. $382 \mathrm{E}+\mathrm{O} 3$

7.946E-O 1

2. $502 \mathrm{E}+01$

$3.022 \mathrm{E}+\mathrm{OO}$
$2.744 \mathrm{E}+\mathrm{O} 2$

2.744E +O2

$-1.944 E+02$

$-2.112 E+00$

1. $189 E+00$

9. $505 E+00$

2. $694 \mathrm{E} \div \mathrm{O} 2$

8. $125 \mathrm{E}+01$

$-2.425 E+01$

7. $893 \mathrm{E}-01$

4. $361 \mathrm{E}+\mathrm{OO}$

7. 53 Y E - O

7. 53 Y $E-O 1$

4. $363 E+00$

$2.787 E+00$
$9.789 E-01$

3. $247 E+O 1$
3.0

91.5
3.8

8. 1

8.9
22.1

24.0

100.0

3.6
100.8

100.8
100.3

55.6

55.6
93.1

93.1
18.7

8.1

2.8
78.7

11.0

19.5

91.2

13. 1

5.0

6.6
9.7

19.6

100.2

100.1

4.5

16.7

38.8
101.4

6.1
71.4

65.5

31.2

5.9
16.1

4.3

16.

$+6.5$

7.3

6.8

5.2

10.6

100.8
3.2
1. $362 E+03$

2. $929 t: 03$

1. $682 \mathrm{E}+\mathrm{OA}$

2. $597 \mathrm{E}+\mathrm{O} 2$

9. $585 E+03$

$6.979 E+02$

$1.883 E+0$

5. $353 E+O 2$

2. $31 \mathrm{OE}+\mathrm{O} 2$

-3. $382 \mathrm{E}+\mathrm{O} 2$

$-1.600 E-O$

-2. $248 E+O$

2. $772 \mathrm{E}+02$

2. $641 \mathrm{E}+\mathrm{OO}$

1. $273 E+O O$

$1.091 \mathrm{E}+\mathrm{O}$

5. $478 E+O O$

$5.538 E+O O$

$8.644 E+O O$

7. 229E-O

1. 192E+O1

1. $517 \mathrm{E}+\mathrm{OO}$

1. $925 E+00$

3. $113 \mathrm{E}-01$

2. $744 \mathrm{E}+\mathrm{OO}$

1. $026 \mathrm{E}-02$

$3.579 \mathrm{E}+\mathrm{OO}$

1. $049 E+O$

1. $606 \mathrm{E}+04$

$5.438 E+O O$

5. $398 E+01$

1. $880 E+01$

$-1.574 \mathrm{E}+02$

$-1.729 E+O O$

$-5.787 E-D 1$

1. $718 \mathrm{E}+\mathrm{OO}$

2. $068 E+02$

$2.541 E+O 1$
$-9.858 E+O O$

4. 496E-O

$-3.28 B E-01$

2. $797 \mathrm{E}+00$

4. $037 E-01$

1. $973 E+00$

$-4.628 E-01$

$-6.760 E-01$
$3.678 E+00$
4.8

26.8

6.7
12.3

97.4

16.8

94.8

3.5

100.8

100.3

56.4

93.2

24.4
19.4

2. 8

79.1
10.6

11.6

89.1

13.9

7.4

8.5
14.2

23.3

100.2

4.0
12.6

12.6
4.0

5. 8

18.3

64.6
29.0

71.5

65.7

80.5

13.2

6.5
76.9

15.0

39.6

6. 1

8.3
7.6

38.1

100.8
5.3
1. $874 \mathrm{E}+03$

3. $298 \mathrm{BE}+03$

2. $831 \mathrm{E}+\mathrm{O} 4$

$4.889 \mathrm{E}+02$

$-4.538 E+03$

1. $249 E+04$

1. $31 \mathrm{BE}+03$

4. $051 E+01$

6. $543 E+02$

3. $641 \mathrm{E}+02$

$3.071 E-01$

2. $353 E+01$

$-1.353 E+01$

3. $533 \mathrm{E}+\mathrm{OO}$

2. $251 \mathrm{E}+00$

- $1.28 \mathrm{E}+0$

3. $181 E+O O$

$5.042 \mathrm{E}+00$

$-6.163 E+O O$
$7.311 E-01$

$536 \mathrm{E}+\mathrm{O}$

$.602 E+O O$

3. $060 E+00$

4. $816 E-01$

3. $876 \mathrm{E}+00$

$-9.279 E-03$

1. $320 E+01$

2. $673 \mathrm{E}+\mathrm{OO}$

9. $926 \mathrm{E}+0$

3. $616 \mathrm{E}+00$

2. $476 \mathrm{E}+0$

1. $898 E+02$

1. $898 E+02$

$7.811 \mathrm{E}-0$

1. $471 \mathrm{E}+00$

1. $941 E+O 2$

1. $173 E+01$

5. 462E-O 1

4. $932 E-O 1$

3. $431 E+O O$

$5.423 \mathrm{E}-01$

2. $892 E+O O$

$2.892 E+O O$

5. $760 E-O$

$-8.023 E-01$
$8.555 E+00$
4.4

24.6

5.4
10.2

97.2

14.1

18.2

3.5

100.8

100.3

23.8

93.3

O०. 1

2.8

79.6

12.9

90.7

16.3

4.5

4.9

7.1

16. 4

100.2

3.9

9. 9

4.6

12.2

21.9

21.0

64.9

75.1

14.7

55.2

77.3

13.2

27.4

6. 4

5. 8

24.6

100.8
3.7 
Na- 24

$\mathrm{Mg}-27$

A) - 28

$\mathrm{Cl}-38$

$\mathrm{K}-3 \mathrm{4}$
$\mathrm{Ca}-49$

Ti- 51

$V-52$
$M n-56$

$\mathrm{Cu}-66$

Sr- 87

In- 116

I -128

Dy -165

$u-235$

$\mathrm{Ga}-72$

As- 76

Br- 82

Sb- 122

$\mathrm{La}-14 \mathrm{O}$
$\mathrm{Sm}-153$

Yo- 175

Lu-177

$w-187$
$A u-198$

SC- 46

Cr- 51

$\mathrm{Fe}-59$

Zn- 65

$\mathrm{Se}-75$

$\mathrm{Rb}-\mathrm{B6}$

$2 r-9$

$\mathrm{Ag}-110$

Sb- 124

Ba-13!

Ce-14:

$\mathrm{Nd}-147$

$\mathrm{Eu}-152$

Tb- 160

$\mathrm{Yb}-169$

Lu-177

Hf -18

Ta-182

$\mathrm{Hg}-203$

$T h-233$

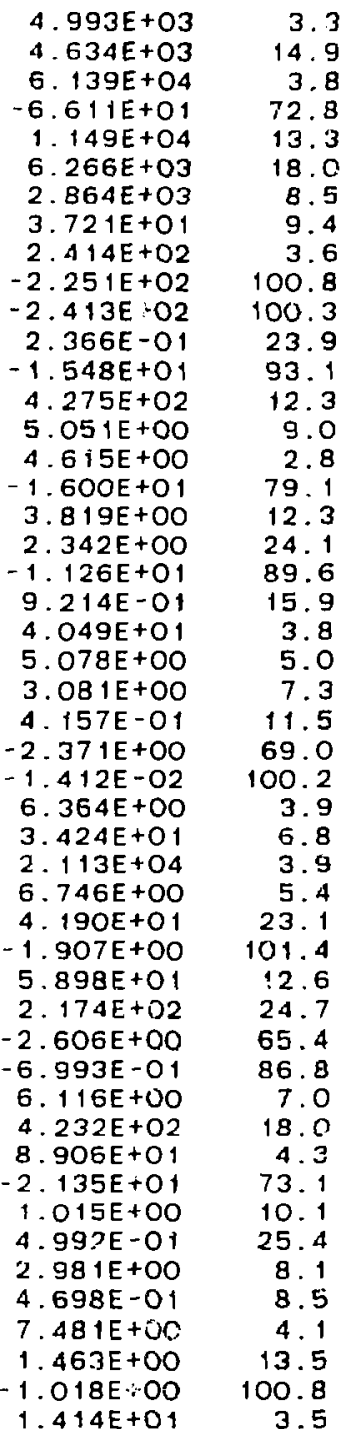

\begin{tabular}{|c|c|}
\hline $4.703 E+03$ & 3.5 \\
\hline $6.455 E+03$ & 13.4 \\
\hline $8.034 E+04$ & 3.7 \\
\hline 7. $570 E+01$ & 73.2 \\
\hline 1. $391 E+04$ & 16.2 \\
\hline $9227 E+03$ & 16.7 \\
\hline A. $236 E+O 3$ & 7.6 \\
\hline $6.450 E+01$ & 7.7 \\
\hline $2.991 E+02$ & 3.6 \\
\hline $2.685 \mathrm{E}+02$ & 100.8 \\
\hline $2.853 E+O 2$ & 100.3 \\
\hline $1.719 E-01$ & 55.4 \\
\hline $1.870 E+01$ & 93.2 \\
\hline 1. $363 E+02$ & 100.1 \\
\hline $3.252 E+00$ & 11.4 \\
\hline $4.273 E+00$ & 2.8 \\
\hline $1.831 E+01$ & 79.3 \\
\hline $3.661 E+00$ & i5. 1 \\
\hline $1.923 E+00$ & 47.6 \\
\hline $1.249 E+01$ & 89.5 \\
\hline $8.557 \mathrm{E}-01$ & 18.7 \\
\hline 3. $564 \mathrm{E}+01$ & 4.0 \\
\hline 4. $385 E+00$ & 5.0 \\
\hline $2.396 E+O O$ & 8.9 \\
\hline 4. $289 E-01$ & 12.5 \\
\hline $2.522 \mathrm{E}+00$ & 53.8 \\
\hline $1.559 \mathrm{E}-02$ & 100.2 \\
\hline 1. $.059 E+01$ & 3.8 \\
\hline $3.837 E+01$ & 6.3 \\
\hline $2.87 B E+04$ & 3.8 \\
\hline $1.033 E+01$ & 4.7 \\
\hline $1.81 O E+O 2$ & 10.5 \\
\hline 3. $166 \mathrm{E}+00$ & 101.4 \\
\hline $1.096 E+02$ & 9.1 \\
\hline $2.546 E+O 2$ & 71.5 \\
\hline $2.893 E+00$ & 65.2 \\
\hline $9.007 E-01$ & 80.6 \\
\hline 1. $138 E+01$ & 5.5 \\
\hline 4. $185 E+02$ & 22.6 \\
\hline $7.638 \mathrm{E}+01$ & 3.9 \\
\hline $3.389 E+01$ & 27.4 \\
\hline $8.947 E-01$ & 10.0 \\
\hline $7.070 E-01$ & 17.4 \\
\hline $3.295 E+00$ & 7.3 \\
\hline $4.723 E-01$ & 7.9 \\
\hline $6.517 E+00$ & 4.2 \\
\hline $1.485 E+00$ & 14.5 \\
\hline 1. $065 E+00$ & 100.8 \\
\hline $17+1 F+01$ & \\
\hline
\end{tabular}

2. $413 \mathrm{E}+\mathrm{O}$

1. $988 E+04$

2. $374 E+O 2$

$-4.434 \mathrm{E}+\mathrm{O} 3$

1. $667 \mathrm{E}+04$

1. $516 E+03$

3. $624 E+O 1$

6. $411 \mathrm{E}+\mathrm{O} 2$

2. $47 \mathrm{OE}+\mathrm{O}$

$-2.470 E+02$

- 1. $732 E-01$

$-2.355 E+01$

$-1.412 E+O 2$

3. $1533 E+O 0$

2. $315 \mathrm{E}+\mathrm{OO}$

-9. B85E+OO

4. $166 \mathrm{E}+\mathrm{OO}$

$5.026 E+O 0$

$-5.993 \mathrm{E}+\mathrm{O}$

$5.644 E-O$

$570 E+00$

2. $048 E+O O$

2. $639 \mathrm{E}+00$

4. $941 E-01$

3. $490 \mathrm{E}+\mathrm{OO}$

8. $411 \mathrm{E}-\mathrm{O}$

$5.230 E+O$

1. $304 E+3$

$6.472 \mathrm{E}+.23$

1. $750 \mathrm{OE} O$

4. $767 \mathrm{E}$ OO

3. $819 E+O O$

1. $538 \mathrm{E}+\mathrm{O}$

$-2.315 E+02$

$2.600 E+00$

7 856E-O

-

2. $408 E+02$

2. $008 E+O$

6. $948 E-O 1$

3. $172 \mathrm{E}+O O$

4. 506E-O

2. $2.23 \mathrm{E}+00$

$6.068 E-01$

$-1.010 E+O 0$
30. 4

14.3

97.3

13.5

11.2

3. 5

100.8

100. 3

93.3

100. 1

2. 8

79.9

10.7

11.9

11.9
90.2

90.2

5.2

5.3

6. 3

7.5

15.9
100.2

3.9

15. 1

4.9
10.8

100.4

26.7

100.0

65.0

65.0

63.6

58.2 6.1
72.8

72.8

20.2

22.1

B. 9

8.2

33.4
100.8

4.6 
$\mathrm{Na}-24$

$\begin{array}{ll}\mathrm{Mg}- & 24 \\ \mathrm{Mg} & \end{array}$

A1- 28

$\mathrm{Cl}-38$

$\mathrm{K}-42$
$\mathrm{Ca}-49$

$\mathrm{Ca}-49$
$\mathrm{~T}\}-51$

$v-52$

$\mathrm{Mn}-56$

Cu- 66

$\mathrm{Sr}-87$

In -116
I -128

Ba-139

Dy -165

$\mathrm{D}-235$
$\mathrm{G}-2$

Ga- :2

$\mathrm{Br}-\mathrm{B2}$

Mo- 99

Sb- 122

La-140

$\mathrm{sm}-153$

Yb- 175

$Y U-177$
$W-187$

$W-187$
$\mathrm{Au}-198$

Sc- 46

$\mathrm{Cr}-51$

$\mathrm{CO}-60$

$2 n-65$

Se- 75

$R b-86$
$Z r-95$

$\mathrm{Ag}-110$

$5 b-124$

cs -134

Ba-131

$\mathrm{Ce}-141$

Nd- 147

Eu-152

$T b-160$

$\mathrm{Yb}-169$
$\mathrm{Lu}-177$

HF -181

$\mathrm{T} a-182$

N $\begin{array}{r}\mathrm{Hg}-203 \\ \mathrm{Th}-233\end{array}$

\begin{tabular}{|c|c|}
\hline $4.702 E+03$ & 3.4 \\
\hline $3.884 E+03$ & 17.1 \\
\hline $6.147 E+04$ & 4.0 \\
\hline$-7.14 O E+01$ & 73.0 \\
\hline $1.081 E+04$ & 14.4 \\
\hline $1.001 E+04$ & 15.2 \\
\hline 3. $236 E+03$ & 8.4 \\
\hline $4.582 E+01$ & 8.4 \\
\hline $2.688 E+O 2$ & 3.6 \\
\hline$-2.433 \varepsilon+02$ & 100.8 \\
\hline$-2.67 O E+O 2$ & 100.3 \\
\hline $2.020 E-01$ & 25. \\
\hline$-1.726 E+01$ & \\
\hline $4.277 E+02$ & 12.7 \\
\hline $5.588 E+C$ & 9. \\
\hline $4.675 E+00$ & \\
\hline$-1.897 E+01$ & 79.2 \\
\hline $2.11 B E+O 1$ & 8.2 \\
\hline$-1.959 E+00$ & 47.5 \\
\hline$-1.318 E+01$ & 89.2 \\
\hline $1.718 E+O O$ & 11.7 \\
\hline 5. $136 E+C$ & 3.8 \\
\hline $6.767 E+O O$ & 4.7 \\
\hline 3.81 & 6.9 \\
\hline 6.02 & 8.2 \\
\hline $1.726 E+\infty O$ & 31.0 \\
\hline$-1.607 E-02$ & 100.2 \\
\hline $7.778 E+00$ & 3.5 \\
\hline $2.856 E+01$ & 7.5 \\
\hline 3.2 & 3.8 \\
\hline 1.1 & 4.7 \\
\hline 1. $104 E+02$ & 13.4 \\
\hline$-6.232 E+00$ & $82 . c$ \\
\hline 6.45 & 12. \\
\hline $3.216 \mathrm{E}+\mathrm{O} 2$ & 25.4 \\
\hline$-2.289 E+00$ & 65.4 \\
\hline $1.413 E+00$ & 28.9 \\
\hline $6.426 E+\infty 0$ & 7.0 \\
\hline $4.858 E+02$ & 14.3 \\
\hline $1.147 E+O 2$ & 4.1 \\
\hline $3.838 E+01$ & 25.5 \\
\hline 1. $343 E+O O$ & 8.7 \\
\hline $2.057 E+00$ & 13.8 \\
\hline $3.171 \mathrm{E}+00$ & 10.7 \\
\hline $6.779 E-01$ & j. B \\
\hline $6.621 \mathrm{E}+\infty 0$ & 4.4 \\
\hline $1.434 E+O O$ & 13.7 \\
\hline$-8.841 E-01$ & 100.8 \\
\hline $1.661 E+01$ & 3.4 \\
\hline
\end{tabular}

\begin{tabular}{|c|c|}
\hline 2. $148 E+O 3$ & 4.1 \\
\hline 4. $166 E+O 3$ & 16.4 \\
\hline $6.449 E+O 4$ & 4.0 \\
\hline$-6.072 \mathrm{E}+01$ & 74.6 \\
\hline$-3.943 E+03$ & 96.8 \\
\hline $1.393 E+04$ & 13.1 \\
\hline $2.864 E+03$ & 9.0 \\
\hline $6.4+7 E+01$ & 8.4 \\
\hline $3.729 E+O 2$ & 3.5 \\
\hline$-2.208 \mathrm{E}+02$ & 100.8 \\
\hline$-2.758 \mathrm{E}+02$ & 100.3 \\
\hline $2.321 E-01$ & 15.4 \\
\hline$-1.820 E+01$ & 93.2 \\
\hline $1.651 E+02$ & 21.2 \\
\hline $4.574 E+O O$ & 8.6 \\
\hline 4. $385 E+00$ & 2.8 \\
\hline$-1.272 E+01$ & 79.6 \\
\hline $3.972 E+00$ & 11.7 \\
\hline $2.845 E+00$ & 14.5 \\
\hline$-6.881 E+00$ & 90.8 \\
\hline $7.483 \mathrm{E}-01$ & 14.7 \\
\hline $2.363 \mathrm{E}+01$ & 4. 1 \\
\hline $3.760 E+00$ & 4.9 \\
\hline $3.535 E+\infty 0$ & 5.6 \\
\hline $5.989 E-01$ & 7.2 \\
\hline $2.619 E+00$ & 23.0 \\
\hline$-1.042 \mathrm{E}-02$ & 100.2 \\
\hline $9.162 \mathrm{E}+00$ & 3.9 \\
\hline $2.381 E+01$ & 7.0 \\
\hline $1.881 E+04$ & 3.9 \\
\hline $4.476 \mathrm{E}+00$ & 5.9 \\
\hline $1.645 E+02$ & 10.7 \\
\hline$-2.357 E+O O$ & 72.2 \\
\hline $4.073 E+01$ & 12.9 \\
\hline$-2.290 \mathrm{E}+\mathrm{O} 2$ & 71.6 \\
\hline$-2.549 E+00$ & 65.1 \\
\hline 1. $085 E+00$ & 31.7 \\
\hline $6.784 E+00$ & 6.4 \\
\hline$-2.349 E+02$ & 54.5 \\
\hline $4.749 E+01$ & 4.1 \\
\hline$-1.347 E+01$ & 77.9 \\
\hline $5.966 \mathrm{E}-01$ & 12.8 \\
\hline $5.884 E-01$ & 25.9 \\
\hline $3.246 E+0 O$ & 6.6 \\
\hline $5.649 E-01$ & 6.8 \\
\hline $5.253 E+00$ & 4.5 \\
\hline 1. $407 E+00$ & 13.3 \\
\hline$-9.559 E-01$ & 100.8 \\
\hline $1.387 \mathrm{E}+01$ & 3.4 \\
\hline
\end{tabular}

$\begin{array}{rr}2.587 E+O 3 & 3.9 \\ 3.22 O E+O 3 & 17.8 \\ 8.518 E+O 4 & 3.7 \\ -6.003 E+O 1 & 74.8 \\ 8.457 E+O 3 & 19.8 \\ 8.087 E+O 3 & 16.8 \\ 3.17 O E+O 3 & 8.0 \\ 5.7 O 3 E+O 1 & 7.8 \\ 2.228 E+O 2 & 3.6 \\ -2.234 E+O 2 & 100.8 \\ -2.457 E+O 2 & 100.3 \\ -1.465 E-O 1 & 55.4 \\ -1.586 E+O 1 & 93.2 \\ -1.163 E+O 2 & 100.1 \\ 6.278 E+O O & 8.6 \\ 4.723 E+O O & 2.8 \\ 1.619 E+O 1 & 80.2 \\ 4.32 O E+O O & 14.3 \\ 1.806 E+O O & 23.2 \\ 1.219 E+O 1 & 89.1 \\ 1.119 E+O O & 16.7 \\ 5.463 E+O 1 & 3.8 \\ 6.767 E+O O & 4.7 \\ 4.237 E+O O & 6.2 \\ 6.939 E-O 1 & 7.1 \\ 1.846 E+O O & 30.5 \\ -1.43 O E-O 2 & 100.2 \\ 8.462 E+O O & 3.9 \\ 2.775 E+O 1 & 8.5 \\ 2.310 E+O 4 & 4.0 \\ 6.336 E+O O & 5.7 \\ 7.975 E+O 1 & 16.8 \\ 4.501 E+O O & 101.4 \\ 6.323 E+O 1 & 14.5 \\ 2.335 E+O 2 & 99.0 \\ 3.203 E+O O & 65.3 \\ 9.006 E-O 1 & 86.4 \\ 1.072 E+O 1 & 6.1 \\ 3.211 E+O 2 & 56.2 \\ 1.134 E+O 2 & 4.2 \\ 2.551 E+O 1 & 73.4 \\ 1.17 O E+O O & 9.6 \\ 1.097 E+O O & 17.3 \\ 4.7 O 9 E+O O & 7.4 \\ 7.741 E-O 1 & 7.4 \\ 7.689 E+O O & 4.5 \\ 1.629 E+O O & 14.2 \\ 1.234 E+O O & 100.8 \\ 1.547 E+O 1 & 3.6\end{array}$

1. $475 \mathrm{E}+\mathrm{O}$

3. $283 \mathrm{E}+03$

2. $525 \mathrm{E}+04$

4. $848 E+01$

1. $263 \mathrm{E}+04$

. $250 E+03$

. $804 E+01$

3. $796 \mathrm{E}+\mathrm{O} 2$

$-2.718 E+02$

$-1.763 E+01$

$-1.103 E+02$

3. 359 E rOO

2. $904 \mathrm{E}+0 \mathrm{O}$

$1.067 \mathrm{E}+01$

4. $090 E+00$

$3.409 E+00$

$6.082 E+00$

1. $926 \mathrm{E}+01$

$3.033 E+00$

. $015 E+00$

3. $664 \mathrm{E}-01$

1. $327 E+00$

8. $762 \mathrm{E}-03$

$4.468 E+D O$

1. $449 E+01$

. $267 E+03$

3. $425 E+00$

. $824 E+01$

3. $825 E+00$

. $924 E+01$

-1.50OE+02

1. $739 \mathrm{E}+\mathrm{OO}$

5. 876E-O1

1. $750 E+O O$

1. $864 E+O 2$

1. $027 E+0$

$5.336 \mathrm{E}-0$

4. 536E-O

2. $054 E+00$

3. $716 \mathrm{E}-\mathrm{O}$

2. $746 \mathrm{E}+O C$

3. $386 \mathrm{E}-01$

$5.973 E+O O$
4.6

5.5
76.9

97.2

14.6

13.5

12.0

100.8

100.8

56.1

93.3

100.1

9.4
2.8

79.8

10.9

12.8

90.5

4. 3

5.0

7.0

10.2

26.5

9.5

4.5

6.9
15.9

101.4

18. 5

71.5

65.1

12.0

50.4

4.6

76.7

14.6

21.6

9.3

6.2
29.4

29.4
100.8

4.3 


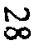

$\begin{array}{lc}\text { FILE ID } & 6100650 \\ \text { SAMPLE } & 021 \\ \text { DUPLICATE } & 042 \\ \text { CLUSTER } & 10 \\ \text { TYPE } & L\end{array}$

6100660

022

10

6100670
023
057
10
1

6100680

024

09

\section{ELEMENT}

\begin{tabular}{|c|c|c|}
\hline $\mathrm{Na}-24$ & $3.239 E+03$ & 3.7 \\
\hline$M g-27$ & $-1.863 E+03$ & 93.7 \\
\hline$A \overrightarrow{1}-28$ & $4.706 E+04$ & 4.4 \\
\hline$C 1-38$ & $-6.819 E+01$ & 74. \\
\hline$k-42$ & $6.995 E+03$ & 22.8 \\
\hline $\mathrm{Ca}-49$ & 1. $290 E+04$ & \\
\hline$T i-51$ & $1.523 E+03$ & \\
\hline$v-52$ & $2.751 E+01$ & \\
\hline$M_{17}-56$ & $3.584 \mathrm{E}+02$ & 3.6 \\
\hline Cu- 66 & $-2.400 E+02$ & 100.8 \\
\hline$S r-87$ & $-2.949 E+02$ & 100. \\
\hline In -116 & $-1.546 E-01$ & 56. \\
\hline$I-128$ & $-1.926 E+01$ & 93. \\
\hline $8 a-139$ & $-1.33 B E+O 2$ & 100. \\
\hline$D_{Y}-165$ & 4. $120 E+00$ & 10. \\
\hline$U-235$ & $5.462 E+0 O$ & 2. \\
\hline Ga- 72 & $-1.596 E+01$ & 79. \\
\hline As - 76 & $3.759 E+00$ & 12. \\
\hline $\mathrm{Br}-82$ & $3.566 E+00$ & 18. \\
\hline Mo- 99 & $-1.067 E+01$ & 89. \\
\hline Sb- 122 & 7. $055 E-01$ & 19. \\
\hline$L a-140$ & $2.451 E+01$ & \\
\hline $5 m-153$ & $3.229 E+O O$ & 5. \\
\hline$Y b-175$ & 2. $352 \mathrm{E}+00$ & B. 4 \\
\hline Lu -177 & 4. $459 E-01$ & 12.3 \\
\hline$W-187$ & $3.595 E+00$ & 21.2 \\
\hline$A U-198$ & $-1.287 E-02$ & 100.2 \\
\hline $5 c-46$ & $5.251 E+O O$ & 3.9 \\
\hline $\mathrm{Cr}-51$ & $1.271 E+01$ & 10.3 \\
\hline $\mathrm{Fe}-59$ & $6.600 E+03$ & 4.5 \\
\hline Co- 60 & 1. $720 E+O O$ & 8.7 \\
\hline $2 n-65$ & 1. $134 E+02$ & 11.3 \\
\hline $5 e-75$ & $3.430 E+O O$ & 37.6 \\
\hline$R b-86$ & $2.553 E+01$ & 18.8 \\
\hline $2 r-95$ & 1. $163 \mathrm{E}+\mathrm{O} 2$ & 27.1 \\
\hline$A g-110$ & $-2.066 \mathrm{E}+00$ & 65.2 \\
\hline $5 b-124$ & $-7.010 E-01$ & 81.7 \\
\hline$C s-134$ & $2.001 E+00$ & 12.2 \\
\hline $8 a-131$ & $1.460 E+02$ & 22.9 \\
\hline $\mathrm{Ce}-141$ & $5.408 E+01$ & 4.2 \\
\hline$N d-147$ & $-1.272 E+01$ & 75.7 \\
\hline$E u-152$ & $5.503 E-01$ & 13.2 \\
\hline TO -160 & $4.447 E-01$ & 23.9 \\
\hline$Y b-169$ & 3. $329 E+00$ & 6.1 \\
\hline$L u-177$ & $4.655 E-01$ & 7.5 \\
\hline$H F-181$ & $5.651 \mathrm{E}+00$ & 4.4 \\
\hline$T a-182$ & 1. $365 E+00$ & 13.5 \\
\hline $\mathrm{Hg}-203$ & $-7.958 E-01$ & 100.8 \\
\hline$T h-233$ & $2.277 E+01$ & 3.2 \\
\hline
\end{tabular}

\begin{tabular}{|c|c|}
\hline $3.761 E+03$ & 3.6 \\
\hline$-1.792 E+03$ & 93.4 \\
\hline $3.088 E+04$ & 5.0 \\
\hline 9. $759 E+O 1$ & 25.8 \\
\hline 4. $250 E+03$ & 29.4 \\
\hline 1. $353 E+O 4$ & 14.5 \\
\hline 1. $589 E+03$ & 14.8 \\
\hline 1. $825 E+01$ & 14.5 \\
\hline 4. $\triangle O O E+O 2$ & 3.5 \\
\hline$-2.396 E+O 2$ & 100.8 \\
\hline$-2.967 E+02$ & 100.3 \\
\hline$-1.573 E-01$ & 56.2 \\
\hline$-1.965 E+01$ & 93.2 \\
\hline 2. $143 E+O 2$ & 20.1 \\
\hline $2.73 B E+00$ & 10.8 \\
\hline $4.968 E+00$ & 2.8 \\
\hline$-1.417 \mathrm{E}+01$ & 78.8 \\
\hline $7.046 E+00$ & 9.2 \\
\hline $6.425 E+00$ & 11.7 \\
\hline$-7.052 E+00$ & 90.7 \\
\hline 1. $123 E+O O$ & 11.1 \\
\hline $1.734 \mathrm{E}+01$ & 4.3 \\
\hline $2.7 \mathrm{BOE}+\mathrm{OO}$ & 5.1 \\
\hline $2.040 E+00$ & 7.9 \\
\hline $3.684 E-01$ & 11.5 \\
\hline $4.192 E+00$ & 16.5 \\
\hline$-1.068 \mathrm{E}-02$ & 100.2 \\
\hline $3.967 \mathrm{E}+00$ & 4.0 \\
\hline $9.069 E+00$ & 18.8 \\
\hline $6.106 E+03$ & 4.8 \\
\hline $6.219 E-01$ & 19.9 \\
\hline$-6.34 B E+00$ & 100.4 \\
\hline$-3.019 E+00$ & 58.1 \\
\hline $2.749 E+01$ & 18.9 \\
\hline$-1.391 E+O 2$ & 92.2 \\
\hline$-2.161 E+00$ & 65.1 \\
\hline 8. $960 E-01$ & 32.0 \\
\hline $1.611 E+O O$ & 18.0 \\
\hline$-1.979 E+02$ & 49.0 \\
\hline $3.717 E+01$ & 4. 8 \\
\hline$-1.98 \backslash E+01$ & 71.7 \\
\hline 3. $489 E-01$ & 20.5 \\
\hline $6.527 \mathrm{E}-01$ & 28.0 \\
\hline $2.039 E+00$ & 13.5 \\
\hline $3.684 E-01$ & 10.2 \\
\hline 3. $313 E+O O$ & 5.7 \\
\hline $1.231 \mathrm{E}+00$ & 17.5 \\
\hline$-8.968 E-01$ & 100.8 \\
\hline $1.457 E+01$ & \\
\hline
\end{tabular}

\begin{tabular}{|c|c|}
\hline 9. $104 E+03$ & 4.8 \\
\hline $2.648 \mathrm{E}+03$ & 21.2 \\
\hline $2.042 E+04$ & 5.7 \\
\hline 1. $364 \mathrm{AE}+\mathrm{O} 2$ & 15.1 \\
\hline $3.082 E+03$ & 97.2 \\
\hline 1. $216 E+O^{4}$ & \\
\hline $1.314 E+03$ & 17.5 \\
\hline 1. $660 E+01$ & 16.4 \\
\hline 3. $154 E+O 2$ & 3.6 \\
\hline 1. $785 \mathrm{E}+02$ & 100.8 \\
\hline 2. $476 E+02$ & 100.3 \\
\hline f. $182 E-01$ & 56.4 \\
\hline $1.639 E+01$ & 93.2 \\
\hline $3.217 E+02$ & 14.5 \\
\hline i. $725 E+00$ & 18.1 \\
\hline $1.882 \mathrm{E}+00$ & 2.8 \\
\hline $1.028 \mathrm{E}+01$ & 79.9 \\
\hline $3.298 E+00$ & 14.0 \\
\hline $7.451 E+00$ & 10.8 \\
\hline $7.363 E+00$ & 89.0 \\
\hline 2. $147 E-01$ & 100.1 \\
\hline $9.3775+00$ & 5.1 \\
\hline 1. $33 B E+\infty O$ & 5.3 \\
\hline $1.514 \mathrm{E}+00$ & 8.9 \\
\hline $2.253 \mathrm{E}-01$ & 13.5 \\
\hline 2. $42 O E+O O$ & 19.5 \\
\hline $8.716 \mathrm{E}-03$ & 100.2 \\
\hline 2. $909 E+00$ & 4.0 \\
\hline $9.591 E+00$ & 11.7 \\
\hline $4.574 E+03$ & 4.8 \\
\hline $1.053 E+00$ & 11.8 \\
\hline 2. $339 E+01$ & 28.3 \\
\hline $2.808 E+00$ & 26.2 \\
\hline $2.613 E+01$ & 18.9 \\
\hline 1. $172 \mathrm{E}+\mathrm{O} 2$ & 71.6 \\
\hline $1.353 E+\infty O$ & 65.2 \\
\hline $5.064 \mathrm{E}-04$ & 81.8 \\
\hline 1. $169 \mathrm{E}+00$ & 14.8 \\
\hline $3.492 \mathrm{E}+\mathrm{O} 2$ & 13.4 \\
\hline 2. $177 \mathrm{E}+01$ & 5.3 \\
\hline 8. $349 E+00$ & 75.9 \\
\hline $2.827 \mathrm{E}-01$ & 20.3 \\
\hline $2.944 \mathrm{E}-01$ & 21.7 \\
\hline $1.816 E+00$ & B. 9 \\
\hline $1.920 E-01$ & 11.3 \\
\hline $2.434 \mathrm{E}+\mathrm{OO}$ & 6.0 \\
\hline $3.878 E-01$ & 21.4 \\
\hline 5. $419 E-01$ & 100.8 \\
\hline $733 E+00$ & \\
\hline
\end{tabular}

$2.888 E+O 3$

$4.643 \mathrm{E}+\mathrm{O}$

$7.064 E+04$

5. $560 E+O 1$

$7.643 E+03$

9. $601 E+03$

2. $73 \dot{b} \mathrm{O}+\mathrm{O}$

9. $659 \mathrm{E}+\mathrm{O} 1$

2. $368 E+02$

1. $924 E+02$

1. 594E-01

1. $346 E+O 1$

8. $648 E+01$

6. $429 E+O O$

5. $325 \mathrm{E}+00$

2. $165 E+01$

5. $085 \mathrm{E}+00$

1. $303 E+00$

- 1. $704 E+00$

2. $358 E+00$

4. $030 E+01$

6. $619 E+00$

4. $345 E+00$

7. $500 E-O 1$

3. $239 \mathrm{E}+\mathrm{OO}$

1. $025 E-02$

9. $365 \mathrm{E}+00$

3. $286 \mathrm{E}+01$

2. $418 E+O 4$

2. $100 E+01$

3. $992 \mathrm{E}+02$

- $3.756 E+O D$

7. $325 \mathrm{E}+\mathrm{O} 1$

3. $246 \mathrm{E}+\mathrm{O} 2$

1. $847 \mathrm{E}+00$

2. $375 E+\infty O$

$9.209 E+0 O$

-2. $099 \mathrm{E}+\mathrm{CI}$

$-2.099 E+C 12$
9. $376 E+01$

5 OBOE+O1

1. $160 \mathrm{O}+0$

1. $160 E+00$

5. $189 E+O O$

5. $029 E-01$

8. $029 E-01$

5. BO4E +OO

1. $504 E+00$

1. $454 \mathrm{E}+01$
3.5

100.8

100.3

24.7

93.3

100. 1

2. 8

22.9

10.4

47.4

100.0

3.7

4. 8

5.0

6.0

100.2 


$\begin{array}{lc}\text { FILE ID } & 6100690 \\ \text { SAMPLE } & 025 \\ \text { DUPLICATE } & 036 \\ \text { CLUSTER } & 10 \\ \text { TYPE } & \text { L }\end{array}$

6100700
6100710
6100720

028

04

06

\section{ELEMENT}

$\mathrm{Na}-24$

$\mathrm{Mg}^{-} 27$

A) - 28

Cl- 38

$K-42$

Ti- 51

$v-52$

$\mathrm{Mn}-56$

Cu- 66

Sr- 87
In- 116

I -128

Ba-139

$D y-165$
$y-235$

$\mathrm{Ga}-72$

As - 76

$\mathrm{Br}-82$

Mo- 99

Sb- 122

La-140

Sm- 153

Yu- 177

Lu- 177

Au- 198

Sc- 46

$\mathrm{Cr}-51$
$\mathrm{Fe}-59$

$\mathrm{Co}-60$

$\mathrm{Zn}-65$

Se- 75

$\mathrm{Rb}-86$

$2 r-95$

$\mathrm{Ag}-110$

Sb- 124

Cs -134

Ba-13i

$\mathrm{Ce}-141$

Eu-152

$\mathrm{Yb}-169$

Lu- 177

$\mathrm{Hf}-181$

Ta-182

$\mathrm{Hg}-203$

\begin{tabular}{|c|c|}
\hline 1. $118 \mathrm{E}+03$ & 4.8 \\
\hline $2.736 E+03$ & 24.1 \\
\hline $4.491 E+04$ & 4. 2 \\
\hline 9. $165 E+01$ & 22.8 \\
\hline$-3.492 E+03$ & 97.2 \\
\hline $1.054 E+04$ & 13.7 \\
\hline $1.865 E+03$ & 12.9 \\
\hline 3. $123 \mathrm{E}+01$ & 9.3 \\
\hline 4. $590 \mathrm{E}+\mathrm{O} 2$ & 3.5 \\
\hline$-1.955 E+02$ & 100.8 \\
\hline$-2.829 E+O 2$ & 100.3 \\
\hline $1.409 E-01$ & 29.3 \\
\hline$-9.835 E+01$ & 93.2 \\
\hline $1.686 E+02$ & 26.1 \\
\hline 3. $254 E+00$ & 11.6 \\
\hline $4.023 E+00$ & 2.8 \\
\hline$-1.15 O E+01$ & 80.3 \\
\hline $4.557 E+00$ & 10.8 \\
\hline $3.800 E+00$ & 12.4 \\
\hline$-3.149 E+O O$ & 100.0 \\
\hline $9.219 E-01$ & 11.1 \\
\hline $2.078 E+01$ & 4.2 \\
\hline $2.981 E+00$ & 5.5 \\
\hline $2.376 E+00$ & 7.5 \\
\hline $3.294 E-01$ & 11.0 \\
\hline 1. $908 E+00$ & 22.8 \\
\hline$-9.315 E-03$ & 100.2 \\
\hline $4.922 E+00$ & 3.9 \\
\hline $1.428 E+01$ & 10.6 \\
\hline $8.078 E+03$ & 4.4 \\
\hline 2. $194 E+O O$ & 8.4 \\
\hline $3.997 E+01$ & 19.7 \\
\hline $3.816 E+00$ & 24.9 \\
\hline $1.945 E+01$ & 21.6 \\
\hline$-1.912 E+O 2$ & 71.6 \\
\hline$-2.159 E+O O$ & 65.0 \\
\hline$-6.114 \mathrm{E}-01$ & 83.9 \\
\hline $2.856 E+00$ & 9.9 \\
\hline $2.193 E+O 2$ & 19.9 \\
\hline $4.533 E+01$ & 4.5 \\
\hline$-1.784 E+01$ & 73.3 \\
\hline 4. $958 E-01$ & 14,8 \\
\hline $6.827 E-01$ & 22.0 \\
\hline $2.133 E+00$ & 12.0 \\
\hline $2.864 \mathrm{E}-01$ & $\begin{array}{r}10 \cdot 1 \\
5\end{array}$ \\
\hline $\begin{array}{l}3.305 E+00 \\
1.376 E+00\end{array}$ & $\begin{array}{r}5.7 \\
13.0\end{array}$ \\
\hline$-2.375 E-01$ & 100.8 \\
\hline $1.264 E+01$ & 3.0 \\
\hline
\end{tabular}

\begin{tabular}{|c|c|}
\hline $5.167 E+03$ & 3.3 \\
\hline$-1.906 E+03$ & 93.5 \\
\hline 3. $785 \mathrm{E}+04$ & 4.4 \\
\hline$-7.911 E+01$ & 72.7 \\
\hline $5.564 E+03$ & 19.2 \\
\hline $1.501 E+04$ & 12.7 \\
\hline 2. $122 \mathrm{E}$ & 10.2 \\
\hline $2.551 E+01$ & 12.8 \\
\hline 4. $363 E+O 2$ & 3.5 \\
\hline$-2.476 E+02$ & 100.8 \\
\hline$-2.941 E+02$ & 100.3 \\
\hline $2.875 E-01$ & 18.1 \\
\hline$-1.940 E+01$ & 93.2 \\
\hline $4.614 E+02$ & 12.4 \\
\hline $4.343 E+O O$ & 10.6 \\
\hline $5.769 E+00$ & 2.8 \\
\hline$-1.458 E+01$ & 78.9 \\
\hline $5.266 E+00$ & 11.4 \\
\hline $5.242 E+00$ & 12.6 \\
\hline$-1.123 E+O 1$ & 89.7 \\
\hline $8.892 E-01$ & 14.1 \\
\hline $2.349 E+01$ & 4. 1 \\
\hline 3. $514 \mathrm{E}+00$ & 5.3 \\
\hline $3.711 E+O O$ & 6.5 \\
\hline $7.242 E-01$ & 7.9 \\
\hline$-2.281 E+O O$ & 59.9 \\
\hline$-1.438 E-02$ & 100.2 \\
\hline $5.397 \mathrm{E}+00$ & 3.9 \\
\hline $1.293 E+01$ & 11.5 \\
\hline $1.862 E+O 3$ & 7.1 \\
\hline $7.377 \mathrm{E}-01$ & 17.2 \\
\hline $2.294 E+01$ & 32.8 \\
\hline$-2.883 E+00$ & 66.7 \\
\hline $3.848 E+01$ & 14.5 \\
\hline$-1.919 E+02$ & 71.7 \\
\hline$-2.242 E+00$ & 65.1 \\
\hline$-6.777 E-01$ & 83.9 \\
\hline $1.863 E+00$ & 14.4 \\
\hline $4.129 E+02$ & 11.5 \\
\hline $4.880 E+01$ & 4.4 \\
\hline$-1.826 E+01$ & 72.2 \\
\hline $4.520 E-01$ & 17.7 \\
\hline 4. 599E-01 & 25.9 \\
\hline $3.895 E+00$ & 7.1 \\
\hline $5.982 E-01$ & 6.7 \\
\hline $6.111 E+00$ & 4.5 \\
\hline 2. $367 E+O O$ & 11.1 \\
\hline$-9.053 E-01$ & 100.8 \\
\hline $2.231 E+01$ & 3.3 \\
\hline
\end{tabular}

6. $582 \mathrm{E}+\mathrm{O}$

3. $719 E+03$

$6.861 \mathrm{E}+\mathrm{O} 4$

6. $960 E+01$

8. $965 \mathrm{E}+03$

9. $022 E+03$

1. $897 E+O^{3}$

2. $252 E+O$

2. $265 E+02$

-2. $295 E+02$

$-2.310 \mathrm{E}$

1. $523 E-01$

$-1.499 E+01$

8. $322 E+O 2$

$6.046 E+00$

$8.969 E+00$

2. $354 \mathrm{E}+\mathrm{O}$

4. $395 E+O O$

1. $793 E+O 0$

$7.964 \mathrm{E}-\mathrm{O} 1$

8. $937 E-01$

3. $678 E+01$

$6.391 E+O O$

3. $782 \mathrm{E}+00$

$5.664 E-O$

2. $194 \mathrm{E}+\mathrm{O}$

1. $194 \mathrm{E}+00$

1. $229 E-O 2$

5. $270 \mathrm{E}+0 \mathrm{O}$

1. $126 \mathrm{E}+0$

$5.63 O E+O 3$

1. $340 E+O C$

7. $272 \mathrm{E}+\mathrm{O}$

$2.620 \mathrm{E}+\mathrm{OO}$

$6.734 E+O 1$

1. $904 \mathrm{E}+02$

$-1.704 E+O O$

$7.875 \mathrm{E}-\mathrm{O}$

$3.058 E+O$

8. $278 E+02$

7. $455 \mathrm{E}+01$

3. $13 E E+01$

6.835E-O

1. $043 \mathrm{E}+00$

4. $621 \mathrm{E}+O O$

5. $734 \mathrm{E}-01$

6. 58OE+OO

2. $609 E+00$

$-6.690 E-01$

3. $146 \mathrm{E}+01$
3. 1

25.3

72.3

14.9

9.9

2.4

100.8

100. 3

25.3

93.1

8. 4

2.8

17.5

10.6

21.8

11.4
11.5

3.7
5.4

5.7

9.4

25.7

100. 2

3.9
10.2

4. 4

8.7
12.3

77.9

8.2
19.9

65.0

28.0

7.6

3.9

19.6

10.1

5. 2

5. 3
3.7

8.4

100.8 


$\begin{array}{lc}\text { FILE ID } & 6100730 \\ \text { SAMPLE } & 029 \\ \text { DUPLICATE } & 043 \\ \text { CLUSTER } & 10 \\ \text { TYPE } & \mathrm{L}\end{array}$

6100750
031
005
09

og

\section{0 032 \\ 026
05}

L

\section{ELEMENT}

$\mathrm{Na}-24$

$\mathrm{Mg}^{-2} 27$

A) - 28

K - 42

Ca- 49

$T i-51$

$v-52$
$M n-56$

$\mathrm{Cu}-66$

$\mathrm{Sr}-87$

In-116

$1+128$
$B a-139$

Ba -139

$0 y-165$
$u-235$

$\mathrm{Ga}-72$

As - 76

Br- 82

Sb- 122

La- 140

$\mathrm{Sm}-153$

$Y b-175$

LU
$W-177$
$W-187$

$W-187$
$A U-198$

$\mathrm{SC}-46$

$\mathrm{Cr}-51$

$\mathrm{CO}-60$

Se- 75

$\mathrm{Rb}-\mathrm{B} 6$

$2 r-95$

Ag- 110

$S b-12 d$

Cs-134

Ba-131

Ce-141

$\mathrm{Nd}-147$

Eu- 152

Tb- 160

Yo- 169

LU- 177

Hf -181

Ta- 182

$\mathrm{Hg}-203$

\begin{tabular}{|c|c|}
\hline $\begin{array}{r}4.144 \mathrm{E}+03 \\
-1.646 \mathrm{E}+03 \\
3.404 \mathrm{E}+04\end{array}$ & $\begin{array}{r}3.4 \\
92.9 \\
4.5\end{array}$ \\
\hline $\begin{array}{r}3.404 E+04 \\
-6.373 E+01\end{array}$ & $\begin{array}{r}4.5 \\
73.3\end{array}$ \\
\hline $5.207 E+03$ & 26.2 \\
\hline 1. $286 \mathrm{E}+04$ & 12.1 \\
\hline $2.015 \mathrm{E}+03$ & 11.6 \\
\hline $2.541 E+O 1$ & 12.1 \\
\hline $3.479 \mathrm{E}+02$ & 3.5 \\
\hline$-2.097 E+02$ & 100.8 \\
\hline$-2.458 E+02$ & 100.3 \\
\hline$-1.363 E-01$ & 55.8 \\
\hline$-1.615 E+01$ & 93.2 \\
\hline 4. $119 E+02$ & 10.1 \\
\hline 3. $801 E+O O$ & 8.8 \\
\hline $4.929 E+00$ & 2.8 \\
\hline$-1.205 E+01$ & 79.0 \\
\hline $5.467 \mathrm{E}+00$ & 11.2 \\
\hline $5.556 E+00$ & 12.0 \\
\hline$-9.333 E+O O$ & 89.6 \\
\hline $9.066 \mathrm{E}-01$ & 13.2 \\
\hline $1.929 E+O 1$ & 4. 1 \\
\hline $2.604 E+00$ & 5.4 \\
\hline $2.255 E+00$ & 7.6 \\
\hline 3. $065 E-01$ & 13.0 \\
\hline $2.773 E+00$ & 20.0 \\
\hline$-1.193 \mathrm{E}-02$ & 100.2 \\
\hline $4.430 E+O O$ & 3.9 \\
\hline 8. $891 E+00$ & 13.4 \\
\hline $6.141 E+03$ & 4.6 \\
\hline 1. $246 E+\infty O$ & 11.0 \\
\hline $1.557 E+01$ & 43.7 \\
\hline$-2.906 E+00$ & 90.8 \\
\hline 2. $228 E+O T$ & $19 . B$ \\
\hline$-1.762 E+O 2$ & 71.5 \\
\hline$-1.990 E+0 O$ & 65.3 \\
\hline$-6.617 \mathrm{E}-01$ & 80.7 \\
\hline 2. $144 E+O O$ & 10.9 \\
\hline $2.780 \mathrm{O}+\mathrm{O} 2$ & 13.9 \\
\hline $3.647 \mathrm{E}+01$ & 4.9 \\
\hline$-1.562 E+01$ & 72.8 \\
\hline $4.580 E-01$ & 14.7 \\
\hline $4.158 E-01$ & 27.9 \\
\hline 2. $9 B 8 E+C O$ & 8.1 \\
\hline $3.640 E-01$ & 8.9 \\
\hline 3. $171 E+00$ & 5.5 \\
\hline B. $775 E-01$ & 17.6 \\
\hline$-7.955 E-01$ & 100.8 \\
\hline 1. $293 E+O 1$ & 3.5 \\
\hline
\end{tabular}

\begin{tabular}{|c|c|}
\hline $2.228 E+03$ & \\
\hline $8.812 E+03$ & \\
\hline $8.600 E+04$ & 3.7 \\
\hline 1. $377 \mathrm{E}+\mathrm{O} 2$ & 17.5 \\
\hline 1. $170 E+O 4$ & 15.1 \\
\hline $6.679 E+03$ & \\
\hline 3. $920 E+03$ & 7.3 \\
\hline $8.685 E+01$ & 6.4 \\
\hline $3.490 E+02$ & 3.5 \\
\hline $2.251 E+O 2$ & 100.8 \\
\hline $4.0 \div \sigma E+02$ & 28.9 \\
\hline $1.831 E-01$ & 23.4 \\
\hline 1. $749 \mathrm{E}+01$ & 93.3 \\
\hline 3. $37 O E+O 2$ & 14. 1 \\
\hline $3.852 E+00$ & 9.1 \\
\hline 3. $125 E+O O$ & 2.8 \\
\hline 1. O86E+O1 & 23.5 \\
\hline $5.258 E+00$ & 10.6 \\
\hline 1. $350 E+00$ & 47.6 \\
\hline $7.41 O E+D O$ & 90.9 \\
\hline $1.034 E+00$ & 14.2 \\
\hline $3.545 E+01$ & 3.8 \\
\hline $5.027 E+00$ & 4. 8 \\
\hline $2.77 .4 E+O O$ & 6.4 \\
\hline $4.750 E-01$ & 7.0 \\
\hline 1. $684 E+00$ & 54.7 \\
\hline 1. $144 E-02$ & 100.2 \\
\hline $1.201 E+01$ & 3.8 \\
\hline $4.962 \mathrm{E}+01$ & 5.6 \\
\hline $3.236 E+04$ & 3.8 \\
\hline $1.126 E+01$ & 4.6 \\
\hline 1. $408 E+02$ & 11.4 \\
\hline $1.812 E+00$ & 101.4 \\
\hline $1.353 E+02$ & 7.4 \\
\hline $.55 O E+O 2$ & 71.5 \\
\hline $2.944 E+O O$ & 65.5 \\
\hline $5.536 E-01$ & 78.9 \\
\hline 1. $220 E+01$ & 5.3 \\
\hline $3.911 E+O 2$ & 15.1 \\
\hline $7.052 E+01$ & 3.9 \\
\hline $.300 E+01$ & 78.5 \\
\hline $3.298 E-01$ & 9.6 \\
\hline $.293 E-01$ & 17.6 \\
\hline 3. $182 E+O O$ & 7.2 \\
\hline $.182 E-01$ & 6.7 \\
\hline $1.443 E+00$ & 4.7 \\
\hline $1.206 E+00$ & 13.6 \\
\hline $.011 E+00$ & 100.8 \\
\hline $.478 \mathrm{E}+01$ & 3.4 \\
\hline
\end{tabular}

5. $163 E+03$

1. $912 E+O 3$ 3. $887 \mathrm{E}+04$

7. $392 \mathrm{E}+\mathrm{O}$

. $449 \mathrm{E}+03$

1. $269 \mathrm{E}+04$

2. $089 E+03$

2. $425 \mathrm{E}+0$

4. $327 \mathrm{E}+02$

$-2.4 / 4 \mathrm{E}+\mathrm{O} 2$

$2.933 E+O 2$

$1578 E-01$

1. $922 \mathrm{E}+\mathrm{O}$

5. $707 E+02$

4. $012 E+00$

5. $808 \mathrm{E}+00$

$-1.475 E+O$

4. $808 \mathrm{E}+00$

4. $290 E+00$

1. $O B 3 E+O 1$

. $093 \mathrm{E}+00$

2. $329 E+O$

3. $34 O E+O O$

3. $573 E+00$

6. 709E-OI

2. $240 E+00$

1. 397E-O2

5. $199 E+00$

1. $165 E+01$

1. $61 \mathrm{ZE}+03$

6. 999E-01

$-3.150 E+O O$

3. $342 \mathrm{E}+00$

4. $392 \mathrm{E}+\mathrm{O}$

$-1.867 E+02$

- ? 16BE+OO

1. $031 E+00$

$1.417 E+00$

$6.058 E+02$

5. $004 \mathrm{E}+\mathrm{O}$

$-1.804 E+0$

4. $971 E-01$

. $540 E-01$

4. $545 E+00$

5. $469 \mathrm{E}-\mathrm{O}$

$5.503 E+00$

2. $295 \mathrm{E}+00$

2. $109 E+01$

93.6

25.8

20.4

12.5

5.4

oo. 8

100.3

55.9

93.2

11.1

2. 8

79.0

13.1

13. 7

14.4

4. 1

8. 4

100.2

3. 9

13.2
7.3

16.5

100. 4

44.8

15.0
71.6

64.9

32.3

i4. 5

11.3

14.7

6.2

4.5

11.1 


$\begin{array}{lc}\text { FILE ID } & 6100770 \\ \text { SAMPLE } & 033 \\ \text { DUPLICATE } & 015 \\ \text { CLUSTER } & 09 \\ \text { TYPE } & \text { L }\end{array}$

ELEMENT

$\mathrm{Na}-24$

$\mathrm{Mg}^{-} 27$

Al- 28

Cl- 38

$\mathrm{K}-42$
$\mathrm{Ca}-49$

$\mathrm{Ti}-51$

$V-52$
$M n-56$

Cu- 66

$5 r-87$

$\mathrm{In}-116$
$\mathrm{I}-128$

Ba- 139

Dy -165

$U-235$

Ga- 72

$\mathrm{Br}-82$

Mo- 99

Sb- 122

La-140

$\mathrm{Sm}-153$
$\mathrm{Yb}-175$

Lu- 177

$w-187$

$5 c-46$

$\mathrm{Cr}-51$

$\mathrm{Co}-60$

$\mathrm{Co}-60$
$\mathrm{Zn}-65$

Se- 75

$\mathrm{Rb}-86$

$\mathrm{Zr}-95$

$\mathrm{Ag}-110$
$\mathrm{Sb}-124$

Cs-134

Ba -131

$\mathrm{Ce}-14$

$\mathrm{Na}-147$

Eu-152

$\mathrm{Tb}-160$

LU- 177

$\mathrm{HF}-181$

Ta-182

$\omega \quad \mathrm{Hg}-203$
6100780

034

O05

$\mathrm{L}$
6100790

035

O7
6100800

036

025

L

\begin{tabular}{|c|c|}
\hline 4. $639 E+03$ & 3.5 \\
\hline $6.513 E+03$ & 13.6 \\
\hline $7.841 E+04$ & 3.8 \\
\hline$-7.323 E+01$ & 73.3 \\
\hline $1.339 E+04$ & 13.2 \\
\hline $8.435 E+03$ & 18.5 \\
\hline $3.641 E+03$ & 7.5 \\
\hline 7. $17 \mathrm{BE}+01$ & 7.8 \\
\hline $3.014 E+02$ & 3.6 \\
\hline$-2.536 E+O 2$ & 100.8 \\
\hline$-2.706 E+02$ & 100.3 \\
\hline$-1.614 E-01$ & 55.4 \\
\hline$-1.762 E+01$ & 93.2 \\
\hline $2.805 E+02$ & 14.2 \\
\hline $4.013 E+00$ & B. 6 \\
\hline 4. $100 E+00$ & 2.8 \\
\hline$-1.281 E+01$ & 78.9 \\
\hline $2.982 E+00$ & 14.2 \\
\hline$-1.432 E+O O$ & 47.6 \\
\hline$-7.711 E+O O$ & 90.9 \\
\hline $6.810 E-01$ & 21.6 \\
\hline $3.358 E+01$ & 3.9 \\
\hline $5.054 E+00$ & 4.6 \\
\hline $2.461 E+00$ & 7.9 \\
\hline $3.791 E-01$ & 12.1 \\
\hline$-1.803 E+00$ & 54.4 \\
\hline$-1.207 E-02$ & 100.2 \\
\hline $9.809 E+00$ & 3.9 \\
\hline $3.695 E+01$ & 6.8 \\
\hline $2.584 E+O 4$ & 3.9 \\
\hline $9.47 t E+O O$ & 4.9 \\
\hline $1.086 E+02$ & 13.1 \\
\hline$-2.151 E+\infty 0$ & 64.7 \\
\hline $9.595 E+01$ & 9.6 \\
\hline $3.472 E+02$ & 24.9 \\
\hline$-2.542 E+00$ & 65.1 \\
\hline$-6.599 E-01$ & 82.3 \\
\hline $1.072 E+01$ & 5.5 \\
\hline $2.687 E+O 2$ & 21.4 \\
\hline $6.637 E+01$ & 4.5 \\
\hline $3.704 E+01$ & 28.8 \\
\hline $9.164 E-01$ & 10.3 \\
\hline $6.422 E-01$ & 29.2 \\
\hline $2.478 E+O O$ & 9.0 \\
\hline $4.388 E-01$ & 7.8 \\
\hline $5.677 E+00$ & 4.5 \\
\hline $1.262 E+00$ & 15.0 \\
\hline$-9.282 \mathrm{E}-01$ & 100.8 \\
\hline $1.556 E+01$ & \\
\hline
\end{tabular}

\begin{tabular}{|c|c|}
\hline $1.416 E+O 3$ & 4.5 \\
\hline $3.294 \mathrm{E}+03$ & 17.5 \\
\hline $2.479 E+04$ & 5.2 \\
\hline $2.292 E+O 2$ & 12.9 \\
\hline$-3.618 E+03$ & 97.3 \\
\hline $8.411 E+03$ & 17.6 \\
\hline $1.450 E+03$ & 16.2 \\
\hline 4. $142 \mathrm{E}+\mathrm{O} 1$ & 10.3 \\
\hline $4.371 E+O 2$ & 3.5 \\
\hline$-2.149 E+O 2$ & 100.8 \\
\hline$-3.041 E+02$ & 100.3 \\
\hline $2.523 \mathrm{E}-01$ & 20.6 \\
\hline$-1.965 E+01$ & 93.3 \\
\hline$-1.346 E+02$ & $100 . t$ \\
\hline $2.622 \mathrm{E}+00$ & 13.7 \\
\hline $1.919 E+O O$ & 2.8 \\
\hline$-9.685 E+00$ & 79.2 \\
\hline 6. $188 E+\infty 0$ & 9.4 \\
\hline $2.561 E+00$ & 16.1 \\
\hline$-6.196 E+00$ & 90.6 \\
\hline $5.242 \mathrm{E}-0$ & 16.5 \\
\hline $2.016 E+01$ & 4.2 \\
\hline $2.53: E+O O$ & 4.9 \\
\hline $2.722 E+O O$ & 6.0 \\
\hline $3.794 E-01$ & 8. 1 \\
\hline $2.869 E+00$ & 15.6 \\
\hline$-9.133 E-03$ & 100.2 \\
\hline $4.293 E+00$ & 3.9 \\
\hline $1.789 E+01$ & 8.0 \\
\hline 1. $966 E+O 4$ & 3.9 \\
\hline $4.54 O E+O O$ & 5.8 \\
\hline $7.838 E+01$ & 13.2 \\
\hline $2.71 O E+O O$ & 29.9 \\
\hline $2.954 E+O 1$ & 21.1 \\
\hline$-1.338 E+02$ & 95.0 \\
\hline$-1.983 E+00$ & 65.6 \\
\hline$-5.511 E-01$ & 65.4 \\
\hline $2.254 E+00$ & 11.2 \\
\hline $1.331 E+O 2$ & $2 \varepsilon .0$ \\
\hline $3.271 \mathrm{E}+\mathrm{O} 1$ & 4.2 \\
\hline$-9.688 E+00$ & 77.3 \\
\hline $4.027 E-01$ & 14.2 \\
\hline $4.554 E-01$ & 16.3 \\
\hline $2.853 E+O O$ & 6.5 \\
\hline $4.263 E-01$ & 6.4 \\
\hline $2.819 E+O O$ & 5.7 \\
\hline $8.222 E-01$ & 17.2 \\
\hline$-6.948 E-01$ & 100.8 \\
\hline & \\
\hline
\end{tabular}

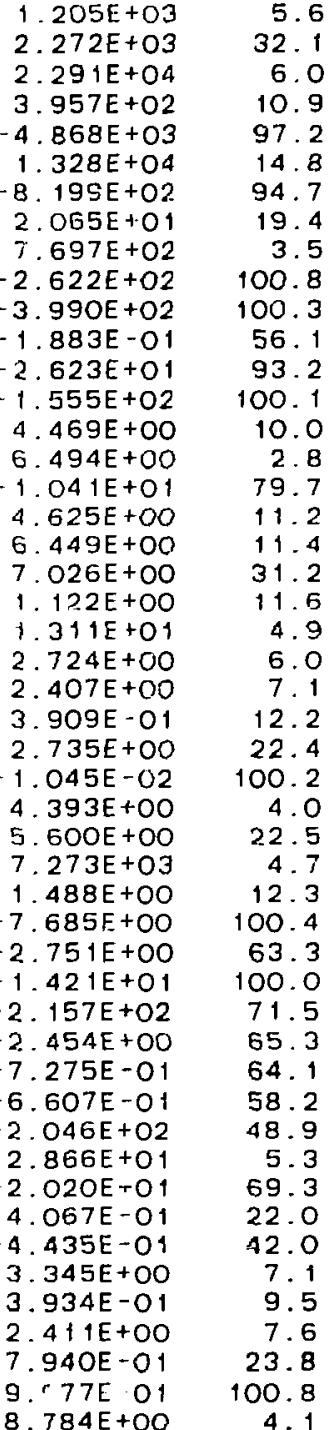

1. $158 E+O 3$

4. $400 E+O 4$

B. $315 \mathrm{E}+01$

2. $552 \mathrm{E}+\mathrm{O} 3$

1. $299 E+04$

1. $567 E+03$

2. $952 \mathrm{E}+\mathrm{O} 1$

$.502 E+O 2$

1. $944 E+02$

2. $723 \mathrm{E}+\mathrm{O} 2$

$-1.347 E-01$

$-1.772 \mathrm{E}+\mathrm{O}$

-1. 190E +02

2. $912 \mathrm{E}+\mathrm{OO}$

$3.962 \mathrm{E}+00$

$6.498 \mathrm{E}+00$

4. $718 E+0 O$

3. $848 \mathrm{E}+\mathrm{OO}$

$4.669 \mathrm{E}+00$

1. $003 E+00$

97 $1 E+O 1$

3. $24 O E+O O$

2. $15 \mathrm{E}+00$

3. $115 \mathrm{E}-\mathrm{O}$

2. $593 \mathrm{E}+00$

$-6.670 \mathrm{E}-03$

5. $062 E+00$

. $287 E+0$

8. $301 E+03$

2. $233 \mathrm{E}+\mathrm{OO}$

$5.613 E+O 1$

4. $293 E+O 0$

1. $979 E+O$

$-1.331 E+O 2$

$-1.565 E+00$

1. $049 E+O O$

2. $428 E+O O$

1. $197 \mathrm{E}+02$

4. 155E+O

$-8.500 E+O O$

4. 207E-0 1

4. $842 \mathrm{E}-\mathrm{O} 1$

2. $386 E+O O$

3. $810 E-O 1$

2. $955 \mathrm{E}+\mathrm{OO}$

1. $036 \mathrm{E}+\mathrm{OO}$

$-5.899 E-01$

4.1

22.8

35.4

33.5

12.0

11.3
3.5

100.8

100.3

93.3

100.1

13.0

2.8

9.9

9.9

90.

90.3

4.0

4. 8

10.4

17.3

00.2

3.9

9. 1

7.0
14.4

21.6

18.7

71.6

65.1

24.7

65.4

4.3

76.4

$\begin{array}{r}6.8 \\ \hline\end{array}$

6. 9

12.0

100.8
3.3 
6. $084 E+03$

5. $598 \mathrm{E}+\mathrm{O} 3$

$6.249 E+O 4$

9. $052 \mathrm{E}+\mathrm{O}$

1. $411 \mathrm{E}+\mathrm{OA}$
4. $383 \mathrm{E}+03$

3. $309 E+03$

$5.528 \mathrm{E}+01$

2. $074 \mathrm{E}+02$

2. $042 E+02$

1. $985 \mathrm{E}+\mathrm{O} 2$

-1 . 199E-01

1. $288 E+O 1$

9. $476 E+O O$

9. $376 E+O O$

1. $145 \mathrm{E}+01$

9. $554 \mathrm{E}+00$

1. $47 \mathrm{OE}+\mathrm{OO}$

$7.470 E+0 O$

9. $522 \mathrm{E}-\mathrm{O} 1$

5. $882 E+O 1$

9. $44 O E+O O$

5. $810 E+00$

8. $631 \mathrm{E}-\mathrm{O} 1$

- $.85 \& E+O 0$

$-1.062 E-02$

7. $17 \mathrm{BE}+\mathrm{OO}$

3. $610 E+01$

2. $239 E+04$

8. $109 E+00$

7. $485 \mathrm{E}+01$

2. $041 \mathrm{E}+\mathrm{OO}$

7. $603 \mathrm{E}+\mathrm{O} 1$

3. $469 \mathrm{E}+\mathrm{O} 2$

1. $390 \mathrm{E}+\mathrm{OO}$

9. $386 \mathrm{E}-01$

6 $707 \mathrm{E}+0$

$4.945 E+O 2$

1. $189 \mathrm{E} \div \mathrm{O} 2$

3. $481 \mathrm{E}+\mathrm{O}$

1. $775 \mathrm{E}+00$

1. $184 \mathrm{~F}+00$

$6.415 E+00$

8. $867 \mathrm{E}-01$

1. $142 \mathrm{E}+01$

1. $257 E+00$

6. $385 E-O$
3. 1

12.8

3.6
23.5

11.5

16.6
6.6

6. 6

3.6

100. 8

55.7

93.1

6.8

2. 8

27.0

25.

90.6

3.2

3.6
4.5

4.8

5.3

54.7

3. 8

5.9

4.6

13.4

7.9

17.1

99.2

23.7

11.5

3.8
23.0

6.3

10.4

4.7

3. 5

11.8
100.8

3.3
4. $887 \mathrm{E}+03$

4. $322 E+03$

$6.33 \mathrm{BE}+04$

1. $134 \mathrm{E}+04$

1. $134 \mathrm{E}+04$

6. BO2E+O3

2. $915 E+O 3$

$.069 \mathrm{E}+01$

2. $394 E+02$

2. $356 E+02$

2. $450 E+02$

1. 526E-O1

1. $594 E+01$

$.913 E+O 2$

4. $507 E+00$

1. $438 E+01$

3. $326 E+O O$

. $578 E+00$

- B. 153E+OO

. $429 E-O 1$

4. $408 E+01$

3. $256 E+O O$

OSOE-O1

- . $934 E+00$

-1. $260 E-O 2$

6. $784 E+O O$

3. $746 E+01$

2. $310 E+04$

7. $148 E+O O$

1. $35 B E+O 2$

4. $168 E+O D$

$358 E+01$

$.675 E+02$

$-2.262 \mathrm{E}+00$

$-5.927 \mathrm{E}-\mathrm{O}$

$6.055 \mathrm{E}+0 \mathrm{O}$

2. $897 E+02$

9. $390 E+01$

1. $153 \mathrm{E}+\mathrm{O} 1$

$111 E+O O$

7. $145 E-O 1$

3. $808 \mathrm{E}+00$

5. 215E-01

8. $089 E+00$

1. $521 E+O O$

8. $342 \mathrm{E}-01$
3. 3

18.2

25.5

15.3

18.8

9.1

3.6

100.8

100.3

22.7

13. 6

+0. 0

2.8
79.0

12.3
47.5

91.0

4.8

6.2

8.5

49.2

100. 2 
om

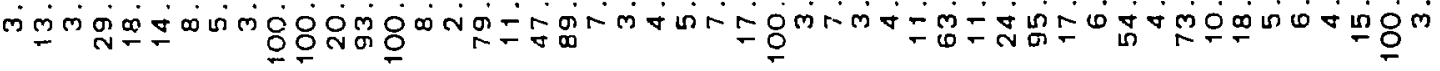

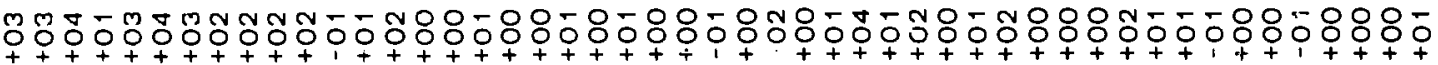

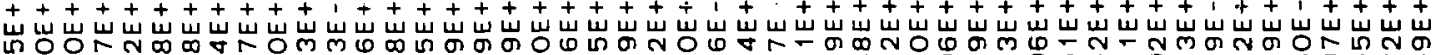

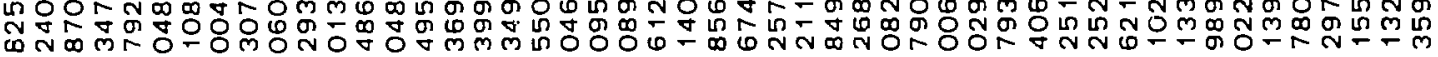
n

2

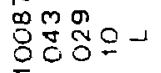
6

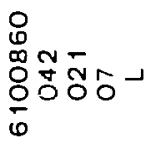

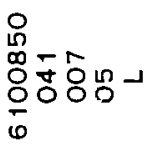

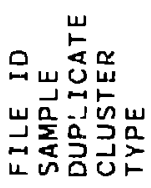

movdm

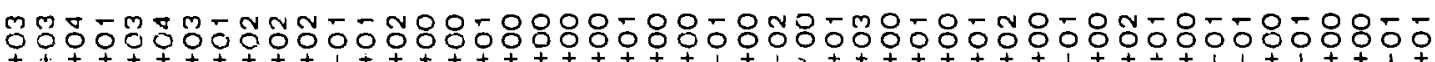

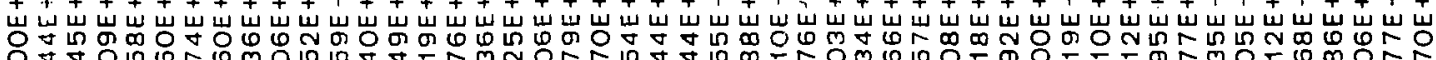

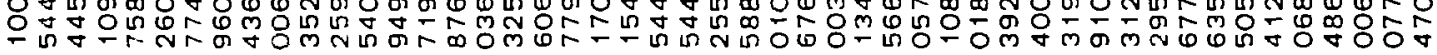

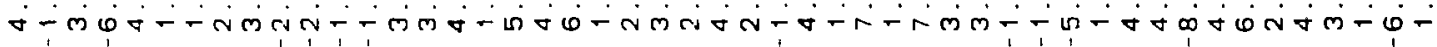

mot 0 m

món

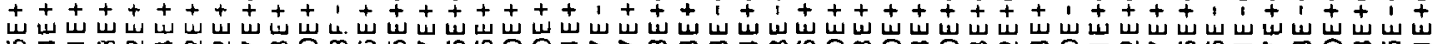
LV=

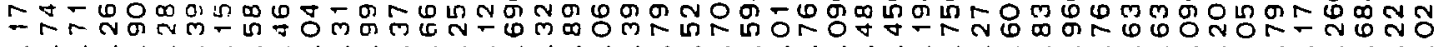
mवन

mmathn mะ

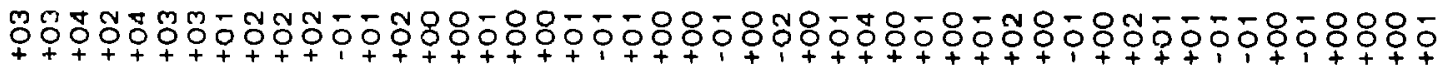
س山 س山س

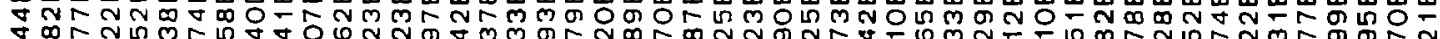

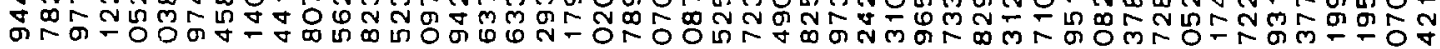

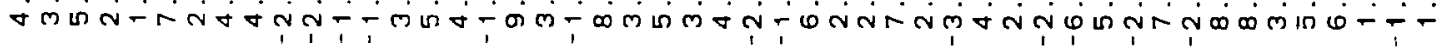

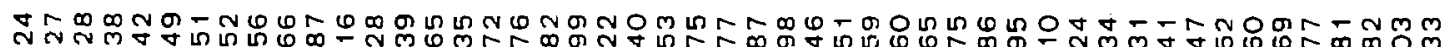

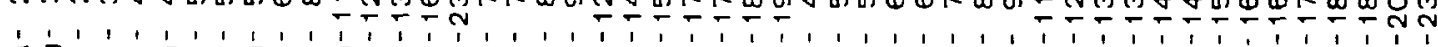

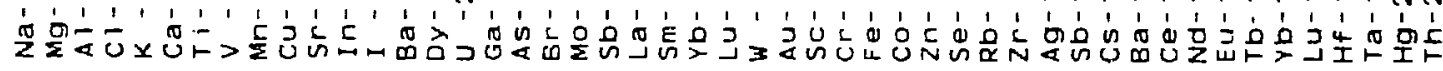


우

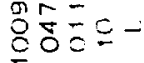
6
का

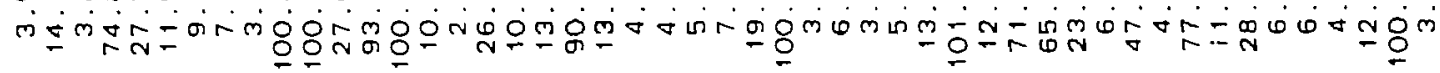

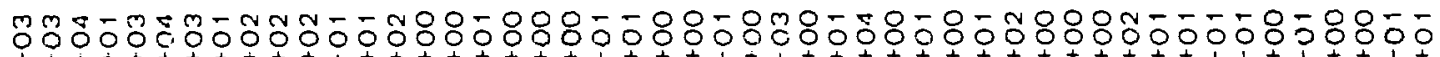

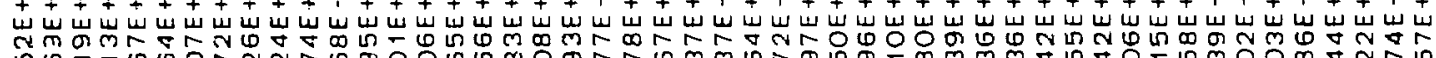

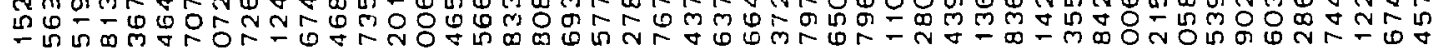

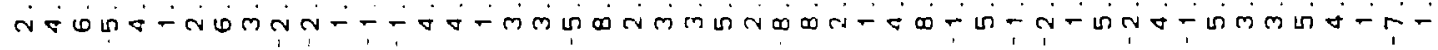

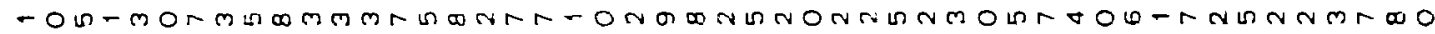
ம

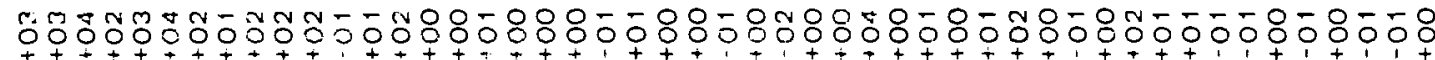

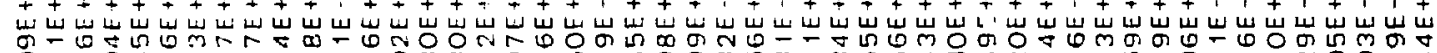

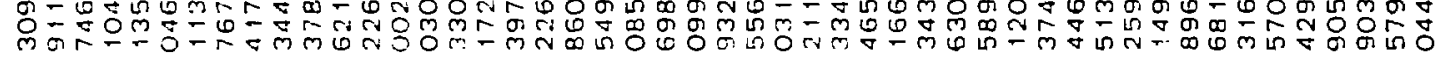

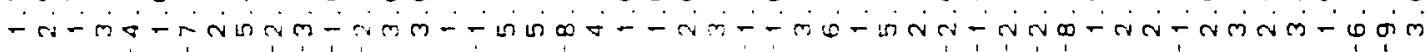

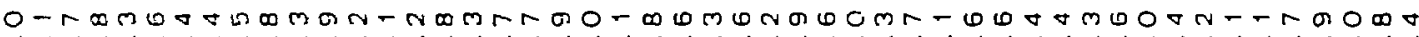
va

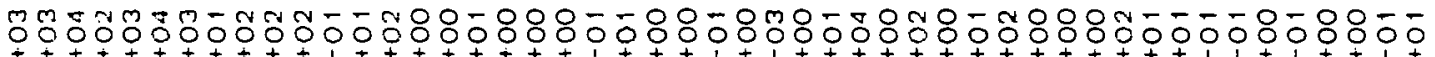

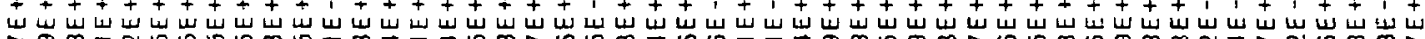

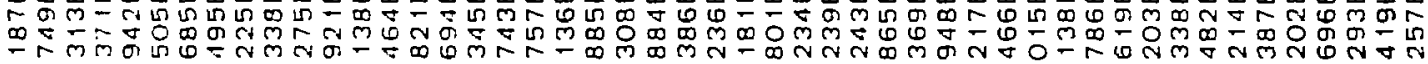
d-m

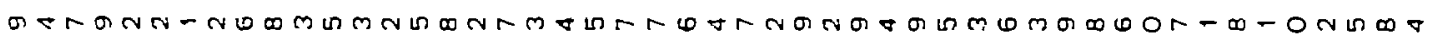

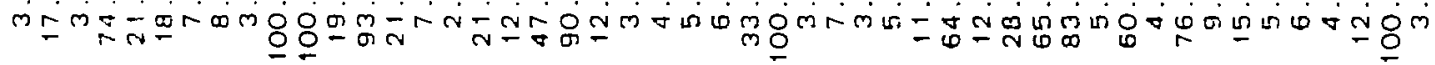

웅

פำ

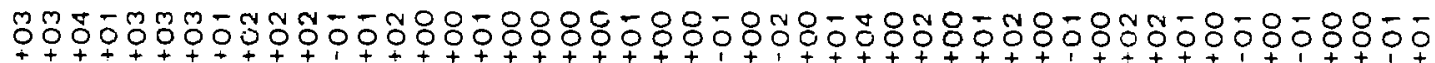

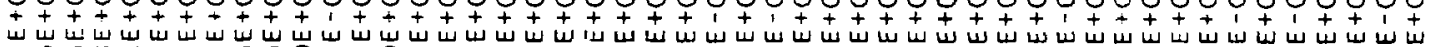

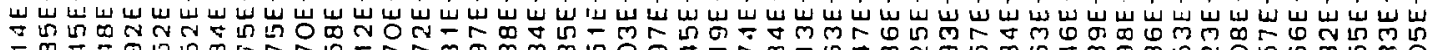

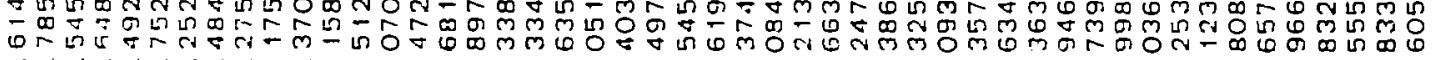

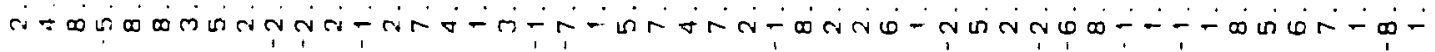

\section{는 \\ un

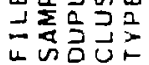

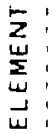

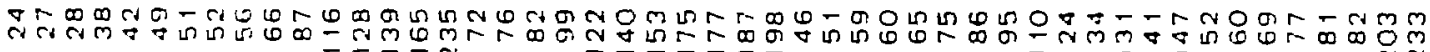




$\begin{array}{lc}\text { FILE TD } & 6100930 \\ \text { SAMPLE } & 049 \\ \text { DUPLICATE } & 028 \\ \text { CLUSTER } & 06 \\ \text { TYPE } & L\end{array}$

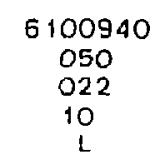

10
6100950

051

09

O9
6100960

052

07

ELEMENT

$\mathrm{Na}-24$

$\mathrm{Mg}-27$

A) - 28

C I - 38

$\mathrm{K}-42$
$\mathrm{Ca}-49$

$\mathrm{Ti}-51$

$v-52$
$\mathrm{Mn}-56$

$\mathrm{Cu}-66$

Sr- 87

In- 116

Ba-139

Dy -165

$\mathrm{Ga}-72$

$A s-76$

$\mathrm{Br}-82$
$\mathrm{Mo}-99$

Sb- 122

La- 140

Sm- 153

$Y b-175$
$L U-177$

W- 187

Au- 198

SC- 46

$\mathrm{Fe}-59$

$\mathrm{Fe}-59$

7n- 65

$\mathrm{Zn}-65$
$5 e-75$

$\mathrm{Rb}-86$

$\mathrm{Zr}-95$

$\mathrm{Ag}-110$

5b-124

Cs-134

$\mathrm{Ba}-131$

$\mathrm{Ce}-14$

Nd-147

Eu-152

TO- 160

LU-177

Hf - 181

Ta-182

$\mathrm{Ta}-182$
$\mathrm{Hg}-203$

w $\mathrm{Hg}-203$

\begin{tabular}{|c|c|}
\hline $3.729 E+03$ & 3.6 \\
\hline$-2.012 E+03$ & 93.7 \\
\hline 3. $246 E+O 4$ & 4.9 \\
\hline$-7.334 E+01$ & \\
\hline $6.622 \mathrm{E}+03$ & 24.3 \\
\hline 1. $481 E+04$ & \\
\hline 1. $.532 \mathrm{E}+03$ & 16. \\
\hline $1.535 E+01$ & 19.3 \\
\hline 4. $488 E+O 2$ & \\
\hline$-2.512 \mathrm{E}+\mathrm{O} 2$ & 100.8 \\
\hline$-3.206 E+02$ & 100.3 \\
\hline$-1.642 E-01$ & 56.1 \\
\hline$-2.12 B E+01$ & 93.2 \\
\hline$-1.44 \mathrm{OE}+\mathrm{O} 2$ & 100.1 \\
\hline$-1.284 E+O O$ & 67.3 \\
\hline 5. $149 E+O O$ & 2.8 \\
\hline$-1.584 E+01$ & 79.0 \\
\hline $6.606 E+00$ & 10.8 \\
\hline $4.758 E+00$ & 13.1 \\
\hline$-1.064 E+01$ & 89.6 \\
\hline 1. $115 \mathrm{E}+\infty$ & 11.8 \\
\hline $1.787 \mathrm{E}+01$ & 4.4 \\
\hline $2.514 \mathrm{E}+00$ & 5. \\
\hline $2.239 E+O O$ & 9.1 \\
\hline $3.287 E-01$ & 13.3 \\
\hline $3.089 E+00$ & 22.8 \\
\hline$-1.326 E-02$ & 100.2 \\
\hline $4.069 E+00$ & 4.0 \\
\hline $9.012 \mathrm{E}+00$ & 16.3 \\
\hline $5.783 E+03$ & 4.9 \\
\hline $7.581 E-01$ & 17.9 \\
\hline $2.570 E+01$ & 36.1 \\
\hline$-3.082 E+00$ & 58.6 \\
\hline 3. $289 E+01$ & 16. \\
\hline$-1.998 E+02$ & 71.6 \\
\hline$-2.218 E+00$ & 65.3 \\
\hline$-8.184 E-01$ & 81.8 \\
\hline $1.414 E+O O$ & 19.2 \\
\hline $1.611 E+O 2$ & 20.8 \\
\hline $3.693 \mathrm{E}+01$ & 4.5 \\
\hline $1.909 E+01$ & 72. \\
\hline $2.025 E-01$ & 32.5 \\
\hline $5.6 \mathrm{BOE}-01$ & 29.5 \\
\hline $2.132 \mathrm{E}+00$ & 13.3 \\
\hline $3.500 E-01$ & 9. \\
\hline $3.357 E+00$ & 5.5 \\
\hline 1. $147 \mathrm{E}+00$ & 17.5 \\
\hline$-9.358 E-01$ & 100.8 \\
\hline $1.459 E+01$ & \\
\hline
\end{tabular}

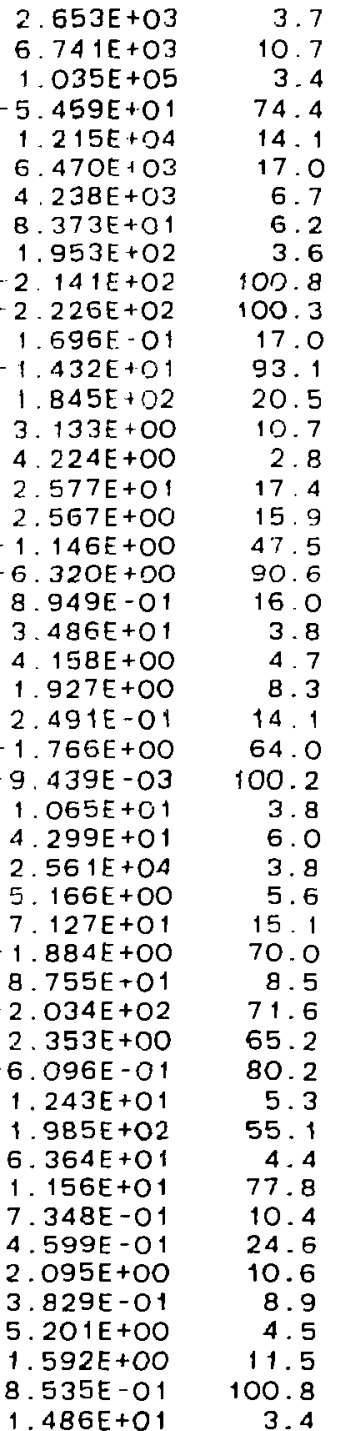

1. $181 E+03$

$-1.892 E+03$

3. $286 E+04$

2. $480 E$ rO2

4. $567 E+03$

$3 B 2 \mathrm{E}+\mathrm{O} 4$

7. $326 \mathrm{E}+\mathrm{O} 2$

2. $232 E+O$

6. $533 \mathrm{E}+\mathrm{O} 2$

2. $429 \mathrm{E}+02$

$3.557 \mathrm{E}+02$

i. $719 E-O$

2. $325 E+01$

1. $401 E+02$

$3.671 E+00$

3. $940 E+00$

1. $O 4 O E+01$

4. $210 E+O 0$

5. $970 \mathrm{E}+\mathrm{OO}$

6. $265 E+00$

1. $110 E+O O$

1. $672 \mathrm{E}+\mathrm{O} 1$

3. $129 E+O O$

3. $143 \mathrm{E}+00$

4. $847 \mathrm{E}-01$

3. $B 03 E+00$

B. $927 \mathrm{E}-03$

4. $442 \mathrm{E}+00$

1. $075 \mathrm{E}+\mathrm{O}$

1. $157 \mathrm{E}+\mathrm{O}$

1. $963 \mathrm{E}+00$

7. $131 \mathrm{E}+\mathrm{O} 1$

3. 198E +OO

1. $204 \mathrm{E}+0$

1. $734 \mathrm{E}+\mathrm{O} 2$

$1977 E+00$

$6.020 E-01$

$1.555 E+O O$

3. $337 E+O$

1. $040 E+01$

3. $389 \mathrm{E}-01$

4. 54OE-O 1

3. $288 \mathrm{E}+0 \mathrm{O}$

4. $792 \mathrm{E}-\mathrm{O}$

2. $697 \mathrm{E}+00$

8. $450 E-01$

$-7.389 E-01$

1. $194 \mathrm{E}+01$
5. 1

5.1

13.3

97.1

94.7

17.4

100.8

100.3

93.3

100.1

9.6

24.8

1.8

11.4

9.9

4. 4

5.4
5.9

7.6

16.0
100.2

3.9

11.0

B. 6
13.8

24.6

100.0

100.0

65.3

80.3

13.7

54.5

76.7

16.5
21.9

6.0

6.3
5.9

18.6

100.8
3.5 


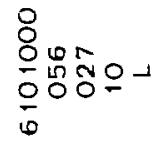

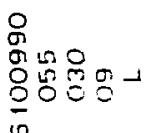

$\omega$

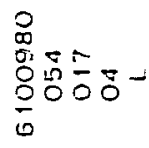

0
$80980-$
8080

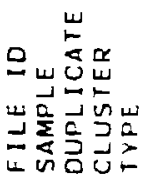

* wm

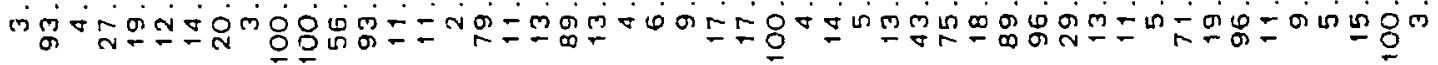

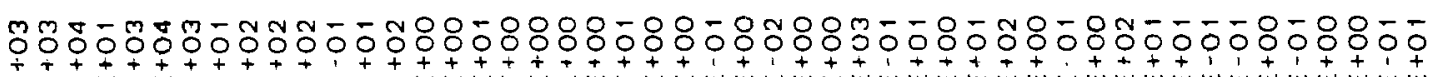

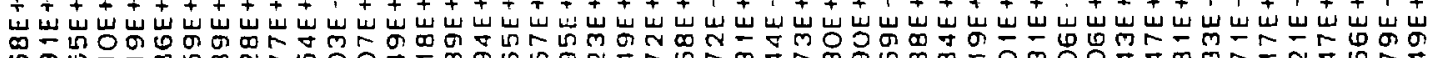

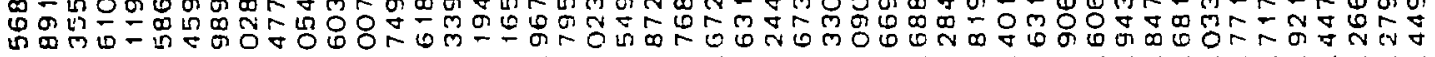

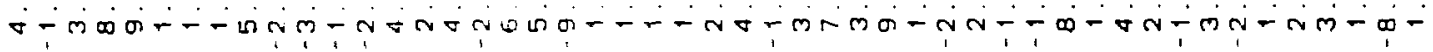

- mக mळ-

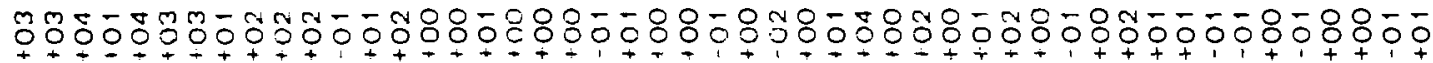

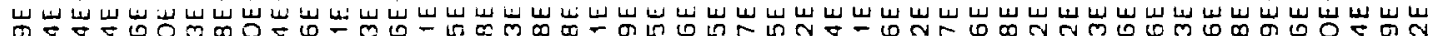

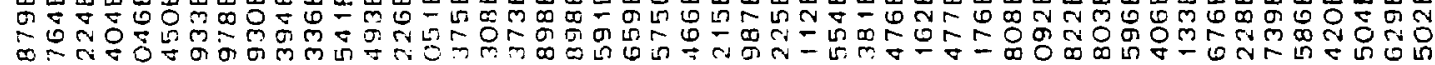

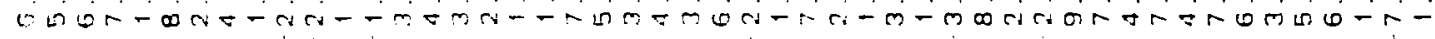

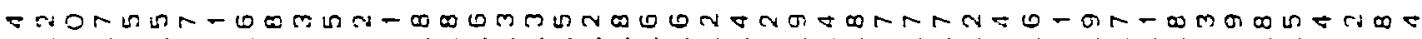
mI

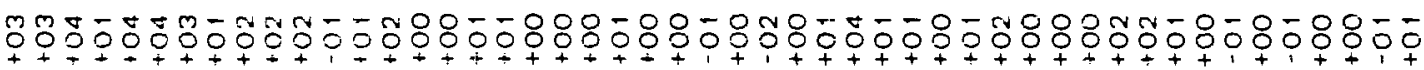

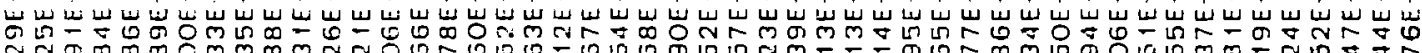

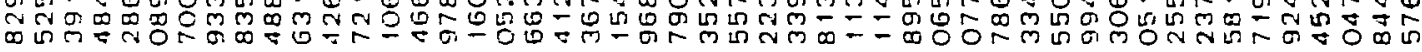

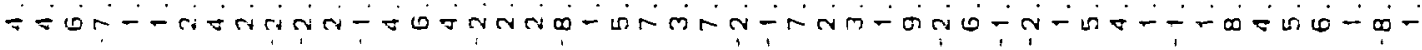

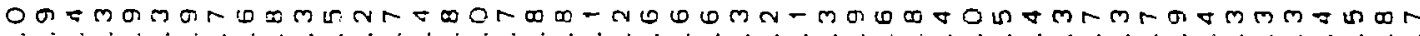
- I0-

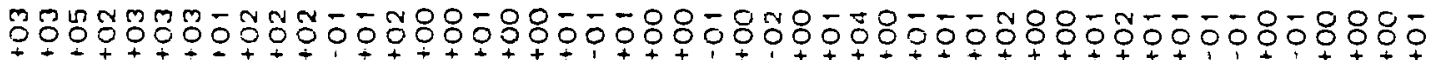

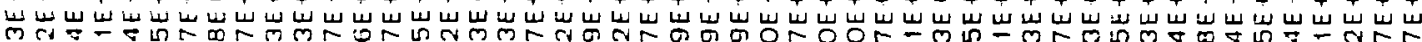

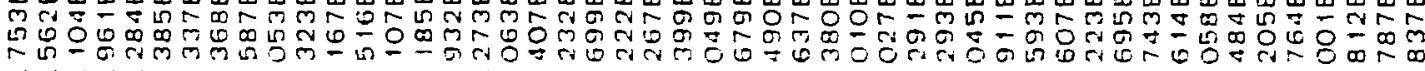

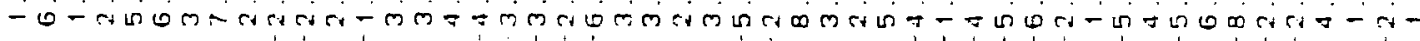

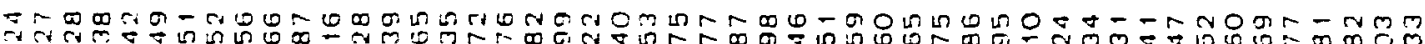
- - -

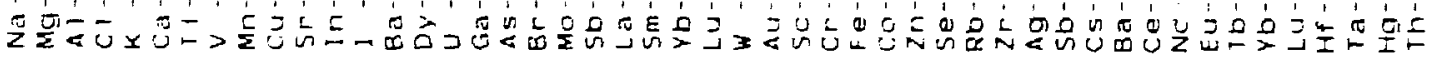


FILE ID

DUPLICATE
CLUSTER

CLUSTER
TYPE

6101010
057
023
10
$L$

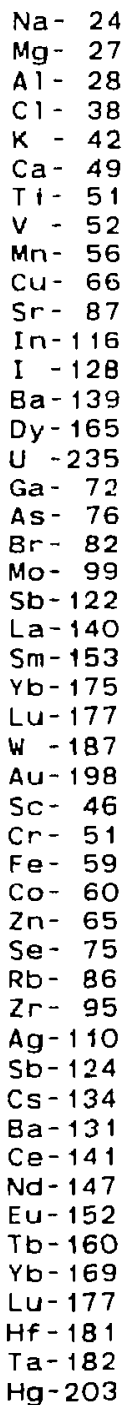

$\mathrm{Na}-24$
$\mathrm{Mg}^{-} 27$

A $1-28$

$-42$

a- 49

$-52$

Cu- 66

n- 116

Ba-139

Dy -165

Ga- 72

As- 76

Mo- 99

$5 b-122$

Sm-153

$-187$

Sc- 46

$\mathrm{Cr}-51$
$\mathrm{Fe}-59$

Co- 60

Se- 75

$\mathrm{Rb}-86$

Ag- 1 to

Sb- 124

Ba-131

$\mathrm{Ce}-141$

$\mathrm{Eu}-152$

Tb-160

Yu- 177

Ta-

$\mathrm{Hg}-203$
$\mathrm{Th}-233$
6101020

058

07
6101030

059

010

ก8
6101040

060

016
07

L

\begin{tabular}{|c|c|c|c|}
\hline 1. $150 E+03$ & 4.9 & $1.844 \mathrm{E}+03$ & 4.5 \\
\hline 2. $179 E+03$ & 30.4 & $2.887 E+03$ & 25.9 \\
\hline $2.057 \mathrm{E}+04$ & 5.6 & $2.629 E+04$ & 5.6 \\
\hline$-4.596 E+01$ & 76.2 & $5.418 E+O 2$ & 10.3 \\
\hline $3.302 \mathrm{E}+03$ & 29.4 & $-4.599 E+03$ & 97.2 \\
\hline 1. $324 E+O 4$ & 12.2 & 1. $361 \mathrm{E}+04$ & 15.5 \\
\hline$-5.152 E+O 2$ & 94.9 & $1.566 \mathrm{E}+03$ & 19.9 \\
\hline $1.679 E+01$ & 15.3 & $4.487 E+01$ & 12.0 \\
\hline $3.173 \mathrm{E}+\mathrm{O} 2$ & 3.6 & $6.613 \mathrm{E}+02$ & 3.5 \\
\hline$-1.755 E+O 2$ & 100.8 & $-2.652 \mathrm{E}+\mathrm{O} 2$ & 100.8 \\
\hline$-2.479 E+O 2$ & 100.3 & $-3.715 E+O 2$ & 100.3 \\
\hline $1.293 E-01$ & 28.0 & $-1.796 E-01$ & 56.1 \\
\hline$-1.619 E+01$ & 93.3 & $-2.473 E+01$ & 93.3 \\
\hline 3. $13 B E+O 2$ & 14.5 & $-1.480 E+O 2$ & 100.1 \\
\hline $2.037 E+O O$ & 15.2 & $3.840 E+00$ & 10.0 \\
\hline $1.784 E+O O$ & 2.8 & $2.447 E+O O$ & 2.8 \\
\hline$-1.129 E+O 1$ & 79.8 & $-1.924 E+01$ & 79.6 \\
\hline $2.436 E+O O$ & 14.8 & $3.069 E+00$ & 16.1 \\
\hline $5.564 \mathrm{E}+00$ & 10.4 & $5.231 E+O O$ & 14.6 \\
\hline$-4.631 E+O O$ & 90.1 & $-7.195 E+00$ & 90.3 \\
\hline $2.69 ! \mathrm{E}-01$ & 26.0 & $7.231 E-01$ & 14.2 \\
\hline B. $527 E+O O$ & 5.4 & 1. $709 \mathrm{E}+01$ & 4.6 \\
\hline $1.695 E+00$ & 4.9 & 2. $951 \mathrm{E}+\mathrm{OO}$ & 4.9 \\
\hline $1.284 E+0 O$ & 9.3 & $3.339 E+O O$ & 6.1 \\
\hline $1.963 E-01$ & 11.3 & $6.072 \mathrm{E}-01$ & 6.2 \\
\hline $2.00 t E+00$ & 24.6 & $4.958 E+00$ & 16.2 \\
\hline$-6.423 E-03$ & 100.2 & $-1.013 E-02$ & 100.2 \\
\hline $2.886 E+O O$ & 4.0 & $6.064 E+O O$ & 3.9 \\
\hline $7.407 E+O O$ & 11.0 & $1.657 E+01$ & 9.4 \\
\hline $4.666 E+03$ & 4.8 & $6.090 E+03$ & 4.6 \\
\hline $9.678 E-01$ & 12.0 & $2.544 E+O O$ & 7.7 \\
\hline $2.954 E+01$ & 23.0 & $5.191 E+01$ & 17.5 \\
\hline$-2.029 E+00$ & 68.6 & $3.577 E+\infty 0$ & 25.3 \\
\hline $2.677 \mathrm{E}+01$ & 17.2 & $-1.195 E+01$ & 100.0 \\
\hline$-1.159 E+02$ & 71.5 & $2.280 E+02$ & 31.6 \\
\hline$-1.305 E+00$ & 65.3 & $-2.244 E+O O$ & 64.7 \\
\hline$-4.485 E-01$ & 79.7 & $-7.029 E-01$ & 63.1 \\
\hline $1.028 E+00$ & 17.0 & $2.181 E+O O$ & 14.1 \\
\hline $3.478 \mathrm{E}+\mathrm{O} 2$ & 11.7 & $-1.841 E+02$ & 55.0 \\
\hline $1.905 \mathrm{E}+01$ & 5.5 & 3. $306 \mathrm{E}+01$ & 4.7 \\
\hline$-7.835 E+00$ & 75.7 & $-1.0 B 4 E+01$ & 77.6 \\
\hline $3.362 \mathrm{E}-01$ & 17.0 & 5. $164 \mathrm{E}-01$ & 13.9 \\
\hline $2.956 E-01$ & 24.4 & $6.634 \mathrm{E}-01$ & 18.5 \\
\hline $1.416 E+O O$ & 10.0 & $3.799 E+0 O$ & 5.6 \\
\hline $3.009 E-01$ & 8.8 & $5.869 E-01$ & 6.0 \\
\hline $2.132 \mathrm{E}+O \mathrm{O}$ & 6.2 & 2. $906 \mathrm{E}+00$ & 5.9 \\
\hline $4.774 E-01$ & 18.2 & $7.651 E-01$ & 25.2 \\
\hline$-5.262 E-01$ & 100.8 & $-7.97 B E-01$ & 100.8 \\
\hline & & $8.463 E+00$ & 3.8 \\
\hline
\end{tabular}

1. $167 E+03$ 1. $907 E+03$

2. $183 E+O A$

2. $490 E+O 2$

$-4.500 E+03$

. $619 E+D 4$

1. $38 \mathrm{BE}+03$

3. $056 E+01$

6. $669 E+02$

2. $485 \mathrm{E}+\mathrm{O} 2$

$-3.63 \mathrm{OE}+\mathrm{O} 2$

1. $750 E-01$

2. $376 \mathrm{E}+01$

1. $45 O E+02$

3. $405 E+00$

2. $402 E+00$

1. $407 E+O 1$

4. $458 E+O O$

5. $525 \mathrm{E}+0 \mathrm{O}$

$.021 E-01$

. $713 E+O O$

. $993 E+00$

. $651 E+00$

4. 353E-O 1

$3.063 E+00$

$-7.830 E-03$

$5.452 E+O O$

1. $117 \mathrm{E}+\mathrm{O} 1$

. $103 E+00$

. $287 E+01$

3. $248 E+O O$

$-9.694 E+00$

1. $634 \mathrm{E}+\mathrm{O} 2$

$-1.939 E+00$

5. $420 E-01$

8. $780 E-01$

- $1.650 E+02$

2. $012 \mathrm{E}+\mathrm{O} 1$

$-1.029 \mathrm{E}+01$

4. $045 E-01$

3. $137 \mathrm{E}-01$

4. $542 \mathrm{E}-01$

2. $219 E+O O$

4. $14 \mathrm{E}-01$

$-7.105 E-01$

6. $467 \mathrm{E}+00$
5. 2

25.4

13. 1

97.2

13.8
19.5

10. 7

3.5
00.8

100.3

30.0

93.4

100.1

10.9

2.8

80.0

14.4

90.2

17.3

5.4

6.6

7.8

18.9

3.9

11.1

4. 5

24.4

22.3

100.0

71.7
64.9

64.0

28.3

55.4

42.2

5.9

6. 9

38.7

00.8 
mषகमूं

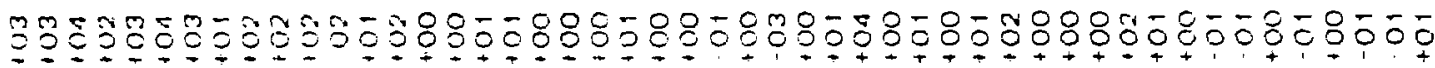

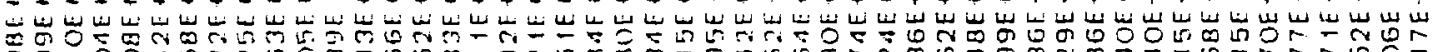

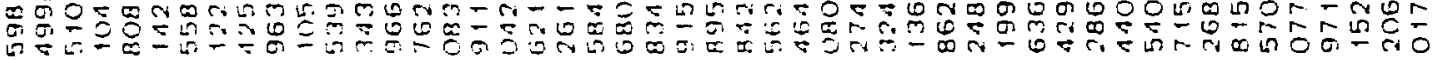

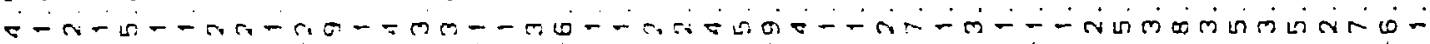

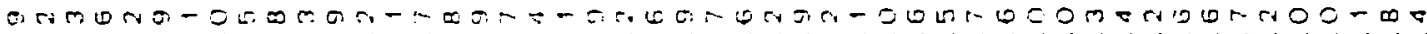
वर

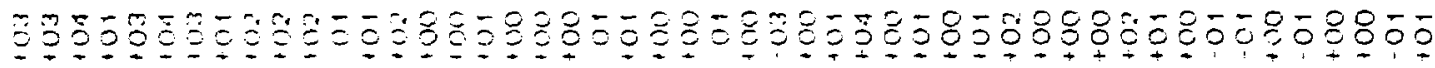

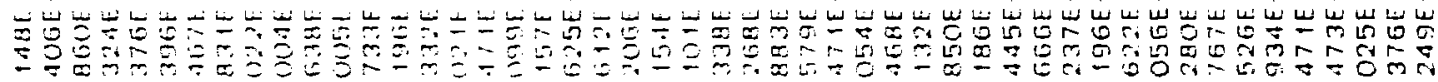

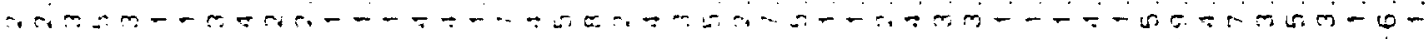

20,-

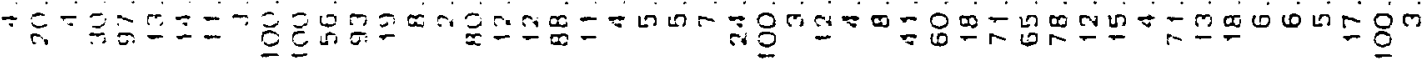

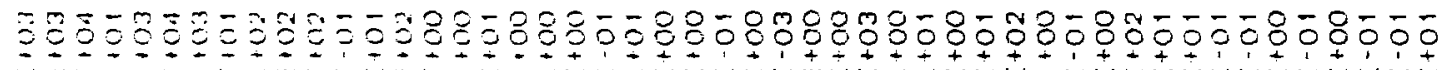

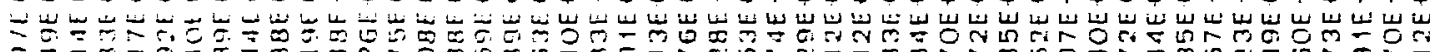

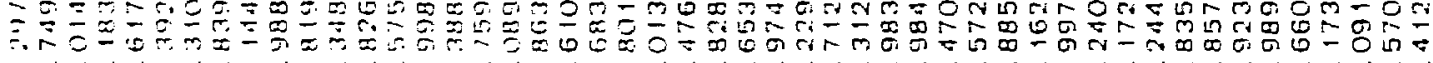

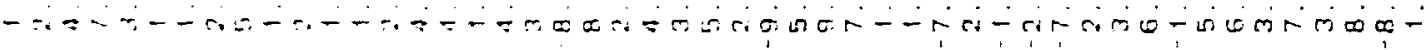

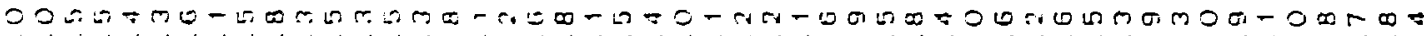
ح

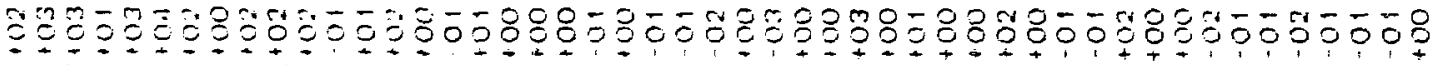

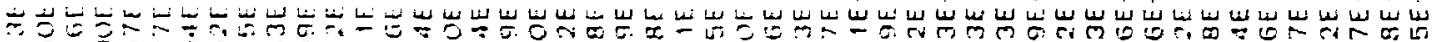

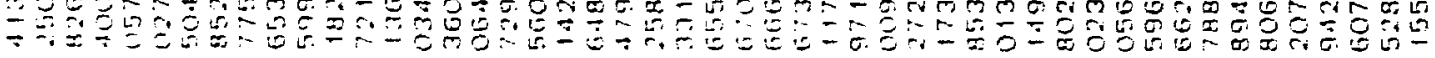

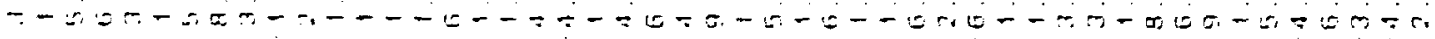

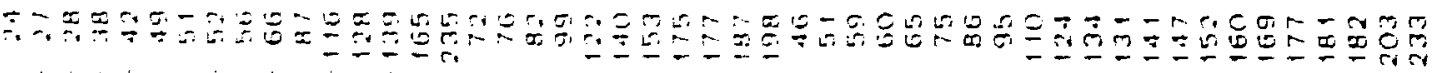

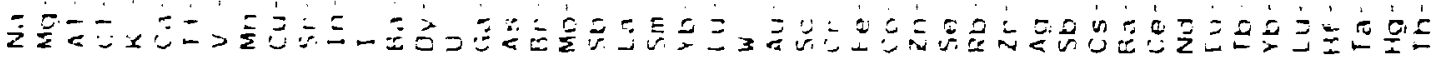




$\begin{array}{lc}\text { FILE ID } & 6101090 \\ \text { SAMPLE } & 065 \\ \text { DUPLICATE } & 130 \\ \text { CLUSTER } & 10 \\ \text { TYPE } & L\end{array}$

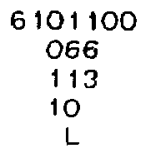

666

10
6101110

067

01
6101120

068

10

ELEMENT

$\mathrm{Na}-24$

$\mathrm{Mg}-27$

A) -28

Cl- 38

$k-42$

$\mathrm{Ca}-49$
$\mathrm{~T} i-51$

$T i-51$
$v-52$

Mn- 56

$\mathrm{Cu}-66$
$\mathrm{Sr}-87$

Sr- 87
Ir $1-116$

$I r_{1}-116$
$I-128$

Ba-139

$D y-165$
$U-235$

Ga- 72

As- 76

$\mathrm{Br}-82$

Sb- 122

La-140

$5 \mathrm{~m}-153$

Yb- 175

LU- 177

$w-187$

Au- 198

$\mathrm{Sc}-46$
$\mathrm{C} r-51$

$\mathrm{Fe}-59$

Co- 60

Zn- 65

$\mathrm{Se}-75$

Zr- 95

Ag- 110

Sb- 124

Cs-134

$\mathrm{Ba}-131$

Ce- 141

No-147

Eu- 152

Y
$Y \mathrm{D}-169$

Y

Hf -181

$\mathrm{Ta}-182$

$\mathrm{Hg}-203$
$\mathrm{Th}-233$

\begin{tabular}{|c|c|}
\hline $\begin{array}{l}1.077 \mathrm{E}+03 \\
2.254 \mathrm{E}+03\end{array}$ & $\begin{array}{r}4.8 \\
25.7\end{array}$ \\
\hline $3.817 \mathrm{E}+04$ & 4.3 \\
\hline $1.127 E+O 2$ & 22.1 \\
\hline$-3.403 E+03$ & 97.2 \\
\hline $1.473 E+O 4$ & 12.6 \\
\hline 1. $523 E+03$ & 14.6 \\
\hline $1.922 \mathrm{E}+01$ & 14.0 \\
\hline 4. $282 E+O 2$ & 3.5 \\
\hline$-1.920 E+02$ & 100.8 \\
\hline$-2.678 E+02$ & 100.3 \\
\hline 1. $191 \mathrm{E}-01$ & 29.5 \\
\hline$-1.754 E+01$ & 93.2 \\
\hline $3.246 E+02$ & 18.0 \\
\hline $3.606 E+00$ & 10.7 \\
\hline $4.783 E+O O$ & 2.8 \\
\hline$-1.717 E+01$ & 79.9 \\
\hline 8. $40 A E+O O$ & 10.0 \\
\hline $6.081 E+00$ & 11.2 \\
\hline$-8.304 E+00$ & 88.5 \\
\hline $9.414 E-01$ & 11.1 \\
\hline 2. $152 E+O 1$ & 4.3 \\
\hline 3. $286 E+O O$ & 5.3 \\
\hline $2.826 E+00$ & 6.6 \\
\hline $4.131 E-01$ & 8.5 \\
\hline$-1.876 E+00$ & 52.7 \\
\hline$-9.514 E-03$ & 100.2 \\
\hline $4.806 \mathrm{E}+00$ & 3.9 \\
\hline $6.767 E+00$ & 20.6 \\
\hline $4.759 E+03$ & 4.9 \\
\hline $1.453 E+\infty$ & 10.3 \\
\hline $2.408 E+01$ & 33.9 \\
\hline $2.256 \mathrm{E}+O O$ & 46.0 \\
\hline$-1.149 E+01$ & 100.0 \\
\hline$-1.831 E+02$ & 71.6 \\
\hline$-2.10 O E+O O$ & 65.1 \\
\hline$-6.441 E-01$ & 77.9 \\
\hline $1.367 E+00$ & 14.4 \\
\hline $3.254 E+02$ & 15.5 \\
\hline $4.575 E+01$ & 4.5 \\
\hline 4. $303 E+01$ & 28.0 \\
\hline 4.5B7E-01 & 15.9 \\
\hline $6.859 E-01$ & 16.6 \\
\hline 3. $170 E+O O$ & 7.4 \\
\hline $4.918 E-01$ & 7.6 \\
\hline $3.083 E+00$ & 5.6 \\
\hline $1.047 \mathrm{E}+00$ & 16.0 \\
\hline$-8.371 E-01$ & 100.8 \\
\hline $1.371 \mathrm{E}+01$ & \\
\hline
\end{tabular}

$\begin{array}{rr} \\ 5.193 E+O 3 & 3.3 \\ 1.755 E+O 3 & 92.9 \\ 3.47 O E+O 4 & 4.5 \\ 1.034 E+O 2 & 17.0 \\ -3.00 O E+O 3 & 97.2 \\ 1.172 E+O 4 & 13.2 \\ 1.631 E+O 3 & 11.2 \\ 2.706 E+O 1 & 10.8 \\ 2.378 E+O 2 & 3.6 \\ -2.205 E+O 2 & 100.8 \\ -2.383 E+O 2 & 100.3 \\ 2.092 E-O 1 & 22.1 \\ -1.554 E+O 1 & 93.2 \\ 5.180 E+O 2 & 9.9 \\ 3.72 O E+O O & 10.3 \\ 4.777 E+O O & 2.8 \\ -2.146 E+O 1 & 79.3 \\ 6.555 E+O O & 10.5 \\ 3.610 E+O O & 12.8 \\ 5.343 E+O O & 15.5 \\ 1.357 E+O O & 9.8 \\ 2.231 E+O 1 & 4.2 \\ 3.576 E+O O & 5.0 \\ 3.371 E+O O & 5.7 \\ 6.446 E-O 1 & 6.4 \\ 1.157 E+O 1 & 11.5 \\ -1.069 E-O 2 & 100.2 \\ 4.9 O 8 E+O O & 3.9 \\ 8.201 E+O O & 12.9 \\ 8.188 E+O 3 & 4.2 \\ 1.702 E+O O & 7.9 \\ 9.860 E+O 1 & 11.5 \\ 2.037 E+O O & 25.1 \\ 2.152 E+O 1 & 18.9 \\ -1.565 E+O 2 & 71.6 \\ -1.800 E+O O & 65.1 \\ 1.445 E+O O & 23.4 \\ 1.351 E+O O & 13.2 \\ 4.545 E+O 2 & 8.8 \\ 4.928 E+O 1 & 4.0 \\ 2.989 E+O 1 & 25.1 \\ 4.413 E-O 1 & 13.7 \\ 8.413 E-O 1 & 13.8 \\ 3.873 E+O O & 4.7 \\ 6.26 O E-O 1 & 5.4 \\ 4.252 E+O O & 4.5 \\ 1.121 E+O O & 12.8 \\ -6.675 E-O 1 & 100.8 \\ 1.331 E+O 1 & 3.3\end{array}$

3. $151 E+0$

3. $110 E+03$

3. $082 E$ rOA

9. $065 \mathrm{E}+01$

$-4.216 E+03$

1. $115 \mathrm{E}+\mathrm{O} 4$

2. $049 \mathrm{E}+01$

2. $049 E+0$

5. O5OE +O2

-2.486E+O2

-3. $313 E+O$

$-1.696 E-01$

5. $195 E+O 2$

3. $781 \mathrm{E}+\mathrm{OO}$

3. $176 \mathrm{E}+\mathrm{OO}$

2. $468 \mathrm{E}+\mathrm{O}$

6. $277 E+00$

$5.226 E+O O$

1. $013 E+01$

1. $523 E+O O$

1. $387 \mathrm{E}+01$

2. $313 E+O O$

$2.692 \mathrm{E}+0 \mathrm{O}$

3. $700 E-01$

5. $550 E+O 0$

- 1. 244E-02

4. $522 \mathrm{E}+00$

8. $449 \mathrm{E}+\mathrm{OO}$

6. $313 \mathrm{E}+\mathrm{O} 3$

i. $272 \mathrm{E}+\mathrm{OO}$

1. $416 \mathrm{E}+01$

3. $203 \mathrm{E}+00$

2. $120 E+O$

$-2.151 E+O 2$

$-2.429 E+00$

- 8. 601E-O

1. $626 \mathrm{E}+0$

1. $851 E+02$

4. $851 E+02$

$2.865 E+O$

2.067E+O

3. $707 E-01$

5. $365 E-01$

2. $894 \mathrm{E}+\mathrm{OO}$

4. $736 \mathrm{E}-\mathrm{O} 1$

3. $139 \mathrm{E}+\mathrm{OO}$

8. $774 \mathrm{E}-01$

$-9.952 E-O 1$
3. 8

33. 1

31.4

97.1

16.1
19.7

15.2

3.5

100.8

56.1

56.1

9.6

10.2

2.8

79.4

12.0

14.4

89. 3

11.1

5.1
5.7

7.5

11.3

19.3

100.2

15.5

4.9

13.2

35.3

27.3

65.0

78.2

15.2

12.9

71.5

20.0

22.8

9.1

6.5
25.7

100.8 


\section{ELEMENT}

\begin{tabular}{|c|c|c|}
\hline $\mathrm{Na}-24$ & $2.707 E+03$ & 3.6 \\
\hline$M g-27$ & $4.564 E+03$ & 16.9 \\
\hline$A 1-28$ & 4. $991 E+04$ & 4.0 \\
\hline $\mathrm{Cl}-38$ & A. $951 E+0$ & 34.7 \\
\hline$k-42$ & $-3.549 E+03$ & 97.1 \\
\hline $\mathrm{Ca}-49$ & $1.356 E+04$ & 13.0 \\
\hline$T i-51$ & 1. $308 \mathrm{E}+03$ & 14.2 \\
\hline$v-52$ & 2. $804 E+01$ & 10.6 \\
\hline$M n-56$ & 4. $1 A 4 E+O 2$ & 3.5 \\
\hline Cu- 66 & $-2.091 E+02$ & 100.8 \\
\hline$S r-87$ & $-2.693 E+02$ & 100.3 \\
\hline In-116 & $-1.396 E-01$ & 55.9 \\
\hline $1-128$ & $-1.775 E+01$ & 93.2 \\
\hline $8 a-139$ & $-1.199 E+02$ & 100.1 \\
\hline Dy -165 & 3. $95 B E+00$ & 9.9 \\
\hline$u-235$ & 4. $662 E+O O$ & 2.8 \\
\hline $\mathrm{Ga}-72$ & $-1.611 E+01$ & 79.8 \\
\hline$A S-76$ & 3. $110 E+O O$ & 12.4 \\
\hline$B r-82$ & $2.331 E+O O$ & 17.7 \\
\hline Mo- 99 & $4.620 E+O O$ & 14.1 \\
\hline$S b-122$ & 7. $286 E-01$ & 13.1 \\
\hline$L a-140$ & $2.532 E+O 1$ & 4. 1 \\
\hline$S m-153$ & $4.434 E+\infty O$ & 4.7 \\
\hline$Y b-175$ & $2.816 E+00$ & 6.1 \\
\hline Lu -177 & $5.136 E-01$ & 7.5 \\
\hline$w-187$ & $-1.639 E+00$ & 57.4 \\
\hline$A u-198$ & $-8.133 E-03$ & 100.2 \\
\hline Sc- 46 & $6.63 \backslash E+O O$ & 3.9 \\
\hline $\mathrm{Cr}-51$ & 1. $237 E+01$ & 10.7 \\
\hline $\mathrm{Fe}-59$ & 1. $170 E+O 4$ & 4.0 \\
\hline $\mathrm{Co}-6 \mathrm{O}$ & $2.985 E+00$ & 6.3 \\
\hline $2 n-65$ & $7.375 E+01$ & 13.5 \\
\hline $5 \theta-75$ & $-3.912 E+O O$ & 101.4 \\
\hline$R b-86$ & $2.804 \mathrm{E}+01$ & 17.5 \\
\hline $2 r-95$ & $-1.157 E+02$ & 100.3 \\
\hline $\mathrm{Ag}-110$ & $-1.759 E+00$ & 65.1 \\
\hline$S b-124$ & $5.607 E-01$ & 27.8 \\
\hline$C s-134$ & $2.590 E+O O$ & 10.0 \\
\hline$B a-131$ & $2.555 \mathrm{E}+\mathrm{O} 2$ & 14.3 \\
\hline $\mathrm{Ce}-141$ & $5.103 E+01$ & 4.2 \\
\hline$N d-147$ & $-9.686 E+\infty 0$ & 76.9 \\
\hline$E u-152$ & 6.891E-01 & 11.0 \\
\hline$T D-160$ & $6.838 \mathrm{E}-01$ & 18.0 \\
\hline Yb- 169 & 3. $252 E+00$ & 6.0 \\
\hline$L u-17 \%$ & $4.918 E-01$ & 6.3 \\
\hline$H f-18,1$ & $3.691 E+00$ & 4.8 \\
\hline$T a-182$ & $7.698 E-01$ & 15.8 \\
\hline $\mathrm{Hg}-203$ & $-6.625 E-01$ & 100.8 \\
\hline$T h-233$ & 1. $474 \mathrm{E}+\mathrm{O} 1$ & 3.3 \\
\hline
\end{tabular}

$\begin{array}{rr}3.2 B 7 E+O 3 & 3.5 \\ -1.643 E+O 3 & 93.4 \\ 3.424 E+O 4 & 4.5 \\ -6.331 E+O 1 & 74.1 \\ -3.499 E+O 3 & 97.1 \\ 1.623 E+O 4 & 11.2 \\ 2.638 E+O 3 & 9.2 \\ 3.728 E+O 1 & 8.8 \\ 4.702 E+O 2 & 3.5 \\ 2.154 E+O 2 & 100.8 \\ 2.708 E+O 2 & 100.3 \\ 2.484 E-O 1 & 24.1 \\ 1.767 E+O 1 & 93.3 \\ 2.618 E+O 2 & 13.5 \\ 3.245 E+O O & 9.8 \\ 2.615 E+O O & 2.8 \\ 1.898 E+O 1 & 23.8 \\ 3.707 E+O O & 11.6 \\ 7.933 E+O O & 10.3 \\ 5.71 O E+O O & 90.8 \\ 1.251 E+O O & 9.6 \\ 1.391 E+O 1 & 4.5 \\ 2.228 E+O O & 5.0 \\ 2.351 E+O O & 6.4 \\ 4.609 E-O 1 & 6.7 \\ 3.75 O E+O O & 18.4 \\ 8.785 E-O 3 & 100.2 \\ 5.955 E+O O & 3.9 \\ 2.127 E+O 1 & 6.5 \\ 5.4 B 3 E+O 3 & 4.4 \\ 2.469 E+O O & 6.5 \\ 5.712 E+O O & 88.2 \\ 3.109 E+O O & 26.1 \\ 3.462 E+O 1 & 12.7 \\ 1.524 E+O 2 & 71.5 \\ 1.785 E+O O & 65.1 \\ 1.032 E+O O & 27.6 \\ 2.777 E+O O & 8.9 \\ 3.044 E+O 2 & 13.2 \\ 2.927 E+O 1 & 4.5 \\ 9.006 E+O O & 77.6 \\ 4.263 E-O 1 & 13.2 \\ 5.757 E-O 1 & 18.1 \\ 2.764 E+O O & 6.4 \\ 4.542 E-O 1 & 6.0 \\ 3.132 E+O O & 4.9 \\ 1.267 E+O O & 12.0 \\ 6.430 E-O 1 & 100.8 \\ 1.098 E+O 1 & 3.4\end{array}$

\begin{tabular}{|c|c|}
\hline 62 & 4.0 \\
\hline $1.935 E+03$ & 25.7 \\
\hline 4. $157 E+04$ & 4.0 \\
\hline $4954 E+01$ & 76.5 \\
\hline $3302 E+03$ & \\
\hline $1.64 G E+04$ & 11.2 \\
\hline 2. $066 E+03$ & 10.7 \\
\hline A. $765 E+01$ & 7.8 \\
\hline A. $601 E+02$ & 3.5 \\
\hline $1.883 E+O 2$ & 100.8 \\
\hline $2.617 E+O 2$ & 100.3 \\
\hline $1.031 E-01$ & 31.2 \\
\hline $1.711 E+O 1$ & $93 \cdot 3$ \\
\hline 1. $182 E+02$ & 100.1 \\
\hline 2. $365 E+0 O$ & 13. 2 \\
\hline $2.722 E+O O$ & 2.8 \\
\hline $1.695 E+01$ & 80.2 \\
\hline A. $822 E+00$ & 12.2 \\
\hline A. $266 E+0 O$ & 13.8 \\
\hline 7. $343 E+00$ & 89.2 \\
\hline $6.929 E-01$ & 17.0 \\
\hline $1.741 E+01$ & 4.4 \\
\hline $2.131 E \div 00$ & 5.1 \\
\hline 2. $059 E+00$ & 6.8 \\
\hline $3.779 E-01$ & 8.8 \\
\hline A. $439 E+00$ & 21.5 \\
\hline 8. $763 E \cdot 03$ & 100.2 \\
\hline 5. $4 O A E+0 O$ & 3.9 \\
\hline $1.874 E+01$ & 8. 0 \\
\hline $6.687 E+03$ & A. 4 \\
\hline $3.670 E+00$ & 6.1 \\
\hline $2.254 E+01$ & 30.1 \\
\hline $2.634 E+00$ & 84.9 \\
\hline $2.468 \mathrm{E}+01$ & 17.1 \\
\hline $1.765 E+02$ & 71.7 \\
\hline $2.042 E+00$ & 64.9 \\
\hline $5.562 E-01$ & 82.9 \\
\hline $2.209 E+O O$ & 8.6 \\
\hline 1. $706 E+O 2$ & 24.5 \\
\hline 3. $139 E+O 1$ & 4.6 \\
\hline $1.536 \mathrm{~F}+01$ & 73.5 \\
\hline $3.662 \mathrm{E}-01$ & 15.9 \\
\hline $2.830 \mathrm{E}-01$ & 23.7 \\
\hline $2.985 E+00$ & 7.1 \\
\hline $4.249 E-01$ & 7.7 \\
\hline $2.549 E+00$ & 6.1 \\
\hline $7.599 E-01$ & 17.1 \\
\hline $.769 E-01$ & 100.8 \\
\hline $.969 E+00$ & 3.6 \\
\hline
\end{tabular}

1. $806 E+03$

4. $158 E+03$

6. $151 E+04$

5. $053 E+01$

5. $538 E+03$

. $343 E+04$

2. $420 E+03$

$316 E+01$

3. $797 E+02$

1. $784 E+O 2$

2. 306E +02

.775E-O1

1. $496 E+01$

2. $A 69 E+O 2$

4. $782 E+O O$

3. $900 E+00$

1. $.34 E+O 1$

3. $459 E+O O$

2. A2GE+OO

5. $128 E+00$

$1.167 \mathrm{E}+O$

2. $711 E+01$

4. $679 E+00$

$456 \mathrm{E}+0 \mathrm{O}$

5. $425 \mathrm{E}-01$

1. $784 E+O O$

$-7.204 \mathrm{E}-03$

. $6 E 6 E+O O$

. B 45E 01

2. $987 E+O O$

9. $253 \mathrm{E}+O O$

3. $250 E+O D$

.

4. $318 \mathrm{E}+0$

$-1.424 \mathrm{E}+00$

1. $057 E+00$

5. $712 \mathrm{E}+00$

1. $953 E+02$

5. $635 \mathrm{E}+\mathrm{O}$

2. $313 E+O 1$

6. $966 E-O 1$

7. $251 E-01$

3. $895 E+00$

6. $228 \mathrm{E}-01$

5. $524 E+O O$

1. $126 E+O O$

$-6.549 E-O$

1. $537 E+O$
3.9

3. 7

24.8

8.

3.5

00.8

100.3

93.2

13.4

80. 3

13.8

15.8

90.2 


$\begin{array}{lc}\text { FILE IO } & 6101170 \\ \text { SAMPLE } & 073 \\ \text { DUPLICATE } & 147 \\ \text { CLUSTER } & 10 \\ \text { TYPE } & \text { L }\end{array}$

6101180
074
119
05
$L$

6101190
075
153
10
1
6101200

076

05

ELEMENT

$\mathrm{Na}-24$

$\mathrm{Mg}-27$

A) - 28

Cl -38
$k-42$

$K-42$
$C a-49$

$\mathrm{TH}-51$

$V-52$
$M n-56$

$\mathrm{Mn}-56$
$\mathrm{Cu}-66$

$\mathrm{Cu}-66$
$\mathrm{Sr}-87$

In-116

I -128

Ba-139

Dy -165

$u-235$

$\mathrm{Ga}-72$

As - 76

$\mathrm{Br}-82$

Mo- 99

Sb- 122

La-140

$5 m-153$

YU -175
LU- 177

$w-187$

Au- 198

$\mathrm{SC}-46$

$\mathrm{Cr}-51$

$\mathrm{Fe}-59$

$\mathrm{CO}-6 \mathrm{O}$

$2 n-65$

Se- 75

Zr- 85

$\mathrm{Zr}-95$

Ag- 110

C5- 134

Ba-131

Ce-141

Nd-147

Eu- 152

Tb- 160

Yb-169

LU- 177

$\mathrm{Hf}-181$
$\mathrm{Ta}-182$

$\mathrm{Hg}-203$

$\mathrm{Hg}-2 \mathrm{O} 3$
$+\quad \mathrm{Th}-233$

\begin{tabular}{|c|c|}
\hline $2.826 E+03$ & \\
\hline $2.233 E+03$ & 26.7 \\
\hline $4.518 E+04$ & 4.0 \\
\hline 8. $050 E+01$ & 26.5 \\
\hline 3. $239 E+03$ & 97.1 \\
\hline $1.371 E+04$ & 11.5 \\
\hline $1.862 E+03$ & 11.5 \\
\hline $2.571 E+01$ & 10.3 \\
\hline 4. $107 E+02$ & 3.5 \\
\hline 1. $982 E+02$ & $100 . \mathrm{B}$ \\
\hline 2. $525 \mathrm{E}+\mathrm{O} 2$ & 100.3 \\
\hline 1. $315 E-01$ & 56.0 \\
\hline $1.650 E+01$ & 93.1 \\
\hline $2.083 E+02$ & 18.0 \\
\hline $3.359 E+O O$ & 11.4 \\
\hline $4.827 E+00$ & 2.8 \\
\hline $1.685 E+01$ & 25.5 \\
\hline 4. $395 E+00$ & 10.8 \\
\hline $4.322 E+00$ & 11.9 \\
\hline $1.406 E+00$ & 14.7 \\
\hline $5.260 E-01$ & 15.6 \\
\hline $2.064 E+01$ & 4.1 \\
\hline $3.444 E+00$ & 5.0 \\
\hline $2.505 E+00$ & 5.8 \\
\hline 4. $368 E-01$ & 7.0 \\
\hline $3.549 E+O O$ & 15.9 \\
\hline B. $054 E-03$ & 100.2 \\
\hline 5. $822 E+O O$ & 3.9 \\
\hline 1. $07 B E+01$ & 8.8 \\
\hline $9.434 E+03$ & 4.0 \\
\hline 2. $089 E+00$ & 6.7 \\
\hline $9.202 E+01$ & 11.0 \\
\hline 2. $172 \mathrm{E}+0 \mathrm{O}$ & 49.0 \\
\hline $2.041 E+01$ & 14.9 \\
\hline $1.456 E+O 2$ & 71.6 \\
\hline $1.693 E+00$ & 65.2 \\
\hline $5.354 E-01$ & 76.4 \\
\hline 1. $600 E+00$ & 11.0 \\
\hline $1.839 E+02$ & 16.2 \\
\hline $4.608 \mathrm{E}+01$ & 4.2 \\
\hline $3.878 E+0 O$ & 77.0 \\
\hline $4.934 E-01$ & 10.6 \\
\hline 1. $320 E-01$ & 17.7 \\
\hline $.821 E+00$ & 5.8 \\
\hline . 399E-01 & 6.1 \\
\hline $.350 E+00$ & 4. 1 \\
\hline $.103 E+00$ & 11.6 \\
\hline $.108 E-01$ & 100.8 \\
\hline
\end{tabular}

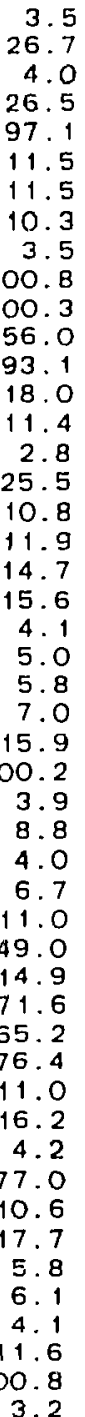

3.2
- $565 E+04$

6. $970 E+01$

$3.623 \mathrm{~F}+03$

1. $426 \mathrm{E}+04$

. $339 E+O 3$

3. $5 B 6 E+O 1$

. $263 \mathrm{E}+\mathrm{O} 2$

. $377 \mathrm{E}-\mathrm{O}$

$1.823 E+01$

3. $129 E+02$

. $849 E+O O$

3. $844 \mathrm{E}+\mathrm{OO}$

5. $182 E+O$

5. $088 E+00$

7. $868 E+00$

1. $076 \mathrm{E}+\mathrm{OO}$

3. $163 E+O 1$

4. $3 O O E+O O$

. $585 \mathrm{E}+00$

$.878 \mathrm{E}-01$

2. $469 E+O O$

$-1.072 \mathrm{E}-02$

6. $445 \mathrm{E}+0 \mathrm{O}$

1. $910 E+01$

1. $084 E+O 4$

3. $468 E+O 0$

3. $199 \mathrm{E}+O$

4. $712 \mathrm{E}+00$

3.762E+

2. $286 E+O O$

. $655 \mathrm{E}+0$

3. $655 E+00$

4. $173 E+02$

$6.871 \mathrm{E}+\mathrm{O}$

$-1.783 \mathrm{E}+01$

$7.656 \mathrm{E}-01$

7. $479 \mathrm{E}-01$

3. $982 E+00$

$6.016 \mathrm{E}-\mathrm{O}$

1. $136 \mathrm{E}+\mathrm{OO}$

$-8.882 E-01$

1. $229 \mathrm{E}+01$
3. 7

93.4

23.1

97.0

12.6
9.1

9.9

3.5
100.8

OO. 3

56.1

93.3

10.8
7.9

2. 8

79.6

12.0

10.4
88.9

13.3

5. 1

6.2

6.4

60.9
100.2

3. 9

4.1

6.4

39. 3

39.3

71.5

65.4

27.4

13.3

4.2
73.2

10.7

15.4

6.0
6.4

5.1
14.7

100.8 $.613 E+03$

$800 E+04$

4.0

.849E $+01 \quad 75.2$

.396E+03

$.319 E+04 \quad 13.1$

$2.282 E+01 \quad 13.8$

5.035E+02 3.5

. $045 E+02 \quad 100 . B$

$2.957 E+02 \quad 100.3$

$-1.401 E-01 \quad 56.3$

1. $9 A 4 E+01 \quad 93.2$

2. $091 \mathrm{E}+\mathrm{OO} \quad 16$.

1. $612 \mathrm{E}+\mathrm{OO} \quad 2 . \mathrm{B}$

. $584 \mathrm{E}+00$

17.5

2.893E+OO 16.5

361E-01 2O.2

$.435 E+O O \quad 5.1$

2. $171 \mathrm{E}+0 \mathrm{O}$

2. $986 \mathrm{E}+00$

4. $479 E-01$

$3.86 B E+00$

6. 507E-OJ

3. $970 E+00$

. $348 E+01$

4. $636 E+O O$

3. $110 E+O$

.

2. $903 E+00$

$129 \mathrm{E}+02$

$418 E+00$

$229 E-O 1$

$169 E+O O$

. $334 \mathrm{E}+\mathrm{O} 2$

$115 E+01$

. $842 E+O O$

2. 965E-O 1

2. BO6E-01

3. $158 \mathrm{E}+00$

5. $198 E-O 1$

2. $012 \mathrm{E}+0 \mathrm{O}$

3. $682 \mathrm{E}-01$

4. $985 \mathrm{E}+0 \mathrm{O}$
1. 109E +03

3. $713 \mathrm{E}+03$

4. $716 \mathrm{E}+04$

-4. $271 E+01$

$-3.223 E+O 3$

$216 E+04$

$473 E+03$

4. $536 \mathrm{E}+01$

5. $051 E+02$

$-1.836 E+02$

$-2.606 E+02$

1. $407 E-01$

$-1.153 E+O 2$

4. $961 \mathrm{E}+00$

3. $023 \mathrm{E}+00$

9. $521 \mathrm{E}+O D$

5. $250 E+O O$

2. $560 E+O O$

$-5.298 E+O O$

9. $4 \mathrm{~B} \mathrm{1E}-\mathrm{O} 1$

3. $192 \mathrm{E}+\mathrm{O} 1$

4. $795 E+00$

5. $315 E-01$

2. $6 O B E+O O$

7. $810 \mathrm{OE}-\mathrm{O} 3$

7. $282 \mathrm{E}+\mathrm{OO}$

2. $104 \mathrm{E}+\mathrm{O} 1$

$576 E+00$

$326 \mathrm{E}+\mathrm{OO}$

2. $132 E+O O$

$122 E+01$

1. $868 E+00$

. $522 E-01$

.

$780 E+01$

. $233 E+O 1$

8. $866 \mathrm{E}-01$

. $789 E+00$

4. $351 E+O O$

$.066 E-01$

$1.057 \mathrm{E}+01$
4.4

3.9

77.5

97.2

7.7

3. 5

100.3

25.3

93.3

8.8

2.8

23.3

9.1

90.6

10.0

4.7

1.8

5.9

100.2 
Na- 24

$\mathrm{Mg}-27$

A $1-28$

C $1-38$

$k-42$
$C a-49$

$\mathrm{T} i-51$

$v-52$

$\mathrm{Mn}-56$
$\mathrm{Cu}-66$

$\mathrm{Cu}-66$
$\mathrm{Sr}-87$

In- 116

I -128

Ba- 139

$D_{y}-165$

Ga- 72

$\mathrm{Ga}-72$

$\mathrm{As}-76$
$\mathrm{Br}-\mathrm{B}$

$\mathrm{Br}-82$

Sb- 122

La- 140

Sm- 153

$Y b-175$
$L U-177$

$W-187$

Au- 198

Sc- 46

$\mathrm{Cr}-51$

Co- 60

$2 n-65$

$2 n-65$

$\mathrm{Rb}-86$

$\mathrm{Zr}-95$

$\mathrm{Ag}-110$

Sb-124

Cs-134

Ba-131

$\mathrm{Ce}-141$
$\mathrm{Na}-147$

Eu-152

Tb- 160

Yb- 169

LU- 177

Hf -181

Ta-182

$\mathrm{Hg}-203$
$\mathrm{Th}-233$

\begin{tabular}{|c|c|}
\hline $6.564 \mathrm{E}$ & \\
\hline $3.723 \mathrm{E}+03$ & 12.6 \\
\hline $3.565 \mathrm{E}+04$ & 4.2 \\
\hline $3.878 E+01$ & 77.7 \\
\hline $3.125 E+03$ & 97.1 \\
\hline 1. $425 \mathrm{E}+04$ & 11.8 \\
\hline $1.523 E+03$ & 9.7 \\
\hline 4. $981 E+01$ & 7. \\
\hline $4.769 E+02$ & \\
\hline$-1.640 E+02$ & 100.8 \\
\hline$-2.413 E+02$ & 100.3 \\
\hline $1.044 E-01$ & 29.1 \\
\hline$-1.599 E+01$ & 93.3 \\
\hline$-9.512 E+01$ & 100.1 \\
\hline $3.484 E+O O$ & 9.2 \\
\hline $2.721 E+O O$ & \\
\hline$-8.209 E+\infty 0$ & 79.7 \\
\hline $4.635 E+O O$ & 10.6 \\
\hline $3.25 O E+O O$ & 11.4 \\
\hline$-6.44 O E+O O$ & 89.2 \\
\hline $5.556 E-01$ & 13.1 \\
\hline $1.792 E+O 1$ & 4. 1 \\
\hline $2.629 \mathrm{E}+00$ & 5.0 \\
\hline $2.401 E+00$ & 6.6 \\
\hline $4.014 E-01$ & 7.9 \\
\hline 2. $437 E+O O$ & 17.6 \\
\hline $7.607 E-03$ & 100.2 \\
\hline $5.517 E+O O$ & 3.9 \\
\hline $2.068 E+01$ & 8.2 \\
\hline $1.035 E+04$ & 4.1 \\
\hline $2.332 E+O O$ & 7.2 \\
\hline 4. $973 \mathrm{E}+01$ & 15.8 \\
\hline $2.858 E+00$ & 36.4 \\
\hline$\therefore .813 \mathrm{E}+01$ & 22.5 \\
\hline $1.815 \mathrm{E}+\mathrm{O} 2$ & 71.6 \\
\hline 2. $109 E+O O$ & 65.2 \\
\hline $6.574 \mathrm{E}-01$ & 79.7 \\
\hline $3.283 E+00$ & 8.3 \\
\hline $1.850 E+02$ & 53.6 \\
\hline $3.915 E+01$ & 4.8 \\
\hline $1.520 E+01$ & 73.6 \\
\hline $4.924 E-01$ & 13.8 \\
\hline $2.627 \mathrm{E}-01$ & 28.5 \\
\hline $2.700 E+O O$ & 7.1 \\
\hline $3.716 \mathrm{E}-01$ & 8.5 \\
\hline $2.300 E+O O$ & 6.4 \\
\hline 5. $391 E-01$ & 21.2 \\
\hline $7.948 \mathrm{E}-0.1$ & 100.8 \\
\hline $9.355 \mathrm{E}+00$ & 3.6 \\
\hline
\end{tabular}

1. $957 E+03$

$-1.463 E+03$

2. $809 E+04$

$5.054 E+O 1$

-3. $328 E+03$

1. $629 \mathrm{E}+04$

1. $578 E+03$

2. $255 E+01$

$5.097 E+02$

1. $882 \mathrm{E}+\mathrm{O} 2$

$2.660 \mathrm{O}+\mathrm{O} 2$

8. 297E-02

$-1.753 E+O 1$

2. $139 \mathrm{E}+\mathrm{OZ}$

2. $651 E+0 O$

3. $427 E+00$

7. $392 \mathrm{E}+\mathrm{OO}$

4. $739 \mathrm{E}+\mathrm{OO}$

5. $124 \mathrm{E}+\mathrm{OO}$

$-4.361 \mathrm{E}+00$

6. $335 \mathrm{E}-\mathrm{O}$

1. $316 \mathrm{E}+01$

2. $355 E+O O$

1. $953 \mathrm{E}+00$

3. $088 \mathrm{E}-0$

2. $315 \mathrm{E}+\mathrm{OO}$

$-6.415 E-03$

4. $303 \mathrm{E}+00$

8. $871 E+00$

4. $868 \mathrm{E}+03$

$1.03 B E+00$

$1.072 E+01$

$4.042 \mathrm{E}+00$

$-6.573 E+00$

$-1.140 E+02$

$-1324 E+O 0$

$-4.217 E-01$

2. $545 E+O 1$

7. $398 E+O O$

3. $045 E-01$

2. $953 \mathrm{E}-01$

3. $592 \mathrm{E}-\mathrm{O}$

. $133 \mathrm{E}+\mathrm{OO}$

. 4 EE-0 1

6. $446 E-O 1$

8. $847 \mathrm{E}+\mathrm{OO}$
3. 8

94.4

1.6
75.8

97.2

10.7

12.9

3.5
$100 . \mathrm{B}$

100.3

33.2

93.3

19.5

79.3

9.9

90.5

2.

4. 3

6.5

9.5

100.2

3.9

1.3

4.5

54.8

20.6

100.0

71.5

65.0
78.6

13.3

21.7

4.6

14.7

20.2

6.8

5.6

17.0
100.8
3. $386 \mathrm{E}+\mathrm{O}$

1. $324 \mathrm{E}+03$

3. $924 E+O 4$

8. $150 E+01$

2. $483 E+03$

.

4. 185E+01

2. $685 \mathrm{E}+\mathrm{O} 2$

- $.722 E+O$

$-1.961 \mathrm{E}+02$

1. $927 E-01$

1. $285 E+O$

3. $274 E+02$

3. $900 \mathrm{E}+\mathrm{OO}$

$1.006 \mathrm{E}+\mathrm{O}$

3. $771 \mathrm{E}+\mathrm{OO}$

$4.094 E+00$

1. $006 \mathrm{E}+0 \mathrm{D}$

2. $201 \mathrm{E}+\mathrm{O}$

3. $B O 5 E+O D$

2. $33 \mathrm{OE}+O \mathrm{OO}$

2. $889 \mathrm{E}-\mathrm{O}$

1. $938 E+00$

$5.902 E+00$

1. $720 \mathrm{E}+\mathrm{O}$

$6.698 \mathrm{E}+03$

3. $565 \mathrm{E}+00$

7. $329 E+01$

3. $147 E+00$

2. $690 \mathrm{E}+\mathrm{O} 1$

1. $342 \mathrm{E}+\mathrm{O} 2$

$-1.613 E+00$

$-4.266 E-01$

$2.845 E+00$

3. $267 E+O 2$

4. $891 E+0$

1. $805 E+01$

5. $818 \mathrm{E}-\mathrm{O}$

4.259E-O

$353 E-O$

3. $999 \mathrm{E}+00$

.

8. 589 E 01

$-5.800 E-O 1$
$1.229 E+O 1$
3.4

4.8

25.2

97.0

8.9

8.4

00.8

oo. 3

19.7

93.2

8. 1

2.8
79.2

10.5

10. 8

90.8

8.8

5. 1

6.3

24.7

100.2

3.9
6.8

5. 4

5.4
11.7

24.2

4.4

28.3

65.0

84.2
7.5

1.3

3.9
30.5

0.0

19.7

5.6

6.3

4.2

100.8

3. 3
1. $564 E+03$

2. $758 E+03$

4. $428 E+04$

. $719 E+O 1$

4. $317 E+03$

$5.341 \mathrm{E}+03$

1. $424 \mathrm{E}+03$

$857 E+01$

1. $123 E+02$

$-1.426 E+02$

1. $557 \mathrm{E}+\mathrm{O} 2$

1. $203 \mathrm{E}-01$

$-9.989 E+00$

$-7.407 \mathrm{E}+01$

6. $901 \mathrm{E}+00$

3. $805 E+00$

5. $175 \mathrm{E}+00$

- $9.420 E+O O$

$4.867 E-01$

5. $956 \mathrm{E}+01$

$5.633 \mathrm{E}+\mathrm{OO}$

4. $172 \mathrm{E}+\mathrm{OO}$

5. $747 \mathrm{E}-01$

$5.946 \mathrm{E}+00$

$-1.117 \mathrm{E}-02$

4. $804 E+O 0$

$1.874 E+O 1$

. $201 E+04$

6. $055 \mathrm{E}+0$

2. $621 \mathrm{E}+O 0$

$709 \mathrm{E}+01$

1. 297E+O2

- $259 E+00$

$-2.059 E+00$

$4.796 E-O 1$
$3.274 E+O O$
$1.713 E+O 2$

1. $096 \mathrm{E}+02$

4. $359 \mathrm{E}+01$

$1.007 \mathrm{E}+00$

1. $190 E+O O$

4. $344 \mathrm{E}+0 \mathrm{O}$

6. $373 \mathrm{E}-\mathrm{O}$

2. $588 \mathrm{E}+00$

7. 869E-O

8. $74 \mathrm{E}-\mathrm{O}$

9. $056 \mathrm{E}+00$

4. 1

4.0

20. 1

16.2

8.0

100.8

100.3

93.

100. 1

7.1 
FILE ID

SAMPLE

DUPL I CATE

CLUSTER
TYPE

6101250

OB1

10

L
6101260

OB2

10
6101270

083

10
6101280

084
151

05

ELEMENT

\begin{tabular}{|c|c|c|}
\hline $\mathrm{Na}-24$ & $3.684 \mathrm{E}+03$ & 3.4 \\
\hline $\mathrm{Mg}-27$ & $-1.536 E+03$ & 93. \\
\hline$A\}-28$ & $3.349 E+04$ & 4. \\
\hline C1- 38 & $-5.759 E+01$ & 73 \\
\hline$k-42$ & $4.096 E+03$ & 26 \\
\hline Ca- 49 & $1.383 E+04$ & \\
\hline & $1.593 E+03$ & 11. \\
\hline$v-52$ & 3. $334 \mathrm{E}+01$ & 10. \\
\hline$M n-56$ & $4.039 E+02$ & 3 \\
\hline Cu- 66 & $-1.979 E+O 2$ & 100. \\
\hline$S r-87$ & $-2.415 E+O 2$ & 100. \\
\hline In-116 & $-1.315 E-01$ & 55. \\
\hline$I-128$ & $-1.591 E+01$ & 93. \\
\hline$B a-139$ & $-9.665 E+01$ & 100 \\
\hline$D y-165$ & 4. $37 \mathrm{OE}+O O$ & \\
\hline$U-235$ & $3.596 \mathrm{E}+00$ & \\
\hline $\mathrm{Ga}-72$ & $-9.047 E+00$ & 79. \\
\hline As - 76 & $3.276 E+O O$ & 10. \\
\hline $\mathrm{Br}-82$ & $2.679 E+00$ & 13. \\
\hline MO- 99 & $-5.037 E+00$ & 90. \\
\hline$S b-122$ & $7.133 E-01$ & 12. \\
\hline La- 140 & $1.733 E+01$ & \\
\hline$S m-153$ & $3.396 E+00$ & 4. \\
\hline$Y b-175$ & $2.570 E+O O$ & 6. \\
\hline Lu- 177 & 3. $800 E-01$ & 7. \\
\hline$W-187$ & $2.414 E+\infty 0$ & 19. \\
\hline$A u-198$ & $-7.676 \mathrm{E}-03$ & 100. \\
\hline Sc- 46 & $5.219 E+O O$ & 3 \\
\hline$C r-51$ & $9.788 E+00$ & \\
\hline $\mathrm{Fe}-59$ & $6.819 E+03$ & 4. \\
\hline $\mathrm{CO}^{-} 6 \mathrm{O}$ & $1.723 E+O O$ & 7. \\
\hline$Z n-65$ & 4. $440 E+01$ & \\
\hline Se- 75 & $7.571 E+00$ & 37. \\
\hline$R b-86$ & $2.759 E+01$ & 13. \\
\hline$Z r-95$ & $-1.238 E+02$ & 71. \\
\hline$A g-110$ & $-1.454 E+00$ & 65. \\
\hline $\mathrm{Sb}-124$ & $9.752 E-01$ & 30. \\
\hline$C s-134$ & $2.226 E+O O$ & 8. \\
\hline $\mathrm{Ba}-131$ & $9.328 E+01$ & 23. \\
\hline $\mathrm{Ce}-141$ & $3.590 E+01$ & \\
\hline Nd -147 & $-7.653 E+O O$ & 76 . \\
\hline$E U-152$ & $5.174 \mathrm{E}-01$ & 10 \\
\hline$T b-i 60$ & $3.709 E-01$ & 21. \\
\hline$Y b-169$ & $2.790 E+O O$ & 5 \\
\hline Lu- 177 & $4.158 \mathrm{E}-01$ & 6. \\
\hline$H f-181$ & $3.215 E+\infty$ & 4. \\
\hline$T a-182$ & $7.643 \mathrm{E}-01$ & 16. \\
\hline $\mathrm{Hg}-203$ & $-5.346 E-01$ & 100. \\
\hline$T h-233$ & $1.028 E+01$ & 3. \\
\hline
\end{tabular}

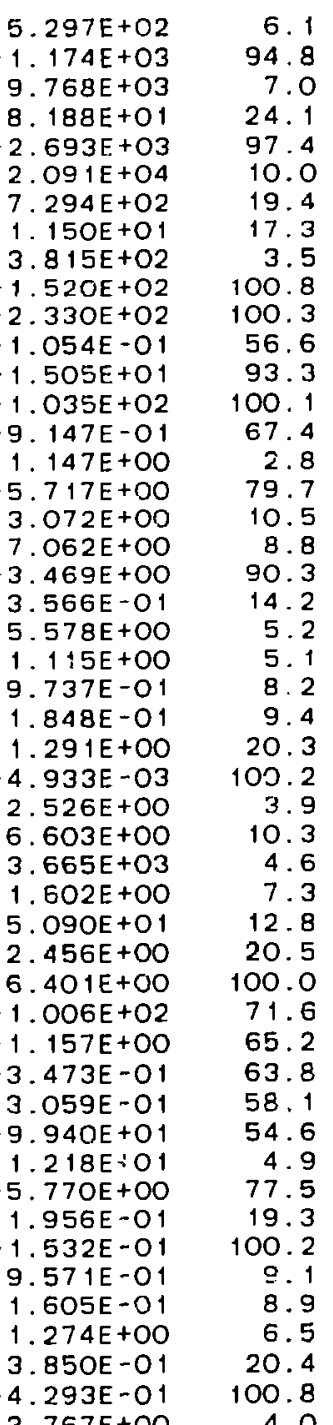

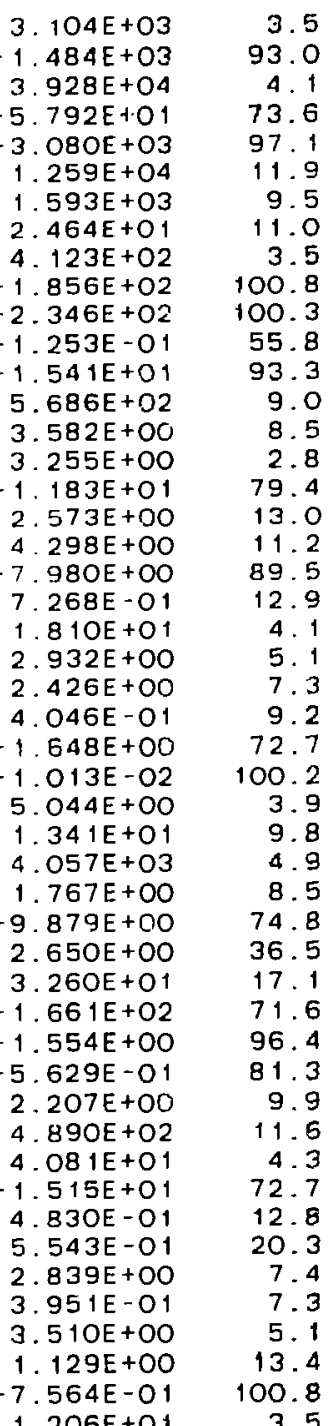

2. $617 \mathrm{E}+\mathrm{O}$

2. $176 \mathrm{E}+\mathrm{O3}$

2. $285 \mathrm{E}+04$

4. $635 \mathrm{E}+\mathrm{O}$

2. $576 E+03$

$.504 E+04$

$703 E+03$

$.175 E+01$

2. $702 E+02$

1. $664 E+02$

$2.016 \mathrm{E}+\mathrm{O} 2$

1. $096 E-01$

1. $311 E+01$

2. $622 E+02$

4. $501 E+O O$

2.088E+00

. $686 E+O O$

$795 E+00$

. $692 E+0 O$

$768 \mathrm{E}-01$

$.911 E+01$

3. $285 \mathrm{E}+00$

$548 E+00$

6. $655 E-01$

2. $357 \mathrm{E}+00$

$-7.112 \mathrm{E}-\mathrm{O} 3$

$6.927 \mathrm{E}+\mathrm{OO}$

2. $004 \mathrm{E}+01$

1. $002 E+04$

7. $228 E+00$

$5.520 E+01$

2. $713 E+O O$

2. $916 E+01$

1. $947 \mathrm{E}+02$

$-1.644 E+00$

4. $741 \mathrm{E}-01$

2. $195 \mathrm{E}+\mathrm{O} 2$

4. $295 E+01$

$8.273 \mathrm{E}+00$

7. $271 \mathrm{E}-01$

6. $360 E-O 1$

$5.480 E+O O$

$.600 E-01$

2. $748 E+O O$

$5.095 E-01$
$-5.949 E-01$

7. $292 \mathrm{E}+\mathrm{OO}$

18. 9

74.1

97.2

10.9

7.7

00.8 
ELEMENT

\begin{tabular}{|c|c|c|}
\hline $\mathrm{Na}-24$ & $3.045 E+03$ & 3. \\
\hline $\mathrm{Mg}-27$ & $-1.232 E+03$ & 93.1 \\
\hline A) - 28 & $2.254 E+O 4$ & \\
\hline C) $-3 B$ & $-5.051 E+01$ & 73. \\
\hline$k-42$ & $-2.460 E+03$ & 97.2 \\
\hline Ca- 49 & $1.449 E+04$ & 11.4 \\
\hline$T i-5 i$ & 1. $191 E+03$ & 13.1 \\
\hline$v-52$ & 2. $194 \mathrm{E}+01$ & 12.3 \\
\hline$M n-56$ & $2.523 E+O 2$ & 3.5 \\
\hline $\mathrm{Cu}-66$ & $-1.651 E+02$ & 100.8 \\
\hline$S r-87$ & $-1.911 E+02$ & 100 \\
\hline $1 n-116$ & $-1.042 E-01$ & 56 \\
\hline I -128 & $-1.260 E+01$ & 93 \\
\hline$B a-139$ & $3.100 E+02$ & 11.1 \\
\hline$D y-165$ & 3. $890 E+O O$ & 8.0 \\
\hline U -235 & 2. $583 E+O O$ & 2 \\
\hline$G a-72$ & $-1.043 E+C:$ & 79. \\
\hline $45-76$ & $4.470 E+O O$ & 9. \\
\hline $81-82$ & $6.585 E+0 O$ & \\
\hline Mo- 99 & $-5.448 E+00$ & 90.8 \\
\hline$S b-12.2$ & $9.274 E-01$ & 9.9 \\
\hline$L a-140$ & $1.264 E+01$ & \\
\hline$S m-153$ & $2.863 \mathrm{E}+00$ & 4.8 \\
\hline$Y b-175$ & $3.279 E+00$ & \\
\hline$L u-177$ & $5.526 \mathrm{E}-01$ & \\
\hline$w-187$ & $2.504 \mathrm{E}+00$ & 19.0 \\
\hline$A U-198$ & $-8.54 G E-03$ & 100.2 \\
\hline Sc- 46 & $5.010 E+O 0$ & 3.9 \\
\hline$C r-51$ & 9. $163 \mathrm{E}+00$ & 10 \\
\hline $\mathrm{Fe}-59$ & 5. $384 E+03$ & 4. \\
\hline $\mathrm{CO}-6 \mathrm{O}$ & 2. $164 E+O O$ & 6. \\
\hline $2 n-65$ & $-9.758 E+00$ & 68. \\
\hline $5 e-75$ & $1.827 E+00$ & 30. \\
\hline$R b-86$ & $1.778 \mathrm{E}+01$ & 15.7 \\
\hline $2 r-95$ & $-1.337 E+02$ & 71.7 \\
\hline $\mathrm{Ag}-110$ & $-1.570 E+0 O$ & 64. \\
\hline $5 b-124$ & $-5.742 E-01$ & 67. \\
\hline$C s-134$ & $7.702 \mathrm{E}-01$ & 17. \\
\hline$B a-131$ & $3.429 E+O 2$ & 10. \\
\hline$C e-141$ & $2.575 E+01$ & 4. \\
\hline No- 147 & $-7.823 E+00$ & 77. \\
\hline$E u-152$ & 4. 203E-01 & 11. \\
\hline$T b-160$ & $5.44 \mathrm{OE}-01$ & 17. \\
\hline$Y b-169$ & 3. $609 \mathrm{E}+00$ & 4. \\
\hline$L u-177$ & 5. $305 \mathrm{E}-01$ & 5. \\
\hline$H f-181$ & $2.411 E+O O$ & 5 . \\
\hline$T a-182$ & 5. $435 E-01$ & 24 \\
\hline $\mathrm{Hg}-203$ & $-5.582 E-01$ & 100. \\
\hline$T h-233$ & $7.734 E+00$ & 3. \\
\hline
\end{tabular}

\begin{tabular}{|c|c|}
\hline 2. $843 \mathrm{E}+03$ & 3.5 \\
\hline $1.475 E+03$ & 93.9 \\
\hline 3. $198 E+04$ & 4.4 \\
\hline $1.429 E+02$ & 14.5 \\
\hline 3. OB $5 E+O 3$ & 97.2 \\
\hline 1. $36 O E+O 4$ & 12.5 \\
\hline $1.525 E+03$ & 11.4 \\
\hline 2. $495 E+01$ & 11.5 \\
\hline 3. $788 \mathrm{E}+02$ & 3.5 \\
\hline $1.931 E+02$ & 100.8 \\
\hline $2.502 \mathrm{E}+\mathrm{O} 2$ & 100.3 \\
\hline $1.279 E-01$ & 56.1 \\
\hline $1.632 \mathrm{E}+01$ & 93.2 \\
\hline $2.65 d \mathrm{E}+\mathrm{O} 2$ & 19.2 \\
\hline $4.341 E+O O$ & 10.0 \\
\hline 2. $561 \mathrm{E}+\mathrm{OO}$ & 2.8 \\
\hline 1. $279 E+01$ & 79.2 \\
\hline $5.311 E+O O$ & 10.8 \\
\hline 8. $481 E+O O$ & 9.7 \\
\hline $8.564 \mathrm{E}+00$ & 89.4 \\
\hline $7.817 E-01$ & 20.0 \\
\hline 2. $342 E+01$ & 4.0 \\
\hline $3.628 E+00$ & 4.8 \\
\hline $2.769 E+O O$ & 7.1 \\
\hline $3.948 E-01$ & 9.7 \\
\hline 1. $584 \mathrm{E}+O O$ & 59.5 \\
\hline $1.085 E-02$ & 130.2 \\
\hline 4. $710 E+O O$ & 3.9 \\
\hline B. $000 E+00$ & 12.6 \\
\hline $6.859 E+03$ & 4.4 \\
\hline 1. $649 E+O O$ & 8.8 \\
\hline 3. $129 \mathrm{E}+01$ & 25.4 \\
\hline $3.260 E+O O$ & $\$ 00.0$ \\
\hline $4.753 E+01$ & 12.7 \\
\hline $1.755 E+02$ & 71.5 \\
\hline $2.005 E+00$ & 64.9 \\
\hline $6.001 E-01$ & B1.6 \\
\hline 3. $238 E+00$ & 9.0 \\
\hline 1. $749 E+02$ & 22.2 \\
\hline 5. $157 E+01$ & 4.4 \\
\hline $1.596 E+01$ & 72.3 \\
\hline $6.670 E-01$ & 13. 1 \\
\hline $7.057 E-01$ & 16.0 \\
\hline 3. $245 E+00$ & 6.7 \\
\hline $4.554 E-01$ & 7.0 \\
\hline $4.964 E+O O$ & 4.5 \\
\hline $5.491 E-01$ & 22.7 \\
\hline 8. $002 E-01$ & 100.8 \\
\hline $3.491 E+00$ & 3.7 \\
\hline
\end{tabular}

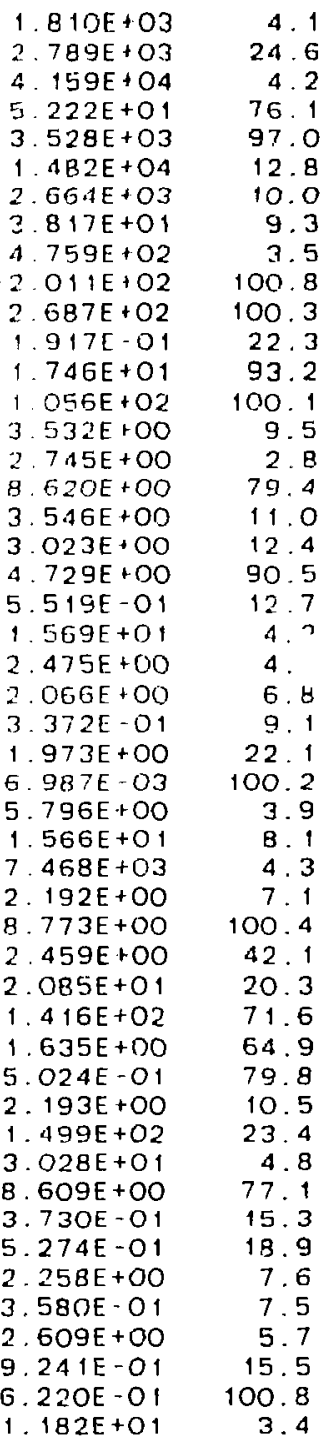

\begin{tabular}{|c|c|}
\hline $3.014 E+03$ & 3.4 \\
\hline $3.649 E+03$ & 15.9 \\
\hline $6.326 E+04$ & 3.6 \\
\hline $5.3 B 1 E+0$ & 74.0 \\
\hline $7.872 \mathrm{E}+03$ & 17.4 \\
\hline 1. $199 \mathrm{E}+04$ & 124 \\
\hline $2.273 E+03$ & 8.7 \\
\hline 4. $106 \mathrm{E}+01$ & 7.3 \\
\hline $3.623 \mathrm{E}+\mathrm{O} 2$ & 3.5 \\
\hline 1. $867 \mathrm{E}+\mathrm{O} 2$ & 100.8 \\
\hline $2.291 E+.02$ & 100.3 \\
\hline 1. $492 E-01$ & 26.0 \\
\hline 1. $483 E+01$ & 93.3 \\
\hline 4. $4 O B E+O 2$ & 9.9 \\
\hline 4. $362 E+00$ & 8.9 \\
\hline 4. $756 E+00$ & 2.8 \\
\hline $1.725 E+01$ & 18.5 \\
\hline 3. $852 E+O O$ & 10.6 \\
\hline $1.479 E+00$ & 15.0 \\
\hline $5.866 E+00$ & 90.8 \\
\hline $7.573 E-01$ & 11.6 \\
\hline $2.911 E+O 1$ & 3.8 \\
\hline 4. $\triangle B B E+O O$ & 4.9 \\
\hline $3.941 E+00$ & 4.9 \\
\hline $6.753 \mathrm{E}-01$ & 5.6 \\
\hline $3.811 E+C O$ & 16.0 \\
\hline $8.861 E-03$ & 100.2 \\
\hline $7.928 E+00$ & 3.8 \\
\hline 2. $488 \mathrm{E}+01$ & 6.2 \\
\hline 1. $876 E+O 4$ & 3.8 \\
\hline $4.921 E+00$ & 4.9 \\
\hline 1. $216 E+O 2$ & 10.4 \\
\hline $2.024 E+O O$ & 50.7 \\
\hline $5.711 E+O 1$ & 8.6 \\
\hline 1. $316 E+O 2$ & 100.3 \\
\hline $1.927 E+00$ & 65.3 \\
\hline $5.383 E-01$ & 83.7 \\
\hline 6. $344 \mathrm{E}+00$ & 5.6 \\
\hline 4. $659 E+02$ & 9.3 \\
\hline $5.964 \mathrm{E}+01$ & 3.8 \\
\hline $9.492 \mathrm{E}+00$ & 77.6 \\
\hline $7.692 \mathrm{E}-01$ & B. 4 \\
\hline $6.474 \mathrm{E}-01$ & 15.0 \\
\hline $4.778 \mathrm{E}+00$ & 4.1 \\
\hline $6.669 E-01$ & 4.9 \\
\hline $4.752 E+O O$ & 4.0 \\
\hline 1. $283 E+00$ & 11.1 \\
\hline $6.868 E-01$ & 100.8 \\
\hline 1. $497 E+01$ & \\
\hline
\end{tabular}




$\begin{array}{lc}\text { FILE ID } & 6101330 \\ \text { SAMPLE } & 089 \\ \text { DUPLICATE } & 117 \\ \text { CLUSTER } & 10 \\ \text { TYFE } & L\end{array}$

\section{ELEMENT}

$\mathrm{Na}-24$

Mg- 27

A $1-28$

Cl -38

$k-42$

$\mathrm{Ca}-49$

$T i-5 i$

$V-52$
Mn- 56

Cu- 66

$5 r-87$

In- 116

Ba-139

Dy -165

$\mathrm{U}-235$
$\mathrm{Ga}-72$

$\mathrm{As}-76$

$\mathrm{Br}-82$

Mo- 99

Sb- 122

$5 m-153$

Yb- 175

Lu- 177

-187
$A u-198$

Ac- 46

SC- 46

Cr 59

Co- 60

n- 65

Se- 75

$\mathrm{Rb}-86$
$2 \mathrm{r}-95$

Ag- 110

SD- 124

Cs-134

$B a-131$

Ce-141

Eu-152

Tb- 160

Yb- 169

Lu- 177

$\mathrm{Ta}-182$

\& $\begin{aligned} \mathrm{Hg}-203 \\ \mathrm{Th}-233\end{aligned}$
$-1.427 E+03$

$1.451 \mathrm{E}+04$

$5.955 E+01$

$-3.090 E+03$

1. $730 E+04$

9. $384 \mathrm{E}+02$

2. $037 \mathrm{E}+01$

$-1.908 E+02$

- $525 \mathrm{E}+\mathrm{O} 2$

$-2.525 E+02$

$-1.257 E-01$

2. $477 \mathrm{E}+\mathrm{O} 2$

4. $867 \mathrm{E}+0 \mathrm{O}$

2. $364 \mathrm{E}+0 \mathrm{O}$

1. $229 E+O 1$

3. $619 E+O O$

5. $064 E+00$

$7.866 \mathrm{E}+00$

1. $248 \mathrm{E}+00$

$7.876 \mathrm{E}+\mathrm{OO}$

2. $296 \mathrm{E}+\mathrm{OO}$

4. $076 \mathrm{E}+\mathrm{OO}$

6. $47 \mathrm{OE}-\mathrm{O} 1$

$3.322 \mathrm{E}+\mathrm{OO}$

$9.699 \mathrm{E}-03$

$4.780 E+00$

$59+E+O O$

$2.791 E+03$

. $179 E+00$

2. $077 E+01$

$-2.385 E+O O$

1. $009 E+01$
$2.686 E+02$

.

$-7.048 E-01$

6. 593E-01

3. $237 E+O 2$

1. $647 \mathrm{E}+01$

1. $509 E+01$
$3.627 E-01$

$6.845 \mathrm{E}-01$

4. $960 \mathrm{O}+0 \mathrm{O}$

$.778 E-01$

$3.015 E+00$

$-4.694 \mathrm{E}-01$

$7.525 E-O 1$
$5.310 E+00$
3.6

93.6

6.

97.3

10.8

6.1

12. 7

3.5

100.3

56.2

93.3

2.9

7.9
2.8

78.8

13.0

10.8

89.3

8.7

5.2
4.9

5.4

6.1

6.1
18.6

100.

3.9

12.6

11.1

35.6

98.6

100.0

64.8

68.2

27.7

16.7

73.2

15.7

17.2

4.9

5.8

5.8
39.8

100.8

4.3
2. $198 \mathrm{E}+03$

2. $44 O E+O 3$

3. $797 E+04$

5. $357 \mathrm{E}+01$

-3. $339 E+03$

1. $420 E+04$

4. $201 \mathrm{E}+0$

4. $445 \mathrm{E}+\mathrm{O} 2$

$-1.953 E+O 2$

$-2.613 E+O 2$

1. $400 E-01$

1. $727 \mathrm{E}+01$

2. $326 \mathrm{E}+\mathrm{O} 2$

1. $.040 E+00$

2. $882 \mathrm{E}+00$

$8.617 E+O O$

3. $364 \mathrm{E}+\mathrm{OO}$

2. $102 E+O O$

$-4.465 E+0 O$

$1.333 \mathrm{E}+01$

1. $883 \mathrm{E}+\mathrm{OO}$

1. $464 E+O O$

2. $550 \mathrm{O}-\mathrm{O} 1$

$2.568 E+O O$

$5.726 E+O O$

2. $326 \mathrm{E}+\mathrm{O} 1$

$6.885 \mathrm{E}+03$

1. $960 E+O O$

. $477 E+01$

2. $061 E+00$

2. $1 \mathrm{~B} 4 \mathrm{E}+\mathrm{O} 1$

-1. $332 E+02$

$-1.532 E+00$

$-4.617 \mathrm{E}-01$

3. $839 E+00$

-1. $167 \mathrm{E}+\mathrm{O} 2$

2. $416 \mathrm{E}+01$

3. $034 \mathrm{E}-01$

4. $177 \mathrm{E}-01$

4. $177 E-01$
1. $363 E+O O$

2. $735 E-01$

$2.881 \mathrm{E}+00$

1. $221 E+00$

$-5.673 E-01$
$8.473 E+00$
3. 7

22.2
4.2

75.2

97.1

10.9

10.2

8. 5
3. 5
00.8

100.8

23.1
93.2
19.7

19.7

67.3
2.8
79.5
11.2

11.2

18.7

90.7

4.3

5.2
8.0
10.3

0.3

100.

3.

3.9

6.4

7.2

9.

39.4

71.6

64.8

81.1

63.1

4.9

77.3

14.0

19.9

(1.

5.2

11.7

100.8
3.5
1. $562 \mathrm{E}+03$

1. $576 \mathrm{E}+\mathrm{O} 3$

2. $191 \mathrm{E}+04$

1. $598 \mathrm{E}+02$

$-3.601 E+03$

1. $553 E+04$

1. $35 \mathrm{OE}+03$

3. $498 \mathrm{E}+01$

$2.079 E+02$

2. $962 E+O 2$

$1.412 \mathrm{E}-\mathrm{O}$

1. $969 \mathrm{E}+01$

1. $173 E+02$

6. $556 \mathrm{E}+\mathrm{OO}$

2. $870 E+O 0$

$6.959 \mathrm{E}+\mathrm{OO}$

$4.427 \mathrm{E}+00$

$6.287 E+O 0$

1. $026 E+00$

4. $954 E+01$

4. $985 \mathrm{E}+00$

7. $\because 12 \mathrm{E}-01$

2. $707 E+0 O$

9. $002 \mathrm{E}-\mathrm{O} 3$

6. $141 E+O O$

1. $458 \mathrm{E}+04$

3. $516 E+O O$

B. $964 \mathrm{E}+01$

4. $009 E+00$

2. $571 E+01$

2. $028 E+O O$

$5.698 \mathrm{E}-\mathrm{O}$

2. $162 \mathrm{E}+\mathrm{OO}$

1. $301 \mathrm{E}+\mathrm{O} 2$

$6.27 \mathrm{BE}+01$

$-1.027 \mathrm{E}+01$

8. $793 \mathrm{E}-\mathrm{O} 1$

9. 157E-01

5. $235 E+00$

3. $806 \mathrm{E}+00$

$6.196 E-01$

$-7.473 E-01$

8. $224 E+D O$

4.4

94.0

5.4

97.3

12.3

13.2

3. 5

100.3

56.3

00. 1

2.8
79.5

9.2

11.1

90.4

3.9

4.8 


$\begin{array}{ccc}\circ & \text { FILE ID } & 6101370 \\ \text { SAMPLE } & 093 \\ \text { DUPLICATE } & 143 \\ \text { CLUSTER } & 10 \\ \text { TYPE } & \text { L }\end{array}$

6101380
094
139
06
$T$

$\mathrm{O} 6$
6101390

149

G8
6101400

096

02

ELEMENT

$\mathrm{Na}-24$

$\mathrm{Mg}-27$

Al- 28

C1 - 38

$K=42$

Ca- 49

Ti- $5 \dagger$

$v-52$

Mn- 56

$\mathrm{Cu}-66$

In-116

I -128

Ba- 139

Dy -165

$\mathrm{U}-235$
$\mathrm{Ga}-72$

As - 76

$\mathrm{Br}-82$

MO- 99

Sb- 122
La- 140

$5 m-153$

Yb- 175

Lu-177

Au- 198

Sc- 46

$\mathrm{Cr}-51$

$\mathrm{Fe}-59$

$\mathrm{CO}-6 \mathrm{O}$

$2 n-65$

$\mathrm{Se}-75$
$\mathrm{Rb}-86$

Zr- 95

Ag- 110

$\mathrm{Sb}-124$
$\mathrm{CS}-134$

Ba- 131

$\mathrm{Ce}-141$

EU- 152

rb- 169

Yb- 169
Lu- 177

Hf -181

$T a-182$

$\mathrm{Hg}-2 \mathrm{O3}$
$\mathrm{Th}-233$

$\begin{array}{rr}2.424 E+O 3 & 3.8 \\ -1.531 E+O 3 & 93.3 \\ 3.68 O E+O 4 & 4.4 \\ -5.340 E+O+ & 75.1 \\ -3.357 E+O 3 & 97.0 \\ 1.425 E+O 4 & 13.0 \\ 2.462 E+O 3 & 9.1 \\ 4.61 O E+O 1 & 9.2 \\ 3.68 O E+O 2 & 3.5 \\ -1.991 E+O 2 & 100.8 \\ -2.447 E+O 2 & 100.3 \\ 1.676 E-O 1 & 20.8 \\ -1.621 E+O 1 & 93.2 \\ 2.652 E+O 2 & 13.0 \\ 3.891 E+O O & B .9 \\ 4.163 E+O O & 2.8 \\ -9.866 E+O O & 79.9 \\ 3.679 E+O O & 11.5 \\ 5.562 E+O O & 10.3 \\ -5.431 E+O O & 90.6 \\ 5.913 E-O 1 & 13.5 \\ 2.055 E+O 1 & 4.1 \\ 3.344 E+O O & 5.0 \\ 2.22 O E+O O & 7.4 \\ 2.962 E-O 1 & 10.6 \\ 1.663 E+O O & 25.8 \\ -8.105 E-O 3 & 100.2 \\ 5.654 E+O O & 3.9 \\ 2.473 E+O 1 & 7.0 \\ 5.328 E+O 3 & 4.5 \\ 2.020 E+O O & 7.5 \\ 4.135 E+O 1 & 18.2 \\ 3.433 E+O O & 26.3 \\ -8.470 E+O O & 100.0 \\ -1.254 E+O 2 & 97.0 \\ -1.673 E+O O & 64.9 \\ -4.919 E-01 & 85.3 \\ 2.009 E+O O & 11.7 \\ 2.317 E+O 2 & 15.4 \\ 4.309 E+O 1 & 4.2 \\ -9.226 E+O O & 76.4 \\ 5.246 E-O 1 & 11.7 \\ 5.248 E-O 1 & 20.5 \\ 2.659 E+O O & 7.6 \\ 3.944 E-O 1 & 7.0 \\ 4.121 E+O O & 4.7 \\ 1.098 E+O O & 14.2 \\ -6.34 O E-O 1 & 100.8 \\ 1.493 E+O 1 & 3.4\end{array}$

7. $B 13 E+O 3$

1. $172 E+O 5$

9. $956 E+03$

$101 E+O A$

$.548 E+03$

7. $352 E+00$

2. $159 E+02$

2. $855 \mathrm{E}+\mathrm{O} 2$

5. $303 \mathrm{E}+\mathrm{O} 2$

$-1.673 \mathrm{E}-01$

$-1.753 E+O$

9. $319 E+02$

4. $62 \mathrm{BE}+0 \mathrm{O}$

2. $017 E+O 1$

1. $870 E+O 0$

$-1.799 E+O O$

$-9.373 E+O O$

1. $346 E+O 0$

5. $227 E+01$

$3.211 E+O O$

4. $174 \mathrm{E}-\mathrm{O}$

2. $428 \mathrm{E}+\mathrm{OO}$

$-1.429 E-02$

3. $586 E+O D$

3. $581 E+O O$

1. $125 E+O A$

1. $125 E+O A$

1. $260 \mathrm{OE}+02$

$2.641 E+00$

$6.693 E+01$

1. $292 \mathrm{E}+\mathrm{O} 2$

1. $596 \mathrm{E}+00$

2. $571 \mathrm{E}+\mathrm{OO}$

8. $737 \mathrm{E}+\mathrm{O} 2$

1. $071 \mathrm{E}+02$

4. $652 \mathrm{E}+\mathrm{O} 1$

4. 191E-D

9. $156 \mathrm{E}-01$

3. $204 E+O O$

3. $572 \mathrm{E}-\mathrm{O} 1$

5. $700 E+D O$

3. $697 E+0 O$

$-7.380 E-01$
$4.804 E+01$

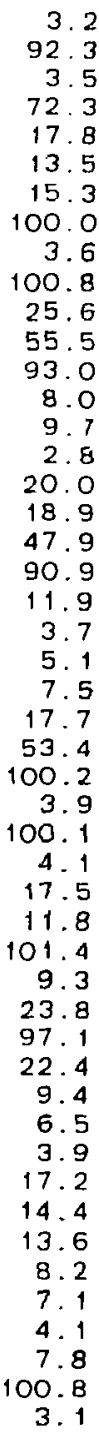

6. $549 E+O 3$

1. $194 \mathrm{E}+04$

9. $749 E+04$

7. $319 E+01$

8. $463 \mathrm{E}+03$

. $227 \mathrm{E}+04$

2. $326 E+03$

3. $324 \mathrm{E}+\mathrm{O}$

2. $453 E+02$

2. $416 \mathrm{E}+\mathrm{O} 2$

4. $330 E+C 2$

1. $451 \mathrm{E}-\mathrm{O}$

1. $531 E+01$

A. $.079 E+02$

$2.872 E+00$

$+.823 E+01$

3. $976 \mathrm{E}+0 \mathrm{O}$

1. $699 \mathrm{E}+00$

1. $066 \mathrm{E}+\mathrm{O}$

1. $390 E+O D$

2. $230 E+D 1$

2. $696 E+00$

1. $682 E+00$

2. $647 \mathrm{E}-\mathrm{O}$

$2341 E+O O$
$-1.351 E-O 2$

7. $164 \mathrm{E}+0 \mathrm{O}$

1. $592 \mathrm{E}+\mathrm{O}$

1. $925 \mathrm{E}+04$

3. $131 \mathrm{E}+00$

1. $997 \mathrm{E}+02$

2. $816 \mathrm{E}+\mathrm{OO}$

5. $464 \mathrm{E}+\mathrm{O} 1$

1. $525 E+02$

$-2.526 E+O 0$

1. $187 E+O O$

7. $655 E+00$

2. $990 E+02$

4. $880 E+O$

16E $-O$

3. $316 E-O$

$-9.658 E-O$

2. $750 E-01$

$6.565 E+00$

1. $818 E+O 0$

1. $018 E+O O$
3. $949 E+O 1$ f. $368 \mathrm{E}+04$

3. $158 \mathrm{E}+03$

8. $923 \mathrm{E}+04$

7. $214 \mathrm{E}+\mathrm{O} 2$

3. $204 \mathrm{E}+\mathrm{O} 4$

7. $740 E+03$

$6.853 E+02$

1. $180 E+01$

4. $339 E+02$

3. $342 E+0$ ?

3. $245 E+O 2$

3. $696 E-01$

-2. $+27 E+01$

$-1.515 E+0$ ?

$181 E+O$

T. $532 E+01$

$-1.84 O E+01$

1. $766 \mathrm{E}+\mathrm{O}$

$-1.656 \mathrm{E}+\mathrm{OO}$

- 8. $706 E+00$

1. $829 \mathrm{E}+00$

3. $058 \mathrm{BE}+01$

1. $177 \mathrm{E}+\mathrm{O} 1$

7. $948 E+O O$

$.056 \mathrm{E}+00$

2. $542 \mathrm{E}+\mathrm{OO}$

1. $325 E-02$

2. $455 E+O O$

3. $244 \mathrm{E}+00$

1. $084 E+O$

1. $498 \mathrm{EE}+0 \mathrm{O}$

B I6E+O2

. B16E+O2

2. $760 \mathrm{OE}+02$

$2.076 E+02$

2. $076 \mathrm{E}+\mathrm{O} 2$

2. $\triangle B \mathrm{BE}+00$

$1.038 E+01$

1. $919 E+O$

B. $098 E+01$

4. $857 E+01$

975E-O

1. $843 \mathrm{E}+\mathrm{OO}$

8. $707 \mathrm{E}+00$

1. $091 E+O 0$

1. $396 E+O$

$4.567 E+00$

6. 559E-O

5. $462 \mathrm{E}+\mathrm{O}^{1}$
3.0

3.6

B. 2

17.9

96.0

21.0

3.5

100.8
100.3

22.4

too. 1

7.4

2.8

8.1

47.9

90.8

10.3

$4 \cdot 9$

6.2

100.2

4.0

4.1

4.1

10. 4

101. 4

18.2

99.4

17.0

5.2
13.1

3. 9

13.3

8.4

4.0

4.0

3. 4

8.8

100.8
3.0 


$\begin{array}{lc}\text { FILE ID } & 6101410 \\ \text { SAMPLE } & 097 \\ \text { DUPLICATE } & 114 \\ \text { CLUSTER } & O B \\ \text { TYPE } & T\end{array}$

ELEMENT

$\mathrm{Na}-24$

$\mathrm{Mg}-27$

Al- 28

C $1-38$

$k-42$

$\mathrm{Ca}-49$
$\mathrm{~T} i-51$

$v-52$

$\mathrm{Mn}-56$

$\begin{array}{ll}\mathrm{Cu}- & 66 \\ \mathrm{Sr}- & 87\end{array}$

$\mathrm{Sr}-87$
$\mathrm{In}-116$

In -116
I -128

$\mathrm{Ba}-139$

Dy- 165

$U-235$

Ga- 72

$\mathrm{Br}-\mathrm{B2}$

$\mathrm{Br}-\mathrm{B2}$
$\mathrm{MO}-99$

Sb-122

$\mathrm{L} a-140$
$\mathrm{Sm}-153$

$\mathrm{Sm}-153$
$\mathrm{Yb}-175$

Yb-175

$\mathrm{LU}-177$
$W-187$

Au- 198

Sc- 46

$\mathrm{Cr}-51$
$\mathrm{Fe}-59$

$\mathrm{CO}-60$

$\mathrm{Zn}-65$

$\mathrm{Se}-75$

Rb- 86

$2 r-95$
$A g-110$

st- 124

$5 b-124$

Ba-131

$\mathrm{Ce}-141$

Nd- 147

EU- 152
Tb- 160

Yb- 169

LU- 177

Hf -181

$\mathrm{T}-182$
$\mathrm{Hg}-203$

$\triangleq \quad \mathrm{Th}-233$
CLUSTER

OB

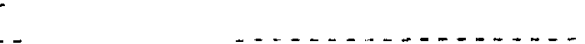

6. $847 \mathrm{E}+\mathrm{O} 4$
7. $267 \mathrm{E}+\mathrm{O} 2$

4. $699 E+03$

1. $177 \mathrm{E}+\mathrm{O} 3$

4. $466 \mathrm{E}+00$

. $997 E+02$

3. $33 O E+O 2$

- $992 \mathrm{E}-\mathrm{O}$

2. $179 \mathrm{E}+01$

4. $776 \mathrm{E}+\mathrm{O} 2$

6. $354 \mathrm{E}+\mathrm{OO}$

9. $416 \mathrm{E}+\mathrm{OO}$

2. $284 \mathrm{E}+\mathrm{O}$

3. $215 \mathrm{E}+00$

. $212 E+00$

3. $797 \mathrm{E}+\mathrm{OO}$

. $903 E-O$

6. $378 \mathrm{E}+00$

$5.015 E+00$

9. $738 \mathrm{E}-\mathrm{O}$

- 2 . $610 E+O O$

1. $613 \mathrm{E}-\mathrm{O} 2$

3. $885 \mathrm{E}+0 \mathrm{O}$

$1.097 \mathrm{E}+0$

1. $022 \mathrm{E}+\mathrm{O} 4$

2. $102 E+O O$

1. $465 E+O 2$

$-1.892 E+00$

$4.845 E+02$

1. $829 E+02$

$1.703 E+00$

$-5.023 E-0$

$1.021 \mathrm{E}+01$

2. $947 E+O 2$

8. $368 \mathrm{E}+01$

1. $216 E+O$

8. $345 E-O t$

$6.561 E+O O$

8. $789 \mathrm{E}-\mathrm{O}$

7. $282 \mathrm{E}+\mathrm{OO}$

2. $363 E+O O$

3. $557 \mathrm{E}+\mathrm{O}^{1}$
3.0

3. 9

8.0
7.7

20.3

17.5

3.5

100.8

(5).

93.2

13.4

8.2
2.8

78.4

15. 2

24.0
14.7

17.9

3.8
5.0

5. 4

2

56.2

100.2
3.9

10.8

4.1
7.3

10.1
101.4

4.4

21.5

65.5

5.2

5.2
12.6

3.9

74.0

11.7

4.2
4.9

3. 8

8.7
100.8

3.1
6101420

098

06
6101430

099
129

0
6101440

100

$\mathrm{OB}$

\begin{tabular}{|c|c|}
\hline $2.193 E+03$ & 3.7 \\
\hline$-1.318 E+03$ & 93.6 \\
\hline $3.665 E+04$ & 4.2 \\
\hline$-4.878 E+01$ & 74.9 \\
\hline $5.275 E+03$ & 24.1 \\
\hline $1.624 E+04$ & 10.9 \\
\hline 1. $496 E+03$ & 13.0 \\
\hline $2.084 E+01$ & 11.0 \\
\hline 3. $935 E+O 2$ & 3. 5 \\
\hline$-1.760 E+02$ & 100.8 \\
\hline $3.948 E+02$ & 24.5 \\
\hline $1.433 \mathrm{E}-01$ & 24.7 \\
\hline$-1.519 E+01$ & 93.2 \\
\hline $2.705 E+02$ & 13.1 \\
\hline 4. $394 E+00$ & 8. 3 \\
\hline $4.313 E+00$ & 2.8 \\
\hline $9.095 E+00$ & 79.3 \\
\hline $3.673 E+00$ & 11.0 \\
\hline $3.708 E+00$ & 12.1 \\
\hline$-4.689 E+00$ & 90.5 \\
\hline B. $381 \mathrm{E}-01$ & 12.5 \\
\hline 1. $781 E+01$ & 4. 1 \\
\hline $3.363 E+00$ & 4.8 \\
\hline $2.926 E+O D$ & 5.7 \\
\hline $4.435 \mathrm{E}-01$ & 7.2 \\
\hline $4.619 E+00$ & 14. 7 \\
\hline$-6.803 E-03$ & 100.2 \\
\hline $4.427 E+00$ & 3.9 \\
\hline $9.501 E+00$ & 10.4 \\
\hline $4.762 \mathrm{E}+\mathrm{O} 3$ & 4. 5 \\
\hline $1.479 E+00$ & 7.9 \\
\hline 3. $141 E+01$ & 19.8 \\
\hline $3.001 E+00$ & 26.9 \\
\hline 2. $128 \mathrm{E}+01$ & 13.6 \\
\hline $1.369 E+O 2$ & 24.3 \\
\hline$-1.336 E+00$ & 64.8 \\
\hline 8. 189E-01 & 29.2 \\
\hline 2. $142 E+O O$ & 8. 7 \\
\hline $3.317 \mathrm{E}+\mathrm{O} 2$ & 17.3 \\
\hline $3.787 E+01$ & 4.2 \\
\hline$-8.036 E+00$ & 75.8 \\
\hline 3. $583 \mathrm{E}-01$ & 13.2 \\
\hline $6.889 E-01$ & 15.2 \\
\hline 3. $126 \mathrm{E}+00$ & 5.8 \\
\hline $5.031 \mathrm{E}-01$ & 6.2 \\
\hline $3.602 E+00$ & 4. 7 \\
\hline $1.019 E+O O$ & 13.0 \\
\hline$-5.217 \mathrm{E}-01$ & 100.8 \\
\hline $1.378 E+01$ & 3.3 \\
\hline
\end{tabular}

$\begin{array}{rr}7.185 E+O 3 & 3.1 \\ 5.778 E+O 3 & 13.9 \\ 1.017 E+O 5 & 3.4 \\ 1.356 E+O 2 & 25.4 \\ 1.303 E+O 4 & 13.9 \\ 1.043 E+O 4 & 13.2 \\ 1.352 E+O 3 & 14.4 \\ 9.141 E+O O & 24.3 \\ 4.227 E+O 2 & 3.5 \\ 2.586 E+O 2 & 100.8 \\ 2.809 E+O 2 & 100.3 \\ 1.892 E-O 1 & 24.9 \\ 1.837 E+O 1 & 93.3 \\ 4.793 E+O 2 & 11.0 \\ 6.963 E+O O & 8.2 \\ 1.247 E+O 1 & 2.8 \\ 2.272 E+O 1 & 21.4 \\ 8.164 E+O O & 9.1 \\ 1.521 E+O O & 47.7 \\ 2.083 E+O O & 9.8 \\ 1.423 E+O O & 8.8 \\ 4.485 E+O 1 & 3.7 \\ 7.513 E+O O & 5.1 \\ 4.486 E+O O & 5.4 \\ 6.693 E-O 1 & 8.5 \\ 2.573 E+O O & 22.2 \\ 1.194 E-O 2 & 100.2 \\ 4.667 E+O O & 3.9 \\ 7.719 E+O O & 16.4 \\ 8.067 E+O 3 & 4.1 \\ 1.363 E+O O & 8.5 \\ 1.584 E+O 2 & 9.9 \\ -9.213 E-O 1 & 101.4 \\ 9.711 E+O 1 & 6.9 \\ 1.722 E+O 2 & 17.8 \\ 1.614 E+O O & 65.1 \\ 1.501 E+O O & 21.8 \\ 4.796 E+O O & 6.2 \\ 5.402 E+O 2 & 7.6 \\ 1.024 E+O 2 & 3.8 \\ 4.351 E+O 1 & 16.7 \\ 4.252 E-O 1 & 12.6 \\ 9.839 E-O 1 & 11.2 \\ 5.197 E+O O & 4.7 \\ 6.994 E-O 1 & 5.1 \\ 9.022 E+O O & 3.5 \\ 3.79 O E+O O & 7.1 \\ 6.699 E-O 1 & 100.8 \\ 4.588 E+O 1 & 3.0\end{array}$

3. 1

3.4

72.0

12. 2

7.3

3.6

100.8

27.6

6.7

9.3

2. 8

30.6

47.8

12.1

3. 7

12.7

16.0

54.5

100.2

21.5

101.4

8.4

20.6

24.4

7.8

6.9
3.9

20. 1

13.4

12.1

9. 1

9.4

100.8
3.3

2. $109 E+O$

5. 4

3. 2

4.3

o. 8

24. 9

1.0

2. 8

21.4

47. 7

B. 8

5. 1

8. 5

00. 2

6. 4

8.5

9.9
01.4

6.9

65.1

6.2

3. 8

2. 6

4.7

3. 5

3.0
3.0 


\section{ELEMENT}

$\mathrm{Na}-24$

Mg- 27

C. -38

$K-42$

Ca- 49

$T 1-51$
$V-52$

$M n-56$

$\mathrm{Cu}-66$

Sr- 87

In -116
I -128

Ba- 139

Dy -165

$U-235$
$\mathrm{Ga}-72$

As - 76

$\mathrm{Br}-82$

Mo- 99

Sb- 122

La-140

$5 m-15$

Yo- 175
Lu- 177

$W-187$

$4 U-198$
$S C-46$

Cr- 51

$\mathrm{Fe}-59$

$\mathrm{CO}-60$

$2 n-65$

$\mathrm{Se}-75$

$\mathrm{Rb}-86$

Zr -95

Ag- 110

So-124

Cs -134

Ba-131

Nul 147

Eu-152

Tb- 160

Yb- 169

LU- 177

Ia -182

$\mathrm{Hg}-2 \mathrm{O} 3$

Th-233

\begin{tabular}{|c|c|}
\hline $1.010 E+04$ & 3.0 \\
\hline$-2.13 O E+03$ & 91.7 \\
\hline 8. $214 E+04$ & 3.6 \\
\hline d. $273 E+O 2$ & 9.4 \\
\hline $2.493 \mathrm{E}+04$ & 9.1 \\
\hline $4.7+4 E+O 3$ & 20.4 \\
\hline $1.719 \mathrm{E}+03$ & 10.3 \\
\hline 2. $716 E+01$ & 13.1 \\
\hline 3. $197 E+O 2$ & 3.5 \\
\hline$-2.731 E+O 2$ & 100.8 \\
\hline$-2.62 O E+O 2$ & 100.3 \\
\hline$-1.58 \Delta E-01$ & 55.9 \\
\hline$-1.719 E+O 1$ & 93.1 \\
\hline $2.602 \mathrm{E}+\mathrm{O} 2$ & 13.9 \\
\hline $5.829 E+00$ & 7.8 \\
\hline $9.000 E+00$ & 2.8 \\
\hline 2. $234 \mathrm{E}+01$ & 78.7 \\
\hline $3.411 E+00$ & 14.0 \\
\hline $2.014 E+O O$ & 47.7 \\
\hline $1.262 E+01$ & 89.6 \\
\hline 1. $248 E+00$ & 12.8 \\
\hline $3.981 E+01$ & 3.8 \\
\hline 5. $961 \mathrm{E}+00$ & 4.8 \\
\hline 3. $520 E+O O$ & 6.9 \\
\hline $6.578 E-01$ & 9.1 \\
\hline $2.829 E+00$ & 49.0 \\
\hline 1. $583 \mathrm{E}-02$ & 100.2 \\
\hline 4. $509 E+00$ & 3.9 \\
\hline 1. $069 E+01$ & 14.5 \\
\hline 1. $396 E+04$ & 4.0 \\
\hline $2.711 E+O O$ & 7.0 \\
\hline $4.045 E+01$ & 21.9 \\
\hline $5.672 E+00$ & 92.0 \\
\hline $1.878 E+02$ & 6.1 \\
\hline $1.867 \mathrm{E}+02$ & 71.6 \\
\hline $3.822 \mathrm{E}+0 O$ & 26.9 \\
\hline 1. $506 E+D 0$ & 27.2 \\
\hline $9.677 E+00$ & 5.6 \\
\hline $2.657 \mathrm{E}+02$ & 16.1 \\
\hline 8.891E+01 & 4.1 \\
\hline 4. $536 E+01$ & 21.3 \\
\hline $6.072 \mathrm{E} \cdot 01$ & 12.6 \\
\hline $1.121 E+O O$ & 12.3 \\
\hline $4.721 E+00$ & 6.1 \\
\hline $6.349 E-01$ & 6.4 \\
\hline $5.583 E+00$ & 4.4 \\
\hline 2. $267 E+O O$ & 9.5 \\
\hline 8. $900 E-01$ & 100.8 \\
\hline $2.828 E+01$ & 3.2 \\
\hline
\end{tabular}

\begin{tabular}{|c|c|}
\hline $5.574 \mathrm{E}+03$ & 3.2 \\
\hline $5.083 f+03$ & 18.3 \\
\hline $9.021 E+04$ & 3.6 \\
\hline $3.864 E+02$ & 10.3 \\
\hline 1. $693 E+04$ & 12.1 \\
\hline $7.618 E+03$ & 17.2 \\
\hline $6.75 \mathrm{GE}+02$ & 95.6 \\
\hline $7.858 E+\infty 0$ & 100.0 \\
\hline $5.395 E+02$ & 3.5 \\
\hline $2.852 E+02$ & 100.8 \\
\hline $3.252 \mathrm{E}+02$ & 100.3 \\
\hline $1.827 \mathrm{E}-01$ & 55.8 \\
\hline 2. $143 \mathrm{E}+\mathrm{O} 1$ & 93.2 \\
\hline 1. $485 E+02$ & 100.1 \\
\hline $3.917 E+00$ & 11.0 \\
\hline 1. $102 \mathrm{E}+01$ & 2.8 \\
\hline 1. $4 A S E+O H$ & 79.2 \\
\hline $7.906 E+00$ & 9.4 \\
\hline 1. $046 E+00$ & 100.3 \\
\hline $5.648 E+O O$ & 10.9 \\
\hline 1. $164 \mathrm{E}+00$ & 10.5 \\
\hline 3. $195 \mathrm{E}+01$ & 3.9 \\
\hline 5. $178 E+00$ & 5.0 \\
\hline $2.787 E+00$ & 7.7 \\
\hline $3.893 E-01$ & 15.3 \\
\hline $1.976 E+00$ & 26.6 \\
\hline $1.057 \mathrm{E}-02$ & 100.2 \\
\hline $5.215 E+00$ & 3.9 \\
\hline $3.912 E+00$ & 26.1 \\
\hline $6.466 E+03$ & 4.4 \\
\hline$G .023 E-01$ & 14.2 \\
\hline $7.015 E+01$ & 13.5 \\
\hline $2.600 E+00$ & 100.9 \\
\hline $1.825 E+02$ & 5.8 \\
\hline 1. $237 E+O 2$ & 20.9 \\
\hline $1.625 E+00$ & 65.0 \\
\hline 1. $367 E+O O$ & 23.3 \\
\hline 4. $319 E+00$ & 7.2 \\
\hline $7.760 E+01$ & 26.1 \\
\hline $7.099 E+01$ & 3.9 \\
\hline $1.096 E+01$ & 72.7 \\
\hline $4.623 E-01$ & 12.9 \\
\hline 5. $170 E-01$ & 17.5 \\
\hline $2.783 E+00$ & 6.5 \\
\hline $4.724 E-01$ & 6.8 \\
\hline $4.705 E+00$ & 4.3 \\
\hline $212 E+00$ & 9. 9 \\
\hline $467 E-01$ & 100.8 \\
\hline $135 E+01$ & \\
\hline
\end{tabular}

3. $831 \mathrm{E}+03$

$9.212 \mathrm{E}+04$
$5.641 \mathrm{E}+02$

2. $446 E+O 4$

. $863 E+03$

1. $010 E+03$

A $587 \mathrm{E}+02$

2. $986 E+02$

3. OBOE +02

1. B81E-O 1

2. $014 E+01$

$251 E+00$

$074 E+0$

2.001E+01

. $383 E+O 0$

2. $22 E+O 0$

1. $516 E+00$

2. $930 E+01$

4. $260 E+D O$

2. $503 E+O O$

. $716 E-01$

2. $337 E+00$

1. $361 E-O 2$
5. $349 E+O O$

2. $558 \mathrm{E}+00$

5. $615 \mathrm{E}+03$

5. $689 \mathrm{E}-01$

1. $37 \mathrm{AE}+\mathrm{O} 2$

2. $940 E+O O$

2. $394 E+O 2$

. $580 E+02$

1. $463 E+O O$

$.474 E+00$

$.915 E+00$

6. $766 E+01$

1. $224 E+01$

$6.963 E-01$

2. $745 E+00$

$.887 E-01$

$115 E+00$
$996 E+00$

$-7.284 \mathrm{E}-01$

3. $299 \mathrm{E}+01$
3.1
18.5

9.0

9.0
10.2
19.8

17.5

3. 5

100.8

100.3

55.4
93.2

100.

2. 8

9.

28.

9.2
11.2

3.9

5.

B. 1
18.3

24.6

100.2

100. 1

4.5

15.4

0.4

101.4

88.2

96.6

22.7

22.7

5.8
19.4

4.0

71.0

14.1
17.5

7.9

7.6
7. $93 \mathrm{BE}+03$ 4. $911 E+03$

1. $405 E+05$

$296 E+O$

7. $258 \mathrm{E}+03$

1. $265 \mathrm{E}+04$

1. $597 \mathrm{E}+03$

g. $077 \mathrm{E}+00$

$5.491 \mathrm{E}+02$

. $838 E+02$

3. $150 E+02$

1. $801 E-01$

2. $060 E+O$

1. $430 E+O 2$

3. $424 E+O O$

1. $243 E+O$

2. O91E+O

4. $373 E+O 0$

1. $772 E+O 0$

1. $166 E+00$

1. $506 E+00$

4. $728 E+01$

$5.481 E+00$

$2129 \mathrm{E}+\mathrm{OO}$

$2.621 E-O 1$

2. $905 E+00$

1. $408 \mathrm{E}-\mathrm{O} 2$

4. $036 E+00$

-2. $751 E+O O$

5. $679 E+03$

$1.001 E+00$

1. $015 \mathrm{E}+02$

3. $160 \mathrm{O}+00$

4. $417 t+01$

$-1.0911+02$

$-1.828 E+00$

$1.889 \mathrm{~F}+00$

1. $894 \mathrm{E}+00$

3 $97 \mathrm{BE}+\mathrm{O} 2$

1.008: + 02

$-2.048 E+01$

4. 486E-01

7. 666 [: -01

1. $829 \mathrm{E}+00$

2. $284 E-01$

$7.466 E+00$

4. $6960+00$

-8. $284 E-01$

5. $313 E+D 1$
3.1

3. 3

73.4

12.8

14.5

100.0

100.8

100.3

55.6

93.2

11.8

2. 8

19.8

12.7

47.7

9. 7

5.7

12.2

46.6

28.7
100.2

100. 1

11.2

11.4

101.4

11.3
100.3

65.1

20.7

10.7

10.8

65.8

13.7

16.6

21.0

12.6

3.9

100.8 


\section{FILE ID \\ SAMPLE \\ OUPLICATE \\ CLUSTER \\ TYPE}

ELEMENT

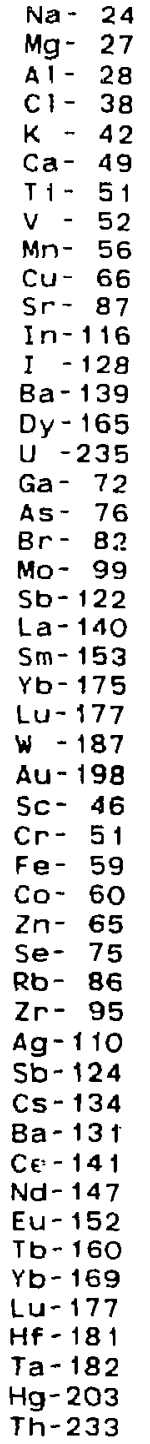

$\mathrm{Na}-24$
$\mathrm{Mg}-27$

A 1 - 28

K -42

$1-51$

n- 56

-87
$r-87$

a-139

$y-165$

a- 72

$-122$

a -140

b- 175

$-187$

$-5$

$\mathrm{CO}^{-} 50$

$2 n-65$

8 - 86

$\mathrm{Ag}-110$

U-152

Pb-169

Hf -18

$\mathrm{Hg}-203$

की $\mathrm{Th}-233$
6101490

105

125
6101500

106

09
6101510

107
6101520

108

10

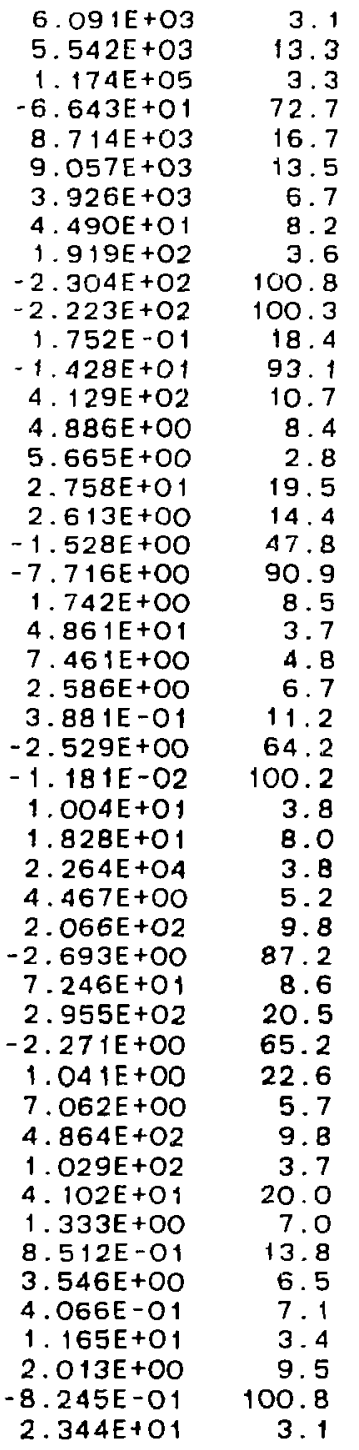

\begin{tabular}{|c|c|}
\hline $9.803 E+03$ & 3.0 \\
\hline 5. $178 E+03$ & 13.7 \\
\hline $8.689 E+04$ & 3.5 \\
\hline$-7.647 E+01$ & 71.7 \\
\hline 1. $176 \mathrm{E}+\mathrm{O} 4$ & 14.9 \\
\hline $1.042 E+04$ & 12.7 \\
\hline $2.117 E+03$ & 8.9 \\
\hline $2.351 E+01$ & 10.4 \\
\hline $2.457 E+02$ & 3.6 \\
\hline$-2.463 E+O 2$ & 100.8 \\
\hline $5.096 E+02$ & 20.2 \\
\hline $1.7 \mid 1 E-01$ & \\
\hline$-1.453 E+O 1$ & 93.0 \\
\hline $1.292 E+03$ & 6.2 \\
\hline 6. $153 E+00$ & 7.5 \\
\hline $9.645 E+00$ & 2.8 \\
\hline $2.206 E+01$ & 78.5 \\
\hline $6.099 E+00$ & 10.6 \\
\hline $2.386 E+0 O$ & 21.9 \\
\hline $3.781 E+00$ & 17.7 \\
\hline 1. $056 E+00$ & 14.7 \\
\hline $3.763 E+01$ & 3.8 \\
\hline 5. $190 E+00$ & 5.6 \\
\hline $3.790 E+O O$ & 6.7 \\
\hline $5.530 E-01$ & 10.4 \\
\hline$-2.965 E+00$ & 55.4 \\
\hline$-1.463 E-02$ & 100.2 \\
\hline $5.412 E+O O$ & 3.9 \\
\hline $7.982 E+O O$ & 15.6 \\
\hline 1. $176 E+04$ & \\
\hline 1. $825 E+00$ & 7.9 \\
\hline 6. $802 E+01$ & 14.0 \\
\hline $1.023 E+00$ & 101.4 \\
\hline $4.744 E+01$ & 11.4 \\
\hline $9.010 E+01$ & 32.5 \\
\hline 1. $620 E+O O$ & 97.3 \\
\hline $9.430 E-01$ & 29.5 \\
\hline $2.592 \mathrm{E}+00$ & 9.5 \\
\hline 1. $219 E+O 3$ & \\
\hline $7.860 E+01$ & 4.1 \\
\hline 4. $490 E+01$ & 24.5 \\
\hline 7. $353 E-01$ & 10.2 \\
\hline $5.318 E-01$ & 18.6 \\
\hline $3.900 E+00$ & 6.5 \\
\hline $5.540 E-01$ & 6.5 \\
\hline $6.653 E+O O$ & 4.0 \\
\hline $2.082 E+00$ & 10.2 \\
\hline $8.397 E-01$ & 100.8 \\
\hline $3.024 E+01$ & 3.1 \\
\hline
\end{tabular}

3. $258 \mathrm{E}+03$

1. $696 \mathrm{E}+03$

94.5

4. $989 \mathrm{E}+03$

3.3
10.1

3. 4

1. $253 E+05$

4. $572 E+03 \quad 96.8$

9. $613 \mathrm{E}+03 \quad 15.7$

17.2
$-7.969 E+00 \quad 100.0$

$5.869 E+02 \quad 3.5$

$-2.638 \mathrm{E}+02 \quad 100.8$

$1.100 E+02$
$-110 E-01$ 5.7

55.7

2. $365 \mathrm{E}+02 \quad 17.5$

. $673 E+00 \quad 8.8$

1. $264 E+01 \quad 2.8$

2.583E+01

$-1.190 E+O O \quad 47.7$

$-8.675 \mathrm{E}+00 \quad 53.4$

$\begin{array}{ll}2.485 E+O O & 6.4 \\ 4.560 E+01 & 3.7\end{array}$

$6.499 \mathrm{E}+0 \mathrm{O} \quad 4.9$

2. $238 E+00 \quad 9.3$

$-1.655 E+00 \quad 49.1$

$-9.749 E-03$

3. $625 \mathrm{E}+0 \mathrm{OO}$

2. $112 \mathrm{E}+00$

100.2

100.1

4.4

$5.956 \mathrm{E}-01 \quad 11.8$

$6.322 \mathrm{E}+01 \quad 14.1$

2.374E+01 16.7

2.

18.1

96.7

$\begin{array}{ll}2.496 \mathrm{E}+00 & 16.1 \\ 1.497 \mathrm{E}+O O & 11.6\end{array}$

$1.650 \mathrm{E}+02 \quad 12.3$

$9.921 \mathrm{E}+01 \quad 3.8$

$2.997 \mathrm{E}+01 \quad 23.0$

4.543E-01 12.2

$4.740 E-O 1 \quad 16.3$

$3.364 \mathrm{E}-01 \quad 8.1$

$6.468 E+00 \quad 3.9$

$5.825 E-01 \quad 100.8$

3.0

3. 1

3. $531 \mathrm{E}+04$

$6.485 \mathrm{E}+01$

5. $292 \mathrm{E}+03$

$1.577 E+04$

2. $358 \mathrm{E}+\mathrm{O} 3$

3. $435 \mathrm{E}+\mathrm{O} 1$

4. $612 \mathrm{E}+02$

$-2.219 E+02$

$-2.891 E+02$

$-1.480 \mathrm{E}-01$

1. $892 E+O$

2. $224 \mathrm{E}+\mathrm{O} 2$

2. $649 E+00$

2. $564 \mathrm{E}+0 \mathrm{O}$

1. $194 \mathrm{E}+0$

3. $209 E+00$

$-5.166 E+O 0$

9. $317 \mathrm{OE}-\mathrm{O} 1$

2. $133 E+O 0$

2. $133 E+00$

3. $483 \mathrm{E}-01$

3. $167 \mathrm{E}+\mathrm{OO}$

$-7.863 E-03$

5. $391 \mathrm{E}+\mathrm{OO}$

1. $940 E+O$

4. $784 \mathrm{E}+03$

2. $247 \mathrm{E}+\mathrm{OO}$

$4.478 E+O$

4. $478 \mathrm{E}+01$

3. $065 \mathrm{E}+0$

- $1361 \mathrm{E}+02$

$-1.573 E+00$

1. $224 E+00$

2. $153 \mathrm{E}+\mathrm{OO}$

2. $348 \mathrm{E}+\mathrm{O} 2$

2. $672 \mathrm{E}+01$

$-8.542 E+L O$

3. $990 E-01$

3. $303 \mathrm{E}-01$

1. $973 E+00$

3. 298E-O1

2. $736 E+O O$

1. $118 \mathrm{E}+0 \mathrm{O}$

$-5.897 E-01$
$9.910 E+O O$

4.5

74.0

29.0

11.2

9.5

3.5

100.3

56.0

93. 2

1B. 1

13.8

2.8
78.8

13.2

10.5

90.8 


$\begin{array}{lc}\text { FILE ID } & 6101530 \\ \text { SAMPLE } & 109 \\ \text { DUPLICATE } & 076 \\ \text { CLUSTER } & 05 \\ \text { TYPE } & L\end{array}$

6101540

110
085

10

6101550
111
062
03

6101560

112

05

\section{ELEMENT}

$\mathrm{Na}-24$

$\mathrm{Mg}^{-} 27$

A1 - 28

$C 1-38$

Ca- 49

$\mathrm{Ca}-49$

$T i-51$
$V-52$

Mn- 56

Cu- $6 \mathrm{G}$

In -116
I -128

Ba-139

$D y-165$

$u-235$

Ga- 72

$\mathrm{Br}-8$

Mo- 99

Sb-122

La- 140

$5 m-153$

Yb- 175

Lu-177

w- 187

Au- 198

$\mathrm{Sc}-46$
$\mathrm{Cr}-51$

$\mathrm{Fe}-59$

CO- 60

$\mathrm{Zn}-65$

Se- 75

Rb- 86

$\mathrm{Zr}-95$

Ag- 110

Sb- 124

$\mathrm{S} b-124$
$\mathrm{Cs}-134$

Ca -131

Ce-141

Eu-152

Tb- 160

$\mathrm{Yb}-169$

LU -177

Hf -181

Ta- 182

$\mathrm{Hg}-203$

\begin{tabular}{|c|c|}
\hline 1. $120 E+03$ & \\
\hline 3. $134 E+03$ & 15 \\
\hline $4.572 E+04$ & \\
\hline$-4.44 O E+01$ & \\
\hline$-3.399 E+03$ & \\
\hline 1. $283 E+04$ & 2 \\
\hline $2.204 E+03$ & \\
\hline 4. $288 \mathrm{E}+01$ & \\
\hline $5.064 E+02$ & \\
\hline$-1.855 E+02$ & \\
\hline$-2.575 E+02$ & 100 \\
\hline$-1.294 E-01$ & \\
\hline$-1.680 E+01$ & 93. \\
\hline$-1.023 E+02$ & \\
\hline $5.507 E+00$ & \\
\hline $2.936 E+0 O$ & \\
\hline 1. $.825 E+01$ & 25 . \\
\hline $4.670 E+O O$ & 10. \\
\hline 4. $287 E+00$ & 10. \\
\hline$-5.512 E+O O$ & 90. \\
\hline $8.935 E-01$ & 10. \\
\hline $3.236 \mathrm{E}+\mathrm{O} 1$ & 3. \\
\hline $4.807 E+00$ & . \\
\hline 4. O4OE +OO & 5. \\
\hline $6.269 E-01$ & 5. \\
\hline $2.537 \mathrm{E}+00$ & 19. \\
\hline$-7.796 \mathrm{E}-03$ & 100. \\
\hline 7.169 & 3. \\
\hline $1.740 E+01$ & 7. \\
\hline $1.237 E+04$ & 3. \\
\hline 2.742 & 6. \\
\hline $5.323 E+C$ & 14. \\
\hline 1. $47 \mathrm{BE}+\mathrm{C}$ & 52. \\
\hline $2.679 E+C$ & 14. \\
\hline 2. $256 E+O 2$ & 29. \\
\hline$-1.887 E+00$ & 65. \\
\hline$-5.451 E-01$ & BO. \\
\hline 4. $626 E+00$ & 6. \\
\hline $8.850 E+01$ & 26. \\
\hline & 4. \\
\hline $3.549 E+01$ & 22 . \\
\hline & \\
\hline $6.816 \mathrm{E}-\mathrm{Or}$ & 14. \\
\hline $3.943 E+00$ & 4. \\
\hline $6.083 E-01$ & \\
\hline 4. $463 E+00$ & \\
\hline $6.911 \mathrm{E}-01$ & \\
\hline$-6.652 E-01$ & \\
\hline
\end{tabular}

\begin{tabular}{|c|c|}
\hline $3.243 E+03$ & 3.5 \\
\hline 1. $161 E+03$ & 30.9 \\
\hline $\begin{array}{r}2.377 E+04 \\
-5 P\end{array}$ & 4.9 \\
\hline$-5.5 B 1 E+01$ & 73.0 \\
\hline $6.017 E+03$ & 21.9 \\
\hline $1.390 E+04$ & 12.4 \\
\hline $1.231 E+03$ & 14.3 \\
\hline $2.240 E+01$ & 12.4 \\
\hline $2.651 \mathrm{E}+\mathrm{O} 2$ & 3.6 \\
\hline$-1.819 E+O 2$ & 100.8 \\
\hline$-2.22 \mathrm{OE}+\mathrm{O} 2$ & 100.3 \\
\hline 1. $358 \mathrm{E}-01$ & 29.8 \\
\hline$-1.445 E+01$ & 93.1 \\
\hline $2.727 E+O 2$ & 13.9 \\
\hline $3.568 E+\infty 0$ & 9.6 \\
\hline $2.718 E+O O$ & 2.8 \\
\hline$-1.480 E+01$ & 79.3 \\
\hline $4.284 E+O O$ & 11.6 \\
\hline $5.961 E+00$ & 10.4 \\
\hline$-8 \cdot 154 E+O O$ & 89.6 \\
\hline $1.008 E+00$ & 11.2 \\
\hline $1.218 \mathrm{E}+01$ & 4.7 \\
\hline 2. $190 E+O 0$ & 5.3 \\
\hline $3.044 E+00$ & 6.5 \\
\hline $5.017 E-01$ & 7.2 \\
\hline $1.534 E+00$ & 31.8 \\
\hline$-1.028 E-02$ & 100.2 \\
\hline $5.202 E+00$ & 3.9 \\
\hline $9.906 \mathrm{E}+00$ & 11.6 \\
\hline $5.925 E+03$ & 4.6 \\
\hline $2.311 E+O O$ & 7.6 \\
\hline$-9.834 E+00$ & 81.8 \\
\hline $1.921 E+\infty O$ & 41.3 \\
\hline $1.797 \mathrm{E}+0 \mathrm{I}$ & 20.1 \\
\hline$-1.831 E+02$ & 71.5 \\
\hline$-2.064 E+00$ & 65.2 \\
\hline $9.578 E-01$ & 26.3 \\
\hline 1. $448 E+O O$ & 18.5 \\
\hline $2.731 E+02$ & 16.6 \\
\hline $2.647 E+01$ & 4.9 \\
\hline 1. $628 E+01$ & 72.8 \\
\hline $4.44 O E-01$ & 14.8 \\
\hline $5.810 E-01$ & 26.6 \\
\hline 3. $258 E+O O$ & 6.8 \\
\hline $5.435 E-01$ & 6.8 \\
\hline $2.27 O E+O O$ & 6.3 \\
\hline 8. $122 E-01$ & 34.8 \\
\hline B. $190 E-O$ & \\
\hline $7.710 \mathrm{E}+\mathrm{OO}$ & \\
\hline
\end{tabular}

\begin{tabular}{|c|c|}
\hline 2. $108 \mathrm{E}+03$ & 3.9 \\
\hline $1.870 \mathrm{E}+\mathrm{O} 3$ & 27.4 \\
\hline 4. $168 E+04$ & 4.2 \\
\hline $5.145 E+01$ & 76.1 \\
\hline 3. $409 E+03$ & 97.1 \\
\hline 1. $537 E+04$ & 12.3 \\
\hline $1.473 E+03$ & 16.0 \\
\hline 3. $449 E+01$ & 10.3 \\
\hline $4.025 E+02$ & 3.5 \\
\hline 1. $993 E+02$ & 100.8 \\
\hline $2.655 E+O 2$ & 100.3 \\
\hline 1. $083 E-01$ & 30.1 \\
\hline 1. $742 E+O 1$ & 93.2 \\
\hline 1. $203 E+O 2$ & 100.1 \\
\hline 4. $105 E+00$ & 10.3 \\
\hline $4.055 E+00$ & 2.8 \\
\hline 1. $304 E+O 1$ & 79.9 \\
\hline $7.564 E+00$ & 9.2 \\
\hline 5. $154 \mathrm{E}+00$ & 12.2 \\
\hline 6. $167 E+\infty O$ & 90.6 \\
\hline $9.970 \mathrm{E}-01$ & 10.8 \\
\hline $2.510 E+01$ & 4.0 \\
\hline $4.406 E+00$ & 4.9 \\
\hline $3.568 E+00$ & 5. 1 \\
\hline 5.795E-01 & 6.5 \\
\hline $3.993 E+00$ & 16.2 \\
\hline 8. $983 E-03$ & 100.2 \\
\hline $5.873 E+00$ & 3.9 \\
\hline 1. $391 E+01$ & 8.1 \\
\hline 1. $156 E+04$ & 4.0 \\
\hline $2.731 \mathrm{E}+00$ & 6.4 \\
\hline 5. $532 E+01$ & 14.5 \\
\hline $4.593 E+00$ & 22.5 \\
\hline $3.463 E+01$ & 12.8 \\
\hline $1.666 E+02$ & 71.6 \\
\hline $1.888 E+00$ & 65.3 \\
\hline 1. $182 E+00$ & 26.5 \\
\hline $4.123 E+0 O$ & 7.0 \\
\hline $9.981 E+01$ & 24.6 \\
\hline $5.706 E+01$ & 3.9 \\
\hline $1.014 E+01$ & 77.2 \\
\hline $5.584 E-01$ & 12.1 \\
\hline $6.425 E-01$ & 14.9 \\
\hline $4.047 E+00$ & 5.0 \\
\hline $6.176 E-01$ & 5.4 \\
\hline $3.409 E+00$ & 4.7 \\
\hline $1.026 E+00$ & 12.2 \\
\hline $5.953 E-01$ & 100.8 \\
\hline & \\
\hline
\end{tabular}


FILE ID

DUPLICATE

CLUSTER

TYPE
6101570

10
6101580

114
097

08

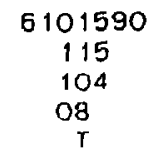

115
104

O8

6101600

116

05

ELEMENT

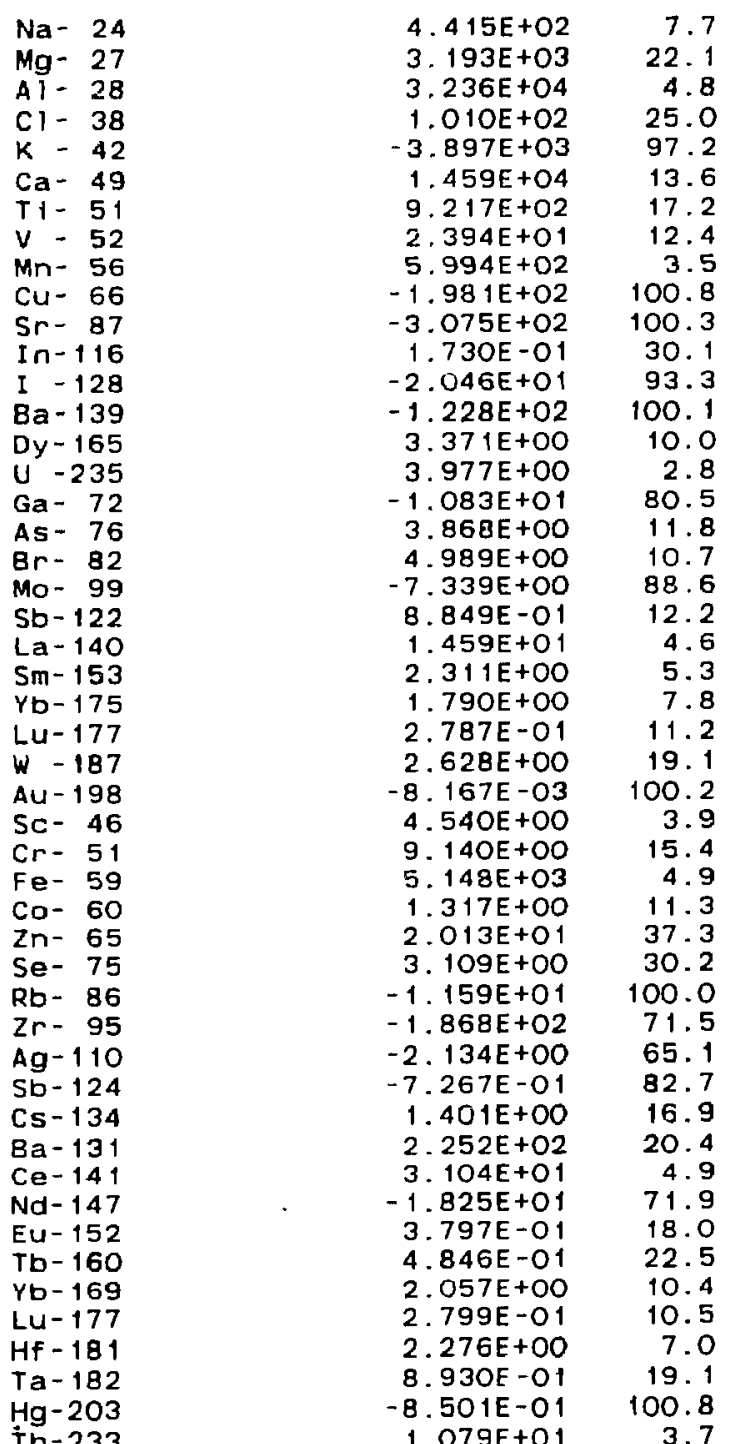

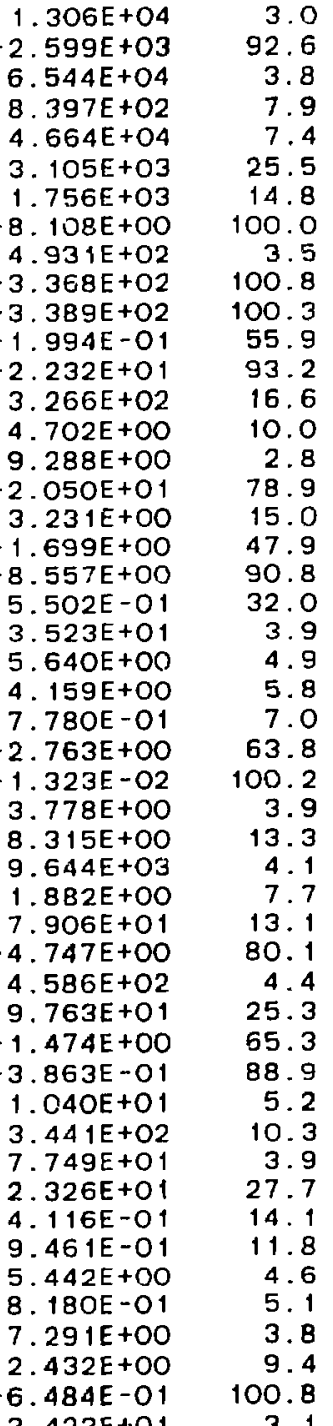
$6.07 \mathrm{OE}+03$

1. $396 E+05$

8. $192 E+O$

$7.783 E+03$

$1.076 \mathrm{E}+04$

1. $266 \mathrm{E}+03$

$-7.957 \mathrm{E}+\mathrm{OO}$

$5.541 E+O 2$

2. $786 \mathrm{E}+\mathrm{O} 2$

$-3.014 E+02$

$-1.757 \mathrm{E}-01$

$-1.989 E+O$

3. $896 \mathrm{E}+\mathrm{O} 2$

4. $532 \mathrm{E}+\mathrm{OO}$

1. $258 \mathrm{E}+\mathrm{O}$

3. $005 E+O$

$6.670 E+00$

1. $835 \mathrm{E}+\mathrm{OO}$

1. $129 E+01$

2. $092 \mathrm{E}+0 \mathrm{O}$

4. $700 E+O$

5. $840 E+00$

1. $735 E+0 O$

$-3.308 E+00$

-1 . $344 E-O 2$

4. 175E +OO

$-2.179 E+O O$

6. 13OE+O3

9. $605 \mathrm{E}-01$

1. $421 E+02$

2. $886 E+00$

3. $408 \mathrm{E}+01$

1. $513 \mathrm{E}+\mathrm{OO}$

1. $822 \mathrm{E}+\mathrm{OO}$

$2.088 \mathrm{E}+\mathrm{OO}$

4. $731 \mathrm{E}+02$

1. $099 \mathrm{E}+\mathrm{O}$

3. $401 E+01$

5. $144 \mathrm{E}-\mathrm{O}$

2. $603 E+O O$

2. $603 E+00$

3. $120 E-O$

$7.75 O E+O O$

$5.014 \mathrm{E}+00$

6. $430 E-01$

3.1
12.7
3.3
73.3
25.7
13.7
15.5
00.0
3.5
100.8
00.3
55.6
93.2
12.3
8.8
2.8
25.2
11.2
47.7
89.2
8.1
3.8
5.8
13.7
25.3
54.8
100.2
3.9
100.1
4.3
10.1
10.0
95.2
11.8
19.5
65.3
18.4
9.5
8.7
3.7
15.0
10.7
13.2
7.8
8.1
3.6
6.3
100.8
3.0

1. $523 E+03$

1. $686 \mathrm{E}+03$

2. 3O9E+O4

1. $886 E+02$

$-3.870 E+03$

$1.676 \mathrm{E}+04$

$.497 E+03$

$126 E+O 1$

$5.572 E+02$

2. $222 E+O 2$

-3. 236E+O2

1. $957 \mathrm{E}-\mathrm{O} 1$

2. $131 E+01$

4. $713 E+00$

2. $977 \mathrm{E}+0 \mathrm{O}$

$-1.600 E+01$

6. $260 E+O 0$

3. $925 \mathrm{E}+00$

$6.006 \mathrm{E}+00$

. 352E-O1

2. $665 \mathrm{E}+01$

4. $951 \mathrm{E}+0 \mathrm{OO}$

4. $823 E+O O$
$935 E-O 1$

. $233 E+00$

B. 224E-03

6. $160 E+00$

1. $212 E+01$

. $326 E+O 4$

3. $695 E+O O$

$8.677 E+O O$

$326 E+01$

$2.326 \mathrm{E}+O 2$

$592 E+O D$

8. $410 E-01$

2. $132 E+O O$

-2. $124 E+O 2$

$6.663 E+O 1$

8. $632 E-O 1$

$.717 E-01$

4. $717 E+00$

3. $294 \mathrm{E}+00$

7. 394E-O1

1. $007 E+00$

7. $930 E+O O$
4.5

5.4

15.0

97.3

11.5

18.6

11.9

100.8

100.3

30.9

93.3

100.1

9.9

80.2

80.2

13.0

13.0

11.1

11.1

4.6

5.3

5.8

25.4

100.2

3.9

4.2

6.9

83.4

63.2

29.0

65.1

65.1

78.7

11.9

66.8

4.2
34.5

12.1

16.3

6.3

6.0
27.5

100.8

4.1 


$\begin{array}{lc}\text { FILE ID } & 6101610 \\ \text { SAMPLE } & 117 \\ \text { DUPLICATE } & \text { OB9 } \\ \text { CLUSTER } & 10 \\ \text { TYPE } & L\end{array}$

6101620

118
067

01

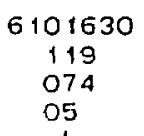

05
6 to 1640

120
077

10

ELEMENT

$\mathrm{Na}-24$

Mg- 27

AI- 28

Cl - 38

$\mathrm{Ca}-49$

$\mathrm{Ca}-49$
$\mathrm{~J} J-51$

$v-52$

$\mathrm{Mn}-56$

$\mathrm{Cu}-66$

Sr- 87

In- 116

I -128

Ba- 139

Dy-165

$u-235$

$\mathrm{Ga}-72$

$\mathrm{As}-76$
$\mathrm{Br}-82$

Mo- 99

Sb- 122

$\mathrm{La}-140$
$\mathrm{Sm}-153$

$Y \mathrm{~b}-175$

LU- 177

$w-187$

Sc -46

$\mathrm{Cr}-51$

co- 60

$\mathrm{Zn}-65$

$\mathrm{Se}-75$

Ro- 86

$\mathrm{Zr}-95$

$\mathrm{Ag}-110$

$5 b-124$

$\mathrm{Cs}-134$

Ba- 131

$\mathrm{Ce}-141$

Nd-147

Eu-152

Tb- 160

Yb- 169

Lu- 177

HF -181

Ta-182

$\mathrm{Hg}-203$

\begin{tabular}{|c|c|}
\hline $2.936 E+03$ & 3.6 \\
\hline$-1.550 E+03$ & 93.4 \\
\hline 1. $459 E+04$ & 6.3 \\
\hline$-6.233 E+01$ & 73.6 \\
\hline$-3.132 E+03$ & 97.3 \\
\hline $1.720 E+O 4$ & 11.1 \\
\hline $7.939 E+O 2$ & 22.2 \\
\hline $1.658 \mathrm{E}+01$ & 16.0 \\
\hline 4. $248 E+02$ & 3.5 \\
\hline$-2.030 E+02$ & 100.8 \\
\hline 3. $246 E+02$ & 27.1 \\
\hline $1.315 E-01$ & 23.6 \\
\hline$-1.740 E+01$ & 93.2 \\
\hline 3. $384 E+02$ & 13.2 \\
\hline 4. $84 O E+0 O$ & 8.1 \\
\hline $2.536 E+\infty O$ & 2.8 \\
\hline$-1.754 E+01$ & 79.4 \\
\hline $3.223 E+00$ & 13.3 \\
\hline 5.8 & 10.6 \\
\hline-5.5 & 90.6 \\
\hline $\begin{array}{l}1.3 \\
8.2\end{array}$ & $\begin{array}{r}8.8 \\
5.2\end{array}$ \\
\hline $2.622 \mathrm{E}+\mathrm{OO}$ & 5.1 \\
\hline 4. $.0635+00$ & 4.9 \\
\hline $7.377 E-01$ & 5.2 \\
\hline$-1.646 E+00$ & 58.6 \\
\hline$-8.354 E-03$ & 100.2 \\
\hline 4.73 & 3.9 \\
\hline 9.5321 & 11.0 \\
\hline 2. $6 B O E+O 3$ & 5.5 \\
\hline 1.16 & 10.2 \\
\hline 4.439 & 17.1 \\
\hline 2.880 & 24.8 \\
\hline 1.937 & 23.1 \\
\hline-1.312 & 71.6 \\
\hline$-1.512 E+0 O$ & 64.9 \\
\hline $1.478 E+0 O$ & 18.5 \\
\hline$-7.427 E-02$ & 100.1 \\
\hline $2.563 E$ & 14.6 \\
\hline $1.895 E+01$ & 4.9 \\
\hline$-2.974 \varepsilon+0 ?$ & 76.2 \\
\hline $4.452 \mathrm{E}-\mathrm{O}$ & 12.8 \\
\hline 5. $182 E-01$ & 16.5 \\
\hline $4.515 E+00$ & 4.4 \\
\hline $7.074 E-01$ & 5.4 \\
\hline $2.729 E+00$ & 5.5 \\
\hline$-3.363 E-01$ & 41.4 \\
\hline$-5.621 E-01$ & 100.8 \\
\hline $6.041 E+00$ & \\
\hline
\end{tabular}

\begin{tabular}{|c|c|}
\hline $5.042 E+03$ & 3.3 \\
\hline$-1.643 E+03$ & 93.1 \\
\hline 3. $290 E+04$ & 4.4 \\
\hline $1.421 \mathrm{E}+02$ & 19.7 \\
\hline$-2.987 E+03$ & 97.1 \\
\hline $9.478 E+03$ & 14.9 \\
\hline $1.114 E+03$ & 18.1 \\
\hline $2.046 E+01$ & 12.6 \\
\hline $2.332 E+O 2$ & 3.6 \\
\hline$-2.117 E+02$ & 100.8 \\
\hline$-2.252 E+02$ & 100.3 \\
\hline $1.637 \mathrm{E}-01$ & 24.6 \\
\hline$-1.471 E+01$ & 93.2 \\
\hline $4.529 E+O 2$ & 10.3 \\
\hline $3.932 E+00$ & 10.1 \\
\hline $4.632 E+O 0$ & 2.8 \\
\hline$-2,494 \mathrm{E}+01$ & 79.1 \\
\hline $6.038 E+00$ & 11.0 \\
\hline$-1.707 E+00$ & 100.3 \\
\hline$-9.705 E+00$ & 89.5 \\
\hline $1.626 E+\infty 0$ & 9.5 \\
\hline 2. $180 E+01$ & 4.3 \\
\hline 3. $253 E+00$ & 5.5 \\
\hline $2.978 E+O O$ & 6.5 \\
\hline $5.150 E-01$ & 8.3 \\
\hline $1.216 E+01$ & 12.2 \\
\hline$-1.200 E-02$ & 100.2 \\
\hline $4.897 E+O O$ & 3.9 \\
\hline 9. $197 E+00$ & 10.7 \\
\hline $7.917 E+03$ & 4.2 \\
\hline $1.739 E+00$ & 7.9 \\
\hline $9.252 E+O f$ & 11.6 \\
\hline $2,705 E+00$ & 32.0 \\
\hline $2.503 E+01$ & 16.7 \\
\hline$-1.493 E+02$ & 71.5 \\
\hline$-1.715 E+O O$ & 65.0 \\
\hline 1. $168 E+00$ & 27.9 \\
\hline 1. $197 E+00$ & 15.2 \\
\hline $5.377 \mathrm{E}+02$ & 8.9 \\
\hline $4.961 E+01$ & 3.9 \\
\hline$-9.753 E+00$ & 76.6 \\
\hline $4.479 \mathrm{E}-01$ & 13.2 \\
\hline $8.156 \mathrm{E}-01$ & 15.2 \\
\hline 4. $196 E+00$ & 4.6 \\
\hline $6.333 E-01$ & 5.3 \\
\hline $4.339 E+00$ & 4.4 \\
\hline $8.068 E-01$ & 16.2 \\
\hline$-6.468 E-01$ & 100.8 \\
\hline $1.294 E+01$ & 3.3 \\
\hline
\end{tabular}

$6.321 E+02$ 3. $680 E+03$

$3.470 E+04$

6. $220 \mathrm{OE}+\mathrm{O} 1$

-3. $154 \mathrm{E}+\mathrm{O} 3$

1. $371 E+04$

1. $881 \mathrm{E}+03$

4. $336 \mathrm{E}+01$

4. $711 \mathrm{E}+02$

$-1.774 E+O 2$

$-2.562 E+O 2$

$-1.239 \mathrm{E}-01$

$-1.699 E+0$

- 1. $148 \mathrm{E}+\mathrm{O} 2$

2. $771 \mathrm{E}+\mathrm{OO}$

$2.626 E+00$

1. $35 \mathrm{OE}+\mathrm{O} 1$

4. $74 \mathrm{OE}+\mathrm{OO}$

$3.656 E+O O$

$4.739 \mathrm{E}+\mathrm{OO}$

$5.698 E-O$

$1.834 E+O$

2. $830 E+O 0$

2. $145 \mathrm{E}+00$

4. $041 \mathrm{E}-01$

3. $055 \mathrm{E}+\mathrm{OO}$

$-6.707 E-03$

$5.427 \mathrm{E}+00$

9. $687 \mathrm{E}+03$

2. $391 E+O 0$

5. $380 E+01$

3. $816 \mathrm{E}+\mathrm{OO}$

2. $005 \mathrm{E}+\mathrm{O}$

2. $191 \mathrm{E}+02$

2.191E+O2

1. $510 E+O 0$

2.

2. $964 \mathrm{E}+00$

1. $347 E+02$

3. $563 E+01$

$-8.082 \mathrm{E}+00$

4. 317E-O 1

4.512E-O

2. $747 E+00$

3. $964 E-01$

2. $405 E+00$

5. 949E-01

$-5.586 E-O$

9. $118 E+O 0$
5.6

14.2

32.1

97.2

11.6
12.7

8.2

3.5

100.8

00.3

56.1

100.1

13.0

2.8

80.2

12.4

90.4

14.7

4.2
4.9

6.1

6.7

24.7

3.9

7.4

4.1

14.2

30.5

16.2

23.7

65.2

27.4

8. 0

4.5

77.4

12.8

18.1

6.3
6.9

5.5

17.1

00.8 


$\begin{array}{lc}\text { FILE ID } & 6101650 \\ \text { SAMPLE } & 121 \\ \text { DUPLICATE } & 086 \\ \text { CLUSTER } & 10 \\ \text { TYPE } & L\end{array}$




$\begin{array}{lc}\text { FILE ID } & 6101690 \\ \text { SAMPLE } & 125 \\ \text { DUPLICATE } & 105 \\ \text { CLUSTER } & \text { O8 } \\ \text { TYPE } & T\end{array}$

$$
\begin{gathered}
6101700 \\
126 \\
100 \\
08 \\
T
\end{gathered}
$$

6101720

128

090
07

\section{ELEMENT}

$\mathrm{Na}-24$
$\mathrm{Mg}-27$

Al- 28

Cl- 38

$K-42$

$\mathrm{Ca}-49$

$v=52$

Mn- 56

Cu- 66

$\mathrm{Sr}^{-} 87$

In -116
$I-128$

Ba-139

Dy -165

U -235

$\mathrm{Ga}-72$

$\mathrm{AS}-76$
$\mathrm{BC}-\mathrm{8}$

$\mathrm{Br}-82$

So-122

La- 140

$\mathrm{Sm}-153$

$\mathrm{Yu}-175$
$\mathrm{Lu}-177$

W-187

$A U-198$

Sc- 46

Cr -51

$\mathrm{Fe}-59$

Co- 60

$\mathrm{Se}-75$

RD- 86

$2 \Gamma-95$

$A g-110$

Sb-124

C5- 134

Ba-131

$\mathrm{Ce}-141$
$\mathrm{Nd}-147$

Eu- 152

Tb-160

Yb- 169

$-u-177$

Hf - 181

Ta-1B2

\begin{tabular}{|c|c|}
\hline 7. $319 E+03$ & 3.1 \\
\hline $6.136 E+03$ & 14.3 \\
\hline $1.025 E+05$ & 3.4 \\
\hline $1.500 E+02$ & 17.9 \\
\hline $1.906 \mathrm{E}+04$ & 11.8 \\
\hline $1.043 E+04$ & 13.4 \\
\hline $1.314 E+03$ & 18.7 \\
\hline$-7.286 E+O O$ & 100.0 \\
\hline 4. $277 E+02$ & 3.5 \\
\hline$-2.635 \mathrm{E}+\mathrm{O} 2$ & 100.8 \\
\hline$-2.838 E+02$ & 100.3 \\
\hline $1.754 E-01$ & 22.6 \\
\hline$-1.857 E+01$ & 93.2 \\
\hline $3.395 E+02$ & 12.0 \\
\hline $6.34 O E+O O$ & B. 1 \\
\hline 1. $256 \mathrm{E}+\mathrm{O}_{1}$ & 2.8 \\
\hline $1.913 E+01$ & 17.8 \\
\hline $6.865 E+00$ & 9.2 \\
\hline$-1.513 E+00$ & 47.8 \\
\hline 5.0 & 10.2 \\
\hline 1. $5 \nmid 6 E+O O$ & 9.6 \\
\hline 4. $534 \mathrm{E}+01$ & 3.7 \\
\hline 7. $223 \mathrm{E}+00$ & 5.1 \\
\hline $4.223 E+00$ & 5.4 \\
\hline 7. $102 E-01$ & 8.4 \\
\hline $2.427 E+\infty 0$ & 18.1 \\
\hline$-1.250 E-02$ & 100.2 \\
\hline $4.618 \mathrm{E}+00$ & 3.9 \\
\hline $6.386 E+00$ & 18.9 \\
\hline $8.028 E+03$ & 4.2 \\
\hline 1. $245 E+00$ & 8.9 \\
\hline $1.078 E+02$ & 11.3 \\
\hline$-1.3025+00$ & 100.0 \\
\hline $8.588 E+01$ & 7.2 \\
\hline 1. $164 \mathrm{E}+02$ & 18.6 \\
\hline$-1.471 E+\infty O$ & 65.0 \\
\hline $1.472 E+\infty O$ & 17.9 \\
\hline 4. $408 E+00$ & 6.8 \\
\hline $4.019 E+02$ & 8.6 \\
\hline $9.675 \mathrm{E}+01$ & 3.8 \\
\hline $3.415 \mathrm{E}+\mathrm{O} 1$ & 20.0 \\
\hline & \\
\hline 1. $105 E+\infty 0$ & 10.2 \\
\hline $4.490 E+O O$ & 5.7 \\
\hline $6.796 \mathrm{E}-01$ & 5.3 \\
\hline B. $364 E+00$ & 3.6 \\
\hline 3. $586 \mathrm{E}+00$ & 7.4 \\
\hline$-6.217 E-01$ & 100.8 \\
\hline & \\
\hline
\end{tabular}

$\mathrm{Hg}-203$

Th-233

5. $428 \mathrm{E}+03$

3. $158 \mathrm{E}+03$

$6.056 \mathrm{E}+04$

5. $369 E+01$

7. $605 E+03$

1. $136 E+04$

2. $750 E+O 3$

3. $761 E+O$

2. $830 \mathrm{E}+\mathrm{O} 2$

1.739E +

1. $849 \mathrm{E}-01$

1. $212 E+O 1$

4. $846 \mathrm{E}+\mathrm{O} 2$

3. $986 \mathrm{E}+00$

6. $151 \mathrm{E}+\mathrm{OO}$

1. $344 E+0$

3. $729 \mathrm{E}+00$

. $658 \mathrm{E}+0 \mathrm{O}$

. $027 E+00$

2. $596 \mathrm{E}+\mathrm{O} 1$

3. $164 \mathrm{E}+\mathrm{OO}$

2. $217 \mathrm{E}+\mathrm{OO}$

3. $291 \mathrm{E}-01$

2. $182 \mathrm{E}+0 \mathrm{O}$

1. $218 \mathrm{E}-\mathrm{O} 2$

$5.832 E+00$

1. $384 \mathrm{E}+01$

B. $858 \mathrm{E}+03$

1. $927 \mathrm{E}+\mathrm{OO}$

6. $139 \mathrm{E}+\mathrm{O} 1$

1. $908 \mathrm{E}+00$

3. $.929 E+0$

1. $458 \mathrm{E}+\mathrm{O} 2$

1. $619 \mathrm{E}+00$

3. $904 \mathrm{E}+00$

6. $256 \mathrm{E}+\mathrm{O} 2$

5. $275 \mathrm{E}+01$

9. $169 \mathrm{E}+00$

5. 193E-O 1

$5.37 \mathrm{OE}-\mathrm{O}$

2. $852 \mathrm{E}+00$

4. $36 \mathrm{E} \equiv-01$

4. $119 E+O O$

1. $629 E+O O$

$-6.133 E-01$

2. 117E+O1

$\begin{array}{r}3.2 \\ 21.0 \\ 3.6 \\ 72.7 \\ 15.4 \\ 12.4 \\ 8.1 \\ 9.1 \\ 3.5 \\ 00.8 \\ 25.2 \\ 22.6 \\ 93.2 \\ 8.3 \\ 8.0 \\ 2.8 \\ 78.8 \\ 11.7 \\ 13.5 \\ 42.5 \\ 13.0 \\ 3.9 \\ 5.7 \\ 8.2 \\ 14.5 \\ 54.6 \\ 00.2 \\ 3.9 \\ 7.7 \\ 4.0 \\ 6.7 \\ 12.8 \\ 83.1 \\ 10.4 \\ 73.3 \\ 76.9 \\ 19.8 \\ 6.7 \\ 7.7 \\ 3.9 \\ 76.4 \\ 10.3 \\ 16.3 \\ 6.0 \\ 6.1 \\ 4.2 \\ 9.9 \\ 00.8 \\ 3.1 \\ 39 \\ \hline\end{array}$

2. $348 \mathrm{E}+03$

2. $31 \mathrm{BE}+\mathrm{O} 3$

3. $946 \mathrm{E}+04$

$-4.993 E+01$

$-4.993 E+01$
$-3.184 E+03$

$380 \mathrm{E}+04$

. $595 \mathrm{E}+0$

. $595 E+03$

$4.536 \mathrm{E}+\mathrm{O}$

1. $878 \mathrm{E}+0$

1.878E+O2

2. $467 E+O 2$

$-1.619 E+01$

1. $547 \mathrm{E}+02$

$250 E+00$

2. $708 \mathrm{E}+00$

$-7.438 \mathrm{E}+\mathrm{OO}$

3. $644 \mathrm{E}+\mathrm{OO}$

3. $127 \mathrm{E}+\mathrm{OO}$

$5.027 \mathrm{E}+00$

7. $291 E-O 1$

$.284 E+O 1$

$.746 \mathrm{E}+00$

1. $541 E+00$

2. $924 \mathrm{E}-01$

2. $240 E+00$

$6.658 \mathrm{E}-\mathrm{O}$

$5.920 E+O O$

2. $720 E+O$

$7.262 \mathrm{E}+\mathrm{OJ}$

2. $090 \mathrm{O}+\mathrm{OO}$

2. $497 \mathrm{E}+\mathrm{O}$

$2.597 E+00$

3. $160 E+01$

1. $794 E+02$

$-2.068 E+O O$

6. $450 E-01$

3. $583 \mathrm{E}+\mathrm{OO}$

1. $753 E+02$

2. $312 E+01$

1. $587 \mathrm{E}+01$

3. $386 \mathrm{E}-\mathrm{O} 1$

4. $003 \mathrm{E}-01$

1. $7 \mathrm{~B} 5 \mathrm{E}+0 \mathrm{O}$

$2.851 \mathrm{E}-\mathrm{O}$

2. $667 \mathrm{E}+\mathrm{OO}$

1. $120 E+00$

$-7.999 E-01$

8. $881 E+O O$
3.7

4.1

75.4

8.8

8.3

5

100.8

00. 3

25.9

93.2

19.5

10.7

2.8
79.3

11.0

12.4

23.0

11.9

4.2

7.9

10. 6

17.3

00.2

4.4

7.8

39.6

15.2

71.6

65.1

80.1

8. 4

5. 7

73.3

25.1

10.4

10.0

13.6

100.8

3.7 


$\begin{array}{lc}\text { FILE ID } & 6101730 \\ \text { SAMPLE } & 129 \\ \text { DUPLICATE } & 099 \\ \text { CLUSTER } & 10 \\ \text { TYPE } & \text { L }\end{array}$

6101740

130
065

1
6101750

131
064

10
6101760

132
061

07

ELEMENT

$\mathrm{Na}-24$

$\mathrm{Mg}-27$

A1 - 28

Cl -38

K -42

Ca- 49

$v-5$

$\mathrm{Mn}-56$

Cu- 66

$\mathrm{Sr}-87$

In- 116

I -128

$B a-139$
$D y-165$

$D y-165$
$U-235$

Ga- 72

A5 - 76

$\mathrm{Br}-82$

Mo- 99

Sb-122

La- 140

Sm- 153

Yo- 175

LU- 177

$W-187$
Au

$\mathrm{Au}-198$

$\mathrm{SC}-46$

Fe- 59

Co- 60

$2 n-65$

$\mathrm{Se}-75$

Rb- 86

$\mathrm{Zr}-95$

Ag- 110

Sb-124

$\mathrm{Cs}-134$

$8 a-131$

$\mathrm{Ce}-141$
$\mathrm{Nd}-147$

Eu-152

D - 160

bo- 169

Vb- 169

Lu- 177

Ta- 182

\& $\begin{array}{r}\mathrm{Hg}-203 \\ \mathrm{Th}-233\end{array}$

$\begin{array}{rr} & \\ 1.094 E+O 3 & 4.8 \\ 2.549 E+O 3 & 21.8 \\ 3.918 E+O 4 & 4.3 \\ 9.72 O E+O 1 & 23.1 \\ -2.996 E+O 3 & 97.1 \\ 1.472 E+O 4 & 12.9 \\ 1.362 E+O 3 & 13.4 \\ 2.152 E+O 1 & 13.5 \\ 4.321 E+O 2 & 3.5 \\ -1.649 E+O 2 & 100.8 \\ -2.305 E+O 2 & 100.3 \\ 1.316 E-O 1 & 28.6 \\ -1.512 E+O 1 & 93.3 \\ -9.072 E+O 1 & 100.1 \\ 4.395 E+O O & 8.2 \\ 4.700 E+O O & 2.8 \\ -9.9 n 1 E+C O & 79.6 \\ 7.544 E+O O & 9.2 \\ 5.353 E+O O & 10.5 \\ -7.929 E+O O & 89.1 \\ 9.614 E-O 1 & 12.2 \\ 2.201 E+O 1 & 4.1 \\ 3.412 E+O O & 5.2 \\ 2.710 E+O O & 6.6 \\ 4.524 E-O 1 & 9.2 \\ 1.990 E+O O & 20.4 \\ -9.378 E-O 3 & 100.2 \\ 4.782 E+O O & 3.9 \\ 8.868 E+O O & 9.7 \\ 5.024 E+O 3 & 4.6 \\ 1.402 E+O O & 8.4 \\ 4.410 E+O 1 & 17.7 \\ 2.469 E+O O & 28.3 \\ 1.604 E+O 1 & 21.9 \\ -1.45 O E+O 2 & 71.7 \\ -1.72 B E+O O & 65.1 \\ 9.457 E-O 1 & 31.4 \\ 1.578 E+O O & 13.0 \\ 1.237 E+O 2 & 18.2 \\ 4.816 E+O 1 & 4.0 \\ -9.527 E+O O & 76.1 \\ 4.284 E-O 1 & 14.2 \\ 6.051 E-O 1 & 15.9 \\ 3.512 E+O O & 5.2 \\ 4.642 E-01 & 5.9 \\ 3.260 E+O O & 4.8 \\ 1.094 E+O O & 12.5 \\ -1.295 E-01 & 100.8 \\ 1.432 E+O 1 & 3.3 \\ & \end{array}$

\begin{tabular}{|c|c|}
\hline $4.529 E+03$ & \\
\hline$-1.482 E+03$ & 92 \\
\hline $2.562 E+O 4$ & \\
\hline$-6.315 E+01$ & \\
\hline$-2.797 E+03$ & \\
\hline $1.055 E+04$ & 14 \\
\hline 1. $401 E+03$ & 12 \\
\hline $2.633 E+01$ & 11 \\
\hline 2. $406 E+02$ & \\
\hline$-1.964 E+02$ & 100 \\
\hline$-2.12 O E+O 2$ & 100 \\
\hline$-1.183 E-01$ & 56 \\
\hline$-1.394 E+01$ & 93 \\
\hline 5. $328 E+O 2$ & \\
\hline $3.834 E+O 0$ & \\
\hline 3. OOOE +OO & \\
\hline$-1.062 E+01$ & 78 \\
\hline $8.999 r+00$ & \\
\hline $2.895 E+O O$ & 20 \\
\hline$-5.853 E+O O$ & 90 \\
\hline $1.483 E+00$ & \\
\hline $1.510 E+01$ & \\
\hline 2. $709 E+00$ & \\
\hline 2. $558 E+00$ & \\
\hline $4.003 E-01$ & \\
\hline $5.561 E+00$ & 12 \\
\hline$-9.219 E-03$ & 100 \\
\hline $4.078 E+00$ & 3 \\
\hline 1. $190 E+01$ & 12 \\
\hline $1.079 E+04$ & \\
\hline 1. $956 E+00$ & 8 \\
\hline$-1.246 E+01$ & 63 \\
\hline$-2.054 E+00$ & 101 \\
\hline $2.683 E+01$ & 17 \\
\hline$-1.811 E+02$ & 71 \\
\hline$-2.053 E+00$ & 65 \\
\hline$-6.967 E-01$ & 78 \\
\hline $2.090 E+00$ & 11. \\
\hline $5.338 \mathrm{E}+\mathrm{O} 2$ & 11. \\
\hline $3.313 E+01$ & 4. \\
\hline$-1.627 E+01$ & 72 \\
\hline $3.211 E-01$ & 17. \\
\hline 5. $109 \mathrm{E}-01$ & \\
\hline 3. $349 E+O O$ & 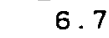 \\
\hline 4. $162 E-01$ & \\
\hline 3. $13 O E+O O$ & 5. \\
\hline $8.743 E-01$ & \\
\hline$-8.156 E-01$ & 100. \\
\hline $8.840 E+O O$ & \\
\hline
\end{tabular}

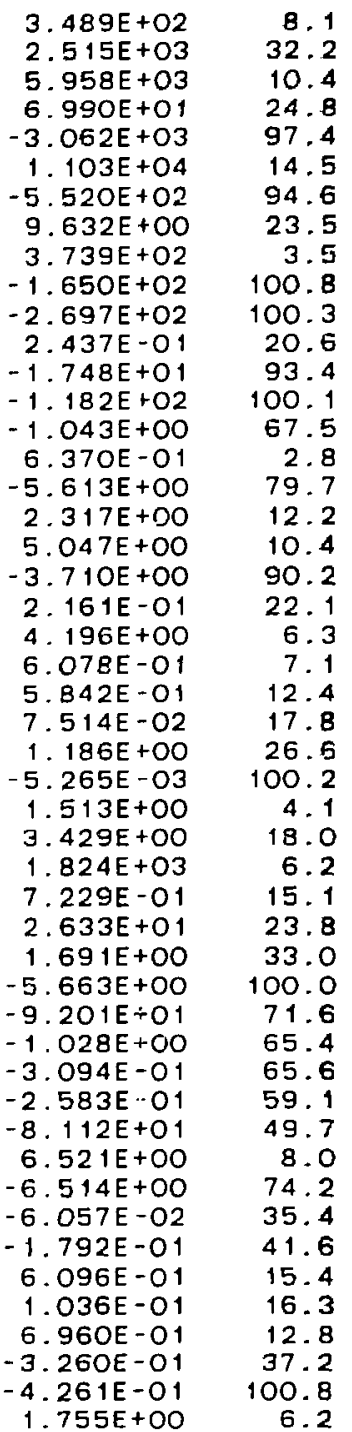

. 4

4.8

4.5

3.5

0.8

00.3

3. 4

9
67.5

2.8

2.2

90.2

6.3

17.8

00. 2

4.1

6.2

3.1

3.0

1.6

65.6

59.1

5. 4

1.6

16. 3

7.2

6.2 
8

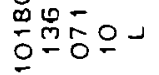
(0)

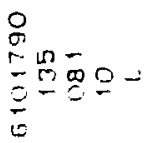

$\frac{D}{\infty}=\frac{1}{2}=\frac{0}{0}$

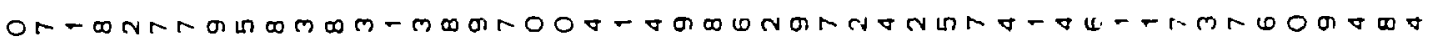
में

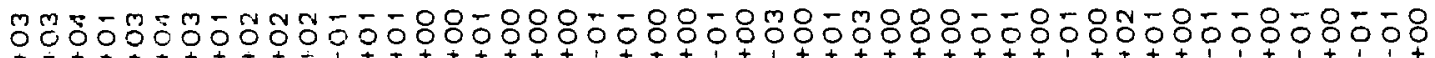

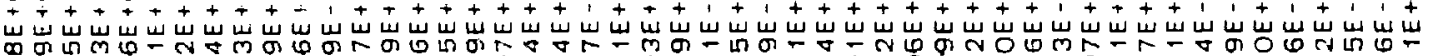

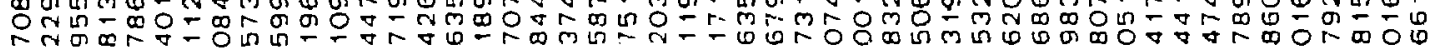

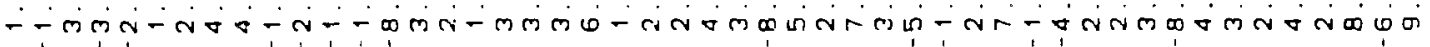

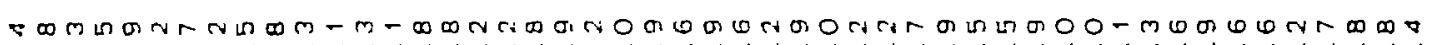
m m

3⿻ำ +

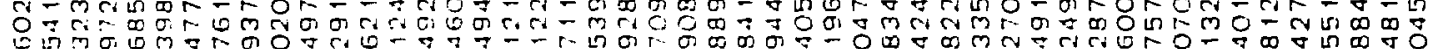

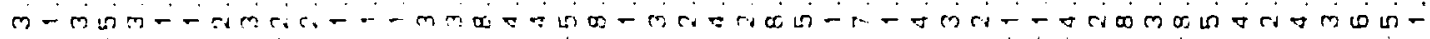

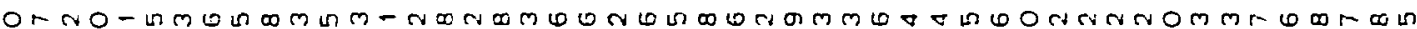

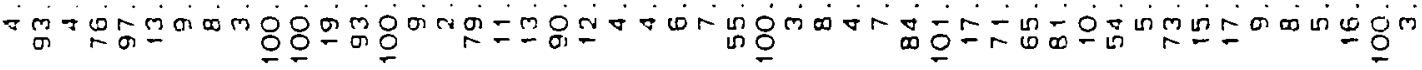

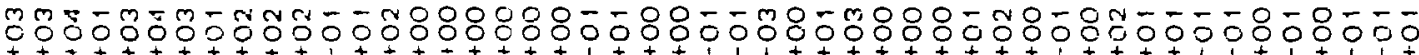
+
$\omega$ G

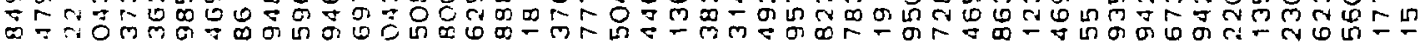
- -

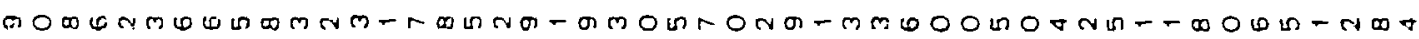
गु

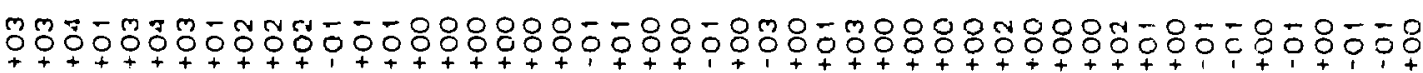

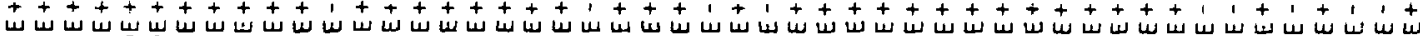

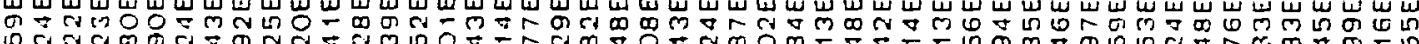

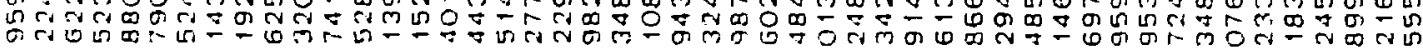

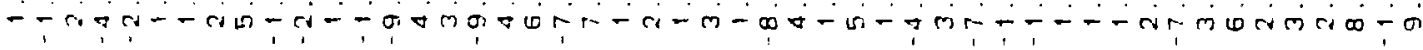

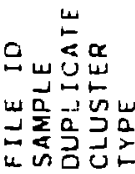

攴:

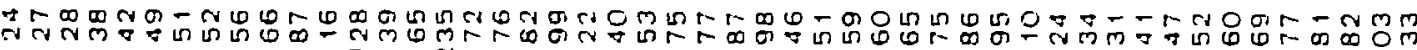

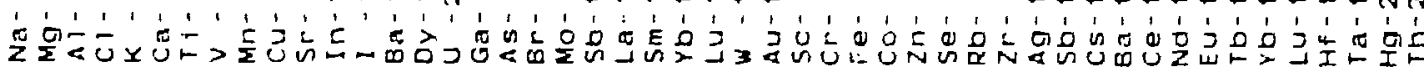




$\begin{array}{lc}\text { FILE ID } & 6101810 \\ \text { SAMPLE } & 137 \\ \text { DUPLICATE } & 102 \\ \text { CLUSTER } & 08 \\ \text { TYPE } & T\end{array}$

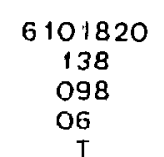

OG

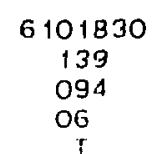

06
6101840

140

05

ELEMENT

$\mathrm{Na}-24$

$\mathrm{Mg}^{-} \quad 27$

A) - 28

C1- 38

$K-42$

Ca- 49

$T 1-51$
$v-52$

$\mathrm{Mn}-5 \mathrm{G}$

Cu- 66

Sr- 87

In- 116

$1-128$
$B a-139$

Dy -165
$U-235$

Ga- 72

As- 76

$\mathrm{Br}-82$

Sb- 122

La- 140

$\mathrm{Sm}-153$

$Y b-175$

$L U-177$
$W-187$

Au- 198

$\mathrm{SC}-46$

Cr -51

Fe- 59

$\mathrm{CO}-60$

$\mathrm{Lil}-65$
$\mathrm{Se}-75$

Rb- 86

$\mathrm{Zr}-95$

$\mathrm{Ag}^{-110}$

5b- 124

Cs -134

$\mathrm{Ba}-131$

$\mathrm{Ce}-141$

$\mathrm{Nd}-147$

Eu- 152

Yb- 169

$Y b-169$
$L u-177$

$\mathrm{LU}-177$
$\mathrm{HF}-181$

HF- 18

$\mathrm{Ta}-182$

ज $\quad \mathrm{Hg}-203$

\begin{tabular}{|c|c|}
\hline $6.617 E+03$ & 3.2 \\
\hline $5.472 E+03$ & 15.8 \\
\hline $9.088 \mathrm{E}+04$ & 3.6 \\
\hline $3.633 E+O 2$ & 10.5 \\
\hline $1.610 E+04$ & 11.8 \\
\hline $7.402 E+03$ & 18.2 \\
\hline $1.161 E+03$ & 16.4 \\
\hline B. $736 E+O O$ & 23.1 \\
\hline $5.483 E+02$ & 3.5 \\
\hline $2.786 E+O 2$ & 100.8 \\
\hline$-3.099 E+02$ & 100.3 \\
\hline$-1.798 E-01$ & 55.6 \\
\hline$-2.056 \mathrm{E}+01$ & 93.2 \\
\hline$-1.264 \mathrm{E}+02$ & 100.1 \\
\hline $5.117 E+O O$ & 8. 6 \\
\hline $1.099 E+01$ & 2.8 \\
\hline$-1.275 E+01$ & 79.0 \\
\hline $6.905 E+00$ & 9.5 \\
\hline $2.74 \mathrm{BE}+O O$ & 18.8 \\
\hline 1. $598 \varepsilon+00$ & 11.9 \\
\hline $9.750 E-$ & 13.1 \\
\hline 3. $229 E+O 1$ & 3.8 \\
\hline 5. $266 \mathrm{E}+$ & 4.9 \\
\hline $2.440 E+C$ & 7.8 \\
\hline $3.792 \mathrm{E}-01$ & 17.0 \\
\hline$-1.612 E+00$ & 51.7 \\
\hline$-1.056 \mathrm{E}-02$ & 100.2 \\
\hline 4. $932 \mathrm{E}+00$ & 3.9 \\
\hline$-3.084 E+00$ & 100.1 \\
\hline $6.378 E+0$ & 4.6 \\
\hline 6.86 & 16.5 \\
\hline $7.273 E+O$ & 13.4 \\
\hline $3.846 E+C$ & 32.0 \\
\hline $1.656 \mathrm{E}+02$ & 6.7 \\
\hline - $1.870 E+02$ & 86.7 \\
\hline$-2.137 E+C$ & 65.2 \\
\hline 1. $147 E+C$ & 25.6 \\
\hline $4.179 E+O$ & 7.6 \\
\hline 1. $289 E+02$ & 24.0 \\
\hline 7. $109 E+01$ & 4.0 \\
\hline $2.926 \mathrm{E}+01$ & 25.3 \\
\hline 4. $592 \mathrm{E}-01$ & 14.1 \\
\hline $7.171 \mathrm{E}-\mathrm{OH}$ & \\
\hline $3.432 E+00$ & 8.5 \\
\hline & \\
\hline $4.651 E+\infty$ & 4.7 \\
\hline $1.799 E+00$ & \\
\hline $8.896 \mathrm{E}-01$ & 100.8 \\
\hline $2.944 E+01$ & 3.2 \\
\hline
\end{tabular}

\begin{tabular}{|c|c|}
\hline 8. $759 E+03$ & 3.1 \\
\hline $3.614 \mathrm{E}+03$ & 24.9 \\
\hline 1. $119 E+05$ & 3.4 \\
\hline$-8.256 E+01$ & 71.9 \\
\hline$: \quad 676 E+04$ & 11.5 \\
\hline $9.450 E+03$ & 13.4 \\
\hline $3.616 E+03$ & 7.2 \\
\hline$-6.957 E+00$ & 100.0 \\
\hline $2.533 \mathrm{E}+\mathrm{O} 2$ & 3.6 \\
\hline$-2.698 E+02$ & 100.8 \\
\hline $5.455 E+O 2$ & 17.7 \\
\hline 3. $210 E-01$ & 19.9 \\
\hline$-1.676 E+01$ & 93.0 \\
\hline $1.513 E+03$ & 6.5 \\
\hline 4. $607 E+00$ & 9.2 \\
\hline $5.255 E+00$ & 2.8 \\
\hline$-1.721 E+01$ & 78.9 \\
\hline $1.943 E+00$ & 19.8 \\
\hline$-1.703 E+00$ & 47.9 \\
\hline$-8.608 E+00$ & 91.2 \\
\hline 1. $362 E+00$ & 10.5 \\
\hline $5.403 E+01$ & 3.7 \\
\hline 8. $430 E+O 0$ & 4.8 \\
\hline $2.552 E+O O$ & 7.3 \\
\hline $3.722 \mathrm{E}-01$ & 13.1 \\
\hline$-2.67 O E+O O$ & 63.0 \\
\hline$-1.389 E-02$ & 100.2 \\
\hline $5.979 E+00$ & 3.9 \\
\hline $5.884 E+O O$ & 28.0 \\
\hline $9.817 E+03$ & 4.1 \\
\hline $6.538 E-01$ & 14.4 \\
\hline 1. $221 \mathrm{E}+\mathrm{O} 2$ & 11.1 \\
\hline$-1.407 E+00$ & 94.3 \\
\hline $9.462 \mathrm{E}+01$ & 7.3 \\
\hline $3.532 \mathrm{E}+\mathrm{O} 2$ & 16.1 \\
\hline$-1.692 \mathrm{E}+00$ & 65.0 \\
\hline $1.057 E+00$ & 24.4 \\
\hline $3.482 E+00$ & 7.7 \\
\hline 1. $416 \mathrm{E}+03$ & 5.3 \\
\hline 1. $149 \mathrm{EE}+\mathrm{O} 2$ & 3.8 \\
\hline $4.332 E+01$ & 17.6 \\
\hline $1.374 \mathrm{E}+00$ & 7.3 \\
\hline $7.683 E-01$ & 15.8 \\
\hline 3. $15 O E+O O$ & 6.6 \\
\hline 3. 37OE-O1 & 8.3 \\
\hline 1. $470 E+01$ & 3.4 \\
\hline $2.706 E+\infty 0$ & B. B \\
\hline$-6.777 \mathrm{E}-01$ & 100.8 \\
\hline $2.207 E+01$ & 3.2 \\
\hline
\end{tabular}

\begin{tabular}{|c|c|}
\hline 7. $942 E+03$ & 3.1 \\
\hline 4. $503 E+03$ & 14.2 \\
\hline 1. $137 E+05$ & 3.4 \\
\hline$-7.161 E+01$ & 72.2 \\
\hline $1.560 E+04$ & 12.5 \\
\hline $1.067 E+04$ & 14.6 \\
\hline $1.073 E+03$ & 17.8 \\
\hline$-6.180 E+O O$ & 100.0 \\
\hline $2.147 \mathrm{~F}+\mathrm{O} 2$ & 3.6 \\
\hline$-2.367 E+02$ & 100.8 \\
\hline$-2.191 E+O 2$ & 100.3 \\
\hline$-1.370 E-01$ & 55.7 \\
\hline$-1.4 O O E+01$ & 93.2 \\
\hline $9.174 E+02$ & 7.3 \\
\hline $5.884 E+O O$ & 7.9 \\
\hline $1.191 \mathrm{E}+01$ & 2.8 \\
\hline$-2.034 E+01$ & 79.2 \\
\hline$-1.600 E+00$ & 86.3 \\
\hline$-2.046 E+00$ & 47.8 \\
\hline$-1.358 E+01$ & 89.5 \\
\hline $9.172 E-01$ & 16.2 \\
\hline 5. $144 E+01$ & 3.8 \\
\hline 6. $887 E+O O$ & 4.9 \\
\hline $2.361 E+O O$ & 10.4 \\
\hline $3.786 E-01$ & 21.4 \\
\hline$-3.096 \mathrm{E}+00$ & 55.6 \\
\hline$-1.697 E-02$ & 100.2 \\
\hline $3.934 E+00$ & 3.9 \\
\hline $2.556 \mathrm{E}+00$ & 100.1 \\
\hline 1. $164 E+O A$ & A. 0 \\
\hline $7.880 E-01$ & 13.2 \\
\hline $1.494 \mathrm{E}+\mathrm{O} 2$ & 10.3 \\
\hline$-3.127 E+00$ & 92.2 \\
\hline $6.055 E+01$ & 9.4 \\
\hline$-1.290 E+02$ & 97.0 \\
\hline$-1.746 E+00$ & 65.4 \\
\hline $1.053 E+00$ & 24.5 \\
\hline $2.612 \mathrm{E}+\infty 0$ & 9.1 \\
\hline $9.364 \mathrm{E}+02$ & 6.5 \\
\hline $1.217 \mathrm{E}+\mathrm{O} 2$ & 3.7 \\
\hline $3.832 E+01$ & 18.9 \\
\hline $5.498 \mathrm{E}-01$ & 11.5 \\
\hline $1.128 \mathrm{E} \div .00$ & 12.7 \\
\hline 3. $205 \mathrm{E}+00$ & 6.6 \\
\hline $4.566 \mathrm{E}-01$ & 6.8 \\
\hline $6.513 E+O O$ & 4.0 \\
\hline $3.976 E+00$ & 7.5 \\
\hline$-7.343 E-01$ & 100.8 \\
\hline 5. $183 E+01$ & 3.0 \\
\hline
\end{tabular}

1. $849 E+03$

3.864

C. 35BE

- 6 . 657E+O

3. $138 E+03$

-3. $138 E+03$

1. $234 E+04$

$2.704 \mathrm{E}+03$

$5.258 E+O$

3. $791 E+02$

1. $795 \mathrm{E}+02$

1.226E+O2

1. $971 \mathrm{E}-01$

1. $452 E+O 1$

$4.85 O E+0 O$

3. $745 \mathrm{E}+O O$

3.745E+OO

3. $324 \mathrm{E}+00$

3. $24 \mathrm{E}+0 \mathrm{O}$

2.5O8E+OO

1. $272 E+0 O$

$1.034 E+00$

2. $781 E+01$
$4.262 E+O 0$

4. $262 E+O 0$

3. $697 \mathrm{E}+00$

4. $903 E-01$

2. 117E+OO

7. $535 \mathrm{E}+00$

2. $247 E+O$

1. $343 E+O$

3. $090 E+00$

3. $174 E+O$

- $249 E+O O$

$3.542 E+O 1$

2. $065 \mathrm{E}+02$

$-2.361 E+00$

$6.448 E-01$

$5.703 E+00$

1. $755 E+C 2$

5. $916 \mathrm{E}+\mathrm{O}$

$-1.795 E+01$

$7.727 E-01$

5. 369E-O

4. $019 E+O O$

5. $366 E-01$

f. $162 \mathrm{E}$

$-9.078 E-01$

1. $472 \mathrm{E}+\mathrm{O} 1$
3. 9

16.9

75.4

75.4

12.4

7.6

3. 5

00.8

100.3

93.3

13.5

2.8

2.8
79.6

11. 8

13.4
5.6

3. 8

5.3

7.0

25.6

100. 2

7.7

4.0
6.4

15.5

66.9

14.9

65.2

81.5

6.6
27.7

4. 4

73.4

10.5

6.1

6. 9

13.5

100.8 


$\begin{array}{lc}\text { FILE ID } & 6101850 \\ \text { SAMPLE } & 141 \\ \text { OUPLICATE } & 080 \\ \text { CLUSTER } & 05 \\ \text { TYPE } & L\end{array}$

$$
\begin{gathered}
6101860 \\
142 \\
101 \\
08 \\
T
\end{gathered}
$$

$$
\begin{gathered}
6101870 \\
143 \\
093 \\
07 \\
4
\end{gathered}
$$

\section{ELEMENT}

\section{$\mathrm{Na}-24$}

Mg- 27

A) - 28

C) -38

$k-42$

$\mathrm{Ca}-49$

$T+51$
$v-52$

Mn- 56

Cu- 66

$5 r-87$

I) -116
I -128

$1-128$
$B a-139$

Dy -165

$U-235$

Ga- 72

AS- 76

$\mathrm{Br}-82$

Mo- 99

So- 122

Ym- 153

LU-177

W- 187

Au- 198

SC- 46

$\mathrm{Cr}-51$

Fe- 59

Co- 60

$2 n-65$
$5 e-75$

$\mathrm{Se}-75$
$\mathrm{Ra}-\mathrm{B} 6$

$\mathrm{Rb}-\mathrm{B6}$

Ag- 110

Sb-124

Cs-134

$8 a-131$

Ce-141

\begin{tabular}{|c|c|}
\hline $1.525 \mathrm{E}+03$ & 4.0 \\
\hline $2763 E+03$ & 18.0 \\
\hline 4. $26.2 E+04$ & 4. 1 \\
\hline 1. $145 E+02$ & 20.7 \\
\hline A. $121 E+O 3$ & 24.0 \\
\hline $6.063 E+03$ & 15.8 \\
\hline $1.592 E+03$ & 10.0 \\
\hline $3.600 E+01$ & B. 7 \\
\hline 1. $132 E+O 2$ & 3. 7 \\
\hline 1. $40 B E+02$ & 100.8 \\
\hline $1.578 E+02$ & 100.3 \\
\hline 1. $.30 E-01$ & 27.2 \\
\hline $9.932 E_{+}+00$ & 93.3 \\
\hline 7. $463 E+01$ & 100.1 \\
\hline $6.714 \mathrm{E}+00$ & 7.4 \\
\hline $3.893 \mathrm{E}+00$ & 2.8 \\
\hline 1. $119 E+01$ & 80.3 \\
\hline $5.981 E+00$ & 9.5 \\
\hline $5.321 E+00$ & 12.0 \\
\hline $6.890 E+00$ & 90.5 \\
\hline $6.467 \mathrm{E}-01$ & 16.7 \\
\hline $6.394 E+01$ & 3.6 \\
\hline $6.673 E+00$ & 4.7 \\
\hline 4. 490 P POO & 4.8 \\
\hline $7.501 \mathrm{E}-01$ & 5.8 \\
\hline $5.592 E+00$ & 12.4 \\
\hline $9.999 E-03$ & 100.2 \\
\hline $5.049 E+00$ & 3.9 \\
\hline 2. $005 E+01$ & 8.4 \\
\hline $1.238 E+04$ & 4.0 \\
\hline 4. $854 E+O O$ & 5.4 \\
\hline $7.667 E+01$ & 13.0 \\
\hline $3.509 E+00$ & 101.4 \\
\hline $1.996 E+01$ & 18.5 \\
\hline $1.395 E+02$ & 71.6 \\
\hline $1.619 E+\infty 0$ & 65.2 \\
\hline $4.814 \mathrm{E}-01$ & 80.2 \\
\hline $3.051 E+00$ & 8.3 \\
\hline $1.573 E+03$ & 3.0 \\
\hline 1. $146 E+02$ & 3.8 \\
\hline $5.097 E+01$ & 16.9 \\
\hline $1.171 E+00$ & 8.0 \\
\hline $9.907 E-01$ & 13.4 \\
\hline $4 B 15 E+O 0$ & 4.6 \\
\hline $6.701 E-01$ & 5.5 \\
\hline $2.610 E+00$ & 5.7 \\
\hline $5.668 \mathrm{E}-01$ & 17.1 \\
\hline 5. $110 E-01$ & 100.8 \\
\hline $3.863 E+00$ & \\
\hline
\end{tabular}

Nd- 147

\begin{tabular}{|c|c|}
\hline $1.006 E+0.4$ & \\
\hline $3.777 \mathrm{E}+03$ & 222 \\
\hline 8.517E+0. & 3 . \\
\hline $5.027 E+02$ & \\
\hline 2. $566 \mathrm{E}+04$ & \\
\hline $5.5 B\{E+O 3$ & \\
\hline 1. $378 \mathrm{BE}+03$ & 14. \\
\hline $2.31 \mathrm{GE}+01$ & 14. \\
\hline $3.229 E+02$ & 3 \\
\hline 2. $351 E+02$ & 100.8 \\
\hline 2. $299 E+02$ & 100.3 \\
\hline 1. $402 E-01$ & 55.7 \\
\hline $1.493 E+01$ & 93.1 \\
\hline 2. $100 E 102$ & 17.3 \\
\hline $5.882 E+00$ & \\
\hline$B .87 B E+O O$ & \\
\hline $2.252 E+01$ & 27.2 \\
\hline $4.418 E+00$ & 12.2 \\
\hline $2.009 E+00$ & 47.8 \\
\hline $4.603 E-01$ & 23.6 \\
\hline 1. $154 \mathrm{E}+00$ & 15. 1 \\
\hline 4. $102 E+01$ & \\
\hline $5.721 E+00$ & \\
\hline 4. OOAE +OO & 6. \\
\hline $5.840 E-01$ & 10.9 \\
\hline $3.073 E+00$ & 54.8 \\
\hline $1.636 E-02$ & 100.2 \\
\hline 4. $51 \mathrm{BE}+O O$ & 3.9 \\
\hline $1.217 \mathrm{E}+01$ & 9.5 \\
\hline $1.417 E+O A$ & 3.9 \\
\hline $2.934 E+00$ & 5.9 \\
\hline 1. $166 E+02$ & 10.6 \\
\hline 3. $260 E+O O$ & 93.9 \\
\hline $1.970 E+02$ & 5.3 \\
\hline 1. $402 \mathrm{E}+\mathrm{O} 2$ & 22.9 \\
\hline $1.691 E+\infty 0$ & 65.2 \\
\hline $9.574 E-01$ & 24.1 \\
\hline $9,168 E+00$ & 5.2 \\
\hline $1.854 E+02$ & 13.0 \\
\hline $8.985 E+01$ & 3.9 \\
\hline $3.290 E+01$ & 18.5 \\
\hline 5. $108 \mathrm{E}-01$ & 11.2 \\
\hline $1.115 E+00$ & 12.9 \\
\hline $4.668 E+O O$ & 5.1 \\
\hline $6.597 E-01$ & 5.2 \\
\hline 5. $559 E+00$ & 3.9 \\
\hline 2. $467 E+00$ & 8.4 \\
\hline 5. $766 E-01$ & 100.8 \\
\hline $2.944 E+O:$ & 3. \\
\hline
\end{tabular}

Eu- 152
Tb- 160

\begin{tabular}{|c|c|}
\hline $.560 E+03$ & 3.7 \\
\hline $1.4906+03$ & 93. 1 \\
\hline $3.818 E+04$ & 4.3 \\
\hline 5. $388 E+01$ & 74.0 \\
\hline 3. $165 E+03$ & 97.0 \\
\hline 1. $40+E+O S$ & \\
\hline $2.251 E \cdot 03$ & 8.5 \\
\hline 4. $539[+01$ & 8. \\
\hline 3. $708 E+O 2$ & 3.5 \\
\hline $1.894 E+02$ & \\
\hline $2.345 E+02$ & 100.3 \\
\hline ?. 1515 O1 & 22.6 \\
\hline 1. $552 E+01$ & 93.2 \\
\hline $2895 E+02$ & 11.6 \\
\hline 3. $528 \mathrm{gE}+\mathrm{OO}$ & \\
\hline A. $+33 E+00$ & 2.8 \\
\hline $9 \cdot .40 O E+O O$ & 79.6 \\
\hline A. $631 E+00$ & 10). 6 \\
\hline 6. $147 \mathrm{E}+00$ & 10.5 \\
\hline $5.159 E+O O$ & 90.6 \\
\hline $7.549 E-01$ & 110 \\
\hline $2.097 E+01$ & 4.0 \\
\hline 3. $227 E+00$ & 5.1 \\
\hline 2. $289 \mathrm{c}+00$ & 6.8 \\
\hline 3.832E-O1 & B. \\
\hline 2. $123 E+00$ & 21.5 \\
\hline 7. $660 E-03$ & 100.2 \\
\hline $5.953 E+00$ & 3. \\
\hline 1. $950 E+01$ & 8. \\
\hline $5.597 E+03$ & 4.7 \\
\hline 2. $048 \mathrm{E}+00$ & B. 1 \\
\hline 1. $40 O E+01$ & 53.9 \\
\hline $3.219 E+O O$ & 34.9 \\
\hline $2.497 E+01$ & 20.3 \\
\hline 1. $919 E+O 2$ & 71.6 \\
\hline 2. $176 E+00$ & 65.0 \\
\hline $9.223 \mathrm{E}-01$ & 29.8 \\
\hline $2.350 E+00$ & 11.6 \\
\hline 3. $197 \mathrm{E}+02$ & 16.8 \\
\hline $4.453 E+01$ & 4.5 \\
\hline 1. $803 E+01$ & 72.9 \\
\hline $4.918 E-01$ & 14.1 \\
\hline $6.747 E-01$ & 18.2 \\
\hline $2.469 E+0 O$ & 8.7 \\
\hline 4. $248 \mathrm{E}-01$ & 8 \\
\hline $3.770 E+00$ & 5.3 \\
\hline 1. $360 E+O O$ & 14.2 \\
\hline 8. $583 E-01$ & 100.8 \\
\hline 1. $526 E+01$ & 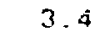 \\
\hline
\end{tabular}

Yb- 169

\begin{tabular}{|c|c|}
\hline & \\
\hline 3. $852 E+03$ & 22.3 \\
\hline B. $836 E+04$ & 3.6 \\
\hline 6. $193 E+02$ & 8.5 \\
\hline $3.020 E+04$ & \\
\hline 7. $A 61 E+03$ & 17.9 \\
\hline $6.975 E+02$ & 96.0 \\
\hline $.089 E+00$ & 100.0 \\
\hline 4. $314 E+O 2$ & \\
\hline $3.465 E+02$ & 100.8 \\
\hline 3. $344 E+O 2$ & 100.3 \\
\hline $2.018 E-01$ & 55.8 \\
\hline 2. $184 E+01$ & 93.1 \\
\hline $1549 E+O 2$ & 100.1 \\
\hline 1. $102 E+01$ & 7.5 \\
\hline 1. $545 \mathrm{E}+\mathrm{O} 1$ & 2.8 \\
\hline $2.127 E+O 1$ & 78.6 \\
\hline $2.041 E+O 1$ & B. 0 \\
\hline $1.987 E+O O$ & 48.0 \\
\hline $3.662 E+00$ & 8.9 \\
\hline $2.598 E+\infty 0$ & B. 1 \\
\hline 3. $339 E+01$ & 3.8 \\
\hline $1 . \therefore 8 E+01$ & 4.9 \\
\hline $7.350 E+0 C$ & 4.7 \\
\hline 1. $121 \mathrm{E}+00$ & 6.2 \\
\hline $2.799 \mathrm{E}+\mathrm{OC}$ & 24.5 \\
\hline 1. $597 \mathrm{E}-02$ & 100.2 \\
\hline 2. $378 E+00$ & 4.0 \\
\hline 6. $390 E+00$ & 22.7 \\
\hline 1. $062 E+04$ & 4. 1 \\
\hline 2. $409 E+O O$ & 7.2 \\
\hline 1. $720 E+02$ & 10.7 \\
\hline 1. $459 E+00$ & 101.4 \\
\hline $2.600 E+O 2$ & 5.2 \\
\hline $2.024 E+02$ & \\
\hline $1.374 E+O 0$ & 65.8 \\
\hline $1.994 E+00$ & 20.6 \\
\hline $1.051 E+01$ & 5.3 \\
\hline 1. $287 \mathrm{E}+02$ & 14.5 \\
\hline 8.009E +01 & 3.9 \\
\hline $3.999 E+09$ & 19.0 \\
\hline $2.875 E-01$ & 14.9 \\
\hline $1.735 E+O O$ & 9.0 \\
\hline 8. $490 E+00$ & 4.1 \\
\hline 1. $1 \triangle B E+\infty O$ & 4.7 \\
\hline 1. $.408 E+01$ & 3.4 \\
\hline $4.693 E+00$ & 7.2 \\
\hline $747 E-01$ & 100.8 \\
\hline $.533 E+01$ & 3.0 \\
\hline
\end{tabular}

LU- 177

Hf -181

ra- 203

Th-233 


$\begin{array}{lc}\text { FILE IO } & 6101890 \\ \text { SAMPLE } & 145 \\ \text { DUPLICATE } & 103 \\ \text { CLUSTER } & 08 \\ \text { TYPE } & T\end{array}$

$\mathrm{Na}-24$

$\mathrm{Mg}-27$

A) - 28

$\mathrm{C} 1-38$
$\mathrm{~K}-42$

$K=42$

$\mathrm{Ca}-49$
$\mathrm{~T} 1-51$

$v-52$

$M n-56$

Cu- 66

$\mathrm{Sr}-87$

In- 116

$\mathrm{Ba}-139$

Dy-165

u -235

$\mathrm{Ga}-72$

As - 76

$\mathrm{Br}-82$

Mo- 99

Sb-122

La- 140

Sm-153

Yb- 175

LU -177

W -187

Au- 198

Sc- 46

$\mathrm{Cr}-51$

$\mathrm{Fe}-59$

$\mathrm{CO}-60$

Zn- 65

Se- 75

$\mathrm{Rb}-\mathbf{8 6}$
$\mathrm{Zr}-95$

$\mathrm{Ag}-110$

Ag- 110

Cs -134

Ba-131

Ce- 141

Nd- 147

Eu-152

Tb- 160

Yb-169

LU- 177

$\mathrm{Hf}-181$
$\mathrm{Ta}-182$

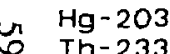

9. $015 \mathrm{E}+03$ 2. $947 \mathrm{E}+03$

9. $310 E+O 4$

5. $740 \mathrm{O}+\mathrm{O} 2$

$2.481 E+04$

6. $114 \mathrm{E}+03$

B. $487 \mathrm{E}+02$

$-6.516 E+0 O$

$-2.507 E+02$

$-2.641 \mathrm{E}+\mathrm{O} 2$

$-2.641 E+02$
$-1.527 E-01$

- 1. $710 E+01$

$-1.072 E+02$

$4.031 E+00$

1. $083 E+01$

$2.033 \mathrm{E}+01$

7. $138 \mathrm{E}+O 0$

$-1.942 E+O O$

4. $933 E+C O$

1. $184 \mathrm{E}+\mathrm{OO}$

2. $806 \mathrm{E}+01$

$3.699 E+O O$

2. $78 \mathrm{OE}+\mathrm{OO}$

3. 905E-0 1

2. $622 \mathrm{E}+00$

1. $527 \mathrm{E}-\mathrm{O} 2$

5. $363 \mathrm{E}+00$

2. $433 \mathrm{E}+00$

5. $669 \mathrm{E}+\mathrm{O} 3$

4. $172 \mathrm{E}-\mathrm{O}$

1. $176 \mathrm{E}+02$

3. $098 E+00$

2. 189E+O2

$7.875 E+01$

$1.077 \mathrm{E}+00$

6. $448 \mathrm{E}+00$

$4.923 E+0$

$6.400 E+01$

2. $257 \mathrm{E}+\mathrm{O}$

4. $838 \mathrm{E}-\mathrm{O}$

5. $933 \mathrm{E}-01$

$3.061 \mathrm{E}+00$

4. 423E-O

5. $137 \mathrm{E}+\mathrm{OO}$

$2.327 \mathrm{E}+0 \mathrm{O}$

$3.327 \mathrm{E}+\mathrm{O}$
3. 1

3.5
3.5

8

9.7

17.2

24.6

3.5

100.8

55.3

93.2

9.2

2. 8

78.8
9.8

47.7

16.0

12.4

4.0

20.3

27.4

100.2

3.9

100.1

4.4
17.2

11.8

96.2

65.1

5.8

22.1

4.0

25. 1

11.5

6.7

6.5

8. 7

100. 8
- . . . . . . . . .

1. $975 E+03$

3. 4

3. $348 E+04$

2. $783 \mathrm{E}+\mathrm{O} 3$

1. $428 \mathrm{E}+04$

72.9

96.9

11.0

$.051 \mathrm{E}+03 \quad 8.9$

$\begin{array}{ll}3.918 E+O 1 & 8.3 \\ 2.706 E+02 & 3.5\end{array}$

$-1.754 \mathrm{E}+02 \quad 100.8$

$-2.001 E+02 \quad 100.3$

1.14 IE-O1 27.5

$\begin{array}{rl}.291 E+01 & 93.2\end{array}$

3. $685 \mathrm{E}+00 \quad 8.2$

3. $691 \mathrm{E}+00$

$-9.692 E+00$

3. 12.1

$-4.910 E+00 \quad 90.8$

$6.235 \mathrm{E}-01 \quad 13.2$

$2.017 \mathrm{E}+01 \quad 4.0$

3. $393 E+00 \quad 4.8$

1.921E+OO 6.9

1. $589 E+00 \quad 25.7$

$-7.495 E-03$

$6.005 E+00 \quad 3.9$

1.828E+01 8.

. 4.4

3. $453 E+O O$
$3.577 E+O 1$

$-2.682 \mathrm{E}+0 \mathrm{O} \quad 67.5$

$2.265 \mathrm{E}+01 \quad 15.7$

71.6

$\begin{array}{ll}-2.053 E+00 & 65.2 \\ -6.384 E-01 & 81.8\end{array}$

3. $351 \mathrm{E}+00$ ก.3

$3.408 E+02 \quad 13.5$

$4.826 \mathrm{E}+01 \quad 4.4$

$-1.614 \mathrm{E}+01 \quad 73.2$

5. $218 E-01$

$.415 \mathrm{E}+00 \quad 8.9$

. 582E-01 8.6

3. $566 \mathrm{E}+0 \mathrm{OO} \quad 5.2$

$1.100 E+00 \quad 14.0$

$\begin{array}{rr}-8.117 E-01 & 100.8 \\ 1.201 E+01 & 3.5\end{array}$
$2.718 E+03$

1. $928 \mathrm{E}+03$

4. $556 \mathrm{E}+04$

8. $280 E+O 1$

-3. $361 \mathrm{E}+03$

1. $507 \mathrm{E}+\mathrm{O} 4$

2. $105 \mathrm{E}+03$

2. $977 E+01$

A. $113 E+02$

$-2.000 E+02$

-2. $615 E+02$

1. $852 \mathrm{E}-01$

2. $260 \mathrm{OE}+\mathrm{O} 2$

3. $041 \mathrm{E}+\mathrm{OO}$

4. $742 E+O O$

1. $100 E+01$

4. $421 E+O O$

3. $142 \mathrm{E}+\mathrm{OO}$

1. $944 \mathrm{E}+0 O$

6. $880 E-O 1$

3. $776 \mathrm{E}+\mathrm{OO}$

$3 E+O O$

4. $233 E-O 1$

4. $006 E+00$

8. $735 \mathrm{E}-03$

5. $606 \mathrm{E}+0 \mathrm{O}$

9. $713 E+O O$

9. $189 \mathrm{E}+03$

$.964 E+O O$

3. $619 E+01$

$-1.011 E+00$

1. $997 E+01$

$-1.604 \mathrm{E}+00$

$-4.401 E-01$

2. $183 E+00$

$-1.927 E+02$

9. $267 \mathrm{E}+0 \mathrm{O}$

4. $969 E-O$

4. $006 E-01$

2. $369 \mathrm{E}+00$

$4.471 \mathrm{E}+\mathrm{OO}$

1. $016 \mathrm{E}+\mathrm{OO}$

$-5.987 E-01$

1. $379 E+O 1$
3.6
33.5

30.5

97.1

11.3
10.9

10.4

3.5
100.8

00.3

23.5

93. 2

11.6

2. 8

79.6

10. 2

15.1

16.6
13.6

4.0

4. 9

6. 6

14.3

100.2

3.9

10. 3

4.

7.2
19.8

94.7

19. 2

94.1

64.9

84. 9

10.0
41.4

41.4
4.3
76.2

11.9

21. 4

7.0

4. 4

12.5
100.8

100.8
3.3

3. $288 E+03$

1. $280 E+03$

$4.026 \mathrm{E}+\mathrm{O} 4$

$884 \mathrm{E}+01$

1. $461 E+04$

1. $550 E+03$

2. $23 B E+01$

4. $31 \mathrm{BE}+02$ 


$\begin{array}{lc}\text { FILE ID } & 6101930 \\ \text { SAMPLE } & 149 \\ \text { DUPLICATE } & 095 \\ \text { CLUSTER } & 08 \\ \text { TYPE } & 1\end{array}$

6101940
150
082
10
1

6101950
151
094

08

05
6101951

152

156

L

\section{ELEMENT}

\begin{tabular}{|c|c|c|}
\hline $\mathrm{Na}-24$ & G. $693 E+03$ & \\
\hline $\mathrm{Mg}-27$ & $1273 E+04$ & 8.6 \\
\hline$A \overrightarrow{1}-28$ & $9.773 E+04$ & 3.4 \\
\hline$C 1-38$ & $-7.071 E+01$ & 72.4 \\
\hline$k-42$ & $9.835 E+03$ & 14.8 \\
\hline $\mathrm{Ca}-49$ & 1. $191 E+O 4$ & 12.7 \\
\hline$T_{1}-51$ & $2.579 E+03$ & 7.9 \\
\hline$v-52$ & $3.283 E+01$ & 9.7 \\
\hline$M n-56$ & 2. $469 \mathrm{E}+\mathrm{O} 2$ & 3.0 \\
\hline $\mathrm{CU}-\mathrm{GG}$ & $2.341 E+02$ & 100.8 \\
\hline $5 r-87$ & $-2.264 E+02$ & 100.3 \\
\hline In-116 & $1.523 E-01$ & 25.4 \\
\hline I -128 & $-1.462 E+01$ & 93.2 \\
\hline Ba- 139 & 3. $548 E+02$ & 11.0 \\
\hline$D y-165$ & $3.022 \mathrm{E}+00$ & 9.1 \\
\hline$u-235$ & $1.123 E+04$ & 2.8 \\
\hline Ga- 72 & $1.999 E+01$ & 24.4 \\
\hline As - 76 & 4. $04 O E+O O$ & 11.7 \\
\hline$B r-A 2$ & $-1.217 E+\infty O$ & 47.8 \\
\hline Mo- 99 & $-6.364 E+\infty O$ & 90.8 \\
\hline $56-122$ & 1. $379 E+00$ & 10.2 \\
\hline$L \Delta-140$ & 2. $149 E+01$ & 4.0 \\
\hline $5 m-153$ & $2.637 \mathrm{E}+00$ & 7.3 \\
\hline$Y b-175$ & $1.424 E+00$ & 13.4 \\
\hline$L \mathrm{Lu}-177$ & $1.995 E-01$ & 44.1 \\
\hline$w-187$ & $-1.643 E+00$ & 48.9 \\
\hline$A L-198$ & $-9.860 \mathrm{E}-03$ & 100.2 \\
\hline Sc- 46 & $7.445 E+00$ & 3.9 \\
\hline $\mathrm{Cr}-51$ & $1.706 E+01$ & 9.4 \\
\hline Fe- 59 & $1.961 E+04$ & 3.9 \\
\hline $\mathrm{Co}-60$ & $3.044 E+\infty 0$ & 6.5 \\
\hline $2 n-65$ & $5.862 \mathrm{E}+01$ & 16.8 \\
\hline $5 e-75$ & $2.518 E+00$ & 33.9 \\
\hline Rb- $\mathbf{9 6}$ & $5.725 E+01$ & 12.0 \\
\hline $2 r-95$ & 1. $369 \mathrm{E}+02$ & 28.2 \\
\hline $\mathrm{Ag}-110$ & $-2.485 E+00$ & 65.3 \\
\hline $5 b-124$ & $1.548 E+00$ & 29.8 \\
\hline$C s-134$ & G. $7 G 7 E+\infty O$ & 6.4 \\
\hline $8 a-131$ & $3.015 E+02$ & 16.8 \\
\hline$C e-141$ & 4. $886 E+01$ & 4.2 \\
\hline Nd- 147 & $-2.155 E+01$ & 72.2 \\
\hline Eu- 152 & $4.560 E-01$ & 1.4 .2 \\
\hline$T D-160$ & $1.261 E-01$ & 25.5 \\
\hline$Y O-169$ & 1. $939 E+00$ & 14.8 \\
\hline$L u-177$ & 3. $107 \mathrm{E}-01$ & 11.8 \\
\hline$H f-181$ & $6.016 E+00$ & 4.4 \\
\hline$T a-182$ & $1.773 E+00$ & 11.2 \\
\hline $\operatorname{Hg}-203$ & $-1.002 E+00$ & 100.8 \\
\hline & $3.954 E+01$ & \\
\hline
\end{tabular}

\begin{tabular}{|c|c|}
\hline $2.6 .98 E+03$ & 3.5 \\
\hline $2.774 E+03$ & 18.6 \\
\hline $3.330 E+0$. & \\
\hline $4.227 E+01$ & 73.7 \\
\hline $2289 E+03$ & 97.1 \\
\hline $1.424 E+04$ & 12.3 \\
\hline $1.67 \mathrm{AE}+03$ & 10.8 \\
\hline $4.794 E+0$ & 7.3 \\
\hline $2.797 E+02$ & 3.5 \\
\hline $1.472 E+02$ & 100.8 \\
\hline $1.79 A E+02$ & 100.3 \\
\hline $1.298 E-01$ & 22.4 \\
\hline $178 E+O 1$ & 93.1 \\
\hline $2102 \mathrm{E}+02$ & 13.9 \\
\hline $5.329 E+00$ & 7.4 \\
\hline $2.245 E+00$ & 2.8 \\
\hline $1.353 E+01$ & 79.4 \\
\hline $2.76 G E+0 O$ & 13.1 \\
\hline $5.988 E+00$ & 10.4 \\
\hline 8. $142 E+O O$ & 894 \\
\hline $4558 E-01$ & 21.1 \\
\hline $2.050 E+01$ & 4.1 \\
\hline 3. $475 E+O C$ & 4.7 \\
\hline $4.907 E+00$ & 5.0 \\
\hline 8 $834 E-01$ & 5.4 \\
\hline 2. $396 E+00$ & 23.7 \\
\hline $1.011 E-02$ & 100.2 \\
\hline 7. $253 \mathrm{E}+00$ & 3.8 \\
\hline 2. $268 E+01$ & 6.7 \\
\hline $1.077 \mathrm{E}+0.4$ & 4.0 \\
\hline $7.576 E+00$ & 4.7 \\
\hline $7.107 \varepsilon+01$ & 12.8 \\
\hline 3. $311 \varepsilon+O O$ & 246 \\
\hline 3. $262 \mathrm{E}+\mathrm{O} 1$ & 12.7 \\
\hline $1.649 E+02$ & 71.5 \\
\hline $1.911 E+00$ & 65.1 \\
\hline $5.593 \mathrm{E}-01$ & 79.4 \\
\hline 3. $352 E+00$ & 7.5 \\
\hline $1.976 \mathrm{E}+02$ & 19.2 \\
\hline $4.68 \mathrm{AE}+0$ & 3.9 \\
\hline $9.360 E+00$ & 77.9 \\
\hline $6.875 E-01$ & 9.3 \\
\hline 5. $454 E-01$ & 18.1 \\
\hline $6.321 E+O 0$ & 3.7 \\
\hline $9.376 \mathrm{E}-01$ & 4. 6 \\
\hline 3.2 $18 E+00$ & 4.8 \\
\hline $5.970 E-01$ & 16.4 \\
\hline $6.721 E-01$ & 100.8 \\
\hline $7.917 E+00$ & 3.5 \\
\hline
\end{tabular}

\begin{tabular}{|c|c|c|}
\hline & $912 E+03$ & 3.5 \\
\hline & $034 E+03$ & 24.6 \\
\hline & $168 E+O A$ & 4.3 \\
\hline & $165 E+01$ & \\
\hline & $258 \varepsilon+03$ & 27.2 \\
\hline & $3.46 E+04$ & 11.6 \\
\hline & $102 E+03$ & B. 7 \\
\hline & $148 E+01$ & 7.5 \\
\hline & $826 E+02$ & 3.5 \\
\hline & $675 E+02$ & 100.8 \\
\hline & $988 E+02$ & 100.3 \\
\hline & $729 E-01$ & 17.3 \\
\hline & $299 E+01$ & 93.2 \\
\hline & $181 E+02$ & 15.2 \\
\hline & $123 E+00$ & 7.2 \\
\hline & $141 E+00$ & 2.8 \\
\hline & $719 E+O O$ & 79.7 \\
\hline & $186 E+00$ & 11.2 \\
\hline & $40.3 E+00$ & 10.5 \\
\hline & $927 E+00$ & 90.6 \\
\hline & $988 E-01$ & 14.6 \\
\hline & $982 E+01$ & 4.0 \\
\hline . & $757 \mathrm{E}+00$ & \\
\hline & $804 E+00$ & 4.7 \\
\hline & 059E-01 & A. 9 \\
\hline & $874 E+00$ & 19.8 \\
\hline & $472 E-03$ & 100.2 \\
\hline 7. & $40 O E+O O$ & 3.9 \\
\hline & $97 O E+01$ & 8.4 \\
\hline$i$. & $.097 E+04$ & A. 1 \\
\hline & $636 E+00$ & 4.9 \\
\hline 4. & $56 \mathrm{BE}+01$ & 18.6 \\
\hline 3. & $609 E+00$ & 24.5 \\
\hline 3. & $228 E+01$ & 19.1 \\
\hline 2. & $.057 E+02$ & 71.5 \\
\hline 2. & $29 A E+00$ & 65.4 \\
\hline & $405 E-0$ & 81.9 \\
\hline 3. & $715 E+00$ & 8.6 \\
\hline & $701 E+O 2$ & 24.8 \\
\hline 4. & $755 E+01$ & 4. 6 \\
\hline & $805 E+01$ & 73.3 \\
\hline & $877 \mathrm{E}-01$ & 10.6 \\
\hline & $789 \mathrm{E}-01$ & 18.6 \\
\hline & $.016 E+00$ & 4. 3 \\
\hline & $.081 E-01$ & 5.3 \\
\hline & $.965 E+O O$ & 5.6 \\
\hline & $.035 E-01$ & 41.2 \\
\hline & $.729 E-01$ & 100.8 \\
\hline & & 3 \\
\hline
\end{tabular}

\begin{tabular}{|c|c|}
\hline $5.059 E+02$ & \\
\hline 1. $183 E+03$ & 94.7 \\
\hline $9.505 E+03$ & 7.1 \\
\hline $5.492 E+01$ & \\
\hline $2.650 E+03$ & 97.4 \\
\hline $1.957 E+04$ & \\
\hline$-4.798 E+02$ & 94.7 \\
\hline $1.932 E+01$ & 14.7 \\
\hline $3.801 E+02$ & 3.5 \\
\hline $1.520 E+02$ & 100.8 \\
\hline $2.347 \mathrm{E}+\mathrm{O} 2$ & 100.3 \\
\hline 1.2 AOE - O 1 & 27.9 \\
\hline$-1.518 E+01$ & 93.4 \\
\hline$-1.037 E+02$ & 100.1 \\
\hline$-9.077 \mathrm{E}-01$ & 67.6 \\
\hline $1.2,1 E+\infty 0$ & 2.8 \\
\hline$-6.603 E+00$ & \\
\hline 2. $608 E+O O$ & 10.8 \\
\hline $7.288 E+00$ & 8.9 \\
\hline$-3.525 E+00$ & 90.5 \\
\hline $3.336 E-01$ & 14.4 \\
\hline $5.830 E+O O$ & 5.3 \\
\hline 1. $142 E+O O$ & \\
\hline $1.030 E+00$ & 8. 3 \\
\hline 1. $4 A 4 E-O 1$ & 11.2 \\
\hline$-8.186 E-01$ & 82.7 \\
\hline$-5.144 E-03$ & 100.2 \\
\hline $2.676 \mathrm{E}+00$ & 3.9 \\
\hline 4. $4 \mathrm{~B} 3 \mathrm{BE}+0 \mathrm{O}$ & 15.0 \\
\hline $3.798 E+03$ & 4.7 \\
\hline $1.522 E+O O$ & 7.8 \\
\hline $1.677 E+01$ & 31.3 \\
\hline $2.680 E+00$ & 22.8 \\
\hline$-6.272 E+00$ & 100.0 \\
\hline$-9.7 O B E+O 1$ & 71.6 \\
\hline$-1.122 E+0 O$ & 65.2 \\
\hline$-3.273 E-01$ & 64.3 \\
\hline$-2.900 E-01$ & 58.3 \\
\hline$-1.016 E+02$ & 54.0 \\
\hline $1.266 \varepsilon+01$ & 5.2 \\
\hline$-\mathfrak{6} .2 B B E+O O$ & 76.7 \\
\hline $1.765 E-01$ & 17.5 \\
\hline$-1.089 E-01$ & 100.2 \\
\hline 1. $355 E+\infty 0$ & 7.9 \\
\hline $1.77 \mathrm{BE}-01$ & 10.4 \\
\hline $1.348 E+O O$ & 7.5 \\
\hline $3.946 E-01$ & 25.9 \\
\hline$-4.301 E-01$ & 100.8 \\
\hline & \\
\hline
\end{tabular}




$\begin{array}{lc}\text { FILE ID } & 6101960 \\ \text { SAMPLE } & 153 \\ \text { DUPLICATE } & 075 \\ \text { CLUSTER } & 10 \\ \text { TYPE } & \text { L }\end{array}$

1. $861 E+03$

$-1.527 \mathrm{E}+03$

1. $752 \mathrm{E}+\mathrm{O} 4$

$-5.710 E+01$

- $3.369 E+03$

. $584 \mathrm{E}+04$

. $374 \mathrm{E}+03$

1. $930 E+01$

4. $919 E+02$

$-2.047 \mathrm{E}+02$

-2. $920 E+O 2$

. $620 E-01$

- 1. $923 E+01$

2. $746 \mathrm{E}+\mathrm{OO}$

$1.671 E+O O$

$-1.068 E+01$

2. $713 E+O O$

$4.412 \mathrm{E}+\mathrm{OO}$

. $063 \mathrm{E}+00$

$.804 E-O 1$
$.538 E+0 O$

1. $.985 E+00$

3. $05 B E+00$

5. 625E-O1

$3.391 E+00$

$-7.626 \mathrm{E}-03$

4. $017 E+00$

$.094 E+03$

4. $578 \mathrm{E}+0 \mathrm{O}$

1. $472 \mathrm{E}+01$

3. $491 \mathrm{E}+\mathrm{OO}$

1. $608 \mathrm{BE}+01$

$-1.236 E+02$

$-1.433 E+O 0$

4. $383 \mathrm{E}-\mathrm{O}$

1. $165 E+00$

1. $108 \mathrm{E}+02$

8. $231 E+O O$

3. $713 \mathrm{E}-\mathrm{O}$

3. $178 \mathrm{E}-01$

3. $193 \mathrm{E}+00$

4. $975 \mathrm{E}-\mathrm{O} 1$

3. $303 \mathrm{E}-01$

$-5.365 E-O 1$

$4.978 \mathrm{E}+00$
4. 0

94.

75.6

97.4

11.9
18.2

3.5

3.5

100.8

00. 3

23.2

00.1

13.5

79.8

12.3

12.3

90.7

14.2

4.8

5.5

5

15.4

100.2

3.9

9.9

5.7

44.7

22.8
71.6

64.9

82.8

14.5

27.9

4.7
76.4

16.0

20.6

5.2

41.4

100. 8
$6.044 \mathrm{E}+03$

7. $424 \mathrm{E}+03$

1. $191 E+05$

$-5.939 E+01$

1. $170 E+04$

9. $650 E+03$

3. $862 \mathrm{E}+\mathrm{O} 3$

$4.888 E+01$

1. $960 \mathrm{E}+02$

$-2.073 E+02$

$-1.929 E+O 2$

2. 17OE-O1

$-1.265 E+01$

6. $480 E+O O$

2. $087 E+01$

2. $159 \mathrm{E}+\mathrm{OO}$

$-1.842 E+O O$

$-1.162 \mathrm{E}+\mathrm{O} 1$

1. $092 E+00$

4. $562 E+01$

2. $574 E+O O$

2. $966 E+00$

3. $738 \mathrm{BE}-01$

$-2.552 E+00$

$-1.463 E-02$

1. $869 E+01$

2. $201 E+04$

1. $979 E+O 2$

4. $665 \mathrm{E}+00$

7. $630 \mathrm{E}+01$

$3.597 E+O 2$

$-1.901 E+00$

1. $275 \mathrm{E}+00$

$.931 E+O O$

$5.300 E+02$

1. $019 E+02$

$6.066 \mathrm{E}+01$

1. $244 \mathrm{E}+\mathrm{OO}$

. $368 \mathrm{E}+00$

3. $304 E+00$

4. $111 \mathrm{E}-01$

1. $173 \mathrm{E}+01$

2. 155E+0O

2. $346 \mathrm{E}+01$

\section{2}

12.1

3.4
72.8

3.4

14.1
6.8

8.

3.6

00.8

100.3
19.7

93.

9. 1

2.8

2.8

79.0

20.0

89.6

13.8

3.8
5.0

8. 5

13.7

54.7

100.2

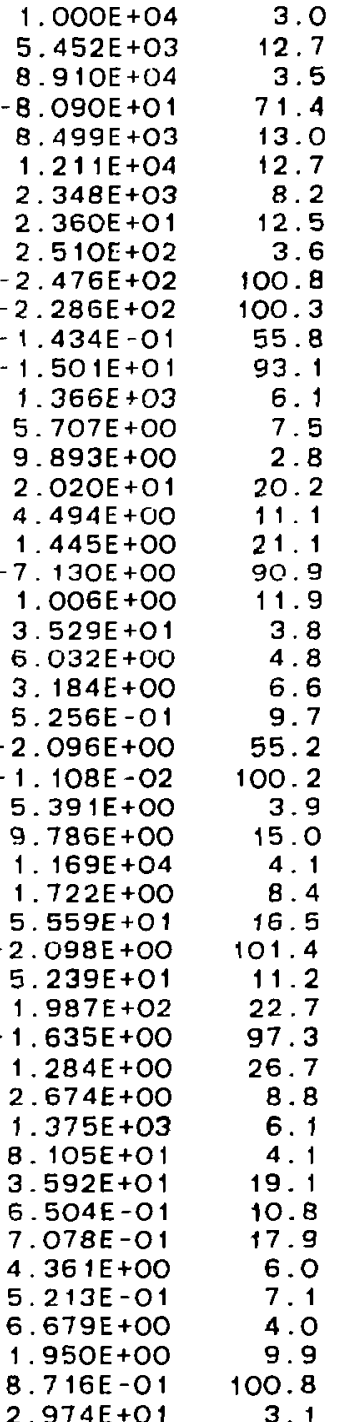

2. $733 \mathrm{E}+03$ $2.219 \mathrm{E}+03$

3. $303 E+O 4$

$-5.070 E+01$

$-2.590 E+03$

1. $516 \mathrm{E}+04$

$2.031 \mathrm{E}+03$

$4.645 E+01$

2. $780 E+02$

1. $726 \mathrm{E}+02$

$2.067 E+02$

$1.755 E-01$

$-1.368 \mathrm{E}+01$

$-9.550 E+01$

4. $643 E+O O$

2. $150 \mathrm{E}+0 \mathrm{O}$

$-1.215 E+01$

3. $707 \mathrm{E}+0 \mathrm{O}$

6. $517 \mathrm{E}+00$

$-5.509 E+O O$

8. $418 E-O 1$

2. $110 E+01$

3. $884 E+O O$

5. $618 E+O O$

1. $016 \mathrm{E}+\mathrm{OO}$

$2.097 E+00$

$-8.344 E-O 3$

7. $289 \mathrm{E}+\mathrm{OO}$

1. $931 E+O 1$

1. $040 E+04$

7. $376 \mathrm{E}+\mathrm{OO}$

6. $017 \mathrm{E}+\mathrm{O} 1$

2. $151 \mathrm{E}+01$

- $1.083 E+02$

$-1.686 \mathrm{E}+00$

$-1.686 E+O D$

3. $579 \mathrm{E}+\mathrm{OO}$

1. $796 \mathrm{E}+\mathrm{O} 2$

4. $375 E+01$

$-8.88 O E+O O$

6. $469 \mathrm{E}-01$

$1.064 E+00$

5. $591 E+O O$

9. $365 E-O 1$

$5.569 E-O 1$
$-6.197 E-01$

B. OOOE +OO
3.5
27.3

4.2

73.4

11.0

9.4

3. 5

100.8

100.3
20.4

93.2

00. 1

2.8

2.8
79.4

11.1

9.8
90.8

11.7

4. 0

4. 4

4.5

100.2 